hep-th/0207212

FIAN/TD/14/02

July 2002

\title{
Conformal Higher Spin Theory
}

\author{
Arkady Y. Segal ${ }^{\dagger}$ \\ † I.E.Tamm Department of Theoretical Physics, Lebedev Physical Institute, \\ Leninsky prospect 53, 119991, Moscow, Russia \\ e-mail:segal@lpi.ru
}

\begin{abstract}
We construct gauge theory of interacting symmetric traceless tensors of all ranks $s=0,1,2,3, \ldots$ which generalizes Weyl-invariant dilaton gravity to the higher spin case, in any dimension $d>2$. The action is given by the trace of the projector to the subspace with positive eigenvalues of an arbitrary hermitian differential operator $\hat{H}$, and the symmetric tensors emerge after expansion of the latter in power series in derivatives. After decomposition in perturbative series around conformally flat point $\hat{H}=\square$ with Euclidean metric, the action functional describes conformal higher spin theory. Namely, the linear in fluctuation term cancels, while the one quadratic in fluctuation breaks up as a sum of conformal higher spin theories, the latter being free gauge theories of symmetric traceless tensors of rank $s$ with actions of $d-4+2 s$ order in derivatives (in odd dimensions they are boundary terms), for all integer $s$, introduced in $4 d$ case by Fradkin and Tseytlin and studied at the cubic order level by Fradkin and Linetsky. Higher orders in interaction are well-defined. The action appears to be the unique functional invariant w.r.t. general similarity transformations $\hat{H}^{\prime}=e^{\hat{\omega}^{\dagger}} \hat{H} e^{\hat{\omega}}$, the latter invariance plays the role of gauge symmetry group of the model. In the framework of the perturbative decomposition, the hermitian part of $\omega$ gauges away the trace parts of the symmetric tensors parameterizing the fluctuation, while the anti-hermitian one provides standard linearized gauge transformations of conformal higher spin fields. The action can be calculated as a semiclassical series in $\hbar$ which counts the number of spacetime derivatives and thereby exhibits itself as a parameter of low-energy expansion,
\end{abstract}


like $\sqrt{\alpha^{\prime}}$ in string theory, in so doing the classical term is given by the volume of the domain $H(x, p)>0$ (where $H(x, p)$ is the Weyl symbol of $\hat{H}$ ), it does not contain derivatives and is interpreted as a cosmological term. At the same time, further terms of the $\hbar$-expansion are given by integrals of distributions localized on the constraint surface $H(x, p)=0$, and the conformal higher spin- $s$ action arises from the $\hbar^{d-4+2 s}$-correction. Next, full gauge invariance of the model is interpreted as covariance algebra of generalized Klein-Gordon equation $\hat{H} \mid \psi>=0$ for complex scalar field $\psi$, and gives rise to the infinite-dimensional global symmetry identified with the algebra of observables of the quantized point particle. Each global symmetry produces a Noether current constructed out of $\psi$ according to general formula we present in the paper. In the case $\hat{H}=\square$ the algebra of observables is an extension of the conformal algebra decomposed w.r.t. its adjoint action as a direct sum of finite-dimensional representations characterized by traceless rectangular two-row Young tableaux. This infinite-dimensional algebra coincides, in $d=3,4,6$ with conformal higher spin algebras constructed before in terms of even spinor oscillators, which origin is due to twistor reformulation of the dynamics of the massless particle. The construction of the paper may be a starting point for diverse conjectures. First, we discuss the extension of the geometry "quantized point particle + conformal higher spin fields in $d$ dimensions" to the one "tensionless $d-1$ brane + higher spin massless fields in $d+1$ dimensions", where the phase of $\psi$ appear to describe transverse motion of the brane inside $d+1$-bulk. This picture arises in the semiclassical approximation to the quantized particle dynamics, the latter approximation provides $d$-dimensional generalization of $W$-geometry elaborated on by Hull in $d=1,2$ case. Next, we propose a candidate on the role of Higgs-like higher spin compensator able to spontaneously break higher spin symmetries. At last, we make the conjecture that, in even dimensions $d$, the action of conformal higher spin theory equals the logarithmically divergent term of the action of massless higher spin fields on $A d S_{d+1}$ evaluated on the solutions of Dirichlet-like problem, where conformal higher spin fields are boundary values of massless higher spin fields on $A d S_{d+1}$, the latter conjecture conforms with recent proposal (for $d=4$ ) by Tseytlin and provides information on the full higher spin action in $A d S_{d+1}$.

A part of the results of this paper was delivered by the author

at the Third International Sakharov Conference, June 22-29, 2002, Moscow

\section{Contents}

1 Introduction and Statement of the results 
2 Low spin data. Linearized data 15

2.1 Low spin data. Weyl invariant dilaton gravity $\ldots \ldots \ldots \ldots$

2.2 Linearized data . . . . . . . . . . . . . . . . . . . . . . . . . 21

$2.2 .1 \quad$ Free conformal higher spin theories . . . . . . . . . . . . . . 21

$2.2 .2 \quad$ Free higher spin theories and the higher spin problem . . . . . . . . 22

2.2 .3 On AdS/CFT "higher spin massless fields in $A d S_{d+1} /$ conformal higher spin fields in $d$-dimensional Minkowski space"] $\ldots$. . . . . . 24

3 Technical digression. Deformed traceless higher spin theories 25

3.1 Deformed traceless higher spin theories. . . . . . . . . . . . . . . 25

3.2 Dressing and Undressing maps . . . . . . . . . . . . . . . . . . . . . . . . . . . . . 29

3.3 Reconstruction map $\ldots \ldots \ldots \ldots \ldots$

$\begin{array}{lll}4 & \text { Geometry } & 32\end{array}$

4.1 Classical geometry . . . . . . . . . . . . . . . . . . . . . . . 34

4.2 Quantum geometry . . . . . . . . . . . . . . . . . . . . . . . 38

4.3 Semiclassics. Generalized $W$-geometry $\ldots \ldots \ldots$. . . . . . . . . . . 41

4.4 Linearization and the theorem on structure of invariant induced action around a flat vacuum. . . . . . . . . . . . . . . . . . . . 44

5 The Action $\quad 45$

5.1 Formal operator approach $\ldots \ldots \ldots \ldots$. . . . . . . . . . 46

5.2 General class of functionals $\operatorname{Tr} \mathrm{F}\left(\mathrm{H}^{*}\right)$. Semiclassical expansion as a low-energy expansion. . . . . . . . . . . 48

5.3 The Action . . . . . . . . . . . . . . . . . . . . . . . 52

5.4 Classes of hamiltonians. $\mathcal{C}, \mathcal{C}_{p}$ and $\tilde{\mathcal{C}}_{p} \ldots \ldots \ldots \ldots \ldots$

5.5 Actual gauge invariance of the action $\ldots \ldots \ldots \ldots \ldots$

6 Restoration of the low spin data. Low spin ansatz: Weyl-invariant dila$\begin{array}{ll}\text { ton gravity } & 60\end{array}$

6.1 Calculation . . . . . . . . . . . . . . . . . . . 60

6.2 Comparison to Schwinger-Dewitt technique . . . . . . . . . . . . . . 64

6.3 Conformally flat vacuum $\ldots \ldots \ldots \ldots \ldots$

\begin{tabular}{lll}
\hline 7 & Conformally flat vacuum. & 69
\end{tabular}

8 Restoration of the linearized data. Perturbative expansion around the $\begin{array}{ll}\text { conformally flat vacuum } & 71\end{array}$ 
$8.1 \quad$ General perturbative expansion. . . . . . . . . . . . . . . . . . . 71

8.2 Calculation of the quadratic part of the action . . . . . . . . . . 73

$8.3 \quad$ Spin decomposition $\ldots \ldots \ldots \ldots \ldots$

8.4 Calculation of the cubic part of the action $\ldots \ldots \ldots \ldots$

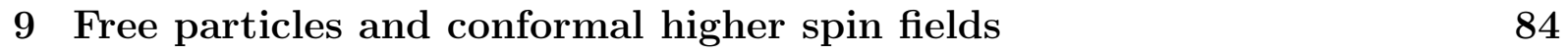

10 Global Symmetries. Conformal algebra and its infinite-dimensional extension, conformal higher spin algebra. Higher spin currents $\quad 86$

10.1 Undressing, Reconstruction, and Conformal invariance. . . . . . . . . . 87

10.2 Algebra of observables of free quantized point particle and all bilinear conserved currents in general background fields. . . . . . . . . . . . . . 89

10.2 .1 Algebra of observables . . . . . . . . . . . . . . . 90

10.2 .2 Noether currents . . . . . . . . . . . . . . . . . . . . 91

10.2 .3 Classical global symmetries $\ldots \ldots \ldots$. . . . . . . . . 96

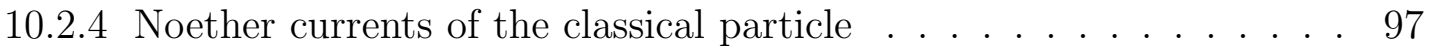

10.2 .5 Noether currents of the Hamilton-Jacobi sigma-model . . . . . . . 97

11 Noether interaction of the scalar particle to gauge fields, and conformal $\begin{array}{ll}\text { higher spin fields } & 98\end{array}$

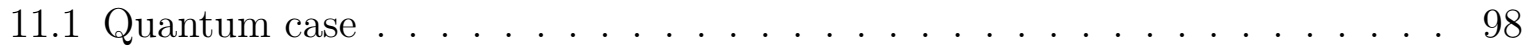

11.2 Classical and semiclassical cast . . . . . . . . . . . . . . . . 100

11.3 On connection with Fradkin-Linetsky approach. . . . . . . . . . . . . . 101

12 On oscillator realizations of conformal higher spin algebras in $d=3,4,6$.

$\begin{array}{ll}\text { Twistors. } & 102\end{array}$

12.1 Quaternion twistors. $D=7, d=6$, higher spin algebra $h s(8 *$. . . . 105

12.2 Complex twistors. $D=5, d=4$, higher spin algebra as even subalgebra of $h s c^{0}(4)=h u_{0}(1,0 \mid 8) . \ldots \ldots \ldots \ldots \ldots \ldots$

12.3 Real twistors. $D=4, d=3$, higher spin algebra as even subalgebra of the Vasiliev's higher spin superalgebra. . . . . . . . . . . . . . 110

$\begin{array}{lr}13 \text { Speculations } & 110\end{array}$

13.1 Higher spin compensator . . . . . . . . . . . . . . . . . . . . . . . 110

13.2 From conformal higher spin fields to Fronsdal higher spin fields. Higher spin fields and tensionless $D-2$-brane. $\ldots \ldots \ldots \ldots \ldots 114$

$\begin{array}{lr}14 \text { Conclusion } & 119\end{array}$ 


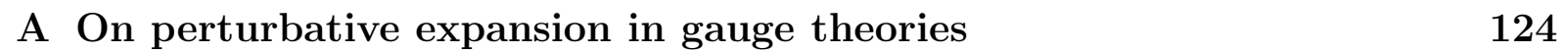

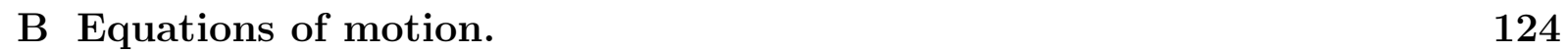

\begin{tabular}{ll}
\hline C Gauge transformations for traceless tensors. & 127
\end{tabular}

D Generating functions for integration over $d$-ball and $d-1$-sphere $\quad 128$

\begin{tabular}{ll}
\hline E Bessel's functions & 130
\end{tabular}

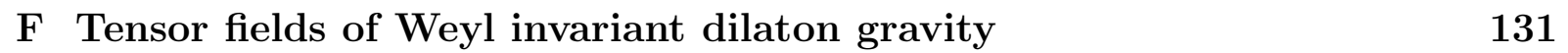

G Free massless scalar and conformal group. 134

H Coordinate representation of an operator with a given Weyl symbol 135

I Weyl spinor formalism in six dimensions 136

\section{Introduction and Statement of the results}

In this paper, we report on the construction of the boson conformal higher spin theory, the gauge theory of infinite number of symmetric traceless tensor fields of all ranks from 0 to $\infty$, in any dimension $d>2$. Each rank- $s$ tensor field enters the spectrum of the theory one time.

The theory described in the paper appears to be the first example, besides the string field theory, of a consistent lagrangian model which includes an infinite number of symmetric tensor fields with arbitrary high rank. In our case, however, one has the theory of symmetric fields only. We construct, proceeding with quite clear principles, the action of the model and show that, after expansion into perturbative series near certain vacuum representing conformally flat space, the quadratic part of the action breaks up as a sum of conformal higher spin theories introduced in $4 d$ case by Fradkin and Tseytlin [四]. These models appear to be important for diverse reasons.

For the first, conformal higher spin theories in $d$ dimensions appear to present a kind of truncation (which may arise in a high energy limit) of higher spin fields theories [2] in $d$ dimensions, the latter describe (at the quadratic level) massless particles of arbitrary spin and could present a new kind of consistent extension of supergravities with infinite number of gauge symmetries [3], [4]. Therefore, the construction of the consistent lagrangian theory 
of conformal higher spin fields (achieved in this paper) is an important step towards solution of the higher spin interaction problem [5] (see Sec.(2.2) below for a little bit more detailed reference to the higher spin problem).

For the second, in view of the $A d S / C F T$ correspondence [6], [7], [8], [9] because conformal higher spin fields in $d$-dimensions arise as boundary values of higher spin fields on $A d S_{d+1}$ [10], [11], the latter statement generalizes standard $A d S / C F T$ consideration of e.g. $A d S_{d+1}$ graviton with boundary values described by $d$-dimensional conformal graviton [6], [7]. Therefore, studying conformal higher spin fields in $d$ dimensions may provide information on $A d S_{d+1}$ higher spin massless fields.

For the third, conformal higher spin fields appear to be inherently related to the point particles. Namely, it will be shown that general coupling of point particle to background fields is parameterized exactly by conformal higher spin fields and, as a byproduct, point particles play a role of a source for conformal higher spin fields. Being combined with the just mentioned $A d S / C F T$ interpretation of conformal higher spin fields this fact explains the matching "massless higher spin fields in $A d S_{d+1} \leftrightarrow$ all bilinear conserved currents of a free massless complex scalar field on the boundary of $A d S_{d+1}$ " [12 which is in core of recent conjectures on duality of a large- $N$ limit $d=4, \mathcal{N}=4$ SYM theory and tensionless IIB superstring on $A d S_{5} \times S^{5}$ with RR flux [8], [9]. As a byproduct, conformal higher spin fields are able to mediate interactions of point-like sources, as we show in this paper.

In the rest of this section we briefly describe our approach, spell main results and describe the structure of the main text, which appears to be rather extensive. The main reason we do not split the paper into smaller ones is that all considered topics are closely connected to each other and follow almost immediately one from another. Here is the very sketchy list of the topics covered: classical and quantum point particle in general background fields (the latter appear to be symmetric traceless tensors of all ranks), gaugeinvariant action for background fields and its low-energy expansion, perturbative decomposition of the action around a conformally flat vacuum and finding that the quadratic action is a sum of conformal higher spin theories with all integer spins, calculation of the cubic action, global symmetries and infinite-dimensional "conformal higher spin algebras" in any dimension, all conserved currents of free complex scalar field for an arbitrary wave operator and Noether interaction, higher spin compensator that can spontaneously break higher spin gauge symmetries, AdS/CFT correspondence and an extension of geometry "point particle + conformal higher spin fields" in $d$ dimensions to "tensionless $d-1$-brane + massless higher spin fields" in $d+1$ dimensions, interpretation of the conformal higher spin theory action in $d$ (even) dimensions as of logarithmically divergent term of the action of higher spin massless fields in $A d S_{d+1}$ evaluated on solutions of Dirichlet like problem. 
The geometrical setup of the theory springs from the interpretation of the infinite number of symmetric tensors 円

$$
\tilde{H}^{m_{1} \ldots m_{s}}\left(x^{k}\right) s=0,1,2,3, \ldots, k=0,1 \ldots, d-1
$$

as coefficients of the Hermitian differential operatorf

$$
\hat{H}=\sum_{s=0}^{\infty}(-i \hbar)^{s} \tilde{H}^{m_{1} \ldots m_{s}}\left(x^{k}\right) \partial_{m_{1}} \ldots \partial_{m_{s}}, \hat{H}^{\dagger}=\hat{H}
$$

which acts in the linear space of complex wave functions $\psi(x)$ and governs the generalized Klein-Gordon equation

$$
\hat{H} \psi(x)=0
$$

Actually, the action of the model is defined as the trace of the projector to the subspace with positive eigenvalues of $\hat{H}$,

$$
\mathcal{A}[\hat{H}]=\operatorname{Tr} \pi_{+}[\hat{H}]
$$

or, what is the same in some cases (e.g. for even $d$ and $\hat{H}$ with positive dilaton), as a time-independent term that appears in the Schwinger-DeWitt-Seeley like asymptotic decomposition of the trace of the evolution operator

$$
\begin{gathered}
\mathcal{A}[\hat{H}]=\alpha_{0} \\
\operatorname{Tr} \exp (-\tau \hat{H})=\sum_{n} \tau^{n} \alpha_{n}, \tau \rightarrow 0^{+} .
\end{gathered}
$$

The action (4) will be shown to be gauge-invariant w.r.t. transformations of the form

$$
\hat{H}^{\prime}=e^{\hat{\omega}^{\dagger}} \hat{H} e^{\hat{\omega}}
$$

where $\hat{\omega}$ is a general operator of the same form (2) as $\hat{H}$,

$$
\hat{\omega}=\sum_{s=0}^{\infty}(-i \hbar)^{s} \omega^{m_{1} \ldots m_{s}}\left(x^{k}\right) \partial_{m_{1}} \ldots \partial_{m_{s}}
$$

but without hermiticity restrictions and with coefficients $\omega^{m_{1} \ldots m_{s}}(x)$ being smooth functions with a compact support in $x$-space. The standard gauge symmetries are parameterized by the low-rank $\omega$ 's. Namely, the real part of $\omega$ is identified with Weyl dilations, while imaginary parts of $\omega, \omega^{m}$ parameterize $U(1)$ transformations and $x$-diffeomorphisms. When the higher rank fields $\tilde{H}^{m_{1} \ldots m_{s}}, s>2$ are set zero, there arises a truncation of the

\footnotetext{
${ }^{1}$ Although we speak about "tensors", actual behavior of $H^{m_{1} \ldots m_{s}}\left(x^{k}\right)$ w.r.t. $x$-diffeomorphisms presents a deformation of standard tensor transformations, see Sec. (4.2).

$2 \hbar$ is a real constant which serves as an expansion parameter counting number of $x$-derivatives.
} 
model to a version of Weyl-invariant dilaton gravity, in particular, in even dimensions the action is given by the well-known expression for the time-independent term of the Schwinger-Dewitt expansion of the trace of the heat kernel [31].

When all higher rank tensors are switched on, the action (4) provides a gauge invariant theory of symmetric tensors (11). The functional (画) is computed by using the well known technique of symbols of operators [51]. Given operator $\hat{H}$ (2) one introduces its Weyl symbol

$$
H(x, p)=\sum_{s=0}^{\infty} H^{m_{1} \ldots m_{s}}\left(x^{k}\right) p_{m_{1}} \ldots p_{m_{s}},
$$

where $H^{m_{1} \ldots m_{s}}\left(x^{k}\right)$ are related to the components of the operator (2) as $\tilde{H}^{m_{1} \ldots m_{s}}\left(x^{k}\right)=$ $H^{m_{1} \ldots m_{s}}\left(x^{k}\right)+o_{s}\left(\hbar \partial H, \hbar^{2} \partial^{2} H, \ldots\right)$, (see App.(代) for the exact formula). Then any expression built from $\hat{H}$ is rewritten in terms of its Weyl symbol $H(x, p)$. Making use of the Weyl symbol formalism the action functional (4) can be computed in a form of semiclassical series like

$$
\operatorname{Tr} \pi_{+}(\hat{H})=(2 \pi \hbar)^{-d} \int d^{d} x\left[\sum_{k=0}^{\infty} \hbar^{2 k} L_{2 k}\left(H, \partial H, \partial^{2} H, \ldots \partial^{2 k} H\right)\right]
$$

where $L_{2 k}\left(H, \partial H, \partial^{2} H, \ldots \partial^{2 k} H\right)$ are expressions built from the components of the tensor fields $H^{m_{1} \ldots m_{s}}$ and their $x$-derivatives up to order $2 k$, with total degree in $x$-derivatives $2 k$. Therefore, semiclassical expansion exhibits itself as a low-energy one, with $\hbar^{2}$ playing the role similar to that $\alpha^{\prime}$ plays in string theory. The zeroth, "classical", term of the $\hbar$-expansion does not contain $x$-derivatives and exhibits itself as a cosmological term. It appears the same term has an interpretation of the volume of the domain

$$
H(x, p) \geq 0
$$

in the phase space of the classical particle in $d$-dimensions, while $\hbar^{2 k}$-terms with $k>0$, have a form of phase space integrals of densities localized on the "constraint surface"

$$
H(x, p)=0
$$

On the other hand, perturbative expansion of the functional (4) around a particular configuration representing the flat space,

$$
\hat{H}=\hbar^{2} \square \Leftrightarrow H=-p^{2},
$$

where $\square$ and $p^{2}$ are constructed with flat Euclidean metric, appears to possess the following properties:

1) term linear in fluctuation cancels for $d>2$, therefore in $d>2$ (12) is a solution of the classical equations of motion of the action (4); 
2) term quadratic in fluctuation breaks up as a sum of conformal higher spin theories with conformal spin $s=0,1,2,3, \ldots$, which were introduced, in $d=4$ case, by Fradkin and Tseytlin [1] and studied by Fradkin and Linetsky [14]; with the actions of the form

$$
\mathcal{A}_{s}\left[\varphi_{s}\right]=\int d^{d} x \varphi^{a(s)} \square^{\frac{d}{2}-2} P_{a(s) b(s)}(\partial) \varphi^{b(s)},
$$

where $\varphi^{a(s)}$ are symmetric traceless tensors made of the fluctuations of $\hat{H}$ around the vacuum (12), while $P_{a(s) b(s)}(\partial)$ is a Poincaré-invariant differential operator of order $2 s$ in derivatives, satisfying set of identities

$$
P_{a(s) b(s)}=P_{b(s) a(s)}, P_{c a(s-2) b(s)}^{c}=0 \quad, \quad P_{a(s-1) c b(s)} \partial^{c}=0 .
$$

3) all higher orders in the fluctuation are well-defined and can be calculated explicitly. Therefore we interpret the functional (i) as the action of conformal higher spin theory. General structure of the vertices is

$$
\mathcal{A} \sim \int d^{d} x \sum_{l=0}^{\infty} e^{l}(\hbar \partial)^{d-2 l+\sum_{i=0}^{l} s_{i}} \varphi_{s_{1}} \ldots \varphi_{s_{l}},
$$

where partial derivatives act in a diverse way on the rank- $s_{1} \ldots s_{l}$ components of the fluctuation. The summation runs only over that subset of indices for which the degree of $\hbar \partial$ is non-negative.

Besides,

4) For any signature of the metric $\eta_{m n}=\operatorname{diag}(d-q, q)$, which enters (12), the theory possesses an infinite-dimensional global symmetry. Namely, the algebra of observables of the quantized particle being defined as a factor algebra of the subalgebra of gauge symmetries that preserves the vacuum (12),

$$
\hat{\omega}^{\dagger} \hat{H}+\hat{H} \omega=0
$$

by the ideal of trivial symmetries of the form

$$
\hat{\omega}_{\text {triv }}=i \hat{\mu} \hat{H}, \hat{\mu}=\hat{\mu}^{\dagger}
$$

is, in the case (12), an infinite-dimensional conformal higher spin algebra chs $(d-q, q)$, which includes the conformal algebra $s o(d-q+1, q+1)$ as a finite-dimensional subalgebra. For $d=3,4,6$ and Minkowski signature, $\operatorname{chs}(d-1,1)$ appear to coincide with boson higher spin algebras introduced before [14], [21], [32] in terms of even spinor oscillators, the latter appear to arise in our construction via twistor reformulation of the massless particle dynamics in terms of Dirac spinor of the conformal algebra $s o(d, 2)$. 
The global symmetry $\operatorname{chs}(d-q, q)$ acts on fluctuations of $\hat{H}$ linearly, hence each order of the perturbative expansion of the action of the theory is invariant w.r.t. infinitedimensional global symmetry $\operatorname{chs}(d-q, q)$.

To each element of the algebra of observables a Noether current is assigned. We find the expression for all Noether currents of the complex scalar field $\psi(x)$ for an arbitrary background wave operator $\hat{H}$ provided its algebra of observables is known. The expression reads

$$
\begin{gathered}
J_{\omega}^{m}=-\frac{1}{2} \sum_{s=0}^{\infty} \sum_{k=0}^{s} \sum_{l=1}^{s-k} \\
\left\{\frac{(-i \hbar)^{s}(-)^{l-1}}{2^{k}} C_{s}^{k} C_{s-k}^{l} \partial_{m_{1}} \ldots \partial_{m_{l-1}}\left[\psi^{*} H_{, m(k)}^{m(k) m(s-k)}(x)(\hat{\omega} \psi)_{, m(s-k-l)}\right]\right\}+\text { c.c. }
\end{gathered}
$$

where $C_{s}^{k}$ are binomial coefficients and $H^{m(s)}(x)$ are coefficients of the decomposition of the Weyl symbol of the operator $\hat{H}$ in power series in momenta. In the case (12) our set of Noether currents coincides, up to a choice of a basis, with the one obtained by the authors of [12] by direct calculation.

The exposure of the main results of the paper has appeared possible due to the extensive use of the notion of a geometry with a source and of its covariance algebra. Let $O$ be a localized object in $d$-dimensions, whose dynamics is parameterized by a set of variables $o$, and let $S_{H}[o]$ be an action that governs the dynamics of $o$, while $H$ in the subscript stands for the set of all possible functionals of $o$ that determine general form of the action. By definition, the algebra $\mathcal{G}$ of covariance transformations consists of all infinitesimal transformations $g$ that act simultaneously on $O$ and on the set of $H$ in such a way that the action is invariant modulo terms which do not affect classical equations of motion,

$$
S_{H+\delta_{g} H}\left[o+\delta_{g} o\right]=S_{H}[o]+\{\ldots\}
$$

where $\{\ldots\}$ stands for terms which do not affect classical equations of motion. Equivalently, the variation of the action due to change of the functionals $H[o]$ is compensated by transferring to the new variables $o^{\prime}(o)$. We say the last formula determines the geometry with the source (or "test object") O, geometric structure (or "background field") H[o], coupling $S_{H}[o]$, and the covariance algebra $\mathcal{G}$.

Given a geometry with a source, one interprets covariance transformations for $H$ as gauge transformations and looks for a gauge-invariant functional of $H$

$$
\mathcal{A}\left[H+\delta_{g} H\right]=\mathcal{A}[H]+\{\ldots\}
$$

where $\{\ldots\}$ stands for terms which do not affect classical equations of motion. Let us call 
any solution of the last equation as invariant induced action 9 .

In the case of conformal higher spin theory, $\mathcal{O}$ will be the quantized point particle, $H=\hat{H}$ be an operator (2) governing evolution of the wave function of the particle by means of the wave equation (3). The coupling is provided by the quadratic action

$$
S_{H}[\psi]=\int d^{d} x \psi^{*}(x) \hat{H} \psi(x)
$$

while the covariance algebra consist of all transformations of the form

$$
\delta \hat{H}=\hat{\omega}^{\dagger} \hat{H}+\hat{H} \hat{\omega}
$$

which act on the wave function by the rule

$$
\delta \psi(x)=-\hat{\omega} \psi(x)
$$

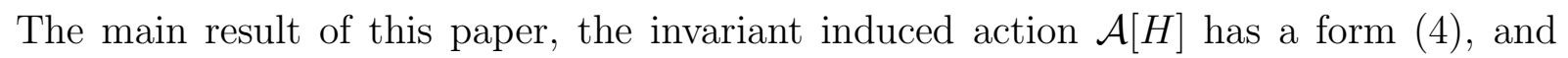
admits the formal path-integral representation

$$
\mathcal{A}[H]=\operatorname{Tr} \theta(H *)=\int \mathcal{D} x \mathcal{D} p \frac{\mathcal{D} \lambda}{\lambda} \delta(\dot{\lambda}) \exp \left\{\frac{i}{\hbar} S_{H}[x, p, \lambda]\right\}
$$

where $S_{H}[x, p, \lambda]$ is Hamiltonian action of the classical particle,

$$
S_{H}[x, p, \lambda]=\int d \tau\left(p_{m} \dot{x}^{m}-\lambda H(x, p)\right),
$$

where $\left(x^{m}(\tau), p_{m}(\tau), \lambda(\tau)\right)$ is the set of particle's phase-space variables which include the Lagrange multiplier $\lambda$ to the first-class constraint $H(x, p)$ being a Weyl symbol of the operator $\hat{H}$. The integration contour for $\lambda$ is not that providing the one-loop effective action $-\frac{1}{2} \operatorname{Tr} \operatorname{Ln} \hat{H}, \lambda \in[\mu, \infty)$ but another one,

$$
\lambda(\tau)=\tau-i \varepsilon ; \tau \in \mathbf{R}, \varepsilon \rightarrow 0^{+}
$$

Thus the conformal higher spin fields exhibit themselves as background fields of the point particle. The united action "invariant induced action+coupling",

$$
\mathcal{A}[\hat{H}, \psi]=\operatorname{Tr} \pi_{+}[\hat{H}]+e \int d^{d} x \psi^{*}(x) \hat{H} \psi(x),
$$

where $e$ is a coupling constant describes a joint dynamics of conformal higher spin fields and quantized point particles. By construction $\mathcal{A}[\hat{H}, \psi]$ is gauge-invariant w.r.t. covariance transformations (22). A vacuum is provided by the configuration

$$
\hat{H}=\hbar^{2} \square, \psi(x)=0,
$$

\footnotetext{
${ }^{3}$ The relation of invariant induced action to the ordinary induced action which looks like $-\frac{1}{2} \operatorname{Tr} \operatorname{Ln} \hat{H}$ will become clear from the below treatment.
} 
which is preserved by the infinite-dimensional global symmetry $\operatorname{chs}(d, 0)$ (16, 17, 12). After analytic continuation to Minkowski space, fluctuations around this vacuum describe interaction of sources built from complex massless field (quantized particle) by means of exchange by conformal higher spin fields, interactions due to exchange by $s=1$ quanta in $d=4$ are familiar Maxwell interactions of charged matter.

This $d$-dimensional system possesses classical conformal invariance and may get $A d S_{d+1}$ dual interpretation. Without doubt, conformal higher spin fields are interpreted as boundary values of massless higher spin fields in $A d S_{d+1}$ (see Sec. (2.2.3)). On the other hand, we show that in a particular low-energy limit the theory of the complex scalar field $\psi(x)$ exhibits itself as a theory of a tensionless $d$-1-brane in $A d S_{d+1}$, where the phase of the wave function is a field that describes position of the $d$-dimensional world volume inside a $d+1$-dimensional bulk. Thus, we conjecture there exists an extension of the geometry "conformal higher spin fields+point particle in $d$ dimensions" to the geometry "massless higher spin fields + tensionless $d-1$-brane in $d+1$ dimensions".

We also show how one could spontaneously break higher spin gauge symmetries of conformal higher spin models by finding a natural candidate on the role of compensator of gauge transformations (22).

Let us describe the structure of the paper along with providing more comments.

Lengthy sections 2 and 3 are preliminary ones.

In Sec. 2, we recall the "initial data" the full model is able to reproduce, namely, the Weyl-invariant dilaton gravity and linearized conformal higher spin theories. Also we describe free Fronsdal theories of higher spin massless fields and show how conformal higher spin theories may arise either as a high-energy truncation of Fronsdal models or as holographic images of the latter via $A d S / C F T$.

In Sec. 3, we provide another preliminary data which we call "deformed conformal higher spin theories", the latter being free Poincaré-invariant models constructed out of infinite set of symmetric tensors, they appear to be related to free conformal higher spin theories by a field redefinition. In our treatment, the latter models will always arise as deformed conformal higher spin theories.

In Sec. 4, we specify geometry that will govern our consideration in the paper and describe in detail the geometry of classical point particle in general background fields, its quantum deformation and semiclassical picture of the quantum geometry, with the coupling provided by a "Hamilton-Jacobi" sigma-model which appear to be a $d$-dimensional generalization of $W$-geometry sigma-model introduced by Hull [13]. In the end of the section, we prove an important theorem that states that once one has constructed an invariant induced action with the vacuum of the form (12) the quadratic expansion of the 
invariant induced action around this vacuum should be a direct sum of conformal higher spin theories with a special value of the deformation parameter.

In Sec. 5, we find a functional of background fields which is invariant w.r.t. covariance transformations, i.e. an invariant induced action. We show it can be expanded in a semiclassical ("low-energy") series (9) and ground its interpretation of the "quantized volume" of a domain in the phase space being bounded by the constraint surface.

In Sec. 6, we show that in the case higher rank fields (with rank $s>2$ ) are zero, the "quantized volume" action reproduces the Weyl-invariant dilaton gravity. We also compare our calculations with the ones that follow from Schwinger-Dewitt method of evaluating traces of integral kernels of evolution operators, find the precise agreement, and formulate the conjecture that, in even dimensions, our action is nothing but the time-independent term that appear in the asymptotic decomposition of the trace of the heat kernel of evolution operator $\exp (i \tau \hat{H})$. This conjecture is in accord with recent reasoning by Tseytlin [11] which is $d=4$, quadratic in fluctuation of $\hat{H}$ version of our one.

In Sec. 0, we show the configuration (12) is a vacuum solution of the equations of motions for the "quantized volume" action. Therefore, by the theorem of Sec. (4), the quadratic term of the expansion of the action around this vacuum should be a sum of conformal higher spin theories.

In Sec. 8, we show by direct calculation that the quadratic term of the perturbative expansion of the "quantized volume" action around the vacuum (12) is nothing but the sum of conformal higher spin theories with conformal spins $s=0,1,2,3, \ldots$ arising in the form of "deformed conformal higher spin models" with a special deformation parameter. The result of this calculation is the main technical result of the paper. Furthermore, we calculate the cubic term of the perturbative expansion of the invariant induced action.

In Sec. 9, we point out once again the conformity of point particles and conformal higher spin fields, and outline the procedure of finding conformal higher spin interactions of point particles.

In Sec. 10, we study algebra of global symmetries of the model. We prove an important lemma that the representation of the conformal algebra on higher spin fields is diagonalized w.r.t. conformal spin and therefore each "conformal higher spin-s" model is conformally invariant indeed. Then we identify algebra of global symmetries of the model with the algebra of observables of a quantized particle. With each global symmetry a Noether current is associated, being a bilinear in the particle's wave function. We find general expression for all Noether currents of a complex scalar field for any wave operator $\hat{H}$. In the case $\hat{H}=-\hbar^{2} \square$ the algebra of observables $\operatorname{chs}(d-q, q)$ contains conformal 
algebra $s o(d-q+1, q+1)$ while the full algebra of observables is decomposed w.r.t. conformal algebra as a sum of finite-dimensional representations described by traceless two-row rectangular Young tables, which is exactly the structure anticipated from the comparison with description of free massless fields on $d+1$-dimensional anti-de Sitter space. We conclude we have constructed boson conformal higher spin algebras in an arbitrary dimension $d$, or, what is the same, the higher spin algebras in dimension $d+1$ (previously, they were known for Minkowski signature and dimensions (if $d>2$ ) $d=3,4,6$ only). We also exhibit the classical limit of the algebra of observables and of the Noether currents.

In Sec. 11, we show that Noether interaction "Noether current-gauge field" of the complex scalar field to one-forms which take values in the algebra of its global symmetries, is a superfluous formulation of the interaction " $\int d^{d} x \psi^{*} \hat{h} \psi$," where $\hat{h}$ is a general fluctuation of the wave operator, expressed via set of one-forms by certain projection, which in fact expresses conformal higher spin fields via one-forms of "gauge description" of conformal higher spin fields introduced by Fradkin and Linetsky in the Vasiliev's-like approach [14].

In Sec. 12, we show that the previously known conformal higher spin algebras in $d=3,4,6$ (=higher spin algebras in $d+1=4,5,7)$ constructed in terms of power series of even spinor oscillators, do coincide with $\operatorname{chs}(d-1,1)$, while the origin of even spinor oscillators is precisely due to the twistor reformulation (available in $d=3,4,6, q=1$ ) of the $d$-dimensional massless particle dynamics in terms of Dirac spinor of the conformal algebra $s o(d, 2)$.

In Sec. 13, we speculate on two diverse subjects, each being related to an extension of the conformal higher spin theory.

In subsec. 13.1, we propose a candidate on the role of higher spin compensator which is a Higgs-like object able to spontaneously break higher spin gauge invariance down to low spin algebra consisting of $x$-diffeomorphisms only, thus we present a first step to the program of breaking higher spin symmetries discussed in [9] .

In subsect. 13.2, we show that the semiclassical approximation to the action of the complex scalar field, having a form of "Hamilton-Jacobi" sigma model of Sec. ॠ, is interpreted as the action of tensionless $d-1$-brane in $d+1$ dimensions, with the phase of the complex scalar field playing the role of the transverse position of the $d$-1-brane world volume inside a $d+1$-bulk. Then we speculate on the existence of $d+1$-dimensional extension of the picture "conformal higher spin fields+quantized point particle in $d$ dimensions" to a system "massless higher spin fields + tensionless $d-1$-brane in $d+1$ dimensions. 
In Conclusion, we discuss some open problems and perspectives.

In Appendix A we collect general facts on perturbative expansion in gauge theories. In App. B, the equations of motions are analyzed in the formal operator approach. App. C contains machinery for handling with infinite collections of symmetric tensors and extracting their traceless parts. App. D provides generating functions for the integrals either over a ball or over $d$-1-spheres in $d$-dimensions. App. E contains necessary formulae on Bessel's functions. App. F deals with fields redefinitions that allow to pass from coefficients of the Weyl symbol of the low-spin wave operator to familiar tensor fields of Weyl-invariant dilaton gravity. App. G contains "two-time" description of massless scalar in $d$ dimensions in terms of a field on the light-cone in $d+2$ dimensions, and calculation of the special operator identity valid for generators of conformal group in this representation. App. H provides the expression for the differential operator with a given Weyl symbol being a power series in momenta. App. I contains Weyl spinor formalism in six dimensions.

On our notation: whenever the indices denoted by the same letter appear their full symmetrization is implied (the full symmetrization is a projector), the symbols like $m(s)$ stand for $m_{1} \ldots m_{s}$. We also use sometimes the notation $A B$ or $(A B)$ for the contraction $A^{k} B_{k}$ of vector $A^{k}$ and covector $B_{k}$.

Let us make a remark on bibliography. The literature on higher spin problem is very extensive, therefore we did not try to present an exhaustive citation of any kind, but rather have drawn most immediate links. The author apologizes for incomplete or missed citations, and would appreciate criticism on this point.

\section{Low spin data. Linearized data}

The theory we construct in the paper possesses "initial data" of two kinds.

One set of initial data is provided by the low-spin truncation, when one sets zero all higher rank tensors (with rank $s>2$ ). In this case the theory reduces to the Weylinvariant dilaton-Maxwell gravity with cosmological term given by the dilaton's potential.

Another set of initial data is provided by free theories of "conformal higher spin fields" on Minkowski space (or its conformal completion), they are gauge theories of symmetric traceless tensors, where for each given rank $s=1,2,3 \ldots$ the theory possesses gauge invariance w.r.t. transformations with a parameter being a symmetric traceless tensor of rank $s-1$. In $d=4$, these free models were described by Fradkin and Tseytlin [1] ("pure spin" models) in the Lorentzian signature case, and were conjectured to be invariant w.r.t. full conformal algebra so $(4,2)$. They possess supersymmetric extensions, studied by Fradkin 
and Linetsky [14. The "pure spin"-s theory is described by an action with $2 s$ derivatives, up to arbitrariness in multiplying the wave operator by an arbitrary function of $\square, f(\square)$. It appears $4 d$ models may be easily generalized to arbitrary dimension and, as we show in the paper, for any $d$ there exists the choice of $f(\square)$ when the "conformal higher spin theories" are conformally invariant indeed.

One of the main messages of the paper is that these two types of data are nothing but different limits of one theory. After constructing the full theory in Sec. (5) we will recover both sets of data by direct calculations. Below we describe them in more detail.

\subsection{Low spin data. Weyl invariant dilaton gravity}

The theory we are going to construct in the paper may be viewed as a higher spin extension of the Weyl-invariant dilaton gravity, the latter is formulated in terms of low spin symmetric tensors $D(x), A_{m}(x), g_{m n}(x)$. The action is required to be invariant w.r.t. gauge transformations

$$
\begin{gathered}
\delta_{\varepsilon} D=-\xi^{m} \partial_{m} \varphi+2 \alpha D \\
\delta_{\varepsilon} A_{m}=-\xi^{k} \partial_{k} A_{m}-A_{k} \partial_{m} \xi^{k}+\partial_{m} \varepsilon \\
\delta_{\varepsilon} g^{m n}=-\xi^{k} \partial_{k} g^{m n}+g^{m k} \partial_{k} \xi^{n}+g^{n k} \partial_{k} \xi^{m}+2 \alpha g^{m n}
\end{gathered}
$$

where $\xi^{m}, \alpha, \varepsilon$ parameterize diffeomorphisms, Weyl dilations and $U(1)$ "gradient" transformations, respectively. The inverse metric $g^{m n}$ and the dilaton $D$ are subject to Weyl dilations with Weyl weights equal to 2 , while $A_{m}$ is Weyl-invariant.

Below we analyze general action invariant w.r.t. these gauge transformations, as in linearized approximation around a flat background as at the full nonlinear level, to exhibit structures that will arise from the action of the conformal higher spin theory.

Let us describe general structure of invariant action. It has a form like f

$$
\begin{gathered}
\mathcal{A}[D, A, g]=w_{d} \int d^{d} x \sqrt{g}\left\{\gamma_{1,0} D^{\frac{d}{2}}+\right. \\
+\gamma_{1,1}\left(D^{\frac{d}{2}-3} g^{m n} D_{m} D_{n}+\frac{4}{(d-1)(d-2)} D^{\frac{d}{2}-1} R\right)+
\end{gathered}
$$

\footnotetext{
${ }^{4}$ We do not pursue complete classification of all possible invariant actions but rather draw general picture of possible structures. This is sufficient for our purposes in the paper.
} 


$$
\begin{gathered}
+\sum_{k=2}^{\infty}\left[\gamma_{1, k} D^{\frac{d}{2}-1-k}\left(\square^{k}+o_{1, k}(R)\right)\right] D+ \\
+g^{m k} g^{n l} F_{m n} \sum_{k=0}^{\infty}\left[\gamma_{2 k} D^{\frac{d}{2}-2-k}\left(\square^{k}+o_{2, k}(R)\right)\right] F_{k l}+ \\
\left.+C_{m n k l} \sum_{k=0}^{\infty}\left[\gamma_{3, k} D^{\frac{d}{2}-2-k}\left(\square^{k}+o_{3, k}(R)\right)\right] C^{m n k l}+\ldots\right\},
\end{gathered}
$$

where $w_{d}, \gamma_{1, k}, \gamma_{2, k}, \gamma_{3, k}, k=0,1,2,3, \ldots$ are constants, $F_{m n}=\partial_{m} A_{n}-\partial_{n} A_{m}$ is the Maxwell field strength, $R$ is scalar curvature, $C_{m n k l}$ is the Weyl tensor, and " $+\ldots$ ". stands for all other Weyl-invariant scalars built from $D, A_{m}, g_{m n}$, in particular, terms of higher degree in curvatures $F_{m n}, C_{m n k l}$. $\square$ is the covariant d'Alambert operator which scales as $\delta \square=2 \alpha \square$ w.r.t. Weyl dilations with constant $\alpha$. Whenever they exist, $\left(\square^{k}+o_{1, k ; 2, k ; 3, k}(R)\right)$ denote Weyl-covariant operators of Weyl weight $2 k$, where $o(R)$ 's stand for appropriate curvature corrections which scale by the same law as $\square^{k}$ w.r.t. Weyl dilations with a constant parameter, like

$$
o_{k} \sim \sum_{l=0}^{k-1}\left(g^{m n}\right)^{k+l}\left(\nabla_{r}\right)^{2 k-2 l}\left(R_{e f g h}\right)^{l},
$$

where upper and lower indices are to be contracted in diverse way. Note that as the scalar curvature $R$ scales by the same law as $\square$ w.r.t. Weyl dilations with constant parameter, $\delta R=2 \alpha r, \alpha=$ const, $o_{k}(R)$ is allowed to contain degrees of $R$ of only $k$-th order. This remark plays an important role below.

The degrees of $D$ and $\square$ in (30) are controlled by the requirement that Lagrangian should have zero Weyl weight, therefore the total degree of $D$ and $\square$ in square brackets in the third row of $(30)$ is equal to $\frac{d}{2}-1$ and in the fourth and fifth row is $\frac{d}{2}-2$. Therefore, in even dimensions, the action (30) contains only integer powers of $D$, while for odd dimensions the action (30) contains only half-integer powers of $D$. It should be noted that the model that arises as a low spin truncation of the full theory of the paper possesses, in even dimensions, only non-negative powers of $D$.

The first term $\gamma_{1,0}(\ldots)$ plays the role of cosmological term. The term $\gamma_{1,1}(\ldots)$ is the standard Weyl-covariant coupling of dilaton to gravity. Indeed, this may be checked either by direct calculation or by making change of variables

$$
D=\varphi^{\frac{4}{d-2}}
$$

that leads to

$$
\begin{gathered}
\int d^{d} x \sqrt{g}\left\{\gamma_{1,0} D^{\frac{d}{2}}+\gamma_{1,1}\left(D^{\frac{d}{2}-3} g^{m n} D_{m} D_{n}+\frac{4}{(d-1)(d-2)} D^{\frac{d}{2}-1} R\right)\right\}= \\
=\int d^{d} x \sqrt{g}\left\{\gamma_{1,0} \varphi^{\frac{2 d}{d-2}}+\gamma_{1,1}\left(\frac{4}{d-2}\right)^{2}\left(g^{m n} \varphi_{m} \varphi_{n}+\frac{d-2}{4(d-1)} R \varphi^{2}\right)\right\}
\end{gathered}
$$


which is the more conventional form of coupling of a conformal scalar to gravity [53]. Note also that the well-known conformally -invariant potential for the dilaton $\varphi$ plays the role of cosmological term.

Let us study expansion of the general action (30) around the following configuration of dynamical variables:

$$
D=\bar{D}=\text { const }, A_{m}=0, g_{m n}=\eta_{m n}
$$

where $\eta_{m n}$ is the Minkowski metric and show that the case $\bar{D}=0$ corresponds, in $d>2$, to a solution of equations of motion of general action (30). Introduce fluctuations $h, h^{m}, h^{m n}$ of $D, A^{m}, g^{m n}$ around the background (34) and study the first, linear in fluctuation, term in the expansion of the action (30). Let us restrict to the case when only non-negative powers of $\square$ enter the action (that will always be the case in our treatment). Then, as the metric is flat, all background curvatures equal zero. As dilaton is constant, $\square^{k} \bar{D}=0$, the same is true for all terms of the sort $\partial_{m} \ldots \partial_{m} \bar{D}$. Thus, the second and the third rows of (30) give zero contributions to the linear action. The only combinations surviving at the linear level come from the first row of (30): from $k=0$ term (the cosmological term)

$$
w_{d} \int d^{d} x\left(\sqrt{g} \bar{D}^{\frac{d}{2}}\right)=w_{d} \int d^{d} x\left(\frac{1}{2} h_{m}^{m} \bar{D}^{\frac{d}{2}}+\frac{d}{2} \bar{D}^{\frac{d}{2}-1} h\right)+O\left(h, h^{m}, h^{m n}\right)
$$

and from $k \neq 0$ terms of the form

$$
w_{d} \int d^{d} x \bar{D}^{\frac{d}{2}-k} \beta_{k} r\left(h_{m n}\right)+O\left(h, h^{m}, h^{m n}\right)
$$

where $\frac{1}{2} h^{m}{ }_{m}$ is the fluctuation of $\sqrt{g}, \beta_{k}$ is the multiple of $R$-linear term in $o_{1 k}$, and $r\left(h_{m n}\right)$ is the fluctuation of the scalar curvature. Recalling the remark after the Eq. (31) one proves that

$$
\beta_{k}=0, k>1
$$

Therefore, the linear part of the action vanishes for $d>2$ provided

$$
\bar{D}=0
$$

This means that in $d>2$, the background configuration

$$
D=0, A_{m}=0, g_{m n}=\eta_{m n}
$$

is a vacuum, i.e. a solution of the classical equations of motion of the action (30).

Useful information may be obtained by studying the linearized gauge transformations. To this end one has to rewrite the gauge transformations (29) in terms of fluctuations 
and keep only terms of zero order in fluctuations. One gets

$$
\begin{gathered}
\delta h^{m n}=-\partial^{m} \xi^{n}-\partial^{n} \xi^{m}-2 \alpha \bar{g}^{m n} \\
\delta h^{m}=\partial^{m} \varepsilon \\
\delta h=0
\end{gathered}
$$

As it is recalled in App. (A), after expansion around the vacuum (39), the quadratic part of the action is invariant w.r.t. linearized gauge transformations.

The scalar fluctuation $h$ is Weyl-inert, and Weyl transformations reside in the rank-2 sector, where they can be used to set $h^{m n}$ traceless,

$$
h^{m}{ }_{m}=0 .
$$

The remaining gauge transformations are

$$
\begin{gathered}
\delta h^{m n}=- \text { Traceless part of }\left(\partial^{m} \xi^{n}+\partial^{n} \xi^{m}\right) \\
\delta h^{m}=\partial^{m} \varepsilon \\
\delta h=0 .
\end{gathered}
$$

This means the quadratic action should be a sum of linearized conformal gravity + Maxwell theory+ scalar theory, where the action of conformal gravity is built from the linearized Weyl tensor and its derivatives. Thus the general structure of the quadratic action in this case should be

$$
\mathcal{A}_{2}\left[h, h^{m}, h^{m n}\right]=\int d^{d} x\left\{h f_{1}(\square) h+F_{m n} f_{2}(\square) F_{m n}+c_{m n k l} f_{3}(\square) c^{m n k l}+\ldots\right\},
$$

where $f_{1,2,3}$ are some functions of $\square, F_{m n}=\partial_{m} h_{n}-\partial_{n} h_{m}, c_{m n k l}$ is the linearized Weyl tensor. This is confirmed by studying the quadratic terms in the perturbative expansion of the action (30) around the vacuum (39). The only nonvanishing terms come from $k=\frac{d}{2}-2$ in the first, second and the third rows of (30), Therefore, around the vacuum (39), the quadratic part of the action (30) reads

$$
\mathcal{A}_{2}\left[h, h^{m}, h^{m n}\right]=\int d^{d} x\left\{\gamma_{1, \frac{d}{2}-2} h \square^{\frac{d}{2}-2} h+\gamma_{2, \frac{d}{2}-2} F_{m n} \square^{\frac{d}{2}-2} F_{m n}+\gamma_{3, \frac{d}{2}-2} c_{m n k l} \square^{\frac{d}{2}-2} c^{m n k l}\right\},
$$

provided the dimension $d$ is even, while in odd dimensions $\mathcal{A}_{2}$ is zero. Nevertheless, the formula (44) may be considered as a definition which is valid in odd dimensions either, if one defines the nonlocal operators $\square^{\frac{k}{2}}, k=1,3,5, \ldots$ to be antisymmetric in the sense

$$
\int d^{d} x A \square^{\frac{k}{2}} B=-\int d^{d} x B \square^{\frac{k}{2}} A+\text { boundary terms, }
$$


then the formula (44) provides a boundary term.

The $h, h^{m}, h^{m n}$-terms are what we call conformal spin-0, 1, 2 theories in d dimensions, respectively. In $d=4$, spin-1 and spin-2 theories are the linearized Maxwell and Weyl gravity actions. Note also that, in $d=4$, the scalar $h$ is auxiliary. At the quadratic level $h=0$ on-shell, while at nonlinear level $h$ is expressed via the higher rank fields and their derivatives.

Let us make a remark. The general action (30) is constructed in such a way that the Weyl weight of the monomials in curvatures $F_{m n}, C_{m n k l}$ is compensated either by the Weyl weight of the powers of the dilaton $D$ or by the Weyl weight of the operators which contain covariant derivatives, like $\square^{k}+o_{k}(R)$. These are the latter "derivative" terms that survive in the limit $D \rightarrow 0$ and which expansion gives rise to the quadratic conformal theories (44). In particular, the $s=0$ quadratic action comes not from the "conventional dilaton's kinetic term" $k=1$ in the first row of (30) but rather from $k=\frac{d}{2}-2$ one (the only case when these terms coincide is $d=6$ ). On the other hand, let us redefine the dilaton as

$$
D(x)=\phi^{l}(x)
$$

This change of dynamical variables is degenerate at the vacuum point $D=0$, therefore, the quadratic theories of the fluctuations of $D$ and $\phi$ are in general non-equivalent. The mechanism that ensures the lack of equivalence at the quadratic level is that, for different $l$, the quadratic actions for the fluctuations of $\phi$ originate from different terms in (30). In fact, the terms which give the non-vanishing contribution to the quadratic action of the fluctuation of $\phi$ are those with $k=\frac{d}{2}-\frac{2}{l}$, then the quadratic action is proportional to $\int d^{d} x \phi \square^{k} \phi$. In particular, for the redefinition (46) with $l=\frac{4}{d-2}$ one has $k=1$ and the "conventional" action of the massless scalar.

The last issue we would like to discuss in this section is the global invariance. Usually the global symmetries arise as a subgroup of gauge transformations preserving the vacuum. In the case of gauge transformations (29) and the vacuum (39), the global transformations are conformal transformations, The third row is the definition of the conformal Killing vector $\xi^{m}$, with general solution of the form

$$
\xi^{a}(x)=b^{a}+2 b^{a b} x_{b}+c x^{a}+x^{2} c^{a}-2 x^{a} x^{b} c_{b}, \alpha=-c+2 c^{a} x_{a},
$$

where $b^{a}, b^{a b}=-b^{b a}, c, c^{a}$ parameterize Poincaré translations, Lorentz rotations, dilations and special conformal transformations, which altogether generate the Lie algebra so $(d-$ $q+1, q+1)$. These transformations do not change the vacuum (39) because dilation of the flat metric due to diffeomorphisms is compensated by a Weyl dilation. 


\subsection{Linearized data.}

\subsubsection{Free conformal higher spin theories}

After the perturbative expansion over a conformally flat vacuum, the quadratic part of the action of our model breaks down as theory of free scalar plus a sum of conformal higher spin actions for conformal spin $s=1,2,3, \ldots$

Let us describe these actions in more detail.

Given $s=1,2,3, \ldots$ consider symmetric traceless tensor field

$$
\varphi^{a(s)}(x), \varphi_{b}^{a(s-2) b}=0
$$

on a $d$-dimensional flat space $\mathbf{R}^{d-q, q}$, equipped with a flat metric $\eta_{a b}$. Consider gauge transformations

$$
\delta \varphi^{a(s)}=\text { Traceless part of } \partial^{a} \varepsilon^{a(s-1)}(x),
$$

where $\varepsilon^{a(s-1)}(x)$ is an arbitrary smooth traceless tensor field with a compact support, and wonder if there exists a gauge-invariant and Poincaré-invariant quadratic action $\mathcal{A}_{s}\left[\varphi_{s}\right]$. The answer is yes, and the action has the form

$$
\mathcal{A}_{s}\left[\varphi_{s}\right]=\int d^{d} x \varphi^{a(s)} P_{a(s) b(s)}(\partial) \varphi^{b(s)}
$$

where $P_{a(s) b(s)}(\partial)$ is a Poincaré-invariant differential operator, satisfying set of identities

$$
P_{a(s) b(s)}=P_{b(s) a(s)}, P_{c a(s-2) b(s)}^{c}=0 \quad, \quad P_{a(s-1) c b(s)} \partial^{c}=0 .
$$

The Poincaré-invariant solution to the equations (51) appears to have the form

$$
P_{a(s) b(s)}(\partial)=\mathcal{P}_{a(s) b(s)}(\partial) f(\square)
$$

where operator $\mathcal{P}_{a(s) b(s)}(\partial)$ is of $2 s$-order in $x$-derivatives and $f$ is an arbitrary function of $\square=\partial_{a} \partial^{a}$. The generating formula (83) for all $s$ is exhibited in Sec.(3.1).

As far as one deals with boson actions the choice of the metric signature is inessential, and the actions look identical for any signature, being hidden in the definition of the metric.

Needless to say, the gauge invariance of the actions (50,51) holds in arbitrary dimension, as a consequence of identities (51). In this paper we demonstrate that, in even dimensions $d>2$, the actions $(50,51,52$ ) are invariant w.r.t. conformal algebra in so $(d-q+1, q+1)$, under the choice

$$
f(\square)=\square^{\frac{d}{2}-2}
$$


Therefore, for any even $d$, it is reasonable to name the theories $(50,51,52,53)$ spin-s conformal higher spin theories. If the factor $f(\square)$ is arbitrary we refer corresponding model as traceless higher spin theory. Note that in $d=4$ the conformal spin-0 field is an auxiliary field, the fact that will play an important role below. For odd $d$, it is worth defining operators $\square \frac{d}{2}$ to be antisymmetric in the sense of Eq. (45) then the quadratic action (50) is a boundary term.

In $d=4$, these models were introduced by Fradkin and Tseytlin [1]. Their supersymmetric extensions and cubic order interactions were studied by Fradkin and Linetsky [14].

\subsubsection{Free higher spin theories and the higher spin problem}

The conformal higher spin theories may be considered as a truncation of higher spin theories. The latter are formulated, at the linearized level, in terms of two symmetric traceless tensor fields $\varphi^{a(s)}(x), \chi^{a(s-2)}$ subject to the following gauge transformations

$$
\begin{gathered}
\delta \varphi^{a(s)}=\text { Traceless part of } \partial^{a} \varepsilon^{a(s-1)}(x) \\
\delta \chi^{a(s-2)}=\frac{s-1}{2 s-4+d} \partial_{b} \varepsilon^{b a(s-2)}(x),
\end{gathered}
$$

where the gauge parameter $\varepsilon^{a(s-1)}(x)$ is an arbitrary traceless tensor field, just like in the conformal higher spin theory case. The Poincaré-invariant and gauge-invariant action $\mathcal{A}_{s}\left[\varphi_{s}, \chi_{s-2}\right]$ is fixed unambiguously by the requirement of absence of higher (more than 2) derivatives in the Lagrangian.

$$
\begin{gathered}
\mathcal{A}_{s}\left[\varphi_{s}, \chi_{s-2}\right]=\frac{(-)^{s}}{2} \int d^{d} x\left\{\partial_{n} \phi_{m_{1} \ldots m_{s}} \partial^{n} \phi^{m_{1} \ldots m_{s}}\right. \\
-\frac{1}{2} s(s-1) \partial_{n} \phi_{k m_{1} \ldots m_{s-2}}^{k} \partial^{n} \phi_{k}^{k}{ }_{k}^{m_{1} \ldots m_{s-2}} \\
+s(s-1) \partial_{n} \phi_{k m_{1} \ldots m_{s-2}}^{k} \partial_{l} \phi^{n l m_{1} \ldots m_{s-2}}-s \partial_{n} \phi_{m_{1} \ldots m_{s-1}}^{n} \partial_{k} \phi^{k m_{1} \ldots m_{s-1}} \\
\left.-\frac{1}{4} s(s-1)(s-2) \partial_{n} \phi^{k}{ }_{k n m_{1} \ldots m_{s-3}} \partial_{r} \phi_{l}^{l}{ }_{l}^{r m_{1} \ldots m_{s-3}}\right\}
\end{gathered}
$$

where double-traceless field $\phi^{m(s)}$ is

$$
\phi^{m(s)}=\varphi^{m(s)}+\eta^{m(2)} \chi^{m(s-2)}
$$

These models were introduced by Fronsdal in 4d [2], on the other hand, the form of the actions appears to be independent of the dimension [15]. They admit generalization to $d$-dimensional anti de Sitter space [16], [17], [18], [19], [57]. We will refer these models as 
Fronsdal theories of spin s. For Minkowski signature, for any $s$ Fronsdal theories describe on-shell the unitary irreducible representation of the Poincaré (anti- de Sitter) group and therefore some free particle, and for $s=0,1,2$, they are just free scalar, photon and graviton, respectively. A natural question arises is there a consistent nonlinear theory, describing interaction of higher spin particles $s \geq 2$ to low spin particles $(s \leq 2$ : scalars, photons, gravitons) and among themselves. This task is highly nontrivial, as almost any naive attempt of introducing interaction in Fronsdal theories breaks down their extended gauge invariance (54),(55) thus making the nonlinear models inconsistent. Despite the progress in the last two decades [20]-23], [24], 25] this problem has not got the convincing solution.

We see that, at the linearized level, gauge parameters in the spin- $s$ conformal higher spin theories and spin-s Fronsdal theories are the same, moreover, the gauge transformations are the same for $\varphi_{s}$ field. The difference is that Fronsdal models possess an additional symmetric traceless tensor field $\chi^{a(s-2)}$ which we call compensator. In this sense, the conformal higher spin theory arises as a limit of higher spin theory when the compensator decouples.

More precisely, it is natural to anticipate existence of a united nonlinearized model which, being expanded around certain vacuum, reproduces in quadratic approximation the sum of actions like

$$
\mathcal{A}=\sum_{s=0}^{\infty}\left(l^{2} \mathcal{A}_{s}\left[\varphi_{s}, \chi_{s-2}\right]+\{\ldots\}+l^{d-4+2 s} \mathcal{A}_{s}\left[\varphi_{s}\right]\right)
$$

where $l$ is a parameter counting number of derivatives and $\{\ldots\}$ stands for another higher derivative gauge-invariant terms depending on $\varphi_{s}, \chi_{s-2}$, with number of derivatives being less than $d-4+2 s$. Provided $s$ is fixed, in the limit $l \rightarrow \infty$ the $l^{4-d+2 s}$ terms which depend solely on $\varphi_{s}$ dominate, and the theory describes conformal higher spin fields. What we have constructed in this paper is the nonlinear generalization of the sum of these terms. We suppose analogous decoupling phenomena arise at the nonlinear level. In this sense, the construction of the full conformal higher spin theory, achieved in this paper, is a step towards the solution of the higher spin problem.

Note that higher spin theory is believed not to admit flat vacua, and higher spin interactions are being formulated in anti-de Sitter space [3]. As we will show in the paper the conformal higher spin theory possesses conformally flat vacua with an arbitrary value of the conformal factor of the metric. As anti-de Sitter space is conformally flat, our results do not come to contradiction with existing results on higher spin interactions. 


\subsubsection{On AdS/CFT "higher spin massless fields in $A d S_{d+1} /$ conformal higher spin fields in $d$-dimensional Minkowski space"}

Conformal higher spin fields and Fronsdal fields have one more important interrelation. Namely, conformal higher spin fields in $d$ dimensions arise as boundary values of $A d S_{d+1}$ Fronsdal fields. Let us comment on this point, following Refs [10], [11]. Let $d$ be even. Consider free massless spin- $s$ theory in $A d S_{d+1}$ and evaluate the action of this model in terms of solutions of Dirichlet like problem in terms of the "boundary values" of $\Phi_{s}$. According to general $A d S / C F T$ principles [6], one arrives at a quadratic nonlocal functional of "boundary values" $\varphi_{s}(x)$ of $\Phi_{s}(x, z)$, which is a generating functional of the two-point function of primary operators $\mathcal{O}_{s}$ in a $d$-dimensional conformal field theory, with mass dimension $\Delta=E$, where $E$ is a lowest eigenvalue of the $A d S$ energy operator in the bulk theory. For the bulk theory in question one has [17]

$$
E=s+d-2
$$

and therefore the coupling

$$
S=\int d^{d} x \varphi_{s}(x) \mathcal{O}_{s}(x)
$$

is conformally invariant provided conformal dimension of $\varphi_{s}$ is, in mass units,

$$
\left[\varphi_{s}\right]=2-s
$$

wherefrom one expects the result of calculation of $A d S$ action to have a form

$$
\tilde{\mathcal{A}}_{s}\left[\varphi_{s}\right]=\int d^{d} x d^{d} x^{\prime} \frac{\varphi_{s}(x) P\left(x-x^{\prime}\right) \varphi_{s}\left(x^{\prime}\right)}{\left(\left(x-x^{\prime}\right)^{2}+\epsilon^{2}\right)^{s+d-2}}
$$

where $\epsilon$ is a regulator which restricts the limits of integration in $A d S_{d+1}$ and $P\left(x-x^{\prime}\right)$ is a kernel of an operator of zero mass dimension.

In the limit $\epsilon \rightarrow 0$ one extracts the logarithmically divergent part of this expression to be, schematically,

$$
\sim \ln \epsilon^{2} \int d^{d} x \varphi_{s}(x) \square^{\frac{d}{2}-2+s} P(\partial) \varphi_{s}(x),
$$

that is in accord with the structure of conformal higher spin theory action (50 52), (53). Actually, the last expression is gauge invariant w.r.t. transformations (49) (the latter arise as a consequence of gauge transformations for $A d S_{d+1}$ massless fields) and coincides with the action of free spin- $s$ conformal higher spin theory. Thus, in even dimensions $d$, free conformal higher spin theories action present the logarithmically divergent terms in the free actions of $A d S_{d+1}$ massless fields expressed in terms of the solutions of Dirichlet like problem. 


\section{Technical digression. Deformed traceless higher spin theories}

As we stated above, our theory reproduces the sum of conformal higher spin models in the framework of a perturbative expansion around a conformally flat vacuum. In that framework, conformal higher spin models reveal themselves not in their original form described in the previous subsection but in the form of deformed traceless higher spin theories, the latter being equivalent to the former.

To show this equivalence and to exhibit the map "deformed traceless higher spin theories $\leftrightarrow$ conformal higher spin theories" we have to provide a rather extensive technical digression we devote this section to. We describe deformed traceless higher spin theories and show that, in an appropriate basis of fields, the deformed traceless higher spin theories are just the conformal higher spin theories of the previous subsection.

The results of this section will be very useful in analyzing perturbative expansion of our theory around a conformally flat background.

\subsection{Deformed traceless higher spin theories.}

In fact, here we recall and update the results of our paper [56]. By definition, a deformed traceless higher spin theory is characterized by a Poincaré-invariant quadratic action built from the infinite number of symmetric tensor fields

$$
h^{m_{1} \ldots m_{k}}(x), k=0,1,2,3, \ldots
$$

subject to gauge transformations of the form $\left(\eta^{m n}\right.$ is Minkowski metric)

$$
\delta h^{m(k)}(x)=-2 \mu^{2} a^{m(k)}(x)-2 \eta^{m(2)} a^{m(k-2)}(x)-2 \partial^{m} \epsilon^{m(k-1)}(x),
$$

with the infinite set of gauge parameters, symmetric tensors

$$
\epsilon^{m_{1} \ldots m_{k}}, a^{m_{1} \ldots m_{k}}, k=0,1,2,3, \ldots
$$

which are required to have a compact support in $x$-space. $\mu^{2}$ is a general Poincaré-invariant scalar operator, being a general function of $\square$ :

$$
\mu^{2}=\mu^{2}(\square)
$$

Deformed higher spin theories are parameterized by integer $s=0,1,2, \ldots$ up to the arbitrariness in multiplying the wave operator by an arbitrary function of $\square$. 
The gauge transformations may be written in generating form if one introduces power series, depending on a covector variable $p_{m}$,

$$
\begin{aligned}
& h(x, p)=\sum_{k=0}^{\infty} h^{m_{1} \ldots m_{k}}(x) p_{m_{1}} \ldots p_{m_{k}} \\
& \epsilon(x, p)=\sum_{k=0}^{\infty} \epsilon^{m_{1} \ldots m_{k}}(x) p_{m_{1}} \ldots p_{m_{k}} \\
& a(x, p)=\sum_{k=0}^{\infty} a^{m_{1} \ldots m_{k}}(x) p_{m_{1}} \ldots p_{m_{k}} .
\end{aligned}
$$

The gauge transformations (65) read

$$
\delta h(x, p)=-2\left(p^{2}+\mu^{2}\right) a(x, p)-2 p \partial_{x} \epsilon(x, p)
$$

Note that provided $\mu^{2}=0$ the $a$-gauge transformations in (65) allow one to gauge away all the traces of tensors $h^{m_{1} \ldots m_{k}}$ and to set $h^{m_{1} \ldots m_{k}}$ traceless,

$$
h^{m_{1} \ldots m_{k}}=\varphi^{m_{1} \ldots m_{k}}, \varphi_{n}^{m_{1} \ldots m_{k-2} n}=0
$$

Then the invariant action should be expressed in terms of $\varphi$ 's. In terms of $\varphi$ 's, the gauge transformations (65) read

$$
\begin{gathered}
\delta \varphi^{m(k)}(x)=\text { Traceless part of } \partial^{m} \varepsilon^{m(k-1)}(x), \\
\varepsilon^{m(k-1)}(x)=-\frac{1}{2} \text { Traceless part of } \epsilon^{m(k-1)}(x),
\end{gathered}
$$

that coincides with gauge transformations for free conformal higher spin theories of the Sec. (2.2.1). Therefore, the quadratic gauge-invariant Poincaré-invariant action which depends on fields (64) subject to the gauge transformations (65, 66) is, in the case $\mu^{2}=0$, the sum of free conformal higher spin theories (50).

Let us see what happens when $\mu^{2} \neq 0$. Consider a general Poincaré-invariant quadratic action for the fields (64). The general form of the action is

$$
A_{P}[h]=\sum_{k=0, k^{\prime}=0}^{\infty} \int d^{d} x h^{m_{1} \ldots m_{k}}(x) P_{\left\{m_{1} \ldots m_{k} \mid n_{1} \ldots n_{k^{\prime}}\right\}}\left(\partial_{l}\right) h^{n_{1} \ldots n_{k^{\prime}}}(x)
$$

where $P_{\left\{m_{1} \ldots m_{k} \mid n_{1} \ldots n_{k}\right\}}\left(\partial_{l}\right)$ are some (pseudo)differential operators constructed from the partial derivative $\partial_{m}$ and the Minkowski metric, they are also allowed to contain any function of $\square$. The operator $P_{\{m(k) \mid n(k)\}}\left(\partial_{l}\right)$ is to be symmetric, therefore

$$
P_{\left\{m(k) \mid n\left(k^{\prime}\right)\right\}}\left(-\partial_{l}\right)=P_{\left\{n\left(k^{\prime}\right) \mid m(k)\right\}}\left(\partial_{l}\right)
$$


Let us require the action (72) to be invariant w.r.t. gauge transformations (65),69). The gauge invariance of the action (72) is equivalent to the following set of identities on the components of the wave operator:

$$
\begin{gathered}
P_{\left\{l_{1}, l_{2} \ldots l_{k} \mid n_{1} \ldots n_{k^{\prime}-1} r\right\}} \partial^{r}=0 ; \\
\eta^{a b} P_{\left\{l_{1} \ldots l_{k} \mid n_{1} \ldots n_{k^{\prime}-2} a b\right\}}+\mu^{2} P_{\left\{l_{1} \ldots l_{k} \mid n_{1} \ldots n_{k^{\prime}-2}\right\}}=0 ; \forall k, k^{\prime},
\end{gathered}
$$

and analogously for $n \leftrightarrow l$.

This infinite system of identities may be easily solved in a generating framework. Introduce the power series of two variables $q^{m}, q^{\prime m}$

$$
P\left(q, q^{\prime}, \partial\right)=\sum_{k=0, k^{\prime}=0}^{\infty} \frac{1}{k ! k^{\prime} !} q^{n_{1}} \ldots q^{n_{k^{\prime}}} q^{\prime m_{1}} \ldots q^{\prime m_{k}} P_{\left\{m_{1} \ldots m_{k} \mid n_{1} \ldots n_{k^{\prime}}\right\}}\left(\partial_{l}\right) \text {. }
$$

This function of three vector-like variables encodes the full information about the quadratic action (72) and moreover, given $P\left(q, q^{\prime}, \partial\right)$ the action is easily recovered by means of the formula

$$
A_{P}[h]=\int d^{d} x\left\{h(x, p) P\left(\frac{\overleftarrow{\partial}}{\partial p}, \frac{\vec{\partial}}{\partial p^{\prime}}, \partial\right) h\left(x, p^{\prime}\right)\right\}_{p=p^{\prime}=0}
$$

The infinite system of identities (74) is equivalent to two equations on $P\left(q, q^{\prime}\right)$ :

$$
\eta^{m n} \frac{\partial}{\partial q^{m}} \frac{\partial}{\partial x^{n}} P\left(q, q^{\prime}\right)=0,\left(\eta^{m n} \frac{\partial}{\partial q^{m}} \frac{\partial}{\partial q^{n}}+\mu^{2}\right) P\left(q, q^{\prime}\right)=0,
$$

The equation (73) turns into

$$
P\left(q, q^{\prime}, \partial\right)=P\left(q^{\prime}, q,-\partial\right)
$$

and therefore one gets also

$$
\eta^{m n} \frac{\partial}{\partial q^{\prime m}} \frac{\partial}{\partial x^{n}} P\left(q, q^{\prime}\right)=0,\left(\eta^{m n} \frac{\partial}{\partial q^{\prime m}} \frac{\partial}{\partial q^{\prime n}}+\mu^{2}\right) P\left(q, q^{\prime}\right)=0 .
$$

The equations (77)-(79) constitute the full set of conditions for gauge invariance of the action (72).

The general solution of the gauge invariance constraints (77-79) has been obtained in [56]. It has the form

$$
\begin{gathered}
P\left(q, q^{\prime}, \partial\right)=\sum_{s=0}^{\infty} \tilde{\alpha}_{s}(\square) P_{\mu}^{(s)}\left(q, q^{\prime}, \partial\right) \\
P_{\mu}^{(s)}\left(q, q^{\prime}, \partial\right)=\left(\rho \rho^{\prime}\right)^{-\frac{d-3}{2}} J_{s+\frac{d-3}{2}}\left(\frac{\mu}{\sqrt{\square}} \rho\right) J_{s+\frac{d-3}{2}}\left(\frac{\mu}{\sqrt{\square}} \rho^{\prime}\right) \sum_{k=0}^{\left[\frac{s}{2}\right]}\left(\frac{\tau}{\rho \rho^{\prime}}\right)^{2 k+\varsigma} r_{2 k+\varsigma, s+\frac{d-3}{2}} \\
\rho^{2}=\square q^{2}-(q \partial)^{2} ; \rho^{\prime 2}=\square q^{\prime 2}-\left(q^{\prime} \partial\right)^{2} ; \tau=\square\left(q q^{\prime}\right)-(q \partial)\left(q^{\prime} \partial\right),
\end{gathered}
$$


where $\tilde{\alpha}_{s}$ are arbitrary functions of $\square$ and $J_{\nu}(z)$ are Bessel's functions of the first kind (see App. (因)). Here $\varsigma=0,1$ if $s$ is even or odd, correspondingly, while

$$
\begin{aligned}
r_{2 k+\varsigma, s+\frac{d-3}{2}}= & \frac{(-1)^{k}}{(2 k+\varsigma) !} \frac{(s-\varsigma) ! !(s+\varsigma+d+2 k-5) ! !}{(s-\varsigma-2 k) ! !(s+\varsigma+d-5) ! !} r_{\varsigma, s+\frac{d-3}{2}} \equiv \\
& \equiv c_{2 k+\varsigma, s+\frac{d-3}{2}} r_{\varsigma, s+\frac{d-3}{2}},
\end{aligned}
$$

$r_{\varsigma, s+\frac{d-3}{2}}$ is an arbitrary "constant", i.e. arbitrary function of $\square$, which is to be absorbed by redefinition of $\tilde{\alpha}_{s}$, so without loss of generality we set it equal to 1 .

Note that the solution contains only even powers of $\rho, \rho^{\prime}$ as it should. It appears the sums by $k$ in (80) are expressed in terms of Gegenbauer polynomials [52] as follows

$$
\sum_{k=0}^{\left[\frac{s}{2}\right]}\left(\frac{\tau}{\rho \rho^{\prime}}\right)^{2 k+\varsigma} r_{2 k+\varsigma, s+\frac{d-3}{2}}=\frac{(-)^{\left[\frac{s}{2}\right]} 2^{-s}(s-\varsigma) !(2 s+d-5) ! ! \Gamma\left(\frac{d-3}{2}\right)}{(s+\varsigma+d-5) ! ! \Gamma\left(s+\frac{d-3}{2}\right)} \mathcal{C}_{s}^{\frac{d-3}{2}}\left(\frac{\tau}{\rho \rho^{\prime}}\right)
$$

where Gegenbauer polynomials $\mathcal{C}_{s}^{\frac{d-3}{2}}$ are described in Appendix $(\mathbb{E})$. The overall factor is to be absorbed by $\tilde{\alpha}_{s}$ so the general solution reads

$$
\begin{gathered}
P\left(q, q^{\prime}, \partial\right)=\sum_{s=0}^{\infty} \alpha_{s}(\square) P_{\mu}^{(s)}\left(q, q^{\prime}, \partial\right) \\
P_{\mu}^{(s)}\left(q, q^{\prime}, \partial\right)=\frac{\Gamma\left(\frac{d-3}{2}\right)}{\Gamma\left(s+\frac{d-3}{2}\right)}\left(\rho \rho^{\prime}\right)^{-\frac{d-3}{2}} J_{s+\frac{d-3}{2}}\left(\frac{\mu}{\sqrt{\square}} \rho\right) J_{s+\frac{d-3}{2}}\left(\frac{\mu}{\sqrt{\square}} \rho^{\prime}\right) \mathcal{C}_{s}^{\frac{d-3}{2}}\left(\frac{\tau}{\rho \rho^{\prime}}\right),
\end{gathered}
$$

that presents a refinement of the results of our paper [56].

To clarify the meaning of the parameter $s$ it is worth taking the limit $\mu^{2} \rightarrow 0$. As it is argued above, in this limit the action should describe conformal higher spin theory in the original form (50 52). This is really the case. Indeed, as $\mu^{2}=0$, only first term of the Bessel's series in (83) survives, so one gets,

$$
P_{0}^{(s)}\left(q, q, \partial^{\prime}\right) \sim \sum_{k=0}^{\left[\frac{s}{2}\right]} \tau^{2 k+\varsigma}\left(\rho \rho^{\prime}\right)^{s-\varsigma-2 k} c_{2 k+\varsigma, s+\frac{d-3}{2}} .
$$

As it is clear from the very definition $(75)$, a term $I_{a, b, c} \sim \tau^{a} \rho^{2 b} \rho^{2 c}$ in the solution leads to the corresponding operator in the action (72), which contains $2 a+2 b+2 c x$-derivatives, and involve tensor fields of ranks $a+2 b$ and $a+2 c$. Applying this account to the last equation we see that the corresponding theory contains terms $I_{2 k+\varsigma,\left[\frac{s}{2}\right]-k,\left[\frac{s}{2}\right]-k}$ for $k=0, \ldots,\left[\frac{s}{2}\right]$ and thereby describes theory of symmetric tensor fields of rank-s only, with operators of only $2 s$-th order in $x$-derivatives (for $n=\varsigma=0$, the solution is just a constant).

Let us make an important remark. Due to invariance w.r.t. $a$-transformations, the actions (72), (329) depend only upon the special traceless combinations of $h^{m(k)}$, described in the Appendix $(\overline{\mathrm{G}})$ (one traceless tensor for each rank $s$ ). Specifically, $h(x, p)$ may be represented as

$$
h(x, p)=\varphi(x, p)+\left(p^{2}+\mu^{2}\right) \chi(x, p)
$$


where $\chi(x, p)$ is arbitrary power series in $p_{m}$ while $\varphi$ is a traceless power series:

$$
\varphi(x, p)=\sum_{s=0}^{\infty} \varphi^{m(s)}(x) p_{m_{1}} \ldots p_{m_{s}} ; \varphi_{n}{ }^{n m(s-2)}=0 .
$$

Then it is clear the action may be written in terms of $\varphi$ by making substitution $h(x, p) \mapsto$ $\varphi(x, p)$ in the action (72), after the substitution, the terms $q^{2}, q^{\prime 2}$ in the generating function $P\left(q, q^{\prime}, \partial\right)$ (329) may be dropped. This simplify the form of $\rho \mapsto \pm i(q \partial), \rho^{\prime} \mapsto \pm i\left(q^{\prime} \partial\right)$. In this basis, gauge transformations of $\varphi^{m(s)}(x)$ appear to depend only upon the special traceless parts of $\epsilon$ (544,546).

\subsection{Dressing and Undressing maps}

The general solution of gauge invariance constraints (83) exhibits what we call dressing. Given a function of two vector-like variables $a^{m}, b_{m} U(a, b)$, if one "dresses" $P\left(q, q^{\prime}, \partial\right)$ like

$$
P_{U}\left(q, q^{\prime}, \partial\right)=U(q, \partial) U\left(q^{\prime}, \partial\right) P\left(q, q^{\prime}, \partial\right) \Leftrightarrow
$$

then new operator $P_{U}\left(q, q^{\prime}, \partial\right)$ already does not satisfy the same equations as for $P\left(q, q^{\prime}, \partial\right)$. However, if this change is accompanied by the following "undressing" of gauge fields

$$
h(x, p)=U\left(\frac{\partial}{\partial p}, \partial\right) h_{U}(x, p)
$$

then it results in the same theory, i.e.

$$
A_{P_{U}}\left[h_{U}\right]=A_{P}[h]
$$

This property of "dressing" plays an important role in our considerations.

Consider spin-s deformed model with generating function $P_{\mu}^{(s)}(83)$. It is seen that

$$
\begin{gathered}
P^{(s)}\left(q, q^{\prime}, \partial\right)=U_{s+\frac{d-3}{2}}\left(\frac{\mu}{\sqrt{\square}} \rho\right) U_{s+\frac{d-3}{2}}\left(\frac{\mu}{\sqrt{\square}} \rho^{\prime}\right) \mathcal{P}^{(s)} \\
\mathcal{P}^{(s)}=\frac{\Gamma\left(\frac{d-3}{2}\right)}{\Gamma\left(s+\frac{d-3}{2}\right)} \square^{\frac{d}{2}-2}\left(\rho \rho^{\prime}\right)^{s} \mathcal{C}_{s}^{\frac{d-3}{2}}\left(\frac{\tau}{\rho \rho^{\prime}}\right)
\end{gathered}
$$

where

$$
U_{\nu}(z) \equiv z^{-\nu} J_{\nu}(z), \quad U_{\nu}(0)=\left(\frac{1}{2}\right)^{\nu} \frac{1}{\Gamma(\nu+1)},
$$

and therefore this model is related to ordinary conformal higher spin model by the dressing map with the dressing operator

$$
U=U_{s+\frac{d-3}{2}}\left(\frac{\mu}{\sqrt{\square}} \rho\right)
$$

Note that this operator contains only even powers of $\rho$ so the corresponding dressing map is well-defined in the space of power series in momenta. 
It is seen that up to a constant multiplier $\mathcal{P}^{(s)}$ is the $\mu \rightarrow 0$ limit of $P^{(s)}$, therefore, it satisfies the set of constraints obtained as $\mu \rightarrow 0$ limit of the constraints (77-79):

$$
\begin{gathered}
\partial_{q}^{2} \mathcal{P}^{(s)}\left(q, q^{\prime}, \partial\right)=0 ; \partial_{q^{\prime}}^{2} \mathcal{P}^{(s)}\left(q, q^{\prime}, \partial\right)=0 \\
\partial_{q} \partial \mathcal{P}^{(s)}\left(q, q^{\prime}, \partial\right)=0 ; \partial_{q^{\prime}} \partial \mathcal{P}^{(s)}\left(q, q^{\prime}, \partial\right)=0
\end{gathered}
$$

Besides this, $\mathcal{P}^{(s)}$ is a homogeneous polynomial of order $s$ in $q$ and, separately, in $q^{\prime}$,

$$
q \partial_{q} \mathcal{P}^{(s)}\left(q, q^{\prime}, \partial\right)=s \mathcal{P}^{(s)}\left(q, q^{\prime}, \partial\right) ; q^{\prime} \partial_{q^{\prime}} \mathcal{P}^{(s)}\left(q, q^{\prime}, \partial\right)=s \mathcal{P}^{(s)}\left(q, q^{\prime}, \partial\right)
$$

Summing up, the sum of deformed spin-s theories (83) may be rewritten as follows

$$
\begin{gathered}
A_{P}[h]=\sum_{s=0}^{\infty} \int d^{d} x\left\{\chi^{(s)}(x, p) \alpha_{s}(\square) \mathcal{P}^{(s)}\left(\frac{\overleftarrow{\partial}}{\partial p}, \frac{\vec{\partial}}{\partial p^{\prime}}, \partial\right) \chi^{(s)}\left(x, p^{\prime}\right)\right\}_{p=p^{\prime}=0} \\
\chi^{(s)}=U_{s+\frac{d-3}{2}}\left(\frac{\mu}{\sqrt{\square}} \vec{\rho}\right) h(x, p),
\end{gathered}
$$

where

$$
\vec{\rho}=\rho\left(q \rightarrow \frac{\vec{\partial}}{\partial p}\right)
$$

Furthermore one notes that each spin- $s$ contribution $\int \ldots \chi^{(s)} \ldots \chi^{(s)}$ in $(\overline{96})$ depends on only rank-s component of $\chi^{(s)}$, because $\mathcal{P}^{(s)}$ is a homogeneous polynomial of order $s$ in $q$ and, separately, in $q^{\prime}$. Therefore, the sum of spin-s actions is rewritten in the manner

$$
\begin{gathered}
A_{P}[h]=\sum_{s=0}^{\infty} \int d^{d} x\left\{\phi_{s}(x, p) \alpha_{s}(\square) \mathcal{P}^{(s)}\left(\frac{\overleftarrow{\partial}}{\partial p}, \frac{\vec{\partial}}{\partial p^{\prime}}, \partial\right) \phi_{s}\left(x, p^{\prime}\right)\right\}_{p=p^{\prime}=0}=\sum_{s=0}^{\infty} \mathcal{A}_{s}\left[\phi_{s}\right] \\
\phi_{s} \equiv \chi_{s}^{(s)}=\text { projection to the rank- } s \text { subspace of the power series } \chi^{(s)}(x, p) \\
p \frac{\partial}{\partial p} \phi_{s}=s \phi_{s} .
\end{gathered}
$$

One observes that the sum of the deformed conformal higher spin models is always expressed via the set of symmetric tensor fields $\phi^{m_{1} \ldots m_{k}}(x)$ which enter the action via

$$
\phi_{s}(x, p)=\phi^{m_{1} \ldots m_{k}}(x) p_{m_{1}} \ldots p_{m_{s}}
$$

This set of fields is the substitute of the initial one $h^{m_{1} \ldots m_{k}}(x)$ which makes up the power series $h(x, p)$, in the sense that the quadratic action is expressed entirely in terms of the "undressed" variables $\phi^{m_{1} \ldots m_{k}}(x)$ expressed via original fields $h^{m_{1} \ldots m_{k}}(x)$ and their $x$-derivatives up to an infinite order by means of the formulae (96, 98). In terms of the undressed fields the actions (98) have the simple form of conformal higher spin theories. Indeed, consider the action $\mathcal{A}_{s}\left[\phi_{s}\right]$ separately. The constraints (93-94) ensure gauge invariance of the action w.r.t. gauge transformations

$$
\delta \phi_{s}(x, p)=-2 p^{2} a_{s-2}(x, p)-2 p \partial_{x} \epsilon_{s-1}(x, p),
$$


where $a_{s-2}, \epsilon_{s-1}$ are homogeneous polynomials in momenta of degrees $s-2, s-1$, respectively, or, in terms of components,

$$
\delta \phi^{m(s)}(x)=-2 \eta^{m(2)} a^{m(s-2)}(x)-2 \partial^{m} \epsilon^{m(s-1)}(x) .
$$

This is nothing but the gauge laws of $\mu=0$ theories. As we noted above, the algebraic $a_{s-2}$-invariance manifests the decoupling of the trace part of $\phi^{m(s)}$, in other words, the action $\mathcal{A}_{s}\left[\phi_{s}\right]$ depends on the traceless part of $\phi^{m(s)}$ only,

$$
\mathcal{A}_{s}\left[\phi_{s}\right]=\mathcal{A}_{s}\left[\varphi_{s}\right], \quad \varphi^{m(s)}=\text { Traceless part of } \phi^{m(s)}(x)
$$

In terms of $\varphi_{s}$, the remaining $\epsilon_{s-1}$-gauge invariance reads

$$
\begin{gathered}
\delta \varphi^{m(s)}=\text { Traceless part of } \partial^{m} \varepsilon^{m(s-1)}(x) \\
\varepsilon^{m(s-1)}=-\frac{1}{2} \text { Traceless part of } \epsilon^{m(s-1)} .
\end{gathered}
$$

These gauge transformations coincide with (49), which is the starting point of the undeformed conformal higher spin theories we have started with. Needless to say, the action $\mathcal{A}_{s}\left[\varphi_{s}\right]$ is nothing but the action of $\mu^{2}=0$ conformal higher spin theories (50-52). Therefore, the deformed traceless higher spin theory, expressed in terms of the "undressed" variables $\varphi_{s}$ is just free conformal higher spin theory.

\subsection{Reconstruction map}

The quadratic approximation of the conformal higher spin theories looks most simply in terms of the undressed variables $\phi_{s}$. On the other hand, for our purposes it is vital to find the inverse map from the data provided by the set of undressed "pure spin" variables $\phi_{s}$ to the original ones contained in the fluctuation $h(x, p)$. The matter is that the full action as well as closed nonlinearized gauge transformations will be formulated below in terms of the dressed fields $h$ 's, on the other hand, the original "conformal higher spin fields" are the undressed ones $\phi$ 's. In order to get interaction vertices in terms of the undressed fields one has to find the vertices in terms of $h$ and then to represent $h$ via $\phi$.

Proposition 1 Given the "undressing" map

$$
\phi_{s} \equiv \chi_{s}^{(s)}=\text { projection to the subspace of } s \text {-th degree in } p_{m} \text { of } U_{s+\frac{d-3}{2}}\left(\frac{\mu}{\sqrt{\square}} \vec{\rho}\right) h(x, p) \text {, }
$$

the equation holds

$$
\sum_{s=0}^{\infty} \frac{i^{s}}{s !} h_{s}=2^{\frac{d-3}{2}} \Gamma\left(\frac{d-3}{2}\right) \sum_{s=0}^{\infty} i^{s}\left(s+\frac{d-3}{2}\right)\left(\frac{\mu}{\sqrt{\square}} \vec{\rho}\right)^{s} \mathcal{C}_{s}^{\frac{d-3}{2}}\left(\frac{\sqrt{\square}}{\mu \vec{\rho}}\right) \phi_{s}
$$

where $h_{s}$ is rank-s component of $h(x, p)$. 
Proof. Represent $\phi_{s}$ as

$$
\phi_{s}=\frac{1}{2 \pi i} \oint \frac{d \lambda}{\lambda^{s+1}} \lambda^{p \partial_{p}} U_{s+\frac{d-3}{2}}\left(\frac{\mu}{\sqrt{\square}} \vec{\rho}\right) h(x, p),
$$

substitute this to (105), transfer $\lambda^{p \partial_{p}}$ to the right by using

$$
\lambda^{p \partial_{p}} U_{s+\frac{d-3}{2}}\left(\frac{\mu}{\sqrt{\square}} \vec{\rho}\right)=U_{s+\frac{d-3}{2}}\left(\frac{\mu}{\sqrt{\square}} \lambda^{-1} \vec{\rho}\right) \lambda^{p \partial_{p}}
$$

and use the identity (574) for Bessel's functions in the case $n=d-1$, to get the r.h.s equal to

$$
\frac{1}{2 \pi i} \oint \frac{d \lambda}{\lambda} e^{i \lambda^{-1}} \lambda^{p \partial_{p}} h=\sum_{s=0}^{\infty} \frac{i^{s}}{s !} h_{s}
$$

The formula (105) provides the inverse map to the "dressing". It unambiguously reconstructs the original fluctuation $h(x, p)$ in terms of "pure spin" fields $\phi_{s}$ and its $x$ derivatives. As the function $z^{s} \mathcal{C}_{s}^{\frac{d-3}{2}}\left(z^{-1}\right)$ is a polynomial in $z^{2}$ of maximal degree $2\left[\frac{s}{2}\right]$, the map (105) is well-defined in the space of power series in momenta. On the other hand, these series in derivatives terminate and every $\phi_{s}$ enters $h(x, p)$ with its $x$-derivatives only up to $s$-th order.

Furthermore, as only traceless parts of $\phi_{s}$ contributes the actions (98) at the linearized level, one can restrict $\phi_{s}$ to be traceless. Then $h(x, p)$ is traceless either, because the operator $\partial_{p}^{2}$ commutes with $\vec{\rho}$. Then the "undressing" and "reconstruction" maps (104) and (105) simplify because $\vec{\rho}$ takes the form

$$
\vec{\rho}= \pm i \partial_{x} \partial_{p}
$$

The \pm ambiguity is inessential as all expressions contain only even powers of $\rho$. In summary, for traceless "dressed" fields $h^{m(k)}, k=0,1,2, \ldots$ and "undressed" ones $\phi^{m(k)}, k=$ $0,1,2, \ldots$ the undressing (104) and reconstruction 812 maps are given by similar expressions like

$$
\begin{aligned}
& h^{m(k)}=\phi^{m(k)}+\sum_{r=1}^{\infty} c_{r} \partial_{m_{1}} \ldots \partial_{m_{2 r}} \phi^{m(k+2 r)} \\
& \phi^{m(k)}=h^{m(k)}+\sum_{r=1}^{\infty} \tilde{c}_{r} \partial_{m_{1}} \ldots \partial_{m_{2 r}} h^{m(k+2 r)}
\end{aligned}
$$

where $c_{r}, \tilde{c}_{r}$ are some real analytic functions of $\frac{\mu}{\sqrt{\square}}$.

\section{Geometry}

We begin deriving the theory which will reproduce the initial data described in the previous sections. Our guide is the notion of geometry with a source given in Introduction. 
This notion is based on a rather general reasoning, which presents nothing essentially new as compared to the bunch of habitual physical conceptions of geometry but rather provides some useful systematics which we will be using extensively. Perhaps, a slightly novel feature of our approach is the persistence in the joint consideration of what is usually called geometric structures (like tensor fields, connections etc.) on a manifold along with objects (in particular, the localized objects) that can fill an influence of those geometric structures because they determine the object's dynamics. From physical point of view such a joint analysis is natural as there is no "geo-metry" without a "meter" device.

In terms of the definitions of Introduction, the main results of the paper may be spelled as follows.

$$
\begin{aligned}
\text { test object } O= & \text { quantized point particle } \\
& \text { (with the classical action without higher time derivatives), } \\
& =\text { complex wave function of the particle } \psi(x)
\end{aligned}
$$

background field $H=$ general differential operator $\hat{H}$

$$
\begin{aligned}
& \text { coupling } S_{H}[o]=\text { action } \int d^{d} x \psi^{*} \hat{H} \psi \text { of the quantized particle } \\
& \qquad \begin{aligned}
\mathcal{G}= & \text { complexification of the algebra of } \\
& \text { quantized canonical transformations } \Rightarrow \\
\Rightarrow H= & \text { full set of conformal higher spin fields with } s=0,1,2,3, \ldots
\end{aligned}
\end{aligned}
$$

invariant induced action $\mathcal{A}[H]=\operatorname{Tr} \pi_{+}(\hat{H})=$ action of Conformal Higher Spin Theory,

where $\pi_{+}(\hat{H})$ is the projector to the subspace with positive eigenvalues of $\hat{H}$.

Below we derive the classical and the quantum geometry corresponding to the ordinary point particle in general background fields, with the classical action without higher time derivatives, and show the emergence of conformal higher spin fields as background fields of the point particle. The classical treatment is a preliminary one, while the true geometry is being uncovered in considering quantum case. 


\subsection{Classical geometry}

Consider classical $d$-dimensional point particle in general background fields. Its dynamics is set by writing down the hamiltonian action

$$
S_{H}[x(\tau), p(\tau), \lambda(\tau)]=\int_{\tau_{1}}^{\tau_{2}} d \tau\left\{p_{m} \dot{x}^{m}-\lambda H(p, q)\right\}
$$

where $x^{m}(\tau), m=0, \ldots, d-1$ are the coordinates of the particle's world line, $p_{m}(\tau)$ are the momenta and $\lambda$ is a Lagrange multiplier to the unique first class constraint $H\left(x^{m}, p_{m}\right) \approx 0$ which we shall call Hamiltonian. The Hamiltonian is supposed to be a power series in momenta,

$$
H=\sum_{k=0}^{\infty} H^{m_{1} \ldots m_{k}}(x) p_{m_{1}} \ldots p_{m_{k}}=\sum_{k=0}^{\infty} H_{k}
$$

where $H_{k}$ denotes the homogeneous polynomial of $k$-th degree in momenta.

Classical trajectories of the particle are bind to the constraint surface,

$$
H(x(\tau), p(\tau))=0
$$

The action has the local symmetry with the parameter $\nu(\tau)$, being the Hamiltonian counterpart of world line reparametrizations $x^{\prime}\left(\tau^{\prime}(\tau)\right)=x(\tau)$,

$$
\delta x^{m}(\tau)=\nu(\tau)\left\{x^{m}, H\right\}, \quad \delta p_{m}(\tau)=\nu(\tau)\left\{p_{m}, H\right\}, \quad \delta \lambda=-\dot{\nu} .
$$

This local symmetry may be fixed by implying the gauge condition

$$
\dot{\lambda}=0 \Rightarrow \ddot{\nu}=0
$$

after that only global modes of gauge transformations survive

$$
\nu(\tau)=\nu_{1} \tau+\nu_{2}
$$

These global modes may be used to set the limits of $\tau$-integration to $\tau_{1}=0, \tau_{2}=1$.

When $H_{k}=0$ for $k>2$ (i.e. when $H$ is a second order function), the model describes a particle in general gravitational + Maxwell background. It may be seen by writing down the Hamiltonian in the form

$$
\tilde{H}(x, p)=\sum_{k=0}^{2} H^{m_{1} \ldots m_{k}}(x) p_{m_{1}} \ldots p_{m_{k}}=D(x)-g^{m n}(x)\left(p_{m}-e A_{m}(x)\right)\left(p_{n}-e A_{m}(x)\right),
$$

and excluding the momenta $p_{m}$ by means of their equations of motions $\frac{\delta S}{\delta p_{m}}=0$ from the action (112), to get

$$
S=\int d \tau\left(-\frac{\dot{x}^{2}}{4 \lambda}-\lambda D+e A_{m} \dot{x}^{m}\right)
$$


and then, in the case $D \neq 0$, to exclude $\lambda$ by means of its equations of motion, to get the conventional Lagrangian action of the massive particle

$$
S=\int d \tau\left(-\sqrt{D \dot{x}^{2}}+e A_{m} \dot{x}^{m}\right)
$$

while in the case $D=0$ the action (119) describes massless particle in general gravitational +Maxwell background.

In the case Hamiltonian possesses nonvanishing components $H_{k}, k>2$, the particle experiences the influence of higher rank symmetric tensors $H^{m_{1} \ldots m_{k}}(x)$. To get an idea how this interaction looks like in the Lagrangian setup it is worth expanding general Hamiltonian around the low-spin one (118) with $D=-m^{2}, A_{m}=0$ like

$$
H(x, p)=-g^{m n}(x) p_{m} p_{n}-m^{2}-2 \bar{e} \sum_{s=0}^{\infty} h^{m_{1} \ldots m_{k}}(x) p_{m_{1}} \ldots p_{m_{k}},
$$

where $h^{m_{1} \ldots m_{k}}(x)$ is a fluctuation and $e$ is the expansion parameter, and exclude the momenta $p_{m}$ and the Lagrange multiplier $\lambda$ by means of their equations of motion in the framework of perturbations in $e$. This is done in [58], and the result is

$$
S_{H}[x(\tau)]=-\int d \tau\left\{\sqrt{-m^{2} \dot{x}^{2}}\left(1+\sum_{s=0}^{\infty} \frac{\bar{e}}{m^{2}} h_{m_{1} \ldots m_{s}}(x) \dot{x}^{m_{1}} \ldots \dot{x}^{m_{s}}\left(-\frac{m^{2}}{\dot{x}^{2}}\right)^{\frac{s}{2}}\right)+o\left(e^{2}\right)\right\} .
$$

Here each spin- $s$ contribution presents the action being equivalent to that introduced long ago in [26]. p

The action (112) is covariant w.r.t. infinite-dimensional Lie algebra $\mathcal{G}$ which has the structure of a semidirect product of all canonical transformations with parameters $\epsilon(x, p)$ to the abelian ideal of "hyper Weyl" transformations $a(x, p)$ :

$$
\begin{gathered}
S_{H+\delta H}[x+\delta x, p+\delta p, \lambda+\delta \lambda]=S_{H}[x, p, \lambda]+\text { boundary terms } \\
{\left[\delta_{\epsilon_{1}, a_{1}}, \delta_{\epsilon_{2}, a_{2}}\right]=\delta_{\left\{\epsilon_{1}, \epsilon_{2}\right\},\left\{\epsilon_{1}, a_{2}\right\}-\left\{\epsilon_{2}, a_{1}\right\}}}
\end{gathered}
$$

where

$$
\delta x^{m}=\left\{x^{m}, \epsilon(x, p)\right\}, \delta p_{m}=\left\{p_{m}, \epsilon(x, p)\right\}, \delta \lambda=-2 a(x, p) H(x, p)
$$

and

$$
\delta H(x, p)=2 a(x, p) H(x, p)+\{\epsilon, H(x, p)\} .
$$

\footnotetext{
${ }^{5}$ On our notation: we use signature $(-++\ldots+)$, alternative as compared to De Wit and Freedman's paper and re-introduce the mass parameter $m$ explicitly. The action 122 coincides with the De WitFreedman $(D W-F)$ one [26] after the identification $\varphi_{m_{1} \ldots m_{k}}^{D W-F}=-h_{m_{1} \ldots m_{k}}$ and setting $m^{2}=-1$ (the negative sign of $m^{2}$ just accounts the difference in metric's signature, $\left.\left.\sqrt{\dot{x}^{2}}\right|_{D W-F}=\left.\sqrt{-m^{2} \dot{x}^{2}}\right|_{\text {our }}\right)$.
} 
$\left(\{\right.$,$\left.\} stands for the canonical Poisson bracket, \left\{x^{m}, p_{n}\right\}=\delta_{n}^{m},\left\{x^{m}, x^{n}\right\}=\left\{p_{m}, p_{n}\right\}=0\right)$. $\epsilon$ and $a$ are supposed to have the same structure of power series in momenta as $H$ has,

$$
\begin{aligned}
& \epsilon(x, p)=\sum_{k=0}^{\infty} \epsilon^{m_{1} \ldots m_{k}}(x) p_{m_{1}} \ldots p_{m_{k}}=\sum_{k=0}^{\infty} \epsilon_{k}, \\
& a(x, p)=\sum_{k=0}^{\infty} a^{m_{1} \ldots m_{k}}(x) p_{m_{1}} \ldots p_{m_{k}}=\sum_{k=0}^{\infty} a_{k} .
\end{aligned}
$$

Then the transformations from $\mathcal{G}$ can be rewritten in terms of tensor fields being the coefficients of power series (113) and corresponding parameters $\epsilon^{m(k)}(x), a^{m(k)}(x)$. The lowest components of $\epsilon$ and $a, \epsilon_{0}, \epsilon_{1}, a_{0}$ parameterize the "low-spin" algebra of $U(1)$ "phase" transformations, $x$-diffeomorphisms and Weyl dilations, correspondingly.

According to the definitions of the previous subsection, our consideration shows that there exists the geometry with the source $O=$ "point particle", geometric structure $H(x, p)$ (113) constituting the collection of symmetric tensors of all ranks, coupling $S_{H}$ being the action of the point particle in general background fields (112), and the covariance algebra $\mathcal{G}$ (125, 126) being the semidirect product of all canonical transformations by an abelian ideal of hyper-Weyl transformations.

The set of variables $o$ is provided, in the Hamiltonian picture, by the particle's coordinates $x^{m}$, momenta $p_{m}$ and the Lagrange multiplier $\lambda$.

According to the general strategy, described in the previous subsection, one should look for an "induced" action functional for the background fields $H(x, p)$, which is invariant w.r.t. covariance transformations (126). For our purposes it is better to postpone this procedure to the next subsection, where the quantum particle is considered. Nevertheless, it is instructive to have a glimpse of what kind of theory the invariant induced action could describe. To this end one performs the linearization of the gauge transformations (126) around a particular "vacuum" configuration of background fields of the form

$$
H=-p^{2}-m^{2}=-\eta^{m n} p_{m} p_{n}-m^{2}, m^{2}=\text { Const }
$$

( $\eta^{m n}$ is the inverse Minkowski metric), which sets the dynamics of massless particle in flat Minkowski space. The linearization means introducing fluctuations $h(x, p)$ by representing general Hamiltonian as

$$
H(x, p)=-p^{2}-m^{2}+h(x, p)
$$

rewriting gauge transformations (126) in terms of the fluctuation $h(x, p)$ and keeping in gauge transformations only the terms of zero order in fluctuation. In so doing, the linearized gauge transformations are always given by the variation of the "vacuum". One 
gets

$\delta h(x, p)=\delta\left(-p^{2}-m^{2}\right)=-2 a(x, p)\left(p^{2}+m^{2}\right)-\left\{\epsilon(x, p), p^{2}\right\}=-a(x, p)\left(p^{2}+m^{2}\right)-2 p \partial_{x} \epsilon(x, p)$,

which is nothing but the gauge laws of the deformed conformal higher spin theories (83) in the case $\mu(\square)=m=$ Const. These theories were shown in Sec. (3.1) to be equivalent to ordinary conformal higher spin theories with conformal spins $s=0,1,2,3, \ldots$, formulated in terms of traceless tensors of rank $s$.

Recall the well-known fact (see also App. (A)): if an action is gauge-invariant w.r.t. full gauge transformations, then the quadratic part of its expansion around any vacuum is gauge invariant w.r.t. linearized transformation. Therefore, if one could have constructed an invariant induced action which possesses $H$ of the form (128) as a solution of the equations of motion, then the quadratic part of the action around this vacuum is a sum of conformal higher spin theories with conformal spins $s=0,1,2,3, \ldots$ described in terms of symmetric traceless tensors of rank $s$.

Note that the origin of this infinite number of traceless tensors from the Hamiltonian of the particle has a clear interpretation. Indeed, the fluctuations of the Hamiltonian are parameterized by traceless tensors as their traces are gauged away by the linearized hyper-Weyl transformations parameterized by $a(x, p)$. But the meaning of hyper-Weyl transformations is quite transparent. Namely, the classical dynamics of the particle is being developed on the constraint surface $H(x(\tau), p(\tau))=0$, and the hyper-Weylcovariance expresses the fact that the the particle fills only the shape of the constraint surface $H(x, p)=0$, not the form of the Hamiltonian as a function of phase space variables outside the constraint surface. In this sense, any two hamiltonians, which differ by multiplication by a function which never comes to zero, are equivalent. At the linearized level, fluctuations of the Hamiltonian around the vacuum $H=-p^{2}-m^{2}$ are equivalent if they differ by a function $-\left(p^{2}+m^{2}\right) a(x, p)$, so the fluctuations of the constraint surface $H=0$ are represented by the equivalence classes of functions $h(x, p)$ modulo the equivalence relation $h(x, p) \sim h(x, p)-\left(p^{2}+m^{2}\right) a(x, p)$. This equivalence does not affect only the special traceless parts of $h(x, p)$, described in App. (ब). (For $m=0$ these special traceless parts become ordinary traceless parts of $\left.h^{m(s)}, s=0,1,2,3, \ldots\right)$. Therefore, the traceless parts of the fluctuation of the Hamiltonian around the vacuum $H=-p^{2}-m^{2}$ are in one-to-one correspondence with fluctuations of the shape of the constraint surface $H(x, p)=0$.

The equivalence of linearized covariance transformations and of gauge transformations of $\mu=m$ deformed conformal higher spin theories allows to state that infinite collection of the conformal higher spin fields is in one-to-one correspondence with fluctuations of 
the shape of the constraint surface $H(x, p)=0$ around the vacuum $H=-p^{2}-m^{2}$.

Therefore, we have found a physical origin of conformal higher spin fields as background fields of the point particle. A gauge-invariant action of conformal higher spin fields, once constructed, will play a role of "induced" action (20). However, it appears to get the physically relevant results one has to quantize the particle. After all, it will be seen why the classical geometry is useful only as a motivation for, and as a limit of the full quantum treatment.

\subsection{Quantum geometry}

The covariant quantization of the model is performed easily, since there is the single first-class constraint and hence the algebra of constraints is trivial.

The particle wave functions $|\psi\rangle$ are realized in a Hermitian representation space of $d$-dimensional Heisenberg-Weyl algebra, spanned by canonical coordinates and momenta $\hat{x}^{m}, \hat{p}_{n}$, with commutation relations

$$
\left[\hat{x}^{m}, \hat{p}_{n}\right]=i \hbar \delta_{n}^{m}
$$

One may choose the coordinate representation, then $\mid \psi>$ is represented by a complex field $\psi(x)$ over $\mathcal{M}^{d},<\psi \mid$ - by a complex conjugated $\bar{\psi}(x)$ and the scalar product $<\psi_{1} \mid \psi_{2}>$ - by the integral $\int d^{n} x \bar{\psi}_{1}(x) \psi_{2}(x)$.

The covariant quantization is performed by imposing the constraint

$$
\hat{H}(\hat{x}, \hat{p}) \mid \psi>=0
$$

where some ordering of $\hat{x}$ and $\hat{p}$ is implied which is consistent with the hermiticity of the quantum Hamiltonian, $\hat{H}=\hat{H}^{\dagger}$. The role of the constraint (132) is to extract the physical states subspace . The classical analogue of this equation is the constraint equation (114). After specifying appropriate boundary conditions, this equation determines the quantum dynamics completely (for example, for hamiltonians with $H_{k}=0, k>2$, it becomes the Klein-Gordon equation in external low spin fields).

The equation (132) follows via the variational principle from the action

$$
\begin{aligned}
S[\psi] & \left.=\frac{1}{2}(<\psi|\hat{H}| \psi\rangle+(<\psi|\hat{H}| \psi>)^{*}\right)= \\
& <\psi|\hat{H}| \psi>+ \text { boundary terms. }
\end{aligned}
$$

\footnotetext{
${ }^{6}$ For general Hamiltonian, the issue of existence of a positive inner product in the physical subspace is a subtle question which we do not consider in this paper
} 
The quantum analogue of the covariance transformations (123) is the group of the most natural transformations preserving the action (133):

$$
\begin{gathered}
S_{H^{\prime}}\left[\psi^{\prime}\right]=S_{H}[\psi]+\text { boundary terms } \\
\hat{H}^{\prime}=\hat{\Omega}^{\dagger} \hat{H} \hat{\Omega} \\
\left|\psi^{\prime}>=\hat{\Omega}^{-1}\right| \psi>
\end{gathered}
$$

where $\hat{\Omega}(\hat{x}, \hat{p})$ is some invertible operator. The transformations (134,135, 136) define the quantum covariance transformations. The infinitesimal form of (135, 136) is extracted easily via the substitution $\hat{\Omega}=e^{\omega}$

$$
\begin{gathered}
\delta \hat{H}=\hat{\omega}^{\dagger} \hat{H}+\hat{H} \hat{\omega}, \\
\delta|\psi>=-\hat{\omega}| \psi>,
\end{gathered}
$$

where $\hat{\omega}(\hat{x}, \hat{p})$ is arbitrary.

Thus, one has the geometry with the source $O=$ quantized point particle; $o=$ complex scalar field, geometric structure $\hat{H}(\hat{x}, \hat{p})$, coupling $S_{H}[\psi]$ being the action of the complex scalar field $\psi(x)$, and the covariance algebra (137).

The quantum covariance transformations are interpreted as gauge transformations of the tensor fields which form the Hamiltonian as a power series in momenta. To formulate these transformations in a closed form it is useful to employ the well known "symbols of operators" technique (see e.g. [51]), which is equivalent to considering the operators $\hat{f}\left(\hat{x}^{m}, \hat{p}_{n}\right)$ as functions on the classical phase space $f\left(x^{m}, p_{n}\right)$ so that there exists (in a wide class of functions and operators) a standard invertible map $\hat{f}=Q f, f=Q^{-1} \hat{f}$. In this technique, the associative product of operators $\hat{f}_{1} \hat{f}_{2}$ becomes an associative $*$-product of functions in the phase space

$$
f_{1} * f_{2}=Q^{-1} \hat{f}_{1} \hat{f}_{2}
$$

Specifying $Q$ is said as choosing the symbol. Hereafter we choose the Weyl symbol [51]. Then the star product takes the standard "Weyl-Moyal" 51]) form

$$
\left(f_{1} * f_{2}\right)(x, p)=f_{1} \exp \left(\frac{i \hbar}{2} \stackrel{\leftrightarrow}{\nabla}\right) f_{2},
$$

where

$$
\overleftrightarrow{\nabla}=\frac{\partial}{\partial \overleftarrow{x^{n}}} \frac{\partial}{\partial \overrightarrow{p_{n}}}-\frac{\partial}{\partial \stackrel{\leftarrow}{p_{n}}} \frac{\partial}{\partial \overrightarrow{x^{n}}}
$$

This product have a structure of "semiclassical" expansion by the powers of $\hbar$. Equivalently, the star product may be written down in integral form,

$$
\begin{gathered}
\left(f_{1} * f_{2}\right)(x, p)=(\pi \hbar)^{-2 d} \int d^{d} x_{1} d^{d} p_{1} d^{d} x_{2} d^{d} p_{2} \\
\exp \left\{\frac{2 i}{\hbar}\left(\left(x-x_{2}\right) p_{1}+\left(x_{1}-x\right) p_{2}+\left(x_{2}-x_{1}\right) p\right)\right\} \\
f_{1}\left(x_{1}, p_{1}\right) f_{2}\left(x_{2}, p_{2}\right),
\end{gathered}
$$


Besides associativity, the star product possesses the standard properties of classical limit

$$
\lim _{\hbar \rightarrow 0}\left(f_{1} * f_{2}\right)(x, p)=f_{1} f_{2}, \lim _{\hbar \rightarrow 0} \frac{1}{i \hbar}\left(f_{1} * f_{2}-f_{2} * f_{1}\right)=\left\{f_{1}, f_{2}\right\}=f_{1} \nabla f_{2}
$$

In the Weyl symbol, the Hermitian conjugation

$$
f^{\dagger}=Q^{-1} \hat{f}^{\dagger}
$$

is an ordinary complex conjugation:

$$
f^{\dagger}=f^{*}
$$

Below we will nevertheless write " $f$ " instead of " $\bar{f} "$. The action of the operator with the

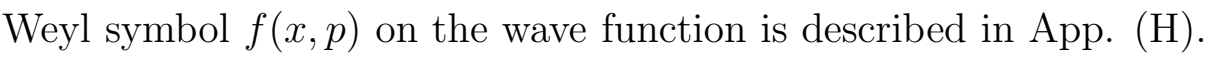

In the subsequent calculations, we use operators and their symbols on equal footing, to distinguish between two pictures we mark the operators by hat and write " * for their symbols product.

Now, we may rewrite (137) as

$$
\delta H=\omega^{\dagger} * H+H * \omega .
$$

Introducing the real and imaginary parts of $\omega$

$$
a=\operatorname{Re} \omega=\frac{1}{2}\left(\omega+\omega^{\dagger}\right), \frac{i}{\hbar} \epsilon=i \operatorname{Im} \omega=\frac{1}{2}\left(\omega-\omega^{\dagger}\right)
$$

one rewrites (137) as

$$
\delta H=[a, H]_{+}-\frac{i}{\hbar}[\epsilon, H]
$$

where [ ]+ and [] are the anticommutator and commutator w.r.t. *-product. Applying the realization of $*$ product $(140)$, one gets

$$
\delta H=2 a H+\{\epsilon, H\}+o\left(\hbar^{2}\right)
$$

In this formula one recognizes the quantum deformation of classical gauge symmetry (126). The corrections $o\left(\hbar^{2}\right)$ contain the higher derivatives of gauge parameters $a$ and $\epsilon$.

One concludes that the gauge laws (126) may acquire $o\left(\hbar^{2}\right)$ higher derivative corrections. The whole Lie algebra of infinitesimal gauge transformations (124) deforms to

$$
\begin{gathered}
{\left[\delta\left(\omega_{1}\right), \delta\left(\omega_{2}\right)\right] H=\delta\left(\omega_{3}\right) H} \\
\omega_{3}=-\left[\omega_{1}, \omega_{2}\right]
\end{gathered}
$$

which reads as follows in terms of real and imaginary parts of $\omega$ :

$$
\begin{gathered}
{\left[\delta\left(\epsilon_{1}, a_{1}\right), \delta\left(\epsilon_{2}, a_{2}\right)\right] H=\delta\left(\epsilon_{3}, a_{3}\right) H} \\
\epsilon_{3}=-\frac{i}{\hbar}\left[\epsilon_{1}, \epsilon_{2}\right]+i \hbar\left[a_{1}, a_{2}\right], a_{3}=-\frac{i}{\hbar}\left(\left[\epsilon_{1}, a_{2}\right]-\left[\epsilon_{2}, a_{1}\right]\right),
\end{gathered}
$$


$\epsilon$ parameterize the quantized canonical transformations which we shall call infinitesimal unitary transformations. In contrast with the classical transformations (124), $a$ do not form a subalgebra. These commutation relations are typical for the Lie algebras possessing Cartan decomposition, when $\epsilon$ play the role of real generators while a correspond to imaginary ones, so one may call, in analogy with $s o(p, q)$ Lie algebras, $\epsilon$ the canonical rotations and $a$ the canonical boosts. On the other hand, the whole algebra exhibits itself as a complexification of the algebra of infinitesimal unitary transformations.

Of our interest is the subalgebra of power series in momenta

$$
\omega=\sum_{k=0}^{\infty} \omega^{m_{1} \ldots m_{k}}(x) p_{m_{1}} \ldots p_{m_{k}}
$$

in the associative algebra of all phase space functions (they do form a subalgebra w.r.t. *-product (140), at least in the framework of the $\hbar$-expansion).

In the classical case, the zero-order and linear in momenta $\epsilon^{\prime} \mathrm{s}, \epsilon=\varepsilon+\xi^{m} p_{m}$, lead to $U(1)$ and general coordinate transformations. On the other hand, Weyl product has a property that the commutator of the functions $\epsilon_{1,2}$, which are linear in momenta (or independent on momenta) reduces to their Poisson bracket:

$$
\epsilon_{1,2}=\varepsilon_{1,2}(x)+\xi_{1,2}(x)^{m} p_{m} \Rightarrow\left[\epsilon_{1}, \epsilon_{2}\right]=i \hbar\left\{\epsilon_{1}, \epsilon_{2}\right\}
$$

therefore, in the quantum case one may associate the $U(1)$ and general coordinate transformations with first order $\epsilon$ 's like in the classical case, as their algebra is not deformed by the quantum corrections. At the same time, their representation in the space of phase space functions does deform,

$$
\delta H=-\frac{i}{\hbar}[\epsilon, H]=\{\epsilon, H\}+o(\hbar) .
$$

On the other hand, this deformation is a trivial one in the sense there exists change of variables from the coefficients $H^{m(s)}$ of the symbol $H(x, p)$ to ordinary tensor fields $\mathbf{H}^{m(s)}$, see Sec. (6.2) for more detail.

\subsection{Semiclassics. Generalized $W$-geometry}

In this subsection we once again make a little digression and show that the coupling $"<\psi|\hat{H}| \psi>$ " may be naturally decomposed in semiclassical series in $\hbar$ in such a way that the first, "classical" term of decomposition presents a kind of sigma-model which we call Hamilton-Jacobi sigma-model or a generalized $W$-sigma model, by the following reasons. 
Let us write the wave function $\psi(x)$ as

$$
\psi(x)=\rho(x) e^{\frac{i}{\hbar} \varphi(x)}
$$

where $\rho(x), \varphi(x)$ are arbitrary real fields and substitute it to the coupling (133). Note that an operator $\hat{H}$ with the Weyl symbol $H(x, p)=\sum_{s=0}^{\infty} H^{m(s)}(x) p_{m_{1}} \ldots p_{m_{s}}$ acts on wave functions as

$$
\hat{H} \psi(x)=\sum_{s=0}^{\infty}\left[(-i \hbar)^{s} H^{m(s)}(x) \partial_{m_{1}} \ldots \partial_{m_{s}}+\ldots\right] \psi(x),
$$

where " $+\ldots "$ denotes terms with less derivatives (for the exact expression, see (600)). It is easily seen the coupling reads

$$
S[\rho, \varphi]=\sum_{s=0}^{\infty} \int d^{d} x \rho^{2} H^{m(s)}(x) \varphi_{, m_{1}}(x) \ldots \varphi_{, m_{s}}(x)+o(\hbar)=S_{c l}+o(\hbar) .
$$

Note that the modulus of the wave function $\rho(x)$ enters the Lagrangian as an overall multiplier while the phase $\varphi$ provides derivative terms.

The variation w.r.t. $\rho$ leads to the equation of motion

$$
\frac{\delta S_{c l}}{\delta \rho(x)}=2 \rho H\left(x^{m}, \varphi, m(x)\right)=0
$$

which, if $\rho \neq 0$ is nothing but Hamilton-Jacobi equation for the action function $\varphi(x)$. The variation of (157) w.r.t. $\varphi$ leads to the equation

$$
\frac{\delta S_{c l}}{\delta \varphi(x)}=-\partial_{n}\left(\rho^{2} \frac{\partial}{\partial p_{n}} H(x, \varphi, m(x))\right)=0,
$$

which is nothing but the generalized transport equation for the density $\rho^{2}$.

This interpretation presents no surprise as the equations (158) and (159) are nothing but first terms of the semiclassical expansion of the full quantum equations of motion $\hat{H} \psi(x)=0$, while the of Hamilton-Jacobi equation and transport equation originate exactly from the substitution of the ansatz (155) to the "generalized Klein-Gordon" equation $\hat{H} \psi(x)=0$.

A less trivial fact is that the quantum covariance transformations (138) have their semiclassical counterparts, obtained by rewriting (138),

$$
\delta \psi(x)=-\hat{\omega} \psi(x)
$$

in terms of $\rho$ and $\varphi$, to get

$$
\begin{gathered}
\delta \varphi(x)=-\epsilon\left(x, \varphi_{, m}(x)\right)+o(\hbar) \\
\delta \rho^{2}(x)=-\partial_{m}\left(\rho^{2} \frac{\partial}{\partial p_{m}} \epsilon\left(x, \varphi_{, m}(x)\right)\right)-2 a\left(x, \varphi_{, m}\right) \rho^{2}(x)+o(\hbar)
\end{gathered}
$$


and taking limit $\hbar \rightarrow 0$, i.e. throwing away $o(\hbar)$ terms. The resulting transformations, parameterized by arbitrary power series in momenta $\epsilon(x, p), a(x, p)$, form exactly the same algebra as classical covariance transformations (124) and provide the covariance algebra of the Hamilton-Jacobi sigma-model (157),

$$
S_{c l H+\delta H}[\rho+\delta \rho, \varphi+\delta \varphi]=S_{c l H}[\rho, \varphi]+\text { boundary terms }
$$

The gauge transformations of the Hamiltonian are given by the classical terms of the quantum gauge transformations (137) and thereby coincide with classical gauge transformations (126).

It is notable that one has such a realization (161) of canonical transformations of $2 d-$ dimensional phase space in terms of just one scalar field $\varphi(x)$ in $d$ dimensions. This fact has many clear explanations but we do not dwell on this point here (perhaps, the simplest explanation is the derivation just delivered).

It appears the transformations of such a kind were already discussed in literature. In fact, the transformation law (161) is nothing but the basic input of $W$-geometry introduced by Hull [13]. Hull did not introduce the second field $\rho(x)$ and was looking for the coupling $S_{\tilde{F}}[\varphi]=\int d^{d} x \tilde{F}\left(x^{m}, \partial_{m} \varphi\right)$ which would possess covariance property $S_{\tilde{F}+\delta \tilde{F}}[\varphi+\delta \varphi]=S_{\tilde{F}}[\varphi]$. Here $\tilde{F}(x, p)$ called according to Hull's terminology as "cometric density" is considered as a power series in momenta whose coefficients should transform via themselves w.r.t. canonical transformations. Hull has shown the cometric density does not exist if $d>2$, while in $d=2$ the coupling exists if the canonical transformations parameters as well as coefficients of the power series $\tilde{F}(x, p)$ are subject to some constraints (while in $d=1$, the cometric density does exist). This constrained geometry is what was named " $W$-geometry" as it was shown to underly two-dimensional $W$-gravity models which gauge global $w_{\infty}$ symmetries of free massless scalar.

What we just have shown here is that if one introduces, in addition to $\varphi$, an additional field $\rho$ with transformation laws (162) then, in any dimension $d$ it is possible to construct covariant action of the form (157), and this action is nothing but the "classical limit" of the simple quadratic action " $<\psi|\hat{H}| \psi>$ " of the quantized particle. In view of the above it is natural to call the "Hamilton-Jacobi sigma-model" (157) also as "generalized $W$-sigma model".

We will return to this model in Sec. (13.2) where we will argue the action (15y) has interpretation of world volume theory of a tensionless $d-1$-brane in $d+1$ dimensions. 


\subsection{Linearization and the theorem on structure of invariant in- duced action around a flat vacuum.}

Having defined the quantum geometry relations (133)-(138) one has to look for the invariant induced action $\mathcal{A}[H]$ which is invariant w.r.t. covariance transformations (137). This is done in the next sections. But before doing this a useful information may be extracted, as in previous subsection, by studying linearization of gauge transformations around a natural vacuum

$$
\bar{H}=-\left(p^{2}+m^{2}\right),
$$

where $m^{2}$ is a real constant and $p^{2}$ is built with flat metric $g^{m n}=\eta^{m n}$. As it is recalled in App. (A), if $\bar{H}$ is a solution of the equation of motion of the action $\mathcal{A}[H]$ then the quadratic part of the action expanded around this solution is gauge invariant w.r.t. linearized gauge transformations. As in Sec. (4.1), introduce fluctuation $h(x, p)$ around the vacuum (164) as

$$
H(x, p)=\bar{H}+h(x, p),
$$

rewrite the gauge transformations (137,148) in terms of $h$ and extract the zero order in $h$, then the linearized gauge transformations are given by the gauge variation of the vacuum,

$$
\delta h=\delta \bar{H}=a * \bar{H}+\bar{H} * a-\frac{i}{\hbar}(\epsilon * \bar{H}-\bar{H} * \epsilon)=-2\left(p^{2}-\frac{\hbar^{2}}{4} \square+m^{2}\right) a(x, p)-2 p \partial_{x} \epsilon(x, p) .
$$

As compared to the analogous "classical" gauge transformations (130) of the previous

subsection, one gets the quantum correction $-\frac{\hbar^{2}}{2} \square a(x, p)$. The gauge transformations (166) are the ones of deformed conformal higher spin theories with

$$
\mu^{2}(\square)=m^{2}-\frac{\hbar^{2}}{4} \square
$$

which, as we know from the results of Sec. (3.1), are equivalent to the sum of ordinary conformal higher spin theories. More precisely, the conformal higher spin theories arise after deformed models are rewritten in terms of the "undressed" variables (98). For general $\mu=\mu(\square)$ the undressing map is nonlocal as it is manifested by the inverse square root of $\square$ in the argument of the undressing operator, this nonlocality may lead to subtleties in identifying the deformed theories with undeformed ones. It is remarkable, however, that in the case (167) with $m=0$, the undressing map is local, as in this case

$$
\mu^{2}=-\frac{\hbar^{2}}{4} \square \Rightarrow \frac{\mu}{\sqrt{\square}}= \pm \frac{i \hbar}{2}
$$

The \pm -arbitrariness is inessential as the undressing operator is an even function of it's argument.

As a result, one gets the following theorem. 
Proposition 2 If one constructs an action $\mathcal{A}[H]$ being invariant w.r.t. infinitesimal gauge transformations

$$
\delta H(x, p)=\omega^{\dagger}(x, p) * H(x, p)+H(x, p) * \omega(x, p),
$$

that possesses a vacuum of the form

$$
H=-p^{2},
$$

then the quadratic part of the perturbative expansion of $\mathcal{A}[H]$ around this vacuum is a sum of deformed conformal higher spin theories (99-(98)) with $\mu^{2}=-\frac{\hbar^{2}}{4} \square$, which is equivalent to a sum of conformal higher spin theories (50-52) with conformal spin $s=0,1,2,3, \ldots$, the equivalence is provided by local undressing and reconstruction maps (104) and (105), respectively.

It will be shown in the next sections $\mathcal{A}[H]$ exists indeed, and possesses the vacuum (170). Therefore, $\mathcal{A}[H]$ will present a consistent conformal higher spin theory action. In the subsequent treatment, we not only construct the action $\mathcal{A}[H]$ (Sec.(5)), and prove $H=-p^{2}$ is a vacuum (Sec.(7)), but explicitly calculate the quadratic action around this vacuum and get the sum of conformal higher spin theories with $s=0,1,2,3 \ldots$ (Sec.(8)).

Concluding the description of the geometry let us mention that a similar treatment of point particle in background fields has been undertaken in two-time approach [33]. In this approach, any $d$-dimensional relativistic system may be reformulated as a particular gauge of a $2+d$-dimensional one, that usually allows to highlight conformal properties of the model. On the other hand, as a consequence of two additional dimensions introduced, the analysis of dynamical content of the theory may appear to be more involved. In particular, the linearization of the equations proposed in 33 has not been shown yet to lead to some known free gauge fields in $d$-dimensions. To make contact to our treatment it is desirable to have a proposition analogous to Prop. (2), which would unambiguously indicate dynamical content of fluctuations around a vacuum. At the moment it seems unlikely that the theory proposed in [33] describes the same dynamics as our one, one of the reasons is that the action of the theory of [33] is of cubic order in dynamical fields that is not easy to relate to our non-polynomial action (215) constructed in the next section.

\section{The Action}




\subsection{Formal operator approach}

According to our strategy one has to find an action $\mathcal{A}[H]$ being gauge-invariant w.r.t. covariance transformations

$$
\delta H(x, p)=\omega^{\dagger}(x, p) * H(x, p)+H(x, p) * \omega(x, p),
$$

Below we will use what we call "the formal operator approach". This means writing all formulae in terms of operators rather than their symbols, and exploiting the standard machinery of quantum mechanics like decomposition of any operator $\hat{f}$ in terms of a full set of orthogonal states $\mid i>$ and their duals $<j \mid$ like

$$
\hat{f}=\sum_{i, j}|i><i| \hat{f}|j><j|=\sum_{i, j} f_{i j}|i><j|
$$

In fact, this formalism is equivalent to treating operators as large matrices. This formalism is very useful for elucidating the structure of the theory and for checking the algebraic properties of diverse quantities in the model. After a quantity is formulated in the formal operator approach it may be rewritten in terms of symbols. After that, one should check whether the formal properties valid for quantities built from large matrices are still valid for the quantities built from operators whose symbols are power series in momenta (113).

In terms of operators, the gauge transformations (137) read

$$
\begin{gathered}
\delta \hat{H}(x, p)=\hat{\omega}^{\dagger} \hat{H}+\hat{H} \hat{\omega} \leftrightarrow \\
\delta H_{i j}=\bar{\omega}_{k i} H_{k j}+H_{i k} \omega_{k j}
\end{gathered}
$$

Representing $\omega$ as $\omega=a+\frac{i}{\hbar} \epsilon$ one gets

$$
\delta H_{i j}=a_{i k} H_{k j}+H_{i k} a_{k j}+\frac{i}{\hbar}\left(-\epsilon_{i k} H_{k j}+H_{i k} \epsilon_{k j}\right) .
$$

$\epsilon$ parameterize infinitesimal unitary transformations, as $H$ is hermitian, it may be put into diagonal form $H_{i j}=H_{i} \delta_{i j}$, where $\delta_{i j}$ is Kronecker's $\delta$-symbol. Then transformation laws (173) are rewritten as

$$
\delta H_{i}=2 a_{i i} H_{i}
$$

In the finite form, one has

$$
H_{i}^{\prime}=e^{2 a_{i i}} H_{i}
$$

These transformations do not affect only three quantities, namely, the numbers of states 
$\mid i>$ with $H_{i}>0, H_{i}=0$ or $H_{i}<0$. Let us denote

$$
\begin{aligned}
& \sharp_{+}=\text {dimension of the subspace with } H_{i}>0 \\
& \sharp_{0}=\text { dimension of the subspace with } H_{i}=0 \\
& \sharp_{-}=\text {dimension of the subspace with } H_{i}<0 .
\end{aligned}
$$

To write down these invariants without referring to any particular basis of states one introduces three orthogonal projectors $\pi_{+}(\hat{H}), \pi_{0}(\hat{H}), \pi_{-}(\hat{H})$ to the subspaces in which $H_{i}>0, H_{i}=0$ or $H_{i}<0$, respectively,

$$
\pi_{+}+\pi_{0}+\pi_{-}=\hat{1}, \pi_{+} \pi_{0}=\pi_{+} \pi_{-}=\pi_{0} \pi_{-}=0, \pi_{+}^{2}=\pi_{+}, \pi_{0}^{2}=\pi_{0}, \pi_{-}^{2}=\pi_{-} .
$$

Then

$$
\sharp_{+}=\operatorname{Tr} \pi_{+}(\hat{H}) ; \sharp_{0}=\operatorname{Tr} \pi_{0}(\hat{H}) ; \sharp_{-}=\operatorname{Tr} \pi_{-}(\hat{H}),
$$

where trace of an operator is defined as

$$
\operatorname{Tr} \hat{f}=\sum_{i} f_{i i}
$$

Thus, the only functional being invariant w.r.t. gauge transformations (176) is

$$
\mathcal{A}[\hat{H}]=\alpha_{+} \sharp_{+}[\hat{H}]+\alpha_{0} \sharp_{0}[\hat{H}]+\alpha_{-} \sharp-[\hat{H}],
$$

where $\alpha_{+}, \alpha_{0}, \alpha_{-}$are some constants. As the sum of all dimensions $\sharp_{+}+\sharp_{0}+\sharp_{-}$does not depend on $H$, only two constants are independent.

The projectors $\pi_{+}, \pi_{0}, \pi_{-}$may be formally written down if one uses the notion of a function of operator. Given a function of a real variable $F(\sigma)$ and the decomposition of the Hilbert space into a direct sum of eigenspaces $\mid H_{i}>$ of a hermitian operator $\hat{H}$,

$$
\hat{H}\left|H_{i}>=H_{i}\right| H_{i}>
$$

the function of operator $\hat{H}$ is defined as the operator which acts in each eigenspace as

$$
F(\hat{H})\left|H_{i}>=F\left(H_{i}\right)\right| H_{i}>.
$$

Let us define three functions of a real variable $\sigma$ by the rule

$$
\begin{gathered}
t_{+}(\sigma)=0, s \leq 0, t_{+}(\sigma)=1, s>0 \\
t_{0}(\sigma)=0, s \neq 0, t_{0}(\sigma)=1, s=0 \\
t_{-}(\sigma)=0, s \geq 0, t_{-}(\sigma)=1, s<0 \\
t_{+}+t_{0}+t_{-}=1, t_{+}^{2}=t_{+}, t_{0}^{2}=t_{0}, t_{-}^{2}=t_{-} ; t_{+} t_{0}=t_{+} t_{-}=t_{-} t_{0}=0
\end{gathered}
$$


Then

$$
\pi_{+}=t_{+}(\hat{H}), \pi_{0}=t_{0}(\hat{H}), \pi_{-}=t_{-}(\hat{H}),
$$

and general invariant functional (181) takes the form

$$
\mathcal{A}[H]=\operatorname{Tr}\left(\alpha_{+} t_{+}[\hat{H}]+\alpha_{0} t_{0}[\hat{H}]+\alpha_{-} t_{-}[\hat{H}]\right) .
$$

As $t_{+}+t_{0}+t_{-}=1$ the action is rewritten as

$$
\mathcal{A}[H]=\operatorname{Tr}\left(\left(\alpha_{+}-\alpha_{-}\right) t_{+}[\hat{H}]+\left(\alpha_{0}-\alpha_{-}\right) t_{0}[\hat{H}]+\alpha_{-}\right),
$$

that is, up to an infinite constant $\operatorname{Tr} \alpha_{-}$is

$$
\mathcal{A}[H]=\operatorname{Tr}\left(\gamma_{+} t_{+}[\hat{H}]+\gamma_{0} t_{0}[\hat{H}]\right)
$$

To actually calculate this expression for general $H(x, p)$ one has to rewrite the last expression in the langauge of symbols. This is done in the next two subsections for the case $\gamma_{+}=1, \gamma_{0}=\frac{1}{2}$, while generalization to arbitrary $\gamma$ 's does not produce new results.

\subsection{General class of functionals $\operatorname{Tr} \mathbf{F}\left(\mathrm{H}^{*}\right)$. Semiclassical expansion as a low-energy expansion.}

First of all we show how to rewrite general functional of the form

$$
A_{F}[H]=\operatorname{Tr} F(\hat{H})
$$

in terms of symbols. Let $\hat{H}$ be an operator, which acts on the wave functions by the rule

$$
(\hat{H} \psi)(x)=\int d^{d} x^{\prime} \tilde{H}\left(x, x^{\prime}\right) \psi\left(x^{\prime}\right),
$$

with the integral kernel $\tilde{H}\left(x, x^{\prime}\right)$. The trace of the operator is defined as

$$
\operatorname{Tr} \hat{H}=\int d^{d} x \tilde{H}(x, x),
$$

and for a wide class of operators this definition coincides with (180).

On the other hand, if $H(x, p)$ is the Weyl symbol of $\hat{H}$, connected with the kernel by the formula (598) of Appendix ([H), the same expression is identically rewritten as

$$
\operatorname{Tr} \hat{H}=(2 \pi \hbar)^{-d} \int d^{d} x d^{d} p H(x, p)
$$

In the symbol picture, the notion of function of operator is transformed into the notion of $*$-function. Given an ordinary function $F(\sigma)$ and a function $H(x, p)$, one defines $*$ functions of $H$ by the following rules [48]. *-polynomials are defined as

$$
(H *)^{k}=H * \ldots * H \quad(\mathrm{k} \text { times }) .
$$


Then, if a function is decomposed in Tailor series, $*$-function is defined by the same Tailor series with the (polynomials $\leftrightarrow *$-polynomials) substitution. This way one can define $*$-exponent:

$$
\exp (\lambda H *)=\sum_{k=0}^{\infty} \frac{1}{k !}(\lambda H *)^{k}, \lambda \in C .
$$

Note that in contrast to ordinary exponent series which converges for arbitrary arguments, the convergence of $*$-exponent is an issue. Then, let

$$
F(\sigma)=\int_{C} d \lambda \tilde{F}(\lambda) e^{\lambda \sigma}
$$

where the integration is performed along a contour $C$ in a complex plane, be some FourierLaplace type representation for $F(\sigma)$. Then $F(H *)$ is defined as

$$
F(H *)=\int_{C} d \lambda \tilde{F}(\lambda) \exp (\lambda H *)
$$

For a wide class of symbols and operators the symbol of the function of operator $F(\hat{H})$ is given by the $*$-function $F(H *)$, and the expression (189) is mapped to

$$
A_{F}[H]=\operatorname{Tr} F(H *)=(2 \pi \hbar)^{-d} \int d^{d} x d^{d} p F(H *)
$$

Now discuss the invariance properties of the trace operation. In the formal operator formalism, $\operatorname{Tr}(F(\hat{H}))$ is invariant w.r.t. infinitesimal unitary transformations $\delta \hat{H}=$ $-\frac{i}{\hbar}(\hat{\epsilon} \hat{H}-\hat{H} \hat{\epsilon})$ as every eigenvalue $H_{i}$ is invariant. In the symbol formalism, this invariance is controlled by the property

$$
(A * B-B * A)=(\text { total derivative }),
$$

and therefore (needless to say, w.r.t. infinitesimal unitary transformations $F(H *)$ transforms by the same rule as $\left.H, \delta F(H *)=-\frac{i}{\hbar}[\epsilon, F(H *)]\right)$

$$
\delta \operatorname{Tr} F(H *)=-\frac{i}{\hbar} \int d^{d} x d^{d} p[\epsilon, F(H *)]=\int d^{d} x d^{d} p \text { (total derivative) }
$$

and equals zero if total derivative vanishes at infinity (we suppose there are no bursts in the total derivative so integral of the total derivative resides at infinity, it will always be the case for the expressions we deal with in the paper). In our treatment, the vanishing of the total derivatives at infinity will take place due to reasons to be discussed a little bit later (see discussion after (230)).

Note also the "simple multiplication" property of the Weyl symbol

$$
\operatorname{Tr}(A * B)=\operatorname{Tr}(A B)+\int d^{d} x d^{d} p \text { (total derivative) }
$$

useful in diverse calculations. 
Our main tool for calculating expressions like (197) is as follows.

Every functional (197) may be rewritten in a standard field theoretic form providing a kind of low-energy expansion, like the $\alpha^{\prime}$ expansion in string theory where, if one neglects the overall $\frac{1}{(2 \pi \hbar)^{d}}$ prefactor, $\hbar^{2}$ plays the same role of parameter counting the number of space-time derivatives as $\alpha^{\prime}$ does. The $\hbar^{0}$-term contains no space-time derivatives and exhibits itself as a cosmological term. Indeed, every $*$-function $F(H *)$ turns out to possess a semiclassical expansion in powers of $\hbar$

$$
F(H *)=\sum_{k=0}^{\infty} \hbar^{2 k} F_{2 k}(H), F_{0}(H)=F(H)
$$

To ground this statement, consider $*$-exponent (194)

$$
u(\lambda H \mid \hbar) \equiv \exp (\lambda H *)
$$

It satisfies the differential equation

$$
\frac{\partial}{\partial \lambda} u(\lambda H \mid \hbar)=H * u(\lambda H \mid \hbar)=H \exp \left(\frac{i \hbar}{2} \overleftrightarrow{\nabla}\right) u(\lambda H \mid \hbar)
$$

Writing down $u(\lambda H \mid \hbar)$ in a form of the semiclassical expansion

$$
u(\lambda H \mid \hbar)=\sum_{k=0}^{\infty}\left(\frac{i \hbar}{2}\right)^{k} u_{k}(\lambda H)
$$

one gets the simple recurrent differential equations for $u_{k}(\lambda H)$ :

$$
\begin{gathered}
\frac{\partial}{\partial \lambda} u_{0}=H u_{0} \\
\frac{\partial}{\partial \lambda} u_{1}=H \stackrel{\leftrightarrow}{\nabla} u_{0}+H u_{1} \\
\frac{\partial}{\partial \lambda} u_{2}=\frac{1}{2} H(\stackrel{\leftrightarrow}{\nabla})^{2} u_{0}+H \stackrel{\leftrightarrow}{\nabla} u_{1}+H u_{2} \\
\ldots \\
\frac{\partial}{\partial \lambda} u_{k}=\sum_{l=0}^{k} \frac{1}{l !} H(\overleftrightarrow{\nabla})^{l} u_{k-l}
\end{gathered}
$$


etc., wherefrom accounting the initial condition $u(0)=1$ one gets the solution]

$$
\begin{gathered}
u_{0}=e^{\lambda H} \\
u_{1}=e^{\lambda H} \frac{\lambda^{2}}{2}(H \overleftrightarrow{\nabla} H)=0 \\
\left.u_{2}=e^{\lambda H}\left(\frac{\lambda^{2}}{4}\left(H \overleftrightarrow{\nabla}^{2} H\right)-\frac{\lambda^{3}}{6}(H \stackrel{\leftrightarrow}{\nabla} H \overleftrightarrow{\nabla} H)\right)\right) \\
\ldots \\
u_{2 k+1}=0, \quad u_{2 k}=e^{\lambda H} v_{2 k}\left(\lambda H, \lambda \partial H, \ldots, \lambda(\partial)^{2 k} H\right)
\end{gathered}
$$

where $v_{2 k}$ is a polynomial of the highest degree $3 k$ in $\lambda H$ and its derivatives w.r.t. phase space variables $(x, p)$. The total order of derivatives in each $\hbar^{k}$ term equals $2 k$. Note that in the Weyl symbol (141) $H \stackrel{\leftrightarrow}{\nabla} H=0$, therefore the terms including this combination vanish.

Substituting the decomposition (203-206) in a Fourier-Laplace representation (196) one gets the semiclassical decomposition for an arbitrary $*$-function

$$
\begin{gathered}
F(H *)=F(H)+ \\
-\frac{\hbar^{2}}{4}\left(\frac{1}{4} F^{\prime \prime}(H)\left(H \overleftrightarrow{\nabla}^{2} H\right)-\frac{1}{6} F^{\prime \prime \prime}(H)(H \stackrel{\leftrightarrow}{\nabla} H \overleftrightarrow{\nabla} H)\right)+ \\
+\ldots+\left(\frac{\hbar}{2}\right)^{2 k} \sum_{n=2}^{3 k} F^{(n)}(H) \operatorname{Pol}_{2 k, n}\left(H, \partial H, \ldots \partial^{4 k} H\right)+\ldots
\end{gathered}
$$

where $F^{\prime \prime}, F^{\prime \prime \prime}, \ldots, F^{k}$ are the second, third and highest derivatives of $F$ by it's argument, $\operatorname{Pol}_{2 k, n}$ are some monomials of degree $n$ in $H$ and its derivatives w.r.t. phase space variables $(x, p)$ up to the order $4 k$.

It is clear from the structure of $*$ product and $\stackrel{\leftrightarrow}{\nabla}$ operator that the numbers of $x$ and $p$-derivatives in each term are equal to each other and equal $2 k$. Thus, every $\hbar^{2 k}$-order of the semiclassical expansion $F(H *)$ is nothing but the $2 k$-th order in $x$ derivatives. Therefore, the semiclassical expansion takes a form of low energy expansion for $F(H *)$ where $\hbar$ is an expansion parameter counting number of $x$-derivatives. Calculating the trace (197), one finds the low-energy decomposition of the action

$$
A_{F}[H]=A_{0}+\hbar^{2} A_{2}+\hbar^{4} A_{4}+\ldots=(2 \pi \hbar)^{-d} \int d^{d} x d^{d} p F(H(x, p))+\hbar^{2} A_{2}+\hbar^{4} A_{4}+\ldots
$$

\footnotetext{
${ }^{7}$ The expressions like $(A \stackrel{\leftrightarrow}{\nabla} B \stackrel{\leftrightarrow}{\nabla} C)$ are to be understood in a sense that $\stackrel{\leftrightarrow}{\nabla}$ acts only on its neighbors, i.e. the left $\stackrel{\nabla}{\nabla}$ acts on $A$ and $B$ while the right one acts on $B$ and $C$.
} 
The classical term $A_{0}$ is invariant w.r.t. classical canonical transformations, $\delta H=\{\epsilon, H\}$ up to a total derivative. Here it represents the "cosmological term". Therefore, the cosmological term is intimately related to the classical limit of the quantized particle geometryl?

To make contact with standard field theoretic actions one has to integrate in (208) over momenta, then one gets the standard actions for the infinite collection of symmetric tensor fields on the $x$-space.

There do exist such "Lagrangians" $F$ that the integration over momenta is well-defined in the class of power series in momenta (113), at least within the framework of some perturbative procedure. At least with our choice for $F$ (see the next section, Eq. (211)), all integrations over $p_{m}$ are finite and easily calculated at any order in $\hbar$.

\subsection{The Action}

Among general functionals of the form (197), there exists the unique one, where the gauge invariance $\delta H=-\frac{i}{\hbar}[H, \epsilon]$ is extended to the full algebra of similarity transformations of the form

$$
\delta H=\omega^{\dagger} * H+H * \omega
$$

which is nothing but the covariance algebra of our geometry. Gauge parameters $\omega$ are required to have the form of power series in momenta, with coefficients being tensor fields with compact support in $x$-space. Note that this restriction defines a subalgebra of the total algebra of smooth phase-space functions (152), at least perturbatively in $\hbar$.

We recall that in the trace operation the order of product factors is inessential up to a total derivative, then the variation of $A_{F}[H]$ under (209)

$$
\delta A_{F}[H]=2 \int d^{d} x d^{d} p F^{\prime}(H *) * H * a+\text { total derivative. }
$$

The only solution providing vanishing of this variation (modulo total derivative) for arbitrary $H$ and arbitrary $a$ is

$$
F(H)=\nu_{1} \theta\left(\nu_{2} \sigma\right)+\nu_{3} ; F^{\prime}(\sigma)=\nu_{1} \nu_{2} \delta\left(\nu_{2} \sigma\right)
$$

here $\nu_{1,2,3}$ are real constants, which may be set, without loss of generality, to $\nu_{1}=\nu_{2}=$

8 This, very natural, appearance of the cosmological term conforms with Fradkin - Vasiliev results on the inevitable appearance of cosmological backgrounds in higher spin models 3 . 
$1, \nu_{3}=0$, and $\theta(\sigma)$ is the "step" $\theta$-function:

$$
\begin{aligned}
& \theta(\sigma)=0, \quad \sigma<0 \\
& \theta(\sigma)=\frac{1}{2}, \quad \sigma=0 \\
& \theta(\sigma)=1, \quad \sigma>0,
\end{aligned}
$$

while $\delta(\sigma)=\frac{\partial}{\partial \sigma} \theta(\sigma)$ is an ordinary $\delta$-function. The variation vanishes due to the identity

$$
\delta(H) * H \equiv 0
$$

This identity is not a formal one, in fact being decomposed in semiclassical series like (207) it generates an infinite number of relations expressed in terms of derivatives of the equation $\delta(\sigma) \sigma \equiv 0$ and valid as identities for distributions.

The choice of $F(H)$ we just have made conforms with considerations based on the formal operator approach of subsec. (5.1). and corresponds to the option

$$
\gamma_{+}=1, \gamma_{0}=\frac{1}{2}
$$

Thus, the invariance w.r.t. covariance transformations (135), (148) fixes the form of action unambiguously, up to the choice of the constant $\theta(0)$ which, as it will be seen below, is inessential so our choice (214) does not spoil generality. Thus,

$$
\mathcal{A}[H]=\operatorname{Tr} \theta(H *) .
$$

The action can be formally represented as an "invariant induced action"

$$
\mathcal{A}[H]=\int \mathcal{D} x \mathcal{D} p \frac{\mathcal{D} \lambda}{\lambda} \delta(\dot{\lambda}) \exp \left\{\frac{i}{\hbar} \int_{0}^{1} d \tau(p \dot{x}-\lambda H(x, p))\right\},
$$

where the $\delta(\dot{\lambda})$-insertion implies the natural gauge $\lambda=$ const (116), and the remaining global $\tau$-reparametrizations (117) are used to set the limits of $\tau$-integration to 0 and 1 . After this gauge is implied the functional integral by $\lambda$ reduces to an ordinary one by $\lambda$ 's zero mode. Choosing a special integration contour leads to our action (215). Indeed, recalling the functional integral representation of the trace of the evolution operator [51]

$$
\operatorname{Tr} \exp ^{i \lambda \hat{H}}=\int \mathcal{D} x \mathcal{D} p \exp \left\{\frac{i}{\hbar} \int_{0}^{\lambda} d \tau(p \dot{x}-H(x, p))\right\},
$$

and the integral representation for $\theta$-function

$$
\theta(\sigma)=\frac{1}{2 \pi i} \int_{-\infty}^{\infty} \frac{d \omega}{\omega-i \varepsilon} e^{i \omega \sigma}
$$


one gets (215) after specifying integration contour in $\lambda$-space as

$$
C_{\theta}: \lambda(\tau)=\tau-i \varepsilon ; \tau \in(-\infty, \infty), \varepsilon \rightarrow 0^{+}
$$

Note that this is not the contour that provides the one-loop effective action $\operatorname{Tr} \operatorname{Ln}(\hat{H})$, the latter would arise after choosing the contour

$$
C_{\text {one-loop }}: \lambda(\tau)=\tau, \tau \in[\varepsilon, \infty), \varepsilon \rightarrow 0^{+}
$$

which is ultraviolet divergent in general as $\varepsilon$ tends to zero.

Thus, the action has the form

$$
\begin{gathered}
\mathcal{A}[H]=\operatorname{Tr} \theta(H *)=(2 \pi \hbar)^{-d} \int d^{d} x d^{d} p\{\theta(H)+ \\
-\frac{\hbar^{2}}{4}\left(\frac{1}{4} \delta^{\prime}(H)\left(H \leftrightarrow^{2} H\right)-\frac{1}{6} \delta^{\prime \prime}(H)(H \stackrel{\leftrightarrow}{\nabla} H \stackrel{\leftrightarrow}{\nabla} H)\right)+ \\
\left.+\ldots+\left(\frac{\hbar}{2}\right)^{2 k} \sum_{n=2}^{3 k} \delta^{(n-1)}(H) \operatorname{Pol}_{2 k, n}\left(H, \partial H, \ldots \partial^{l} H\right)+\ldots\right\}
\end{gathered}
$$

Below, we will refer the $(2 \pi \hbar)^{-d} \hbar^{2 k}$ - terms as $\hbar^{2 k}$-ones, in so doing $2 k$ appear to be equal to the total number of $x$-derivatives of dynamical fields.

\subsection{Classes of hamiltonians. $\mathcal{C}, \mathcal{C}_{p}$ and $\tilde{\mathcal{C}}_{p}$}

It is important to find a reasonable class of hamiltonians $H(x, p)$ for which the action is well-defined. One example of such a class, which we denote $\mathcal{C}$ is provided by hamiltonians for which the constraint surface $H(x, p)=0$ is a boundary of a compact "inside" domain In in the phase space

$$
\{\operatorname{In} \mid(x, p) \in \operatorname{In} \Leftrightarrow H(x, p)<0\},\{\mathcal{C} \mid H \in \mathcal{C} \Leftrightarrow \text { In is compact }\}
$$

Note that this class of hamiltonians is invariant, at least perturbatively in $\hbar$, w.r.t. gauge transformations (209). 9

To show $H \in \mathcal{C}$ is a good option for the action (215), consider first the classical, $\hbar^{0}$ term, proportional to $\theta(H)$. The constraint surface $H=0$ is a boundary of two domains In and Out in the phase space, such that the phase space is split into two regions: In "inside", with $H>0$, and Out "outside", with $H<0$. As $\theta(H)=1$ "inside" and $\theta(H)=0$

\footnotetext{
${ }^{9}$ As $H=0$ is a compact surface, for general $H \in \mathcal{C}$ this is equivalent to requiring that the surface $H=E$ is also compact, for $E$ being small enough. This implies that $\hat{H} \in \mathcal{C}$ possesses a discrete spectrum which starts from $E=0$.
} 
"outside", the classical term equals (up to the multiplier) the volume of In. Note that the value $\theta(0)$ is inessential as the surface $H=0$ has zero measure in the phase space.

The higher $\hbar^{2 k}$-terms, interpreted as quantum corrections to the classical term, are proportional to derivatives of $\delta$-function. Therefore, they are also finite as due to the presence of $\delta^{(k)}(H)$ the integrals reside on the compact constraint surface $H=0$.

Thus, one has the following statement.

Proposition 3 In the case constraint surface $H \in \mathcal{C}$, i.e. if the constraint surface $H(x, p)=0$ is a boundary of a compact domain, the full action is the "quantized volume" of the "inside" region $H(x, p)>0$ in the phase space. After the expansion into the semiclassical series (221), the classical term is the volume of the "inside" region. The quantum corrections of order $\hbar^{2 k}, k=1,2, \ldots$ are given by integrals of densities localized on the constraint surface $H(x, p)=0$.

So far we have described the case when $H \in \mathcal{C}$ and the action is finite (at least semiclassically, i.e. in each order in $\hbar)$. Note this case is not precisely what we need. Actually, we need the situation when 1) $H(x, p)$ is a power series in momenta with coefficients $H^{m(k)}(x)$ being arbitrary smooth tensor fields in $x$-space 2) the result of integration by momenta in (215) is finite, while the action need not be finite. In this case, the action is interpreted as a conventional one represented by an integral by $x$ of a Lagrangian being a function of tensor fields $H^{m(k)}(x)$ and their $x$-derivatives. Therefore, it is vital to find a reasonable class of $H(x, p)$ for which the integrals by momenta converge and can be calculated explicitly. A good option is the analog of the class $\mathcal{C}$ described above, the class $\mathcal{C}_{p}$ of hamiltonians $H(x, p)$ for which the constraint surface $H(x, p)=0$ is, for each $x$, a boundary of a compact "inside" domain $\left\{\operatorname{In}_{x} \mid p \in I n_{x}\right.$ if $\left.H(x, p)>0\right\}$.

Indeed, then already before $x$-integration each term of the semiclassical expansion (208) is an integral of a regular expression by $I n_{x}$ (classical term) or by the constraint surface (quantum corrections). As $I n_{x}$ and $H=0$ are compact each term of the $\hbar$-expansion is well-defined.

Thus, provided $H \in \mathcal{C}_{p}$ the action (215) has a form of an $x$-integral of the Lagrangian, depending on components of $H(x, p)$ and their $x$-derivatives.

The first, classical term of the semiclassical expansion of the Lagrangian, corresponding to the action (215) has the interpretation of the volume of the domain bounded by the constraint surface. This is clear due to the same reasoning as for the $H \in \mathcal{C}$ calculations. Namely, $\theta(H)=1$ "inside" and $\theta(H)=0$ "outside", therefore, the classical term in the Lagrangian equals (up to the multiplier $(2 \pi \hbar)^{-d}$ ) the volume of $I n_{x}$. This classical term does not contain $x$-derivatives of the components of $H$ and is interpreted as a cosmological 
term. Again the value $\theta(0)$ is inessential as the surface $H=0$ has zero measure in the phase space.

The higher $\hbar^{2 k}$-terms, proportional to derivatives of $\delta$-function, contain $2 k$ space-time derivatives of the dynamical fields and are interpreted as quantum corrections to the classical(=cosmological) term. Thus the Lagrangian of the theory may be spelled as follows.

Proposition 4 In the case constraint surface $H \in \mathcal{C}_{p}$, i.e. if for each $x$ the constraint surface $H(x, p)=0$ is a boundary of a compact domain, the full Lagrangian is the "quantized volume" of the "inside" region $H(x, p)>0$ in the phase space. After the expansion into the semiclassical series (221), the classical term is the volume of the "inside" region, it does not contain $x$-derivatives of the dynamical fields and therefore plays the role of a cosmological term. The quantum corrections of order $\hbar^{2 k}, k=1,2, \ldots$ contain $x$-derivatives of the dynamical fields of order $2 k$ and are given by integrals of densities localized on the constraint surface $H(x, p)=0$.

Note that of our main interest in this paper are the hamiltonians $H(x, p)$ which are the power series in momenta (113). Therefore, we are interested if it is possible to have an overlap between $\mathcal{C}_{p}$ and the space of power series in momenta. It is clear that these two classes have wide overlap which we denote $\tilde{\mathcal{C}}_{p}$. In order a power series in momenta $H(x, p)$ to belong to $\mathcal{C}_{p}$ the coefficients of this power series should satisfy some inequalities which do not impose algebraic constraints on the coefficients $H^{m(k)}$ of $H(x, p)$ and therefore do not reduce the dimension of the space of power series. We refer these inequalities as $\mathcal{C}_{p}$-restrictions. Although we do not find these restrictions explicitly we suppose they are satisfied so that

$$
H(x, p)=\sum_{s=0}^{\infty} H^{m_{1} \ldots m_{s}}(x) p_{m_{1}} \ldots p_{m_{s}} \in \mathcal{C}_{p}
$$

A good test of the validity of the last formula would be the convergence of all integrals by momenta in all calculations we are going to perform, while the appearance of divergencies would be interpreted as spoiling the $\mathcal{C}_{p}$-restrictions.

For practical calculations, it is convenient considering general hamiltonians as a sum of the "low spin" and "higher spin" parts,

$$
\begin{gathered}
H(x, p)=H_{2}(x, p)+w \Delta H(x, p) \\
H_{2}(x, p)=\sum_{s=0}^{2} H^{m_{1} \ldots m_{s}}(x) p_{m_{1} \ldots p_{m_{s}}}=D(x)-g^{m n}(x) \Pi_{m} \Pi_{n}, \Pi_{m}=p_{m}-A_{m}(x) \\
\Delta H(x, p)=\sum_{s=3}^{\infty} H^{m_{1} \ldots m_{s}}(x) p_{m_{1}} \ldots p_{m_{s}},
\end{gathered}
$$


where $D(x) \geq 0$, the metric $g_{m n}$ is Euclidean. Then, if $\Delta H=0$ then $H \in \mathcal{C}_{p}$ as, for each $x$, the constraint surface $H=H_{2}=0$ is a generalized sphere of radius $\sqrt{D \operatorname{det}(g)}$. If to treat general $H$ of the form (224) perturbatively by considering $\Delta H$ as a fluctuation with the expansion parameter $w$, then all the expressions in (221) are to be calculated perturbatively in powers of $w$. Notably, given a fixed order of the semiclassical expansion (221), each term of its $w$-expansion is finite, because it is represented by integrals of the form $\delta^{(k)}\left(H_{2}\right) L\left(H_{2}, \Delta H, \partial H_{2}, \partial \Delta H, \ldots\right)$ localized on the generalized sphere $H_{2}=0$. Thus, the Lagrangian is finite in the framework of the perturbative expansion in $\hbar$ and $w$ in the sense each term of this expansion is finite for arbitrary hamiltonians of the form (224), even if $\mathcal{C}_{p}$-restrictions are not obeyed. In the case $H \in \mathcal{C}_{p}$, the series in $w$ converge to exact expressions written down in terms of entire $H$ without its decomposition into low spin and higher spin parts. In general, summing the power series in $w$ may lead to divergencies, we interpret these divergencies as arising because $H(x, p)$ ceases to belong to $\mathcal{C}_{p}$.

An important remark concerns the difference between even- and odd- dimensional case. All the integrals to be calculated are given by successive derivatives by $D$ of the expressions like $\int d^{d} p \theta\left(D-p^{2}\right) p_{m_{1}} \ldots p_{m_{2 s}} \sim D^{\frac{d}{2}+s} \sqrt{g}\left(g_{m(2)}\right)^{s}$. Therefore, in even dimensions all expressions contain only non-negative powers of $D$ while in odd ones arbitrary negative powers of $D$ are possible. One more point is that in even dimensions one has a huge variety of $D^{0}$-terms, i.e. there exists a well-defined and nontrivial limit $D \rightarrow 0$.

After integrals by momenta are evaluated either in terms of $w$-expansion for $H$ of the form (224) or explicitly for $H \in \mathcal{C}_{p}$ one gets the Lagrangian that depends on the tensor fields $H^{m(k)}$ and their $x$-derivatives. Then one takes away the $\mathcal{C}_{p}$-restrictions on the coefficients of the power series and considers $H^{m(s)}$ of a general form. The Lagrangian remains finite as in terms of $H^{m(s)}$ it is represented by the same expression. Thus one obtains the analytic continuation of the Lagrangian to the cases when it does not have the interpretation of the "quantized volume". This includes the cases when the metric in (224) is not Euclidean, since the surface $H_{2}=0$ is clearly non-compact in this case. To illustrate what happens we deliver a simple example. Consider the integrals

$$
\begin{gathered}
I_{1}\left[A_{0}\right]=\int d^{d} p \delta\left(m^{2}-\eta^{m n} p_{m} p_{n}\right) A_{0}(p) \\
I_{2}\left[A_{1}, A_{2}\right]=\int d^{d} p \delta\left(m^{2}-\eta^{m n} p_{m} p_{n}\right) A_{1}(p) A_{2}(p),
\end{gathered}
$$

where $A_{0,1,2}(p)$ are power series in momenta,

$$
A_{0,1,2}(p)=\sum_{s=0}^{\infty} A_{0,1,2}^{m_{1} \ldots m_{s}} p_{m_{1}} \ldots p_{m_{s}}
$$

and the metric $\eta^{m n}$ is Euclidean. The integration runs over $d-1$-sphere of radius $m$ and 
are clearly well-defined. The integrals are

$$
\begin{gathered}
\left.I_{[} A_{0}\right]=\sqrt{\operatorname{det} \eta_{m n}} \sum_{s=0}^{\infty} b_{s} m^{d-1+2 s} A^{m_{1} \ldots m_{2 s}} \eta_{m_{1} m_{2}} \ldots \eta_{m_{2 s-1} m_{2 s}} \\
I\left[A_{1}, A_{2}\right]=\sqrt{\operatorname{det} \eta_{m n}} \sum_{s=0}^{\infty} c_{s} m^{d-1+2 s} A_{1}^{m_{1} \ldots m_{s}} A_{2} m_{1} \ldots m_{s}
\end{gathered}
$$

where $b_{s}, c_{s}$ are some easily calculable numbers, and the contractions are by the Euclidean metric $\eta^{m n}$. The analytic continuation of these expression is achieved just by ascribing another signature to the metric, after this continuation, the expression $I[A], I\left[A_{1}, A_{2}\right]$ are still well-defined but become pure imaginary, as it is standard in passing from Euclidean to Minkowski space. The imaginary unit pre-factor is to be absorbed by the same pre-factor which comes from the analytic continuation of the integration measure in $x$-space.

Contrary, if one tries to change signature of the metric before integration by momenta in (221) one gets divergent expression as the surface $p^{2}=m^{2}$ is not a sphere but a noncompact hyperboloid or a cone. To summarize, after integration by momenta and analytic continuation to the cases when $H \notin \mathcal{C}_{p}$ the Lagrangian is still well-defined but lacks the interpretation of the "quantized volume".

Note that the analytic continuation of the quadratic Hamiltonian $H_{2} \in \mathcal{C}_{p}$ (224) to Minkowski space, achieved by changing the sign of $g^{00}(x)$, lead to the Hamiltonian of tachyon. Clearly, the Minkowski version of all $H_{2} \in \mathcal{C}_{p}$ in the framework of the wexpansion (224) lead to hamiltonians that describe tachyon in low and higher spin fields. Thus one can name the backgrounds $H \in \mathcal{C}_{p}$ analytically continued to Minkowski space as tachyon backgrounds.

\subsection{Actual gauge invariance of the action}

Now let us discuss the gauge invariance of the action (215, 221). First of all, by construction it is "formally invariant", that means its variation w.r.t. gauge transformations (209) is the integral of a total derivative. It appears this formal invariance may be proved to be an actual gauge invariance, if $H \in \mathcal{C}_{p}$, at least in the framework of the semiclassical expansion by powers of $\hbar$.

Instead of finding this total derivative explicitly (although it may be done after some algebra) we note that as the action (221) and gauge transformations (209) are expanded in power series in $\hbar$

$$
\mathcal{A}=\mathcal{A}_{0}+\hbar^{2} \mathcal{A}_{2}+\ldots, \delta H=\delta_{0} H+\hbar \delta_{1} H+\hbar^{2} \delta_{2} H+\ldots
$$


the conditions of gauge invariance of the action may be expanded in powers of $\hbar$ either, $\delta \mathcal{A}=\delta_{0} \mathcal{A}_{0}+\hbar\left(\delta_{1} \mathcal{A}_{0}\right)+\hbar^{2}\left(\delta_{o} \mathcal{A}_{1}+\delta_{2} \mathcal{A}_{0}\right)+\ldots=\int d^{d} x d^{d} p\left\{[\text { t.d. }]_{0}+\hbar[\text { t.d. }]_{1}+\hbar^{2}[\text { t.d. }]_{2}+\ldots\right\}$

where $\delta_{k} \mathcal{A}_{l}$ stands for the variation of $\mathcal{A}_{l}$ due to $\delta_{k}$-terms in the variation of $H$, and "t.d.n" are the coefficients of the expansion of the total derivative $(229)$ in the power series in $\hbar$. Explicitly, one gets

$$
[\text { t.d. }]_{0}=0,[\text { t.d. }]_{1}=\epsilon \overleftrightarrow{\nabla} \theta(H),[\text { t.d. }]_{2}=\frac{2}{3} H \overleftrightarrow{\nabla}\left(\delta^{\prime}(H)(a \overleftrightarrow{\nabla} H)\right), \ldots
$$

each [t.d. $]_{k}$ is an expression of the form $A \stackrel{\leftrightarrow}{\nabla} B$, i.e. a total derivative indeed. All the total derivative terms vanish at infinity by the following reasons. General structure of these terms is [t.d.] $=\frac{\partial}{\partial x^{m}} f^{m}+\frac{\partial}{\partial p_{m}} g_{m}$. After integrating by $(x, p)$ these terms are transformed in integrals by $x$-infinity $(f$-term) and by $p$-infinity $(g$-term). As $x \rightarrow \infty$, the $f$-term vanishes as it contains gauge parameters which have compact support in $x$ space and vanish, together with all their derivatives, outside the support domain. As $p \rightarrow \infty$, the total derivative vanishes as $g_{m}$ has the form $\delta^{k}(H) \tilde{g}_{m}, k=0,1,2, \ldots$ localized at the constraint surface $H=0$ which does not contain $p$-infinity, and therefore $g^{m}(p \rightarrow \infty)=0$. This reasoning applies in the framework of the semiclassical expansion. Thus, one has

Proposition 5 If $H \in \mathcal{C}_{p}$ then the "quantized volume" action (215,221) is gauge invariant, at least perturbatively in $\hbar$, w.r.t. gauge transformations (209) with gauge parameters being power series in momenta with coefficients being smooth tensor fields with a compact support in $x$-space.

The conditions of this theorem may be relaxed but we do not consider this task here. For our needs the theorem (5) is sufficient. Indeed, as we saw above there exists a natural choice of $H$ where the constraint surface is compact, namely, $\mathrm{H}=\mathrm{H}_{2}$ where the metric inside $\mathrm{H}_{2}$ is Euclidean and the dilaton is non-negative. General hamiltonians are treated perturbatively like in (224) where it is supposed $\mathcal{C}_{p}$-restrictions are obeyed. Thus, one has

Proposition 6 The "quantized volume" action (215,221) is gauge invariant (perturbatively in $\hbar$ ) for $H$ being the power series of the form (224) with Euclidean metric and $D \geq 0$, provided that tensor fields entering the power series $\Delta H$ are subject to restrictions (which do not reduce the dimension of the space of fields) insuring that the constraint surface $\mathrm{H}_{2}+\Delta H=0$ is compact.

After the integration by momenta is performed in the Euclidean regime (i.e. when $H_{2}$ contains Euclidean metric) the result of integration may be analytically continued, along the lines explained above, to the cases when the metric has another signature. 
In so doing, the gauge invariance is not spoiled as after integration by momenta in Euclidean regime, because check of gauge invariance does not involve the $\mathcal{C}_{p}$-restrictions. Indeed, these restrictions do not impose algebraic constraints on $H^{m(k)}$ and therefore could not insure vanishing of any expression built from $H^{m(k)}$. On the other hand, the variation of the action is zero in the Euclidean regime, therefore, after integration by momenta its vanishing is controlled by the very structure of the Lagrangian expressed in terms of the component fields, this structure of course remains the same whether $H \in \tilde{\mathcal{C}}_{p}$ or not.

\section{Restoration of the low spin data. Low spin ansatz: Weyl-invariant dilaton gravity}

In this section, we show that the low-spin truncation of the "quantized volume" action (221) naturally leads to a Weyl-invariant dilaton gravity.

\subsection{Calculation}

The action has the form

$$
\mathcal{A}[H]=\operatorname{Tr} \theta(H *)=\frac{1}{(2 \pi \hbar)^{d}} \int d^{d} x d^{d} p \theta(H *) .
$$

The formula (221) is rewritten as

$$
\theta(H *)=\theta(H)-\frac{\hbar^{2}}{8}\left[\theta^{\prime \prime}(H) C_{1}+\frac{1}{3} \theta^{\prime \prime \prime}(H) C_{2}\right]+o\left(\hbar^{4}\right)
$$

where

$$
\begin{gathered}
C_{1}=H_{m n} H^{m n}-H_{n}^{m} H_{m}^{n} \\
C_{2}=H_{m} H_{n} H^{m n}+H^{m} H^{n} H_{m n}-2 H^{m} H_{n} H_{n}^{m},
\end{gathered}
$$

where the lower and the upper indices denote the derivatives w.r.t. coordinates $x^{m}$ and momenta $p_{m}$, respectively. In this section we perform the calculation of this action for the particular "low spin" Hamiltonian of the second order

$$
H_{2}=D(x)-g^{m n}(x) p_{m} p_{n},
$$

representing general gravity+Maxwell+dilaton background. To simplify the calculation we set zero the Maxwell field $A_{m}(x)$. As we argued above, in the case $D \geq 0$ and if the metric is Euclidean what we suppose henceforth in this section, the Hamiltonian (234) is a simplest example when $H \in \mathcal{C}_{p}$, therefore all integrals will converge. 
In the case under consideration, the last formula turns to

$$
\begin{gathered}
C_{1}=\left(D_{, m n}-g_{, m n}^{k l} p_{k} p_{l}\right)\left(-2 g^{m n}\right)-4 g_{, m}^{n k} p_{k} g_{, n}^{m l} p_{l} \\
C_{2}=\left(D_{m}-g_{, m}^{k l} p_{k} p_{l}\right)\left(D_{, n}-g_{, n}^{k^{\prime} l^{\prime}} p_{k^{\prime}} p_{l^{\prime}}\right)\left(-2 g^{m n}\right)+ \\
+4\left(D_{, m n}-g_{, m n}^{k l} p_{k} p_{l}\right) g^{m r} g^{n s} p_{r} p_{s}-8 g^{m k} p_{k}\left(D_{n}-g_{, n}^{e f} p_{e} p_{f}\right) g_{m}^{n r} p_{r} .
\end{gathered}
$$

It is seen one has to calculate integrals like

$$
I_{m_{1} \ldots m_{s}}^{(k)}(x)=\int d^{d} p \theta^{(k)}\left(H_{2}\right) p_{m_{1} \ldots} \ldots p_{m_{s}} .
$$

In fact only even-s integrals are to be calculated, because odd-s integrals equal zero. The simplest integral, $I^{(0)}$, which reproduces the classical part of the semiclassical expansion (221) is

$$
I^{(0)}(x)=\int d^{d} p \theta\left(D-p^{2}\right) .
$$

Repeating the reasoning from Sec. (5.4) let us note that the surface $H_{2}=D-p^{2}=0$ splits the phase space into two domains: "inside", $H_{2}>0 \leftrightarrow p^{2}<2 D$ and "outside" $H_{2}<0 \leftrightarrow p^{2}>D$. By definition of the $\theta$-function, the integrand is zero outside. Therefore, all the integrals (230) are well-defined as the actual integration runs over a compact domain.

The integral (237) is equal to the volume of the domain bounded by the constraint surface $H_{2}=0 . I^{(k)}$ are obtained via $I^{(0)}$ by the successive differentiation by dilaton $D$,

$$
I_{m_{1} \ldots m_{s}}^{(k)}(x)=\left(\frac{\partial}{\partial D}\right)^{k} I_{m_{1} \ldots m_{s}}^{(0)}(x)
$$

while $I_{m_{1} \ldots m_{s}}^{(0)}(x)$ are encoded by the generating function

$$
G_{d}(l)=\sum_{s=0}^{\infty} \frac{i^{s}}{s !} I_{m_{1} \ldots m_{s}}^{(0)}(x) l^{m_{1}} \ldots l^{m_{s}}=\int d^{d} p \theta\left(H_{2}\right) e^{i p_{m} l^{m}} .
$$

$G_{d}$ is easily calculated (see App. (DD)) to be

$$
G_{d}(l)=2^{\frac{d}{2}} \pi^{\frac{d}{2}} l^{-\frac{d}{2}} \sqrt{g} D^{\frac{d}{4}} J_{\frac{d}{2}}(l \sqrt{D}),
$$

where $l=\sqrt{l^{2}}, g=\operatorname{det}\left(g_{m n}\right) . J_{\frac{d}{2}}$ is the Bessel's function of the first kind (App. (国)),

$$
J_{k}(z)=\left(\frac{z}{2}\right)^{p} \sum_{k=0}^{\infty} \frac{(-)^{k}}{k ! \Gamma(p+1+k)}\left(\frac{z}{2}\right)^{2 k}
$$

Note that the generating function (240) is a power series in even degrees of $l$, i.e., a power series in $l^{2}$. Expanding both sides of (240) in $l^{m}$ one gets the expressions of integrals (236) in terms of tensor powers of the metric $g_{m n}$. 
Employing (240) and its derivatives by dilaton $D$ (see (564)), one can easily get the answer for any integral (236). In this subsection we need only integrals

$$
\begin{gathered}
\int d^{d} p \theta\left(D-p^{2}\right)=\sqrt{g} v_{d} D^{\frac{d}{2}} \\
\int d^{d} p \theta\left(D-p^{2}\right) p_{m} p_{n}=\sqrt{g} v_{d} D^{\frac{d}{2}+1} \frac{1}{d+2} g_{m n} \\
\int d^{d} p \theta\left(D-p^{2}\right) p_{k} p_{l} p_{k^{\prime}} p_{l^{\prime}}=\sqrt{g} v_{d} D^{\frac{d}{2}+2} \frac{1}{(d+4)(d+2)} G_{k l k^{\prime} l^{\prime}}
\end{gathered}
$$

where

$$
G_{k l k^{\prime} l^{\prime}}=g_{k l} g_{k^{\prime} l^{\prime}}+g_{k k^{\prime}} g_{l^{\prime} l}+g_{k l^{\prime}} g_{l k^{\prime}}
$$

where

$$
v_{d}=\frac{\pi^{\frac{d}{2}}}{\Gamma\left(\frac{d}{2}+1\right)}
$$

is the volume of a unit ball in $d$ dimensions, and the consequences of these integrals obtained by a few differentiations w.r.t. $D$. This is enough for easily writing down the result of integration by momenta for the expression (231):

$$
\begin{gathered}
\mathcal{A}\left[H_{2}\right] \equiv \mathcal{A}\left[D(x), g_{m n}(x)\right]= \\
\frac{v_{d}}{(2 \pi \hbar)^{d}} \int d^{d} x \sqrt{g}\left\{D^{\frac{d}{2}}-\frac{\hbar^{2}}{12} \frac{d}{2}\left(\frac{d}{2}-1\right)\left(\frac{d}{2}-2\right) D^{\frac{d}{2}-3} g^{m n} D_{, m} D_{, n}-\frac{\hbar^{2} d}{8} D^{\frac{d}{2}-1} Y+\right. \\
\left.+\frac{\hbar^{2}}{12} \frac{d}{\sqrt{g}} \partial_{n}\left(\sqrt{g} g^{m n} \partial_{n} D^{\frac{d}{2}-1}\right)\right\}+o\left(\hbar^{4}\right),
\end{gathered}
$$

where $Y$ is an expression composed purely from the metric and its first and second derivatives,

$$
Y=\frac{1}{2} g_{, m n}^{k l} g^{m n} g_{k l}-g_{, m}^{n k} g_{, n}^{m l} g_{k l}+\frac{1}{3} G_{k l k^{\prime} l^{\prime}}\left(-\frac{1}{4} g_{, m}^{k l} g_{, n}^{k^{\prime} l^{\prime}} g^{m n}-\frac{1}{2} g_{, m n}^{k l} g^{m k^{\prime}} g^{n l^{\prime}}\right)
$$

Now we have to discuss the gauge invariance of this action. By construction, general action is gauge-invariant w.r.t. gauge transformations (209). In the case of the low spin ansatz (234) one has to restrict to the subalgebra of gauge transformations which preserves the second-order ansatz. This subalgebra, $\mathcal{G}_{0}$ is spanned by $\epsilon=\varepsilon(x)+\xi^{m}(x) p_{m}$ and $a=\alpha(x)$, parameterizing infinitesimal $U(1)$ (phase) transformations, $x$-diffeomorphisms and Weyl dilations, respectively. The commutation relations are quite the same as in the classical case due to special properties of the Weyl symbol. Nevertheless, the action of $\mathcal{G}_{0}$ on the components of the Hamiltonian $H_{2}$ acquires quantum corrections. One has

$$
\delta_{\varepsilon, a} H_{2}=\left[\alpha, H_{2}\right]_{+}+\frac{1}{i \hbar}\left[\varepsilon, H_{2}\right]
$$


For the case at hand the $\hbar$ series terminate at $\hbar^{2}$ terms. One has

$$
\begin{gathered}
\delta_{\varepsilon} D=-\xi^{m} \partial_{m} D-\frac{\hbar^{2}}{4}\left(\partial_{m} g^{a b}\right) \partial_{a} \partial_{b} \xi^{m}+2 \alpha D+\frac{\hbar^{2}}{2} g^{m n} \alpha_{, m n} \\
\delta_{\varepsilon} A_{m}=-\xi^{k} \partial_{k} A_{m}+A_{k} \partial_{m} \xi^{k}-\partial_{m} \varphi \\
\delta_{\varepsilon} g^{m n}=-\xi^{k} \partial_{k} g^{m n}+g^{m k} \partial_{k} \xi^{n}+g^{n k} \partial_{k} \xi^{m}+2 \alpha g^{m n},
\end{gathered}
$$

where $A_{m}=g_{m n} A^{m}$. Here $\hbar^{2}$-independent terms represent standard $U(1)$ transformations, $x$-diffeomorphisms and Weyl dilations, while $\hbar^{2}$-correction to the dilaton's gauge transformations prohibits its interpretation of a scalar field. Note that these gauge transformations are already exact expressions for the gauge transformations. They form the closed algebra, being isomorphic to its classical limit. It appears there exists the redefinition of variables such that the new variables transform in a standard way, i.e. as follows

$$
\begin{gathered}
\delta_{\varepsilon} D^{\prime}=-\xi^{m} \partial_{m} D^{\prime}+2 \alpha D^{\prime} \\
\delta_{\varepsilon} A_{m}^{\prime}=-\xi^{k} \partial_{k} A_{m}^{\prime}+A_{k}^{\prime} \partial_{m} \xi^{k}-\partial_{m} \varphi \\
\delta_{\varepsilon} g^{\prime m n}=-\xi^{k} \partial_{k} g^{\prime m n}+g^{\prime m k} \partial_{k} \xi^{n}+g^{\prime n k} \partial_{k} \xi^{m}+2 \alpha g^{m n}
\end{gathered}
$$

The redefinition reads (see Appendix (F))

$$
\begin{gathered}
D^{\prime}=D-\frac{\hbar^{2}}{4}\left(Y-\frac{d-4}{3(d-1)} R\right), \\
g^{\prime m n}=g^{m n}, A^{\prime m}=A^{m},
\end{gathered}
$$

where $Y$ is from (246) while $R$ is a scalar curvature constructed of the Riemann tensor $R_{n k l}^{m}$ of the metric $g_{m n}$ by the rule $R=g^{m n} R_{m k n}^{k}$.

The action (245) is to be expressed in terms of new "standard" variables. Then it should be a action of Weyl-invariant dilaton gravity, described in Sec. (2.1), because the gauge laws for $D^{\prime}, g^{\prime m n}$ just coincide with those of (29). The result is

$$
\begin{gathered}
\mathcal{A}\left[H_{2}\right] \equiv \mathcal{A}\left[D^{\prime}(x), g_{m n}(x)\right]=\frac{v_{d}}{(2 \pi \hbar)^{d}} \int d^{d} x \sqrt{g}\left\{D^{\prime \frac{d}{2}}+\right. \\
-\frac{\hbar^{2} d(d-4)}{24}\left(\frac{d-2}{4} D^{\prime \frac{d}{2}-3} g^{m n} D_{, m}^{\prime} D_{, n}^{\prime}+\frac{1}{d-1} D^{\prime \frac{d}{2}-1} R\right)+ \\
\left.+\frac{\hbar^{2}}{12} \frac{d}{\sqrt{g}} \partial_{n}\left(\sqrt{g} g^{m n} \partial_{n} D^{\prime \frac{d}{2}-1}\right)\right\}+o\left(\hbar^{4}\right),
\end{gathered}
$$

In the last formula, the first row is the classical, $\hbar^{0}$, term, which does not contain spacetime derivatives of the dynamical fields. It has clear meaning of the cosmological term. 
The dependence of the cosmological term on the dilaton exhibits the fact that, by the very construction, it represents the volume of the $d$-ball of radius $\sqrt{D}$ bounded by the surface $H_{2}=0$ in the momentum space.

According to our predictions, the derived action is nothing but the action of Weyl invariant dilaton gravity (30), described in the beginning of the paper in Sec. (2.1). Evidently, the inclusion of a nonzero Maxwell field $A_{m}(x)$ easily comes along the same lines. The higher orders in $\hbar$ may be computed along the same lines. As the gauge transformations of the modified dilaton $D^{\prime}$ and the metric $g^{m n}$ are just the standard diffeomorphisms while the action is gauge invariant by construction, each $\hbar^{2 k}$ term of the semiclassical expansion will be given (modulo total derivatives) by Weyl-invariant combinations of the scalar $D^{\prime}$, Riemann curvature and their covariant derivatives, with the total number of derivatives of $D^{\prime}$ and $g^{m n}$ being equal $2 k$. Thus we have recovered in our approach the low spin data of Sec. (2.1) and have shown that

Proposition 7 The low-spin truncation of the "quantized volume" action (221) provides, in the Euclidean regime, Weyl-invariant dilaton gravity with cosmological term given by dilaton potential.

\subsection{Comparison to Schwinger-Dewitt technique}

There exists another interpretation of the redefinition (250), that leads us naturally to conjecture about the form of the invariant induced action in even dimensions.

Namely, let us recall that the standard tensor fields, dilaton and metric, of Weylinvariant gravity are the components of the second-order operator

$$
\mathbf{H}=g^{m n} \hat{\nabla}_{m} \hat{\nabla}_{m}-\frac{d-2}{4(d-1)} R+D^{\prime}(x)
$$

governing the quadratic part of the action of free conformal scalar

$$
S_{\mathbf{H}}[\varphi]=\int d^{d} x \sqrt{g} \chi^{*} \mathbf{H} \chi .
$$

where $g=\operatorname{det} g_{m n}$. $\mathbf{H}$ scales as

$$
\mathbf{H} \rightarrow e^{\left(1+\frac{d}{2}\right) a(x)} \mathbf{H} e^{\left(1-\frac{d}{2}\right) a(x)}
$$

w.r.t. Weyl dilations

$$
g^{m n} \rightarrow e^{2 a(x)} g^{m n}, D^{\prime} \rightarrow e^{2 a(x)} D^{\prime}
$$

so that the action (253) is Weyl invariant provided $\chi \rightarrow e^{\left(-1+\frac{d}{2}\right) a(x)} \chi$. 
Clearly, the wave operator $\mathbf{H}$, scalar $\chi$ and the action $S_{\mathbf{H}}[\chi]$ are related to some Hamiltonian $\hat{H}$, wave function $\psi(x)$ and the coupling (133) by the map

$$
\begin{gathered}
S_{\mathbf{H}}[\chi]=S_{H}[\psi] \\
\mathbf{H}=g^{-\frac{1}{4}}(x) \hat{H} g^{\frac{1}{4}}(x), \quad \chi(x)=g^{-\frac{1}{4}}(x) \psi(x),
\end{gathered}
$$

Given $\mathbf{H}$, this map gives a second-order operator $\hat{H}$ which starts from $g^{m n}(x) \partial_{m} \partial_{n}$. It proves that by the $x$-diffeomorphism covariance and Weyl covariance, this operator is nothing but the operator with the Weyl symbol (let $\hbar=1$ )

$$
H=-g^{m n}(x) p_{m} p_{n}+D(x)=-g^{m n}(x) p_{m} p_{n}+D^{\prime}+\frac{1}{4}\left(Y-\frac{d-4}{3(d-1)} R\right),
$$

where the relation of $D(x)$ and $D^{\prime}(x)$ is from Eq. (250).

Note that the origin of the map (256) is clear by the following reasons. In the Weyl symbol, $x$-diffeomorphisms are generated by the generating function

$$
\omega=\frac{i}{\hbar} \xi^{m}(x) p_{m}
$$

which is the Weyl symbol of the operator

$$
\hat{\omega}=\frac{1}{2}\left(\xi^{m} \partial_{m}+\partial_{m} \xi^{m}\right)=\xi^{m}(x) \partial_{m}+\frac{1}{2}\left(\partial_{m} \xi^{m}(x)\right) .
$$

The latter operator defines representation of general coordinate transformations characteristic for the scalar density of weight $\frac{1}{2}$, that is manifested by the $\frac{1}{2} \partial_{m} \xi^{m}$ addition to ordinary vector field $\xi^{m} \partial_{m}$. The $g^{\frac{1}{4}}$ factors in (256) just compensate this addition rendering infinitesimal general coordinate transformations to be ordinary vector fields. To summarize, $x$-diffeomorphisms, which look as $\delta H=-\frac{i}{\hbar}\left[\xi^{m} p_{m}, H\right]_{*}$ in the symbol approach and lead to unusual transformation laws (248) for the dilaton $D$, in terms of $\mathbf{H}$ are just ordinary vector fields which transform $D^{\prime}(x)$ as a scalar. In so doing, $D^{\prime}$ is just a scalar part of the generally covariant differential operator $\mathbf{H}$.

Having established the map between the symbol $H(x, p)$ and operator $\mathbf{H}$ one can use the Schwinger-Dewitt technique for evaluating the expression

$$
Q(i \tau)=\operatorname{Tr} \exp (i \tau \mathbf{H})
$$

and compare the result with our calculations in symbol formalism. The benefit of using the Schwinger-Dewitt approach is that $\mathbf{H}$ is parameterized by ordinary tensor fields and $Q(i \tau)$ is expressed as an integral of the covariant expression 31 built from Riemann curvature, dilaton and their covariant derivatives. Namely, let

$$
\mathbf{H}=g^{m n} \hat{\nabla}_{m} \hat{\nabla}_{m}-\frac{1}{6} R+P(x)+m^{2} \Rightarrow,
$$


where

$$
P(x)+m^{2}=D^{\prime}(x)+\frac{d-4}{12(1-d)} R
$$

Then the Schwinger-Dewitt technique provides the expression [31]

$$
\operatorname{Tr} \exp (i \tau \mathbf{H})=\frac{e^{i m^{2} \tau}}{(4 \pi i \tau)^{\frac{d}{2}}} \sum_{n=0}^{\infty}(i \tau)^{n} \int d^{d} x \sqrt{g} a_{n}(x, x)
$$

where $a_{n}(x, x)$ are built from $P(x)$, Riemann curvature and their covariant derivatives, 31]

$$
\begin{gathered}
a_{0}(x, x)=1, \alpha_{1}(x, x)=P, \\
a_{2}(x, x)=\frac{1}{180}\left(R_{m n k l} R^{m n k l}-R_{m n} R^{m n}+\square R\right)+\frac{1}{2} P^{2}+\frac{1}{6} \square P,
\end{gathered}
$$

and so on.

Our action is given by the formula

$$
\mathcal{A}[H]=\operatorname{Tr} \theta(\hat{H})=\operatorname{Tr} \frac{1}{2 \pi i} \int_{-\infty}^{\infty} \frac{d \omega}{\omega-i \varepsilon} e^{i \omega \hat{H}}
$$

Suppose changing the order of integration by $\omega$ with " $T r$ " operations is a legal operation 10 one gets

$$
\frac{1}{2 \pi i} \int_{-\infty}^{\infty} \frac{d \omega}{\omega-i \varepsilon} Q(i \omega)=\frac{1}{2 \pi i} \int_{-\infty}^{\infty} \frac{d \omega}{\omega-i \varepsilon} \frac{e^{i m^{2} \omega}}{(4 \pi i \omega)^{\frac{d}{2}}} \sum_{n=0}^{\infty}(i \omega)^{n} \int d^{d} x \sqrt{g} a_{n}(x, x)
$$

Let $d$ be even. For $m^{2}>0$ the integrals by $\omega$ are calculated easily as integration contour may be continued to the closed contour in a full complex plane by adding the point $\operatorname{Im} \omega=+\infty$,

$$
\mathcal{A}[H]=\frac{1}{(4 \pi)^{\frac{d}{2}}} \sum_{n=0}^{\frac{d}{2}} \frac{\left(m^{2}\right)^{\frac{d}{2}-n}}{\left(\frac{d}{2}-n\right) !} \int d^{d} x \sqrt{g} a_{n}(x, x) .
$$

Using the identification (262) one can see that this expression coincides, modulo boundary terms, with that obtained in the Weyl symbol formalism (251). But the expression (267) is nothing but the time-independent term of the decomposition of the trace of the evolution operator,

$$
\mathcal{A}[H]=\operatorname{Tr} \theta(\hat{H})=\lim _{\tau \rightarrow 0^{+}} \operatorname{Tr} \exp (i \tau \mathbf{H})
$$

We expect analogous phenomenon takes place for general Hamiltonians which start from small positive dilaton.

Let us comment on general coordinate invariance in our model. Of course, the action is general coordinate invariant as $x$-diffeomorphisms form a subalgebra of the gauge algebra of the theory. On the other hand, the coefficients of the decomposition of the

\footnotetext{
${ }^{10}$ In general such a changing is hard to justify. We nevertheless omit the detailed discussion of this issue supposing the changing of contours is meaningful at least for a wide class of hamiltonians.
} 
Weyl symbol $H(x, p)$ in power series in momenta are not ordinary tensors but acquire quantum corrections to ordinary tensor transformation laws (154). Luckily, there exists the parameterization of general differential operator in terms of ordinary tensor fields. It is achieved by transferring to the new operator $\mathbf{H}(x, \nabla)$ being a power series in covariant derivatives. Namely, given general Hamiltonian with Weyl symbol

$$
H(x, p)=\sum_{s=0}^{\infty} H^{m(s)}(x) p_{m_{1}} \ldots p_{m_{s}}
$$

one has differential operators

$$
\hat{H}=\sum_{s=0}^{\infty}(-i \hbar)^{s} H^{m(s)}(x) \partial_{m_{1}} \ldots \partial_{m_{s}}+\ldots
$$

(see (598,600) for exact formula), and

$$
\mathbf{g}^{-\frac{1}{4}}(x) \hat{H} \mathbf{g}^{\frac{1}{4}}(x)=\mathbf{H}=\sum_{s=0}^{\infty}(-i \hbar)^{s} \mathbf{H}^{m(s)}(x) \nabla_{m_{1}} \ldots \nabla_{m_{s}}
$$

where the inverse metric is nothing but $\mathbf{H}^{m(2)}(x)$ and $\mathbf{g}=\sqrt{\operatorname{det} \mathbf{g}_{\mathbf{m n}}}$. Thus one gets the map from the set of tensor fields $\mathbf{H}^{m(s)}$ comprising power series $\mathbf{H}(\mathbf{x}, \nabla)$ to the set of coefficients $H^{m(s)}$ of decomposition of $H(x, p)$ in momenta, the latter transforms w.r.t. $x$-diffeomorphisms according to (154). Thus any $x$-diffeomorphism invariant expression built from $H^{m(s)}$ may be rewritten as invariant one in terms of $\mathbf{H}^{m(s)}$ i.e. as an integral

$$
\int d^{d} x \sqrt{\mathbf{g}} F\left(\nabla_{r_{1}} \ldots \nabla_{r_{k}} R_{e f g h}, \nabla_{q_{1}} \ldots \nabla_{q_{l}} \mathbf{H}^{m(s)}, \ldots\right)
$$

of a function of Riemann curvature, $\mathbf{H}^{m(s)}$ and their covariant derivatives. Rewriting of the action in terms of tensor fields $\mathbf{H}^{m(s)}$ and their covariant derivatives is what is likely more simply performed in the Schwinger-Dewitt approach.

Despite the Schwinger-Dewitt technique is less elaborated in the case of general operators (271) one can make perturbative calculations along the lines of $w$-decomposition of Sec. (5.4), i.e. taking the higher spin part as a small fluctuation. In so doing, one will always get $Q(i \tau)$ as a power series of the form

$$
Q(i \tau)=(4 \pi i \tau)^{-\frac{d}{2}} e^{i m^{2} \tau} \sum_{n=0}^{\infty} \int d^{d} x \sqrt{g} a_{n}
$$

where $a_{n}$ are covariant expressions built from Riemann curvature, tensor fields $\mathbf{H}^{m(s)}$ and their covariant derivatives which reduce to ordinary Schwinger-Dewitt coefficients (264) when higher rank fields are switched off. Then as $m^{2}>0$ the integration by $\omega$ in (266) may be always continued to run over the closed contour around origin in the complex plane and thereby gives the simple residue of the expression $Q(z)$ at the origin or just 
$Q(0)$. Thus, at least in the framework of the $w$-expansion one has the formula (268) for an arbitrary Hamiltonian (in even dimensions). This provides an alternative definition of the action of the conformal higher spin theory.

But the same expression provides the logarithmically divergent term of the one-loop effective action,

$$
\begin{aligned}
& -\frac{1}{2} \operatorname{Tr} \operatorname{Ln} \hat{H}=\frac{1}{2} \int_{\lambda=\mu}^{\infty} \frac{d \lambda}{\lambda} \operatorname{Tr} e^{\lambda \hat{H}}=-\frac{1}{2}(\ln \mu) Q(0)+ \\
& + \text { power divergencies in } \mu+\text { regular terms in } \mu .
\end{aligned}
$$

In this sense, in even dimensions, the action of the model of the paper is nothing but the logarithmically divergent term in the one-loop effective action of complex scalar field for an arbitrary background wave operator $\hat{H}$. This means the invariant induced action is a logarithmically divergent part of true induced action $-\frac{1}{2} \operatorname{Tr} L n \hat{H}$ of the quantized particle.

\subsection{Conformally flat vacuum}

According to the derivation of Sec. (2.1), in $d>2$, the theory (231,251) possesses the vacuum of the form

$$
D^{\prime}=0, A_{m}=0, g^{m n}=\eta^{m n}
$$

with $\eta^{m n}$ being flat metric, or, equivalently, of the form

$$
H=-p^{2}
$$

As the theory is Weyl-invariant, the Weyl-dilated configuration

$$
\begin{gathered}
H_{a(x)}=e^{a(x)} *\left(-p^{2}\right) * e^{a(x)}=-e^{2 a(x)}\left(p^{2}-\frac{\hbar^{2}}{4} \square a(x)\right) \Leftrightarrow \\
D^{\prime}=0, A_{m}=0, g^{m n}=e^{2 a(x)} \eta^{m n}
\end{gathered}
$$

is a vacuum either. Therefore, the Hamiltonian $H_{a(x)}$ represents a family of conformally flat vacua of the low spin truncated theory. Note that among the vacua (276) there are anti-de Sitter and de Sitter spaces corresponding to the following choices of the conformal factor

$$
e^{2 a_{A d S}}=\left(1-R^{-2} x^{2}\right)^{2}, e^{2 a_{d S}}=\left(1+R^{-2} x^{2}\right)^{2},
$$

where $R$ is "radius" of AdS or dS space. It is natural to wonder if the vacuum (276) can be a vacuum of the whole non-truncated theory. The answer is yes, the proof is the subject of the next section. 


\section{Conformally flat vacuum.}

Consider the perturbative expansion of the "quantized volume" action (221) around the configuration

$$
\bar{H}=D-p^{2},
$$

where $D \geq 0$ and $p^{2}$ is built with flat Euclidean metric. To this end one represents general $H$ as

$$
H(x, p)=\bar{H}+e h(x, p)
$$

where $h(x, p)$ is a fluctuation, and expands the action (221) in powers of $e h$,

$$
\mathcal{A}[\bar{H}+h]=\mathcal{A}[\bar{H}]+e \mathcal{A}_{1}[h]+e^{2} \mathcal{A}_{2}[h]+\ldots
$$

The zero order term is easily calculated as any *-function of $\bar{H}$ amounts to ordinary function,

$$
F(\bar{H} *)=F(\bar{H})
$$

Therefore, the action is given by the classical term while the quantum corrections vanish. The result is

$$
\mathcal{A}[\bar{H}]=\frac{1}{(2 \pi \hbar)^{d}} \int d^{d} x d^{d} p \theta\left(D-p^{2}\right)=\frac{v_{d}}{(2 \pi \hbar)^{d}} D^{\frac{d}{2}} \int d^{d} x
$$

Of course this expression diverges but this is not dangerous for our purposes.

Of our main interest in this section is the $e h$-linear term. This term equals the variation of the action (221), under the variation of the Hamiltonian of the form $\delta H=e h$, around the configuration $H=\bar{H}$. Taking into account that the order of product factors under the sign of trace operation is inessential (up to total derivative terms) one gets

$$
\begin{gathered}
\mathcal{A}[\bar{H}+h]-\mathcal{A}[\bar{H}]=\frac{1}{(2 \pi \hbar)^{d}} \int d^{d} x d^{d} p\{\theta((\bar{H}+e h) *)-\theta(\bar{H} *)\}= \\
\frac{1}{(2 \pi \hbar)^{d}} \int d^{d} x d^{d} p \delta(\bar{H} *) * e h+\text { tot. derivative }=\frac{1}{(2 \pi \hbar)^{d}} \int d^{d} x d^{d} p \delta(\bar{H}) e h+\text { tot. derivative, }
\end{gathered}
$$

where in the last equality two *-products turn to ordinary products because of the Eq. (198) and "simple multiplication" rule (200). The total derivatives by $x$ are transformed into the integrals by $x$-infinity and vanish as $h$ is required to vanish at $x$-infinity. The total derivatives by $p$ may be shown to vanish either, as they are proportional to derivatives of delta-function $\delta(\bar{H})$ (that may be shown along the same lines as in Sec. (5.5)).

The main part of the variation, the expression

$$
\frac{1}{(2 \pi \hbar)^{d}} \int d^{d} x d^{d} p \delta(\bar{H}) h(x, p)
$$


presents the integral by the constraint surface $\bar{H}=0$ being $d-1$-sphere. This expression is easy calculable to be

$$
\frac{1}{(2 \pi \hbar)^{d}} \int d^{d} x d^{d} p \delta(\bar{H}) h(x, p)=\frac{1}{(2 \pi \hbar)^{d}} \sum_{s=0}^{\infty} D^{\frac{d}{2}-1+s} d_{s} \int d^{d} x h^{m_{1} \ldots m_{s}}{ }_{m_{1} \ldots m_{s}}(x),
$$

where $d_{s}$ are non-zero coefficients which may be extracted e.g. from generating functions (564).

Now consider the case

$$
D=0
$$

Then the variation vanishes provided $d>2$ and thus the configuration $H=-p^{2}$ is a solution of the classical equations of motion of the "quantized volume" action, i.e. it is a vacuum. This vanishing of the variation has a transparent meaning as $\sqrt{D}$ is the radius of the sphere $\bar{H}=0$ and therefore the limit $D \rightarrow 0$ corresponds to the case when the sphere shrinks to a point. As a consequence, the "quantized volume" as well as its variation, vanishes in even dimensions (in $d>2$ ). At the same time, in odd dimensions the $w$-decomposition of the invariant induced action in the sense of Sec. (5.4) contains all negative powers of the dilaton, therefore, the fact that the linear variation cancels is a less trivial phenomena which manifests that all terms in the linear variation of $\mathcal{A}[H]$ with negative powers of $D$ become total derivatives as $D \rightarrow 0$. Thus, we get the

Proposition 8 In $d>2$, the Hamiltonian $H=-p^{2}$ is a solution of the classical equations of motion for the "quantized volume" action (221).

Now one recalls Proposition (2) to derive

Proposition 9 In $d>2$, the quadratic in $h$ terms of the perturbative expansion of the "quantum volume" are described by a sum of deformed conformal higher spin theories with $\mu^{2}=-\frac{\hbar^{2}}{4} \square$.

In the next section, by direct calculation we show this is the case indeed. Thereby, the "quantized volume" is a consistent action for the (Euclidean phase of) conformal higher spin theory.

As the whole theory is invariant w.r.t. Weyl dilations, one gets the family of vacua

$$
H_{a(x, p)}=-e^{a(x, p) *} * p^{2} * e^{a(x, p) *},
$$

We call any vacuum of the form a conformally flat vacuum. Below we perform all the calculations in the case $a=0$, but one has to keep in mind that the results of calculations are related by the gauge transformation to another conformally flat vacua, e.g. $d$-dimensional de Sitter or anti-de Sitter spaces (277). 


\section{Restoration of the linearized data. Perturbative expansion around the conformally flat vacuum}

In this section, we consider perturbative expansion and linearization of the theories (221), firstly around an arbitrary vacuum solution, and then around the conformally flat vacuum. We uncover, at the quadratic order, a sum of conformal higher spin theories with $s=$ $0,1,2,3, \ldots$ that presents our main result.

\subsection{General perturbative expansion.}

Consider the functional

$$
A_{F}[H *]=\operatorname{Tr} F(H *)
$$

and perform its expansion in perturbative series around a particular point $H=A$. This means representing general Hamiltonian as

$$
H=\bar{H}+e h,
$$

and expand the action (288) in Tailor series in $h$. This is achieved as follows. First, we recall the representation of the function $F(H *)$ in Fourier-Laplace type series (195), which gives

$$
\operatorname{Tr} F(H *)=\int_{C} d \lambda \tilde{F}(\lambda) \operatorname{Tr} \exp (-\lambda H *),
$$

where $\lambda \in C$ while $C$ is a contour in a complex plane.

One expands the expression $\operatorname{Tr} u(-\lambda)=\operatorname{Tr} \exp (-\lambda H *)$ and then integrates by $\lambda$. The expansion of the exponent is performed by making use of the formula

$$
\exp (-\lambda(\bar{H}+e h) *)=\exp (-\lambda \bar{H} *) * T \exp \left(-\int_{0}^{\lambda} e^{\lambda \bar{H} *} * e h * e^{-\lambda \bar{H} *}\right)
$$

which is proved easily by the differentiation by $\lambda$. $T$ denotes the $T$-ordering,

$$
T\left(B_{1}\left(\lambda_{1}\right) B_{2}\left(\lambda_{2}\right)\right)=\theta\left(\lambda_{1}-\lambda_{2}\right) B_{1}\left(\lambda_{1}\right) B_{2}\left(\lambda_{2}\right)+\theta\left(\lambda_{2}-\lambda_{1}\right) B_{2}\left(\lambda_{2}\right) B_{1}\left(\lambda_{1}\right) .
$$


According to this formula, the functional (288) is equal, up to $h^{3}$ terms,

$$
\begin{gathered}
\mathcal{A}_{F}[H *]=\operatorname{Tr} F((\bar{H}+e h) *)= \\
\int_{C} d \lambda f(\lambda) \operatorname{Tr} e^{-\lambda(\bar{H}+e h) *}=\int_{C} d \lambda f(\lambda) e^{-\lambda \bar{H} *} * \operatorname{Texp}\left(-\int_{0}^{\lambda} e^{\lambda \bar{H} *} * e h * e^{-\lambda \bar{H} *}\right)= \\
=\mathcal{A}_{0}[\bar{H}]+e \mathcal{A}_{1}[\bar{H}, h]+e^{2} \mathcal{A}_{2}[\bar{H}, h]+e^{3} \mathcal{A}_{3}[\bar{H}, h]+o\left(h^{4}\right) \\
\mathcal{A}_{0}[\bar{H}]=\operatorname{Tr} F(H *) \\
\mathcal{A}_{1}[\bar{H}, h]=\operatorname{Tr} F^{\prime}(H *) * h \\
\mathcal{A}_{2}[\bar{H}, h]=\int_{C} d \lambda f(\lambda) \operatorname{Tr}^{-\lambda \bar{H} *} * \int_{0}^{\lambda} d \tau_{1} \int_{0}^{\tau_{1}} d \tau_{2} e^{\tau_{1} \bar{H} *} * h * e^{-\tau_{1} \bar{H} *} * e^{\tau_{2}} \bar{H} * * h * e^{-\tau_{2} \bar{H} *} \\
\mathcal{A}_{3}[\bar{H}, h]=-\frac{1}{2} \int_{C} d \lambda f(\lambda) \operatorname{Tr} e^{-\lambda \bar{H} *} * \\
* \int_{0}^{\lambda} d \tau_{1} \int_{0}^{\tau_{1}} d \tau_{2} \int_{0}^{\tau_{2}} d \tau_{3} e^{\tau_{1} \bar{H} *} * h * e^{-\tau_{1} \bar{H} *} * e^{\tau_{2} \bar{H} *} * h * e^{-\tau_{2} \bar{H} *} * e^{\tau_{3} \bar{H} *} * h * e^{-\tau_{3} \bar{H} *}
\end{gathered}
$$

In what follows, we apply these formulae to the case

$$
F(\sigma)=\theta(\sigma)
$$

what is equivalent to choosing the function $\tilde{F}(\lambda)$ and the integration contour as follows,

$$
\tilde{F}(\lambda)=\frac{1}{2 \pi i \lambda}, \lambda=-\varepsilon+i \tau, \tau \in \mathbf{R}, \varepsilon \rightarrow 0^{+}
$$

We also make use of the notation

$$
a_{d}=\frac{1}{(2 \pi \hbar)^{d}}, b_{d}=(\pi \hbar)^{d}
$$

It is worth discussing briefly where the leading terms of the conformal higher spin models will come from. The calculation we perform is in the framework of expansion in two parameters $\hbar$ and $e$, the former counts the number of $x$-derivatives and the latter counts "the number of tails" i.e. the degree of $h$, such that a term $\sim \hbar^{2 k} e^{l}$ is a homogeneous polynomial in $h$ of degree $l$ with the total number of $x$-derivatives equal to $2 k$. Using integration technique explained in Sec. (6.1) and App. (D) it may be readily shown that after taking all integrals by momenta, the invariant induced action has a form like

$$
\mathcal{A}\left[D-p^{2}+e h\right] \sim \int d^{d} x \sum_{r} \sum_{l=0}^{\infty} e^{l} D^{-r}(\hbar \partial)^{d-2 l+2 r+\sum_{i=0}^{l} s_{i}} h_{s_{1}} \ldots h_{s_{l}},
$$


where partial derivatives act in a diverse way on the rank- $s_{1} \ldots s_{l}$ components of the fluctuation. In even dimensions terms with negative powers of $D$ are zero by the reasons discussed in Sec. (5.4) while in odd dimensions arbitrary negative powers of $D$ are available. The terms with negative degrees of $x$-derivatives are absent by the very construction, as we are doing semiclassical decomposition in $\hbar \partial$, this implies summations are actually restricted to the domain $2 r \geq-d+2 l-\sum_{i=0}^{l} s_{i}$. Of our main interest $D=0$ case,

$$
\lim _{D \rightarrow 0^{+}} \mathcal{A}\left[D-p^{2}+e h\right] \sim \int d^{d} x \sum_{l=0}^{\infty} e^{l}(\hbar \partial)^{d-2 l+\sum_{i=0}^{l} s_{i}} h_{s_{1}} \ldots h_{s_{l}},
$$

While in even dimensions the latter expression is a straightforward limit of $D>0$ one, in odd dimensions the situation is more subtle, the limit $D \rightarrow 0$ exists in the sense this may be a vacuum but not in the framework of perturbative expansion, just like the point $\sigma=0$ is not a good point for expansion of the function $f(\sigma)=\sigma^{\frac{k}{2}}, \kappa=1,2,3, \ldots$. Our calculations will naturally incorporate odd-dimensional case, despite it is only the even-dimensional case when the perturbative expansion is well-defined in a usual sense.

Consider the quadratic action, i.e. $l=2$, and let $s_{1}=s_{2}=s$. Then one has

$$
\lim _{D \rightarrow 0^{+}} \mathcal{A}\left[D-p^{2}+e h\right] \sim e^{2} \int d^{d} x \sum_{s=0}^{\infty}(\hbar \partial)^{d-4+2 s} h_{s} h_{s}+\ldots+o\left(e^{3} h^{3}\right),
$$

where "..." stands for non-diagonal terms. Terms $\sim(\hbar \partial)^{d-4+2 s} h_{s} h_{s}$ are nothing but the leading terms of free conformal higher spin models of spin $s$. It is seen that the conformal spin- $s$ model originates from the $d-4+2 s$-th quantum correction to the classical volume of the domain bounded by the constraint surface, in the limit $D \rightarrow 0$ when the surface shrinks to a point in the momentum space.

\subsection{Calculation of the quadratic part of the action}

Calculate the quadratic term in the expansion (293) around the vacuum solution

$$
\bar{H}=-p^{2} .
$$

One has

$$
\begin{gathered}
e^{\lambda p^{2} *}=e^{\lambda p^{2}} \\
e^{\lambda p^{2} *} * h(x, p) * e^{-\lambda p^{2} *}=h(x-2 i \hbar \lambda p, p) .
\end{gathered}
$$

After making use of the trace property (198), the "simple multiplication" property (200) and making the change of variables

$$
t=\tau_{1}-\tau_{2}, \tau_{1}=\tau_{1}
$$


one rewrites the quadratic action form (293) as

$$
\mathcal{A}_{2}=-a_{d} \int_{C} d \lambda \tilde{F}(\lambda) \int d x d p e^{\lambda p^{2}} \int_{0}^{\lambda} d \tau_{1} \int_{0}^{\tau_{1}} d t h(x, p) * h(x-2 i \hbar t, p)
$$

It is useful to employ the integral representation for the star-product (142) to rewrite the last expression as

$$
\begin{gathered}
\mathcal{A}_{2}=-a_{d} b_{d}^{-2} \int_{C} d \lambda \tilde{F}(\lambda) \int d x d p d x_{1} d p_{1} d x_{2} d p_{2} e^{\lambda p^{2}} \int_{0}^{\lambda} d \tau_{1} \int_{0}^{\tau_{1}} d t \\
\exp \left(\frac{2 i}{\hbar}\left[\left(x-x_{2}\right) p_{1}+\left(x_{1}-x\right) p_{2}+\left(x_{2}-x_{1}\right) p\right]\right) h\left(x_{1}, p_{1}\right) h\left(x_{2}-2 i \hbar t p_{2}, p_{2}\right),
\end{gathered}
$$

or, making shift of the integration variables $x_{2} \rightarrow x_{2}+2 i \hbar t p_{2}$,

$$
\begin{gathered}
\mathcal{A}_{2}=-a_{d} b_{d}^{-2} \int_{C} d \lambda \tilde{F}(\lambda) \int d x d p d x_{1} d p_{1} d x_{2} d p_{2} e^{\lambda p^{2}} \int_{0}^{\lambda} d \tau_{1} \int_{0}^{\tau_{1}} d t \\
\exp \left(\frac{2 i}{\hbar}\left[\left(x-x_{2}\right) p_{1}+\left(x_{1}-x\right) p_{2}+\left(x_{2}-x_{1}\right) p-2 i \hbar t p_{2}\left(p_{1}-p\right)\right]\right) h\left(x_{1}, p_{1}\right) h\left(x_{2}, p_{2}\right) .
\end{gathered}
$$

The next step is rewriting the integral by $\lambda, \tau_{1}, t$ using the formulae

$$
\begin{gathered}
\int_{0}^{\lambda} d \tau_{1} \int_{0}^{\tau_{1}} d t e^{t \sigma}=\frac{1}{\sigma}\left(\frac{1}{\sigma}\left(e^{\lambda \sigma}-1\right)-\lambda\right) \\
\int_{C} d \lambda \tilde{F}(\lambda) e^{\lambda \rho} \frac{1}{\sigma}\left(\frac{1}{\sigma}\left(e^{\lambda \sigma}-1\right)-\lambda\right)= \\
=\int_{0}^{1} d \alpha \int_{0}^{1} d \beta \alpha F^{\prime \prime}(\alpha \beta \sigma-\rho)
\end{gathered}
$$

to get

$$
\begin{gathered}
\mathcal{A}_{2}=-a_{d} b_{d}^{-2} \int_{0}^{1} d \alpha \int_{0}^{1} d \beta \alpha \int d x d p d x_{1} d p_{1} d x_{2} d p_{2} \delta^{\prime}(\alpha \beta \sigma-\rho) \\
\exp \left(\frac{2 i}{\hbar}\left[\left(x-x_{2}\right) p_{1}+\left(x_{1}-x\right) p_{2}+\left(x_{2}-x_{1}\right) p\right]\right) h\left(x_{1}, p_{1}\right) h\left(x_{2}, p_{2}\right),
\end{gathered}
$$

where

$$
\sigma=-4\left(p-p_{1}\right) p_{2} \quad, \quad \rho=-p^{2} .
$$

The integration over $x, p_{2}$ is elementary, with the result

$$
\begin{gathered}
\mathcal{A}_{2}=-a_{d} b_{d}^{-1} \int_{0}^{1} d \alpha \int_{0}^{1} d \beta \alpha \int d p d x_{1} d p_{1} d x_{2} \delta^{\prime}\left(4\left(p-p_{1}\right) p_{1} \alpha \beta-p^{2}\right) \\
\exp \left[\frac{2 i}{\hbar}\left(x_{1}-x_{2}\right)\left(p_{1}-p\right)\right] h\left(x_{1}, p_{1}\right) h\left(x_{2}, p_{1}\right),
\end{gathered}
$$

Instead of calculating the quadratic action

$$
\mathcal{A}_{P}[h]=\sum_{k=0, k^{\prime}=0}^{\infty} \int d^{d} x h^{m_{1} \ldots m_{k}}(x) P_{\left\{m_{1} \ldots m_{k} \mid n_{1} \ldots n_{k^{\prime}}\right\}}\left(\partial_{l}\right) h^{n_{1} \ldots n_{k^{\prime}}}(x),
$$


itself it is worth calculating the function of two vector variables

$$
P\left(q, q^{\prime}\right)=\sum_{k=0, k^{\prime}=0}^{\infty} \frac{1}{k ! k^{\prime} !} q^{n_{1}} \ldots q^{n_{k^{\prime}}} q^{\prime m_{1}} \ldots q^{\prime m_{k}} P_{\left\{m_{1} \ldots m_{k} \mid n_{1} \ldots n_{k^{\prime}}\right\}}\left(\partial_{l}\right)
$$

with the coefficients depending on $\partial_{l}$.

Lemma 1 If $\mathcal{A}_{2}$ is given by the expression

$$
\mathcal{A}_{P}[h]=\int d^{d} x_{1} d^{d} x_{2} d^{d} p f\left(\frac{x_{1}-x_{2}}{2}, p\right) h\left(x_{1}, p\right) h\left(x_{2}, p\right),
$$

then

$$
P\left(q, q^{\prime}, \partial\right)=2^{d} \int d^{d} r d^{d} p f(r, p) e^{\left(q^{m}+q^{\prime m}\right) p_{m}-2 r^{m} \partial_{m}}
$$

The proof is by direct calculation.

Now we are ready to calculate the generating function $P\left(q, q^{\prime}, \partial\right)$. According to (309) and (312,313), the expression for generating function reads

$$
\begin{gathered}
P\left(q, q^{\prime}, \partial\right)=-2^{d} a_{d} b_{d}^{-1} \int_{0}^{1} d \alpha \int_{0}^{1} d \beta \alpha \int d r d p d p_{1} \\
\delta^{\prime}\left(4\left(p-p_{1}\right) p_{1} \alpha \beta-p^{2}\right) \exp \left(\frac{4 i}{\hbar}\left[r\left(p_{1}-p\right)\right]+\left(q+q^{\prime}\right) p_{1}-2 r \partial\right) .
\end{gathered}
$$

Before doing integrals over $r$ etc. it is useful to make the shift of the integration variables

$$
p \rightarrow \Pi=p-2 p_{1} \alpha \beta
$$

and change the variables in the $\alpha, \beta$-sector as

$$
\eta=1-2 \alpha \beta, \alpha=\alpha
$$

to get

$$
\begin{gathered}
P\left(q, q^{\prime}, \partial\right)=-\frac{1}{2} 2^{d} a_{d} b_{d}^{-1} \int_{\Sigma} d \alpha d \eta \int d r d \Pi d p_{1} \\
\delta^{\prime}\left(p_{1}^{2}\left(\eta^{2}-1\right)-\Pi^{2}\right) \exp \left[\frac{4 i}{\hbar}\left(r\left(p_{1} \eta-\Pi\right)+\left(q+q^{\prime}\right) p_{1}-2 r \partial\right],\right.
\end{gathered}
$$

where the domain of integration $\Sigma$ in the $(\eta, \alpha)$ plane is the triangle with vertices $(1,0),(1,1)$, $(-1,1)$. Now perform elementary integration by $r, \Pi$ with the result

$$
\begin{gathered}
P\left(q, q^{\prime}, \partial\right)=-\frac{a_{d}}{2} \int_{\Sigma} d \alpha d \eta \int d p_{1} \\
\delta^{\prime}\left(\left(1-\eta^{2}\right) n^{2}-\left(p_{1}-i \eta n\right)^{2}+\right) e^{f p_{1}},
\end{gathered}
$$

where

$$
n=-\frac{\hbar}{2} \partial, f=q+q^{\prime} .
$$


After shift of the integration variables $p \rightarrow p+i \eta n$, the last integral takes the form

$$
\begin{gathered}
P\left(q, q^{\prime}, \partial\right)=-\frac{a_{d}}{2} \int_{\Sigma} d \alpha d \eta \int d p_{1} \\
\delta^{\prime}\left(\left(1-\eta^{2}\right) n^{2}-p_{1}^{2}\right) e^{f p_{1}} e^{i \eta n f}
\end{gathered}
$$

The remaining integral over $p_{1}$ is calculated easily as it is expressed via generating function $G_{d}^{(2)}$ (554,564),

$$
G_{d}^{(2)}(l)=\int d^{d} p \delta^{\prime}\left(M^{2}-p^{2}\right) e^{i p_{m} l^{m}}=2^{\frac{d}{2}-2} \pi^{\frac{d}{2}} M^{\frac{d}{2}-2} l^{-\frac{d}{2}+2} J_{\frac{d}{2}-2}(\mu l) .
$$

Combining two last formulae one gets

$$
\begin{gathered}
P\left(q, q^{\prime}, \partial\right)=-\frac{a_{d}}{2} 2^{\frac{d}{2}-2} \pi^{\frac{d}{2}} \\
\int_{\Sigma} d \alpha d \eta e^{i \eta n f}\left(n \sqrt{1-\eta^{2}}\right)^{\frac{d}{2}-2} J_{\frac{d}{2}-2}\left( \pm i|f||n| \sqrt{1-\eta^{2}}\right)
\end{gathered}
$$

One easily checks that the \pm -ambiguity in the separate multipliers of the last formula cancels for the whole expression.

Now integrate over $\alpha, \eta$. Note that the $\eta$-odd part of the last expression is purely imaginary and therefore it represents a total-derivative term (the associated contribution to the quadratic action gives operator of odd order in derivatives) which arose just because despite we have started from the purely real expression (288), after that we did some integrations by parts (we have used the cyclic property of the trace operation after the Eq. (200)).

In summary, $\eta$-odd term should be omitted and we are interested only in $\eta$-even contribution. But then the domain of integration may be supplemented (of course, after multiplying the total expression by $\frac{1}{2}$ ) by its image under $\eta \rightarrow-\eta$ reflection to get the rectangle with vertices $(0,1),(1,1),(1,-1),(0,-1)$. Then the integration by $\alpha$ gives the factor 1 as the integrand does not depend on $\alpha$, while for $\eta$ it is worth making the substitution

$$
\eta=\sin \theta, \quad \theta \in\left[-\frac{\pi}{2} ; \frac{\pi}{2}\right]
$$

The integral takes the form (wherein we have accounted that the integrand is even w.r.t. $\theta \rightarrow-\theta)$

$$
\begin{gathered}
P\left(q, q^{\prime}, \partial\right)=-\frac{a_{d}}{2} 2^{\frac{d}{2}-2} \pi^{\frac{d}{2}} \\
( \pm i f)^{2-\frac{d}{2}} \int_{0}^{\frac{\pi}{2}} d \theta \cos \theta e^{i \sin \theta n f}(n \cos \theta)^{\frac{d}{2}-2} J_{\frac{d}{2}-2}( \pm i|f||n| \cos \theta)
\end{gathered}
$$

Remarkably, the last integral by $\theta$ is calculated explicitly due to the existence of the special family of identities for Bessel's functions (570). Taking into account the identity

$$
J_{-\frac{1}{2}}(z)=\sqrt{\frac{2}{\pi z}} \cos z
$$


the integral (324) fits the identity (570) under the choice $q=\frac{1}{2}, p=\frac{d-3}{2}$. For the validity of the formula $(570)$ it is necessary $p \geq 0$ that excludes the case $d=2$, but this case is already excluded from our consideration as in $d=2 H=-p^{2}$ is not a vacuum.

The final result is $(d>2)$

$$
\begin{gathered}
P\left(q, q^{\prime}, \partial\right)=\kappa_{d}\left(n^{2}\right)^{\frac{d}{2}-2}\left(\sqrt{-n^{2} f^{2}+(n f)^{2}}\right)^{-\frac{d-3}{2}} J_{\frac{d-3}{2}}\left(\sqrt{-n^{2} f^{2}+(n f)^{2}}\right) \\
n=-\frac{\hbar}{2} \partial, f=q+q^{\prime}
\end{gathered}
$$

where

$$
\kappa_{d}=-2^{-\frac{d+7}{2}} \pi^{\frac{1-d}{2}} \hbar^{-d}
$$

No ambiguity in sign comes from the square root terms, as the expression $z^{-\nu} J_{\nu}(z)$ contains only even powers of $z$.

Let us comment on the emergence of the non-integer powers of $\square$ in odd dimensions. From the first glance it seems contradictory to our previous considerations as we are just calculating power series in derivatives in the framework of the semiclassical expansion. These apparent problems have arisen because we have used integral formula for the starproduct and then many times integrated by parts neglecting boundary terms. And the quadratic action corresponding to generating function (326) in odd dimensions is just a total derivative, if one defines nonlocal operators $\square^{\frac{k}{2}}, k=1,3,5, \ldots$ in such a way they are antisymmetric:

$$
\int d^{d} x A \square^{\frac{k}{2}} B=-\int d^{d} x B \square^{\frac{k}{2}} A+\text { boundary terms. }
$$

Hereafter we suppose this choice is made. So in odd dimensions the quadratic action vanishes.

\subsection{Spin decomposition}

As shown in [56] and in Sec. (3.1), conformal higher spin model of integer spin $s$ is characterized by the generating function

$$
\begin{gathered}
P_{\mu}^{(s)}\left(q, q^{\prime}, \partial\right)=\square^{\frac{d}{2}-2}\left(\rho \rho^{\prime}\right)^{-\frac{d-3}{2}} J_{s+\frac{d-3}{2}}(\mu \rho) J_{s+\frac{d-3}{2}}\left(\mu \rho^{\prime}\right) \mathcal{C}_{s}^{\frac{d-3}{2}}\left(\frac{\tau}{\rho \rho^{\prime}}\right), \\
\rho^{2}=\square q^{2}-(q \partial)^{2} ; \rho^{\prime 2}=\square q_{\perp}^{\prime 2}=\square q^{\prime 2}-\left(q^{\prime} \partial\right)^{2} \\
\tau=\square\left(q q^{\prime}\right)-(q \partial)\left(q^{\prime} \partial\right) .
\end{gathered}
$$

$\mu$ is a real operator, $\mu=\mu(\square)$. According to the theorem of Sec. (4.4), just calculated quadratic theory, characterized by the generating function (326) should break up as a 
sum of deformed conformal higher spin theories(83) with all spins $s=0,1,2,3, \ldots$, with deformation parameter

$$
\mu^{2}=-\frac{\hbar^{2}}{4} \Rightarrow \frac{\mu}{\sqrt{\square}}= \pm \frac{i \hbar}{2}
$$

while direct calculation should give specific values for the coefficients of the sum. Let us show by direct calculation this is really the case. To this end, note that the argument of the Bessel's function in (326) may be rewritten as

$$
\begin{gathered}
-n^{2} f^{2}+(n f)^{2}=-\frac{\hbar^{2}}{4}\left(\rho^{2}+\rho^{\prime 2}+2 \tau\right)=-\frac{\hbar^{2}}{4}\left(\rho^{2}+\rho^{\prime 2}+2 \rho \rho^{\prime} \cos \varphi\right) \\
\cos \varphi=\frac{\tau}{\rho \rho^{\prime}} .
\end{gathered}
$$

The last row is justified as for Euclidean vectors $q, q^{\prime}, \partial$ one has

$$
\tau=\square\left(q_{\perp} q_{\perp}^{\prime}\right) \leq \rho \rho^{\prime}=\square\left|q_{\perp}\right|\left|q_{\perp}^{\prime}\right|
$$

where $q_{\perp}$ stands for the $\partial$-transverse projection of $q$. The identity for Bessel's functions (571) allows one to decompose the generating function of the quadratic higher spin action (326) as follows

$$
\begin{gathered}
P\left(q, q^{\prime}, \partial\right)=\kappa_{d} 2^{\frac{d-3}{2}} \Gamma\left(\frac{d-3}{2}\right) \\
\left(n^{2}\right)^{\frac{d}{2}-2} \sum_{s=0}^{\infty}(-)^{s}\left(s+\frac{d-3}{2}\right)\left( \pm \frac{i \hbar}{2} \rho\right)^{\frac{d-3}{2}}\left( \pm \frac{i \hbar}{2} \rho^{\prime}\right)^{\frac{d-3}{2}} J_{s+\frac{d-3}{2}}\left( \pm \frac{i \hbar}{2} \rho\right) J_{s+\frac{d-3}{2}}\left( \pm \frac{i \hbar}{2} \rho^{\prime}\right) \mathcal{C}_{s}^{\frac{d-3}{2}}\left(\frac{\tau}{\rho \rho^{\prime}}\right)= \\
=2^{\frac{d-3}{2}}\left( \pm \frac{i \hbar}{2}\right)^{d-3}\left(\frac{\hbar}{2}\right)^{d-4} \kappa_{d} \sum_{k=0}^{\infty}(-)^{s} \Gamma\left(s+\frac{d-1}{2}\right) P_{ \pm \frac{i \hbar}{2}}^{(s)}\left(q, q^{\prime}, \partial\right)= \\
-2^{-1-d} \pi^{\frac{1-d}{2}}\left( \pm \frac{i \hbar}{2}\right)^{d-3} \hbar^{-4} \sum_{k=0}^{\infty}(-)^{s} \Gamma\left(s+\frac{d-1}{2}\right) P_{ \pm \frac{i \hbar}{2}}^{(s)}\left(q, q^{\prime}, \partial\right)
\end{gathered}
$$

As above, note that the \pm -ambiguity disappears as all expressions are even either w.r.t. $\rho$ or $\rho^{\prime}$.

We have shown that the quadratic action is an infinite sum of conformal higher spin models of all integer spins $s=0,1,2,3, \ldots$ More precisely, the quadratic action, corresponding to the generating function (334) is rewritten in terms of undressed variables $\varphi_{s}$ introduced in Sec. (3.2) as a sum of conformal higher spin actions described in Sec. (2.2.1). This is the corollary of the paper. 


\subsection{Calculation of the cubic part of the action}

We start with the expression for the cubic part of the action $\mathcal{A}_{3}$ (293), which we rewrite using cyclic property of the trace and the equations

$$
\bar{H}=-p^{2}, e^{\lambda p^{2} *}=e^{\lambda p^{2}}
$$

in the form

$$
\begin{gathered}
\mathcal{A}_{3}=-\frac{1}{2} a_{d} \int_{C} d \lambda \tilde{F}(\lambda) \int d x d p e^{\lambda p^{2}} \\
\int_{0}^{\lambda} d \tau_{1} \int_{0}^{\tau_{1}} d \tau_{2} \int_{0}^{\tau_{2}} d \tau_{3} h * e^{\left(\tau_{1}-\tau_{2}\right) p^{2}} * h * e^{-\left(\tau_{1}-\tau_{2}\right) p^{2}} e^{\left(\tau_{1}-\tau_{3}\right) p^{2}} * h * e^{-\left(\tau_{1}-\tau_{3}\right) p^{2}} .
\end{gathered}
$$

Making change of variables

$$
\begin{aligned}
& t_{1}=\tau_{1}-\tau_{3}, \quad t_{2}=\tau_{1}-\tau_{2} \\
& t_{2} \leq t_{1} \leq \tau_{1} \quad, \quad 0 \leq \tau_{2} \leq \tau_{1}
\end{aligned}
$$

and using

$$
e^{\lambda p^{2} *} * h(x, p) * e^{-\lambda p^{2} *}=h(x-2 i \hbar \lambda p, p),
$$

the cubic action is rewritten as

$$
\begin{gathered}
\mathcal{A}_{3}=-\frac{1}{2} a_{d} \int_{C} d \lambda \tilde{F}(\lambda) \int_{\Sigma} d \tau_{1} d t_{1} d t_{2} \int d x d p e^{\lambda p^{2}} \\
\int_{0}^{\lambda} d \tau_{1} \int_{0}^{\tau_{1}} d t_{2} \int_{t_{2}}^{\tau_{1}} d t_{1} h(x, p) * h\left(x-2 i \hbar t_{2} p, p\right) * h\left(x-2 i \hbar t_{1} p, p\right),
\end{gathered}
$$

where integration over $\tau_{1}, t_{1}, t_{2}$ goes over the domain $\Sigma$ specified by the second row of the Eq. (337) and the condition $\tau_{1} \in[0,1]$.

Employing integral representation for the star-product (142) and making some simple redefinition of integration variables one rewrites the last expression as

$$
\begin{aligned}
& \mathcal{A}_{3}=-\frac{1}{2} a_{d} b_{d}^{-2} \int_{C} d \lambda \tilde{F}(\lambda) \int_{\Sigma} d \tau_{1} d t_{1} d t_{2} \int d x d p d x_{1} d p_{1} d x_{2} d p_{2} d x_{1}^{\prime} d p_{1}^{\prime} d x_{2}^{\prime} d p_{2}^{\prime} e^{\lambda p^{2}} \\
& \exp \left(\frac{2 i}{\hbar}\left[\left(x-x_{2}^{\prime}\right) p_{1}^{\prime}+\left(x_{1}^{\prime}-x\right) p_{2}^{\prime}+\left(x_{2}^{\prime}-x_{1}^{\prime}\right) p+x_{2}^{\prime}-x_{2}\right) p_{1}+\left(x_{1}-x_{2}^{\prime}\right) p_{2}^{\prime}+\right. \\
&\left.\left.\quad+2 i \hbar\left(p_{1} p_{2}\left(t_{2}-t_{1}\right)+t_{1} p_{2} p_{2}^{\prime}-t_{2} p_{1} p_{2}^{\prime}\right)\right]\right) B\left(x_{1}^{\prime}, p_{1}^{\prime}\right) B\left(x_{1} p_{1}\right) B\left(x_{2}, p_{2}\right)
\end{aligned}
$$


Doing elementary integrations over $x, p, x_{2}^{\prime}, p_{2}^{\prime}$ one gets

$$
\begin{gathered}
\mathcal{A}_{3}=-\frac{1}{2} a_{d} \int_{C} d \lambda \tilde{F}(\lambda) \int_{\Sigma} d \tau_{1} d t_{1} d t_{2} \int d x_{1} d p_{1} d x_{2} d p_{2} d x_{1}^{\prime} d p_{1}^{\prime} e^{\lambda\left(p_{1}^{\prime}+p_{2}-p_{1}\right)^{2}} \\
\exp \left(\frac{2 i}{\hbar}\left[\left(p-p_{2}\right) x_{1}^{\prime}+p_{1}^{\prime}\left(x_{2}-x_{1}\right)+x_{1} p_{2}-x_{2} p_{1}+2 i \hbar\left(\left(p_{1}^{\prime}-p_{1}\right) p_{2} t_{1}+\left(p_{2}-p_{1}^{\prime}\right) p_{1} t_{2}\right)\right]\right) \\
B\left(x_{1}^{\prime}, p_{1}^{\prime}\right) B\left(x_{1} p_{1}\right) B\left(x_{2}, p_{2}\right) .
\end{gathered}
$$

To integrate by $\lambda$ one has to make use of the equality

$$
\begin{gathered}
\int d \lambda \tilde{F}(\lambda) \int_{0}^{\lambda} d \tau_{1} \int_{0}^{\tau_{1}} d t_{2} \int_{t_{2}}^{\tau_{1}} e^{-\lambda \sigma_{3}+t_{1} \sigma_{1}+t_{2} \sigma_{2}}= \\
-\int_{0}^{1} d \alpha \alpha^{2} \int_{0}^{1} d \beta \int_{\beta}^{1} d \gamma F^{\prime \prime \prime}\left(\sigma_{3}-\left(\gamma \sigma_{1}+\beta \sigma_{2}\right) \alpha\right),
\end{gathered}
$$

with

$$
\sigma_{1}=-\left(p_{1}^{\prime}-p_{1}+p_{2}\right)^{2}, \sigma_{2}=-4\left(p_{1}^{\prime}-p_{1}\right) p_{2} \quad, \quad \sigma_{3}=-4 p_{2}\left(p_{2}-p_{1}^{\prime}\right)
$$

to get

$$
\begin{gathered}
\mathcal{A}_{3}=-\frac{1}{2} a_{d} \int_{0}^{1} d \alpha \alpha^{2} \int_{0}^{1} d \beta \int_{\beta}^{1} d \gamma \int d x_{1} d p_{1} d x_{2} d p_{2} d x_{1}^{\prime} d p_{1}^{\prime} \delta^{\prime \prime}\left(\sigma_{3}-\left(\gamma \sigma_{1}+\beta \sigma_{2}\right) \alpha\right) \\
e^{\left.\frac{2 i}{\hbar}\left[\left(p_{1}-p_{2}\right) x_{1}^{\prime}\right]+\left(x_{2}-x_{1}\right) p_{1}^{\prime}+x_{1} p_{2}-x_{2} p_{1}\right]} B\left(x_{1}^{\prime}, p_{1}^{\prime}\right) B\left(x_{1} p_{1}\right) B\left(x_{2}, p_{2}\right) .
\end{gathered}
$$

To exhibit the structure of the cubic action it is worth calculating not the action itself but a special generating function which encodes the complete structure of the cubic action.

Let

$$
\mathcal{A}_{3}=\int d x \sum_{s_{1}, s_{2}, s_{3}, k_{1}, k_{2}, k_{3}=0}^{\infty} P_{a_{1}\left(s_{1}\right) a_{2}\left(s_{2}\right) a_{3}\left(s_{3}\right)}^{b_{1}\left(k_{1}\right) b_{2}\left(k_{2}\right) b_{3}\left(k_{3}\right)} h_{, b_{1}\left(k_{1}\right)}^{a_{1}\left(s_{1}\right)}(x) h_{, b_{2}\left(k_{2}\right)}^{a_{2}\left(s_{2}\right)}(x) h_{, b_{3}\left(k_{3}\right)}^{a_{3}\left(s_{3}\right)}(x)
$$

be general cubic action (commas denote derivatives w.r.t. $x^{m}$ ). Generating function is the function of six vector variables $b_{1}^{m}, b_{2}^{m}, b_{3}^{m}, d_{1 m}, d_{2 m}, d_{3 m}$ of the form

$$
\begin{aligned}
& P_{3}=\sum_{s_{1}, s_{2}, s_{3}, k_{1}, k_{2}, k_{3}=0}^{\infty} \frac{1}{s_{1} ! s_{2} ! s_{3} !} P_{a_{1}\left(s_{1}\right) a_{2}\left(s_{2}\right) a_{3}\left(s_{3}\right)}^{b_{1}\left(k_{1}\right) b_{2}\left(k_{2}\right) b_{3}\left(k_{3}\right)} \\
& b_{1}^{a_{1}} \ldots b_{1}^{a_{1 s_{1}}} b_{2}^{a_{2}} \ldots b_{2}^{a_{2 s_{2}}} b_{3}^{a_{3}} \ldots b_{3}^{a_{3 s_{3}}} d_{1 b_{1}} \ldots d_{1 b_{1 k_{1}}} d_{2 b_{2}} \ldots d_{2 b_{2 k_{2}}} d_{3 b_{1}} \ldots d_{3 b_{1 k_{1}}} .
\end{aligned}
$$

Lemma 2 Let the cubic action is given by the formula

$\mathcal{A}_{3}=\int d x_{1} d x_{2} d x_{3} d p_{1} d p_{2} d p_{3} f\left(Q_{2}-Q_{1}, Q_{3}-Q_{2}, Q_{1}-Q_{3}\right) h\left(Y+Q_{1}\right) h\left(Y+Q_{2}\right) h\left(Y+Q_{3}\right)$ 
where vector variables $Y, Q_{1,2,3}$ are defined as follows

$$
x_{1,2,3}=Y+Q_{1,2,3}, Q_{1}+Q_{2}+Q_{3}=0 .
$$

Then the generating function $P_{3}$ is

$$
P_{3}=3^{-d} \int d Q_{1} d Q_{2} f\left(Q_{2}-Q_{1},-Q_{1}-2 Q_{2}, 2 Q_{1}+Q_{2}\right) e^{Q_{1}\left(d_{2}-d_{1}\right)+Q_{2}\left(d_{3}-d_{1}\right)+p_{1} b_{1}+p_{2} b_{2}+p_{3} b_{3}} .
$$

The proof is by direct calculation. Combining this lemma with the expression (8.4) one finds

$$
\begin{gathered}
P_{3}=-\frac{1}{2} 3^{-d} a_{d} \int_{0}^{1} d \alpha \alpha^{2} \int_{0}^{1} d \beta \int_{\beta}^{1} d \gamma \int d Q_{1} d Q_{2} d p_{1} d p_{2} d p_{1}^{\prime} \delta^{\prime \prime}\left(\sigma_{3}-\left(\gamma \sigma_{1}+\beta \sigma_{2}\right) \alpha\right) \\
\exp \left(\frac { 2 i } { \hbar } \left[Q _ { 1 } \left(-p_{1}+2 p_{2}-p_{1}^{\prime}+\frac{\hbar}{2 i}\left(d_{2}-d_{1}\right)+Q_{2}\left(p_{2}-2 p_{1}+p_{1}^{\prime}+\frac{\hbar}{2 i}\left(d_{3}-d_{1}\right)\right]\right.\right.\right. \\
\left.+p_{1} b_{1}+p_{2} b_{2}+p_{1}^{\prime} b_{3}\right)
\end{gathered}
$$

Doing elementary integration by $Q_{1}, Q_{2}, p_{1}, p_{2}$ one gets

$$
\begin{gathered}
P_{3}=-\frac{1}{2} 3^{-d} a_{d} b_{d}^{2} \int_{0}^{1} d \alpha \alpha^{2} \int_{0}^{1} d \beta \int_{\beta}^{1} d \gamma \int d p_{1}^{\prime} \delta^{\prime \prime}\left(\sigma_{3}-\left(\gamma \sigma_{1}+\beta \sigma_{2}\right) \alpha\right) \\
\exp \left[\left(p_{1}^{\prime}+\frac{1}{3}\left(2 n_{2}-n_{1}\right)\right) b_{1}+\left(p_{1}^{\prime}+\frac{1}{3}\left(n_{2}-2 n_{1}\right)\right) b_{2}+p_{1}^{\prime} b_{3}\right],
\end{gathered}
$$

where

$$
\begin{gathered}
n_{1}=\frac{\hbar}{2 i}\left(d_{2}-d_{1}\right), n_{2}=\frac{\hbar}{2 i}\left(d_{3}-d_{1}\right) \\
\sigma_{1}=\frac{4}{3}\left(p_{1}^{\prime}+\frac{1}{3}\left(n_{2}-2 n_{1}\right)\right)\left(2 n_{2}-n_{1}\right), \sigma_{2}=-\frac{4}{3}\left(p_{1}^{\prime}+\frac{1}{3}\left(2 n_{2}-n_{1}\right)\right)\left(n_{2}-2 n_{1}\right) \\
\sigma_{3}=-\left(p_{1}^{\prime}-\frac{1}{3}\left(n_{2}+n_{1}\right)\right)^{2} .
\end{gathered}
$$

The argument of $\delta^{\prime \prime}$ is rewritten as

$$
\begin{gathered}
\sigma_{3}-\left(\gamma \sigma_{1}+\beta \sigma_{2}\right) \alpha=-\left(p_{1}^{\prime}+\delta_{1} n_{1}+\delta_{2} n_{2}\right)^{2}+A^{2}\left(n_{1}, n_{2} \mid \delta_{1}, \delta_{2}\right), \\
A^{2}=\left(\delta_{1} n_{1}+\delta_{2} n_{2}\right)^{2}-\frac{2}{9}\left(\delta_{2}-\delta_{1}\right)\left(n_{2}-2 n_{1}\right)\left(2 n_{2}-n_{1}\right)-\frac{1}{9}\left(n_{1}+n_{2}\right)^{2} \\
\delta_{1}=\frac{1}{3}(-1+\alpha(4 \beta-2 \gamma)), \delta_{2}=\frac{1}{3}(-1+\alpha(4 \gamma-2 \beta)) .
\end{gathered}
$$

Making change of variables $\Pi=p_{1}^{\prime}+\delta_{1} n_{1}+\delta_{1} n_{2}$ one gets

$$
\begin{gathered}
P_{3}=-\frac{1}{2} 3^{-d} a_{d} b_{d}^{2} \int_{0}^{1} d \alpha \alpha^{2} \int_{0}^{1} d \beta \int_{\beta}^{1} d \gamma \int d \Pi \delta^{\prime \prime}\left(-\Pi^{2}+A\left(n_{1}, n_{2} \mid \delta_{1}, \delta_{2}\right)\right) \\
\exp \left(\Pi\left(b_{1}+b_{2}+b_{3}\right)-\left(\delta_{1} n_{1}+\delta_{1} n_{2}\right)\left(b_{1}+b_{1}+b_{3}\right)+\frac{1}{3}\left(2 n_{2}-n_{1}\right) b_{1}+\frac{1}{3}\left(n_{2}-2 n_{1}\right) b_{2}\right)
\end{gathered}
$$


Just like in the calculation of the quadratic action, the integration over momenta is performed easily as it is given by the generating function $G_{d}^{(3)}$ (see 554,564):

$$
G_{d}^{(3)}(l)=\int d^{d} p \delta^{\prime \prime}\left(M^{2}-p^{2}\right) e^{i p_{m} l^{m}}=2^{\frac{d}{2}-3} \pi^{\frac{d}{2}} M^{\frac{d}{2}-3} l^{-\frac{d}{2}+3} J_{\frac{d}{2}-3}(\mu l)
$$

Combining the last formula with the expression for the generating function (355) one arrives at the formula (wherein $f=b_{1}+b_{2}+b_{3}$ )

$$
\begin{gathered}
P_{3}=-2^{-\frac{d}{2}-4} \pi^{\frac{3 d}{2}} 3^{-d} \hbar^{d} e^{\frac{1}{3}\left(2 n_{2}-n_{1}\right) b_{1}+\frac{1}{3}\left(n_{2}-2 n_{1}\right) b_{2}}(f)^{3-\frac{d}{2}} \int_{\Sigma} d \alpha d \delta_{1} d \delta_{2} \\
e^{-\left(\delta_{1} n_{1}+\delta_{2} n_{2}\right) f} A\left(n_{1}, n_{2} \mid \delta_{1}, \delta_{2}\right)^{\frac{d}{2}-3} J_{\frac{d}{2}-3}\left(f A\left(n_{1}, n_{2} \mid \delta_{1}, \delta_{2}\right)\right),
\end{gathered}
$$

where the integration by $\alpha, \delta_{1}, \delta_{2}$ goes over the domain $\Sigma$ in the three-dimensional space with coordinates $\alpha, \delta_{1}+\delta_{2}+\frac{2}{3}, \delta_{2}-\delta_{1}$, where each plane with fixed $\alpha \in[0,1]$ is a triangle with vertices $\alpha(1,0,0), \alpha(1,1,1), \alpha(1,2,1)$. The integration may be performed by decomposing analytic function $z^{\frac{d}{2}-3} J_{\frac{d}{2}-3}(z)$ in power series in $z$ then each term of the decomposition of the integrand is a polynomial in $A\left(n_{1}, n_{2} \mid \delta_{1}, \delta_{2}\right)$ and the integral runs over the compact domain $\Sigma$.

Let us briefly discuss the structure of cubic vertices. It reproduces general structure anticipated in the beginning of this section,

$$
A_{3}[h] \sim \int d^{d} x \sum_{s_{1}, s_{2}, s_{3}=0}^{\infty} \partial^{d-6+s_{1}+s_{2}+s_{3}} h_{s_{1}} \ldots h_{s_{3}},
$$

and the exact expression (357) just gives the special values to the coefficients of separate terms in the last formula.

Let us comment of the procedure of finding interactions of original conformal higher spin fields, described by traceless symmetric tensors. We saw in Sec. (3.2) that the quadratic action of deformed conformal higher spin theories is expressed via "undressed fields $\phi_{s}(x), s=0,1,2,3, \ldots$ (96), (98) related to $h_{s}$ by the invertible field redefinition (104),(105) and that only traceless parts of undressed fields $\varphi_{s}$ contribute the action. Thus, at the quadratic level the action is entirely expressed in terms of $\varphi_{s}$. The cubic action we just have calculated depends, however, on $h_{s}$ or, what is the same, on all $\phi_{s}$, i.e. on $\varphi_{s}$ and on trace parts of $\phi_{s}$. This means that in order to formulate the cubic action one has to add all the trace parts of $\phi_{s}$ to original traceless fields $\varphi_{s}$ the quadratic action is build of. Thus from a first glance it seems as if at nonlinear level one has to introduce additional degrees of freedom that is dangerous, because variation w.r.t. these additional fields would give new equations of motion and make dynamics inconsistent in general. However this is of course not the case. First of all let us note that the decoupling of the traces of $\phi_{s}$ is controlled at the quadratic level by the hyper-Weyl gauge invariance, 
which is being smoothly deformed to nonlinear level, the latter property is in the core of our construction. Thus one makes sure analogous decoupling phenomena will take place at the nonlinear level, and one just has to get which form this decoupling takes at the cubic order.

In fact it is easy to describe the decoupling of the traces at the cubic level, as according to the well-known statement (App. (A)), the cubic action is gauge-invariant w.r.t. linearized gauge transformations provided the fields obey linearized equations of motion. Therefore, if the fields satisfy the linearized equations of motion then traces of $\phi_{s}$ do decouple. This implies the traces of $\phi_{s}$ enter the cubic action only as a part of linearized equations of motion. This phenomenon, and analogous ones which are nothing but the consequences of the full gauge invariance $\delta H=\omega^{\dagger} * H+H * \omega$, make it consistent the procedure of solving full nonlinear equations perturbatively by representing dynamical fields as a formal power series in $e, h=h_{0}+e h_{1}+e^{2} h_{2}+\ldots$ and solving the nonlinear equations order by order in $e$. For instance, up to the cubic order one has, schematically,

$$
L_{2} h_{0}=0 ; L_{2} h_{1}=L_{3} h_{0} h_{0}
$$

where $L_{2}$ and $L_{3}$ are structures governing the quadratic and the cubic actions. From the first equation one gets that $h_{0}$ obeys free equations of motion, the latter are gauge invariant w.r.t. linearized gauge transformations that implies decoupling of the traces. By the same reasons, the traces of $h_{1}$ decouple in the second equation, while the "source" $L_{3} h_{0} h_{0}$ is gauge invariant w.r.t. linearized gauge transformations as $h_{0}$ is on-shell. To summarize, the traces of the fluctuation $h_{0}+e h_{1}$ do not contribute equations of motion up to cubic order, therefore, no dangerous phenomena arise. Due to analogous decoupling phenomena, the procedure of perturbative solution of the equations of motion will be always consistent and will give interactions of the infinite number of traceless tensors of all spins, each spin will enter the theory just once.

One more important issue is $d=3,4$ case and comparison of our results with results by Fradkin and Linetsky [14]. Although we do not pursue explicit comparison in this paper it is worth making a few comments. First of all note that in $d=3$ the action by Fradkin and Linetsky is just a Chern-Simons action which does not set a dynamical three-dimensional theory, in this sense our result that the quadratic part of the action of conformal higher spin theory in odd dimensions is a boundary term conforms with results of the authors of [14. In $d=4$ the authors investigated construction of the cubic interaction in a supersymmetric theory of conformal higher spin fields with spins $s=1, \frac{3}{2}, 2, \frac{5}{2}, 3, \frac{7}{2}, 4 \ldots$ and concluded there is no consistent interaction beyond cubic order until each spin enters the theory with infinite multiplicity. This looks different from our consideration as we 
have the theory of all spins, from zero to infinity, with multiplicity one. Leaving aside difference of approaches and difference of supersymmetric and purely boson treatment one notes that perhaps the main difference is the presence in our formulation of spin-0 field that is purely auxiliarym. At the quadratic level, it is zero on-shell while at higher levels $s=0$ field is expressed algebraically via the higher spin fields. Namely, employing schematic representation of the cubic interaction (358) one gets at the cubic order

$$
h_{0,0}(x)=0 ; h_{1,0}(x) \sim \sum_{s_{1}, s_{2}=1}^{\infty} \partial^{-2+s_{1}+s_{2}} h_{0, s_{1}} h_{0, s_{2}},
$$

where $h_{0, s}, h_{1, s}$ stand for the zero and the first order of the perturbative expansion for the component of rank $s$. It will give additional, "dynamical" vertices for higher spin fields in addition to those explicitly encoded in generating function (357). To summarize, we observe the problems with infinite multiplicities of all spins (and, of course, problems with all-orders interaction) encountered by Fradkin and Linetsky are avoided in our approach, which differs by the presence of the auxiliary spin-0 scalar.

\section{$9 \quad$ Free particles and conformal higher spin fields}

It is clear one may consider the united action

$$
\begin{gathered}
\mathcal{A}[H, \psi]=\mathcal{A}[H]+S_{H}[\psi]= \\
\operatorname{Tr} \theta(\hat{H})+\left(\frac{1}{2}<\psi|\hat{H}| \psi>+\frac{1}{2}(<\psi|\hat{H}| \psi>)^{*}\right),
\end{gathered}
$$

where $e$ is a coupling constant. In the classical limit, interaction with classical particle is achieved by adding the coupling

$$
S_{H}[x, p, \lambda]=\int d \tau\left\{p_{m} \dot{x}^{m}-\lambda H(x, p)\right\}
$$

Then the equations of motion read

$$
\begin{gathered}
\delta_{H} \mathcal{A}[H, \psi]=\delta(\hat{H})+e|\psi><\psi|=0 \\
\delta_{<\psi \mid} \mathcal{A}[H, \psi]=\hat{H} \mid \psi>=0 .
\end{gathered}
$$

The interpretation of the second equation is that $|\psi\rangle$ is a zero mode for $H$, while from the first equation one gets that $|\psi><\psi|$ is a projector to zero modes subspace. In fact, the second equation is the consequence of the first one. All this means that the zero modes subspace is one-dimensional. Thus, in the formal operator approach, the classical

\footnotetext{
${ }^{11}$ Besides, in the purely boson version of Fradkin and Linetsky's model there is no spin-1 Maxwell field.
} 
dynamics, described by the united action (361), just describes, for $e \neq 0$ the hamiltonians with one-dimensional normalizable zero modes subspace. In the case $e=0$ one gets the formal equation $\delta(\hat{H})=0$ which is can be interpreted as a condition to $\hat{H}$ not to admit normalizable zero modes in $\mathbf{L}^{2}$, this is discussed in more detail in App. (B).

Here it is also worth remarking that this interpretation is just a formal picture that may have a little to do with actual dynamics of conformal higher spin fields. On the other hand, in some cases this picture may provide an adequate description, therefore we find it useful to keep it in mind.

In $d>2$, the equations (363) possess Poincaré-invariant solution

$$
H=-p^{2}, \psi=0
$$

The perturbative decomposition of the action (361) around this vacuum describes interaction of massless complex scalar and the tower of real conformal higher spin fields of all integer spins. Note that in $d=4$ the conformal spin-0 scalar is an auxiliary field, while in $d=6$ it is the familiar real massless scalar, therefore there are in total three real massless scalars in this case. In the cubic order in fluctuations one gets the picture of sources built from the bilinear combinations of wave function interacting by means of exchange by conformal higher spin fields. Namely, the equations (363) are solved perturbatively, order by order in the coupling constant $e$, after representing the Hamiltonian $H(x, p)$ and the wave function $\psi(x)$ in series in $e$ as

$$
H=-p^{2}+\sum_{k=1}^{\infty} e^{k} h_{k}(x, p), \psi(x)=\sum_{k=0}^{\infty} e^{k} \psi_{k}(x) .
$$

Then in zero and the first order in $e$ one gets the equations of motion

$$
\begin{gathered}
\delta\left(p^{2}\right)=0 \\
\hat{p}^{2} \mid \psi_{0}>=0 \\
L \hat{h}_{1}=\left|\psi_{0}><\psi_{0}\right|,
\end{gathered}
$$

where $L$ is the wave operator for linearized equations of deformed conformal higher spin fields, calculated above. In the classical limit, one gets

$$
\begin{gathered}
\delta\left(p^{2}\right)=0 \\
L h_{1}(x, p)=\int d \tau \lambda(\tau) \delta(x-x(\tau), p-p(\tau)),
\end{gathered}
$$

where $x(\tau), p(\tau)$ is an on-shell trajectory, and $\delta(x, p)$ is $2 d$-dimensional $\delta$-function. The r.h.s. of the second row is nothing but the (relativistic) phase space density which thereby plays the role of a source for conformal higher spin fields. 
The interpretation of these equations is that there exists a source for conformal higher spin fields built from the bilinear combination of the on-shell wave function $|\psi\rangle$ (or, in the classical limit, the source is a functional of classical trajectories). After analytic continuation to Minkowski space, the equations $L h_{1}=\ldots$ describe radiation of conformal higher spin fields by the complex scalar field $\psi(x)$, or, in the classical limit, by the massless point particle, and their propagation through space-time according to free equations of motion.

After this equation is solved for $h_{1}$ in terms of $\psi(x)$ and some initial(boundary) data one can substitute the result back to the action (361) to get the first order interaction of the sources in the form

$$
S_{\text {int }}=e \int d^{d} x \psi_{0}^{*}(x) \hat{h}_{1} \psi_{0}(x),
$$

where $\psi_{0}(x)$ are on-shell, $\hat{p}^{2} \psi_{0}=0$. This action is gauge invariant w.r.t. linearized gauge transformations

$$
\delta h(x, p)=\omega(x, p)^{\dagger} * p^{2}+p^{2} * \omega(x, p),
$$

as $\hat{p}^{2} \psi(x)=0$. This invariance is nothing but the manifestation of the covariance of the coupling $S_{H}[\psi]=e<\psi|\hat{H}| \psi>$ in all orders in $e$. Indeed, expanding $S_{H}$ and gauge transformations (137) in $e$, one gets, in the lowest order in $e$, the invariance of $S_{\text {int }}$ w.r.t. linearized gauge transformations (369) provided $\psi$ is on-shell w.r.t. vacuum Hamiltonian. As we show in Sec. (11) the interaction (368) is an economic form of Noether interaction of the on-shell complex scalar field $\psi$ to the external gauge fields via "Noether currents one-forms" coupling, with (369) being the counterpart of abelian gauge transformations of linearized connections.

Note that in $d=4$ the linearized equation for the scalar fluctuation $h(x)$ is purely algebraic, while the corresponding current does not contain derivatives, so one gets the quartic interaction of the form $\sim\left(\int d^{d} x|\psi(x)|^{2}\right)^{2} . \quad s=1$ contribution in $d=4$ is a standard interaction of charged matter sources via Maxwell fields.

\section{Global Symmetries. Conformal algebra and its infinite-dimensional extension, conformal higher spin algebra. Higher spin currents}

This section presents an independent study of global symmetries of the conformal higher spin model. The global symmetries are gauge transformations that preserve the vacuum. The emergence of higher spin fields as background fields of the point particle allows 
one to describe the algebra of global symmetries in a simple fashion, as algebra of global symmetries of the particle. This way we describe all known conformal higher spin algebras (in $d>2$ ) as algebras of observables of massless particle in an arbitrary dimension, and besides we give definition of boson conformal higher spin algebras in an arbitrary dimension $d$.

General technique of symbols of operators we use allows us to derive a simple formula for all Noether currents of free complex scalar field for any wave operator, provided its global symmetries are known. In particular, in the case of the wave operator $\hat{H}=\hbar^{2} \square$ our formula reproduces, in another basis, the set of currents found in [12] by direct calculation.

But first of all we will show that the global symmetries of the vacuum $\hat{H}=\hbar^{2} \square$ contain the conformal algebra $s o(d-q+1, q+1)$ and that its action on undressed fluctuations $\varphi_{s}$ is diagonal w.r.t. spin $s$. This means we deal with standard conformal theory in any dimension $d>2$. Then we will pass to the next issues related to the infinite-dimensional extension of the conformal algebra to the conformal higher spin algebra.

\subsection{Undressing, Reconstruction, and Conformal invariance.}

Consider the field $f(x)$ taking values in a representation of the Lorentz algebra $s o(d-q, q)$ with generators $M_{a b}, a, b=0, \ldots, d-1$. Then one has the associated representation of the conformal algebra $s o(d-q+1, q+1)$ with generators

$$
\begin{gathered}
\tilde{P}_{a}=-\partial_{a}, \tilde{J}_{a b}=-\left(x_{a} \partial_{b}-x_{b} \partial_{a}\right)+M_{a b} \\
\tilde{D}=x \partial_{x}+\gamma, \tilde{K}_{a}=-\left(x^{2} \partial_{a}-2 x_{a} x \partial_{x}\right)-2 x^{b} M_{a b}-2 x_{a} \gamma,
\end{gathered}
$$

where $\gamma$ is an arbitrary constant, the conformal weight.

Consider the representation of the conformal group on the components of the Hamiltonian $H(x, p)$. By construction, it is given by the gauge transformations (209) which leave intact the vacuum Hamiltonian $\bar{H}=-p^{2}$,

$$
\begin{gathered}
\delta H(x, p)=\omega^{\dagger} * H+H * \omega, \omega=a+\frac{i}{\hbar} \epsilon \\
\epsilon=b^{a} P_{a}+b^{a b} J_{a b}+c^{a} K_{a}+c D \\
P_{a}=p_{a}, J_{a b}=x_{a} p_{b}-x_{b} p_{a}, D=-(x, p), K_{a}=x^{2} p_{a}-2 x_{a}(x, p), \\
a=-c+2 c^{a} x_{a},
\end{gathered}
$$

that is in accord with (47). These transformations exhaust global symmetries with $\epsilon$ linear in momenta, that is the subject of our analysis in this section. Using the formula (140) 
for the star-product, one derives

$$
\delta H(x, p)=\left[b^{a} \tilde{P}_{a}+b^{a b} \tilde{J}_{a b}+c^{a} \tilde{K}_{a}+c \tilde{D}+\frac{\hbar^{2}}{4} c^{a}\left(\partial_{p}^{2} \partial_{a}-2\left(\partial_{x} \partial_{p}\right) \delta_{a}\right)\right] H(x, p)
$$

where $\delta^{a}=\frac{\partial}{\partial p_{a}}$ and operators $\tilde{P}_{a}, \tilde{J}_{a b}, \tilde{K}_{a}, \tilde{D}$ map each rank-s subspace of $H$ into itself and act in this subspaces as the generators of the conformal algebra (370) with

$$
M_{a b}=-\left(p_{a} \delta_{b}-p_{b} \delta_{a}\right), \quad \gamma=2-p \partial_{p}
$$

One observes that the conventional tensor transformation laws (370) are deformed by the quantum correction, represented by the $\hbar^{3}$-term in (372). As a consequence, the conformal transformations mix fields of different spins. However, at the linearized level this mixing miraculously cancels after passing to the "undressed fields" of Sec. (3.2). Indeed, consider representation of the global symmetry in the space of fluctuation near the conformally flat vacuum $H=-p^{2}, h(x, p)$. It is given (see App. (A)) by the differential of the full global symmetry transformations (371) evaluated at the vacuum point. However, as the global transformations (371) are linear, their differential looks just the same, with $H \rightarrow h$ substitution,

$$
\delta h(x, p)=\omega^{\dagger} * h+h * \omega .
$$

As it is shown in Sec. (3.2), at the linearized level it is possible to set the undressed fields $\phi_{s}$ (98) traceless without loss of generality, this implies in turn that $h$ is traceless either (that may be easily seen by inspecting reconstruction map (105)),

$$
\partial_{p}^{2} h(x, p)=0
$$

Proposition 10 The representation of the conformal group on the "undressed" fields (98)

$$
\varphi_{s}=\left.\left[U_{s+\frac{d-3}{2}}\left(\frac{i \hbar}{2} \vec{\rho}\right) h\right]\right|_{p^{s}}, \partial_{p}^{2} \varphi_{s}=0, \vec{\rho}=-i \partial_{x} \partial_{p}
$$

is diagonal w.r.t. s and has the form (370) with

$$
M_{a b}=-\left(p_{a} \delta_{b}-p_{b} \delta_{a}\right), \quad \gamma=2-s
$$

Proof. For Poincaré generators $P_{a}, J_{a b}$ and dilations $D$ the proof is trivial as for these generators there is no quantum correction because they commute with the undressing operator. The nontrivial part of the proof is to check that the quantum correction cancels 
for the special conformal transformations $K_{a}$. By direct calculation one proves that, for any function of $z=\frac{i \hbar}{2} \vec{\rho}$,

$$
\left[f(z), K_{a}\right]=-i \hbar^{2}\left(2\left(p \partial_{p}+\frac{d}{2}-1\right) f^{\prime}(z)+z f^{\prime \prime}(z)\right) \delta_{a}
$$

where the last equation is understood as valid when l.h.s. and r.h.s. act on traceless functions (i.e. those with $\partial_{p}^{2}=0$ ). Then, for the transformation laws of the undressed field $\varphi_{s}$ one has

$$
\begin{aligned}
& \delta_{K_{a}} \varphi_{s}=\left.\left[U_{s+\frac{d-3}{2}}\left(\frac{i \hbar}{2} \vec{\rho}\right) \delta_{K_{a}} h\right]\right|_{p^{s}}= \\
& \tilde{K}_{a} \varphi_{s}-\left.i \hbar^{2}\left\{\left(z U_{s+\frac{d-3}{2}}(z)+2\left(p \partial_{p}+\frac{d}{2}-1\right) U_{s+\frac{d-3}{2}}^{\prime}(z)+z U_{s+\frac{d-3}{2}}^{\prime \prime}(z)\right) \delta_{a} h\right\}\right|_{p^{s}}= \\
& \left.\tilde{K}_{a} \varphi_{s}-\left.i \hbar^{2}\left\{2\left(s+\frac{d}{2}-1\right)-(1+2 s+d-3)\right\}\left[U_{s+\frac{d-3}{2}}^{\prime}(z) \delta_{a} h\right\}\right|_{p^{s}}\right]= \\
& \tilde{K}_{a} \varphi_{s}, \gamma=2-s
\end{aligned}
$$

where in passing from the second to the third row we have used that $U_{\nu}(z)$ satisfies the equation (567).

Note that the value of the conformal dimension $\gamma=2-s$ is in accord with the structure of the free conformal spin-s action (329) which may be schematically written down as

$$
\mathcal{A}_{s}\left[\varphi_{s}\right] \sim \int d^{d} x \varphi_{s} \square^{\frac{d}{2}-2} \partial^{2 s} \varphi_{s}
$$

Needless to say, this value of conformal dimension in length units matches with that in mass units (61) derived from $A d S / C F T$ considerations.

As the gauge transformations (371) are linear, the global transformations are linear either, hence they present the symmetry of each term of the perturbative decomposition of the "quantized volume" around the vacuum $H=-p^{2}$. In particular, the quadratic action $\mathcal{A}_{2}$ is invariant up to boundary terms. As the transformations are diagonal w.r.t. spin, they present symmetry of each free conformal higher spin- $s$ theory with the action $\mathcal{A}_{s}\left[\varphi_{s}\right]$ (50),(53). Our derivation is a somewhat indirect, but the first, proof of the conformal invariance of the conformal higher spin theories.

\subsection{Algebra of observables of free quantized point particle and all bilinear conserved currents in general background fields.}

Here we simultaneously investigate global symmetries of the model and the Noether currents of the free action (133). 


\subsubsection{Algebra of observables}

Consider the action (133). The global symmetries are by definition the gauge transformations (137,138) which do not change the Hamiltonian. They act on the wave functions by the rule (138),

$$
\delta|\psi>=-\hat{\omega}| \psi>
$$

In accordance with (146) the infinitesimal global symmetries are characterized by the equation

$$
\omega^{\dagger} * H+H * \omega=0
$$

and hence, due to (134), leave the action (133) invariant up to boundary terms. The global symmetries form the Lie algebra $\mathcal{G}_{H}$ with commutation relations

$$
\begin{gathered}
{\left[\delta\left(\omega_{1}\right), \delta\left(\omega_{2}\right)\right]=\delta\left(\omega_{3}\right)} \\
\omega_{3}=-\left[\omega_{1}, \omega_{2}\right] .
\end{gathered}
$$

It is seen that the antihermitian global symmetries $\omega=i \epsilon$ commute with $H$ while hermitian ones $\omega=a$ anticommute with $H$.

The Lie algebra (383) possesses the subalgebra $\mathcal{G}_{\text {trivH }}$ of trivial symmetries

$$
\omega=i \mu * H \quad, \quad \mu=\mu^{\dagger}
$$

which annihilate every on-shell wave function, i.e. the one satisfying the equation (132). In fact the trivial symmetries form an ideal since given a trivial symmetry, its commutator with any symmetry is a trivial symmetry again:

$$
[i \mu * H, \omega]=i\left(-\mu * \omega^{\dagger}-\omega * \mu\right) * H
$$

As a consequence, the factor of the Lie algebra $\mathcal{G}_{H}$ by the ideal of trivial symmetries,

$$
\mathcal{G}_{o}=\mathcal{G}_{H} / \mathcal{G}_{\text {trivH }}
$$

is a Lie algebra which we call "algebra of observables".

The Lie algebra $\mathcal{G}_{H}$ admits a bilinear nonassociative multiplication

$$
\omega_{3} \equiv \omega_{1} \circ \omega_{2}=i\left(\omega_{1} * \omega_{2}+\omega_{2} * \omega_{1}\right)
$$

which is symmetric w.r.t. exchange of multipliers, and properly respects the trivial symmetries as the o-product of a trivial symmetry with any other one is a trivial symmetry again:

$$
(i \mu * H) \circ \omega=i\left(i \omega * \mu-i \mu * \omega^{\dagger}\right) * H
$$


Therefore, the algebra of observables $\mathcal{G}_{o}$ acquires o-product either: the o product of two elements from factor space $\mathcal{G}_{o}$ is given by the canonical projection of the o- product of their prototypes in $\mathcal{G}_{H}$. Analogously, any symmetrized product of symmetries gives a new symmetry:

$$
\begin{gathered}
\omega_{N} \equiv\left\{\omega_{1}, \ldots, \omega_{n}\right\}_{\circ} \equiv i^{N-1} \sum_{\text {permutations }\{1 \ldots N\}}\left(\omega_{1} * \ldots * \omega_{N}\right)= \\
2^{-N} \sum_{\text {permutations }\{1 \ldots N\}}\left(\omega_{1} \circ\left(\omega_{2} \circ \ldots \circ\left(\omega_{N-1} \circ \omega_{N}\right) \ldots\right)\right)
\end{gathered}
$$

This product which we call multi-o product, also respects properly the trivial symmetries, as $\omega_{N}$ is trivial if any of the multipliers is trivial.

\subsubsection{Noether currents}

Any global symmetry $\omega$ leave the action (133) invariant up to boundary terms, therefore there exists a Noether current $J_{\omega}$ given by bilinear expressions in $\bar{\psi}(x), \psi(x)$ and its derivatives. Let us recall a simple derivation of Noether theorem via studying general variation of an action w.r.t. transformation of the global symmetry but with a parameter being an arbitrary function of $x$. Let $S\left[\phi^{i}\right]$ be an action (depending on a set of fields $\left\{\phi^{i}\right\}$ ) invariant under the global symmetry $\delta \phi^{i}=\delta_{\nu} \phi^{i}$, where $\nu$ is a constant parameter.This means that the variation of the Lagrangian w.r.t. this transformation is a total derivative. Hence, if one makes the same transformation but with $\nu$ being an arbitrary function of $x$ the variation of the Lagrangian has a form

$$
\begin{aligned}
\delta L\left(\phi^{i}, \partial \phi^{i}, \partial^{2} \phi^{i}, \ldots\right) & =\nu_{, m} J^{m}\left(\phi^{i}, \partial \phi^{i}, \partial^{2} \phi^{i}, \ldots\right)+\text { total derivative }= \\
& -\nu \partial_{m} J^{m}+\text { total derivative. }
\end{aligned}
$$

Hence, if the fields $\phi^{i}$ satisfy classical equations of motion, the Noether current $J^{m}$ is conserved,

$$
\frac{\delta S}{\delta \phi_{i}}\left[\phi^{i}\right]=0 \Rightarrow \partial_{m} J^{m}\left(\phi^{i}, \partial \phi^{i}, \partial^{2} \phi^{i}, \ldots\right)=0 .
$$

The Noether currents are defined up to trivial ones of the form $J_{t r i v}^{m}=\partial_{n} k^{[m n]}$ with antisymmetric $k^{[m n]}$. The trivial symmetries are defined as

$$
\delta \phi^{i}=k^{[i j]} \frac{\partial S}{\partial \phi^{j}} ; k^{[i j]}=-k^{[j i]}
$$

and are easily seen to give rise to on-shell zero currents. Note that our definition of trivial symmetries (384) is a realization of a general one (392) applied to the action (133). 
Now we derive Noether currents associated with the global symmetries (382). To this end one has to study the variation of the action (133),

$$
\begin{aligned}
S[\psi] & =\frac{1}{2}<\psi|\hat{H}| \psi>+\frac{1}{2}(<\psi|\hat{H}| \psi>)^{*}= \\
& <\psi|\hat{H}| \psi>+ \text { boundary terms }
\end{aligned}
$$

w.r.t. transformations

$$
\delta|\psi>=-\nu(x) \hat{\omega}| \psi>
$$

where $\nu(x)$ is an arbitrary function of $x$.

The variation is

$$
\begin{gathered}
\delta S=-\frac{1}{2} \int d^{d} x\left\{(\nu \hat{\omega} \psi)^{*} \hat{H} \psi+\psi^{*} \hat{H} \nu \hat{\omega} \psi\right\}+c . c .= \\
=-\frac{1}{2} \int d^{d} x\left\{\psi^{*}\left(\hat{\omega}^{\dagger} \nu \hat{H} \psi+\hat{H} \nu \hat{\omega}\right) \psi\right\}+c . c .= \\
=-\frac{1}{2} \int d^{d} x\left\{\psi^{*}\left(\left[\hat{\omega}^{\dagger} \nu\right] \hat{H} \psi+[\hat{H} \nu] \hat{\omega}\right)+\nu\left(\hat{\omega}^{\dagger} \hat{H}+\hat{H} \hat{\omega}\right) \psi\right\}+c . c .
\end{gathered}
$$

where square brackets denote commutators. The first term may be neglected as it will give rise only to on-shell vanishing terms in $J^{m}$. The $\hat{\omega}^{\dagger} \hat{H}+\hat{H} \hat{\omega}$ term equals zero off-shell, due to (382). The remaining term in the variation is

$$
-\frac{1}{2} \int d^{d} x\left\{\psi^{*}[\hat{H} \nu] \hat{\omega} \psi\right\}+\text { c.c. }
$$

Using the representation (600) for an operator $\hat{H}$ with the Weyl symbol

$$
H(x, p)=\sum_{s=0}^{\infty} H^{m(s)}(x) p_{m_{1}} \ldots p_{m_{s}},
$$

one rewrites the last equation as

$$
\begin{gathered}
\delta S=-\frac{1}{2} \int d^{d} x \psi^{*} \sum_{s=0}^{\infty} \sum_{k=0}^{s} \sum_{l=1}^{s-k} \\
\left\{(-i \hbar)^{s}\left(\frac{1}{2}\right)^{k} C_{s}^{k} C_{s-k}^{l} H_{, m(k)}^{m(k) m(s-k)} \nu_{, m(l)}(\hat{\omega} \psi)_{, m(s-k-l)}\right\}+c . c .
\end{gathered}
$$

where $C_{s}^{k}=\frac{s !}{k !(s-k) !}$ are binomial coefficients. Integrating by parts, one represents the last expression in the form

$$
\delta S=\int d^{d} x \nu_{, m} J_{\omega}^{m}+\text { total derivative }
$$

where

$$
\begin{gathered}
J_{\omega}^{m}=-\frac{1}{2} \sum_{s=0}^{\infty} \sum_{k=0}^{s} \sum_{l=1}^{s-k} \\
\left\{(-i \hbar)^{s}\left(\frac{1}{2}\right)^{k}(-)^{l-1} C_{s}^{k} C_{s-k}^{l} \partial_{m_{1}} \ldots \partial_{m_{l-1}}\left[\psi^{*} H_{, m(k)}^{m(k) m(s-k)}(\hat{\omega} \psi)_{, m(s-k-l)}\right]\right\}+c . c .
\end{gathered}
$$


is a formula for the Noether current $J_{\omega}^{m}$ associated with the symmetry $\omega$ of the Hamiltonian $H$. It is also seen that the trivial symmetries of the form $\hat{\omega}=i \hat{\mu} \hat{H}$ give rise to the on-shell vanishing currents.

For the case of our main concern, the Hamiltonian of the form

$$
H=-p^{2}
$$

the expression for currents simplifies as only $s=2, k=0, l=1,2$ terms are nonzero. The result is

$$
J_{\omega}^{m}=-\frac{\hbar^{2}}{2}\left(\psi^{*} \partial^{m}(\hat{\omega} \psi)-\left(\partial^{m} \psi^{*}\right) \hat{\omega} \psi\right)+c . c .
$$

In order to get the complete list of conserved currents in this case one has to solve the global symmetry equation (382) in the case $H=-p^{2}$, which is rewritten as

$$
\left(p^{2}-\frac{\hbar^{2}}{4} \square\right) a(x, p)+p \partial_{x} \epsilon(x, p)=0
$$

and find the algebra of observables (386). We are interested only in global symmetries analytic in momenta (otherwise $\hat{\omega}$ is non-local). Using multi-o product one can construct the solutions of higher degree in momenta $p_{m}$ in terms of multi-o products of solutions of lower degrees in momenta. First of all, let us find the symmetries with $\epsilon$ linear in momenta and with $a$ independent of $p$,

$$
\epsilon=\xi^{m}(x) p_{m}, a=\alpha(x)
$$

Then (403) implies

$$
\begin{gathered}
\eta^{a b} \alpha=-\frac{1}{2}\left(\partial^{a} \xi^{b}+\partial^{b} \xi^{a}\right), \\
\square \alpha=0 .
\end{gathered}
$$

The first row is the definition of the conformal Killing vector $\xi^{m}$, with general solution of the form

$$
\begin{gathered}
\epsilon=\xi^{m}(x) p_{m}=b^{a} P_{a}+b^{a b} J_{a b}+c^{a} K_{a}+c D \\
P_{a}=p_{a}, J_{a b}=x_{a} p_{b}-x_{b} p_{a}, D=-(x, p), K_{a}=x^{2} p_{a}-2 x_{a}(x, p), \\
\alpha=-c+2 c^{a} x_{a},
\end{gathered}
$$

while the second row of (405) is satisfied as a consequence of the last row of (406).

The generators $P_{a}, J_{a b}, K_{a}, D$ form the conformal algebra $s o(d-q+1, q+1)$ of $d$ dimensional conformally flat space with $P_{a}, J_{a b}$ being Poincaré translations and Lorentz 
transformations and $K_{a}, D$ being the special conformal transformations and dilations, respectively. It is well-known the generators of the conformal algebra may be combined in the second-rank antisymmetric tensor $M_{A B}, A, B,=0 \ldots d-1, d, d+1$ (where $d$-th and $d+1$-th coordinates are space-like and time-like, respectively), according to the rule

$$
M_{a b}=J_{a b}, M_{+-}=-\frac{1}{2} D, M_{+a}=\frac{1}{2} P_{a}, M_{-a}=\frac{1}{2} K_{a},
$$

where $Y^{ \pm}=\frac{1}{2}\left(Y^{d} \pm Y^{d+1}\right)$.

Pure imaginary $\omega$ which belong to the center of the algebra of all phase-space functions,

$$
\omega=i \text { Const, } \text { Const } \in \mathbf{R}
$$

is a symmetry either (the localization of this symmetry is the conventional $U(1)$ gauge symmetry).

The multi-o products (389) of conformal generators

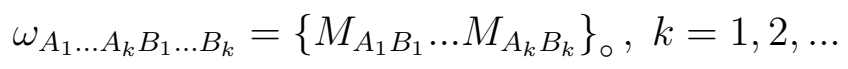

and $U(1)$-charge $\omega$ 's of the form (408) span general solution of the global symmetry equation (382,401,403), modulo trivial generators (384). The proof will be delivered elsewhere 54 [2]. Note that a multi-o product with $U(1)$ charge does not lead to new generators.

By construction, $\omega_{A_{1} \ldots A_{k} B_{1} \ldots B_{k}}$ are antisymmetric w.r.t. changes $A_{r} \leftrightarrow B_{r}$, and symmetric w.r.t. simultaneous change $A_{r} \leftrightarrow A_{r^{\prime}}, B_{r} \leftrightarrow B_{r^{\prime}}$. It is easy to prove that antisymmetrization of $\omega_{A_{1} \ldots A_{k} B_{1} \ldots B_{k}}$ w.r.t. any three indices equals zero. The most efficient way to see this is to use $d+2$-dimensional realization of the massless particle dynamics (see App. (G)), where the generators $M_{A B}$ are realized as $M_{A B}=Y_{A} \Pi_{B}-Y_{B} \Pi_{A}$ with $d+2$-dimensional coordinates $Y^{A}$ and momenta $\Pi_{B}$, then the antisymmetrization over three indices is zero because any third-rank antisymmetric tensor built from two vectors $Y_{A}, \Pi_{B}$, and $d+2$-metric $\eta_{A B}$, is zero. All this means $\omega_{A_{1} \ldots A_{k} B_{1} \ldots B_{k}}$ is characterized by the Young tableaux of the form

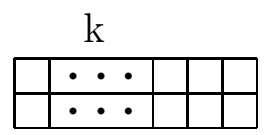

where the first row is occupied by $A$ 's and the second row by $B$ 's. In every $A B$ column one has the antisymmetry, that corresponds to the antisymmetry of $M_{A B}$ generators. At $k=0$, when the Young tableaux is empty, one has $\omega=i$ Const, Const $\in \mathbf{R}$.

\footnotetext{
${ }^{12} \mathrm{An}$ independent proof of this statement is in fact contained in paper 41, which has appeared when the present paper has been prepared for publication.
} 
The representation of the conformal group being a direct sum of irreducible ones characterized by traceless Young tableaux (410) with $k=0,1,2,3, \ldots$ is what we denote $\Upsilon_{d}$

If one applies symmetry generators (409) to the on-shell wave functions, i.e. those satisfying

$$
-p^{2} \psi(x)=\hbar^{2} \square \psi(x)=0,
$$

according to the rule (381), one can prove the following identity

$$
0=N_{A B} \psi(x)=\left\{\left(\hat{M}_{A B} \hat{M}_{C}^{B}+\hat{M}_{C B} \hat{M}_{A}^{B}\right)-\hbar^{2} \eta_{A C}(d-2)\right\} \psi(x),
$$

that is proved most simply in $d+2$-dimensional formalism (see Appendix (G)). As a consequence, the contraction of $\hat{\omega}_{A_{1} \ldots A_{k} B_{1} \ldots B_{k}} \psi(x)$ by any pair of $A, B$ indices reduces to the linear combination of $\omega_{A_{1} \ldots A_{k^{\prime}} B_{1} \ldots B_{k^{\prime}}} \psi(x), k^{\prime}<k$. (Indeed, after the contraction, one transfers the generators to be contracted to the right (by commuting them with the rest generators), to act on the wave function $\psi$, and then uses (412), all this results in multi-oproducts of $M_{A B}$ of a lower order). Thus, only traceless parts of $\hat{\omega}_{A_{1} \ldots A_{k} B_{1} \ldots B_{k}} \psi(x)$ are linearly independent. Studying $\omega$ 's acting on general on-shell wave function is equivalent to studying the observables (386) in the sense that the ideal of trivial symmetries is projected out in either cases. Therefore, the traceless parts of $\omega_{A_{1} \ldots A_{k} B_{1} \ldots B_{k}}$,

$$
\tilde{\omega}_{A_{1} \ldots A_{k} B_{1} \ldots B_{k}}=\text { traceless part of } \omega_{A_{1} \ldots A_{k} B_{1} \ldots B_{k}}
$$

are in one-to-one correspondence with the basis in the algebra of observables (380). In accordance with our considerations, one has the Noether currents (402)

$$
J_{A_{1} \ldots A_{k}, B_{1} \ldots B_{k}}^{m}
$$

associated with traceless symmetries (413). The on-shell currents automatically project out the trivial symmetries as trivial symmetries (384) give rise to the on-shell vanishing currents. Therefore, the space of on-shell conserved currents $(400$ is in one-to one correspondence with the algebra of observables. According to the above treatment, basis in the space of all on-shell conserved currents is provided by the currents (409), corresponding to the traceless Young tableaux (410), for $k=0,1,2, \ldots$ The $U(1)$-current corresponds to $k=0, \omega=i$ Const, Const $\in \mathbf{R}$. The energy-momentum and orbital momentum currents are $J_{a+}^{m}, J_{a b}^{m}$, respectively.

The traceless Young diagrams (410) are exactly those characterizing higher spin currents of free massless scalar constructed by Konstein, Vasiliev, and Zaikin by direct calculation [12]. Naturally, all the currents obtained in [12 are the linear combinations of our currents (402), modulo additions of "improvements" terms of the form 
$\delta J^{m}=\partial_{n} N^{m n}, N^{m n}=-N^{n m}$ which do not contribute to the conserved charges. Our derivation uncovers simple algebraic structure underlying the construction of currents, naturally explains the origin of two-row rectangular Young tableaux (410) and provides the simple unified formula (402) for all currents at once. Besides, the formula (400) provides an expression for all Noether currents of complex scalar field in arbitrary background, i.e. for an arbitrary wave operator $\hat{H}$.

\subsubsection{Classical global symmetries}

Let us recall that the classical covariance algebra $\mathcal{G}^{c l}$ consists of infinitesimal transformations which act on $H(x, p)$ by the rule

$$
\delta H(x, p)=2 a(x, p) H(x, p)+\{\epsilon, H(x, p)\} .
$$

Given Hamiltonian $H(x, p)$, the subalgebra of classical global symmetries $\mathcal{G}_{H}^{c l}$ is the subalgebra consisting of the elements $(\epsilon, a) \in \mathcal{G}^{c l}$ that preserve $H$,

$$
\delta_{\epsilon, a} H=2 a(x, p) H(x, p)+\{\epsilon, H(x, p)\}=0 .
$$

These equations are easily seen to be a contraction of the quantum ones (137), in accordance with discussion of Sec. (4.2). In fact, all the expressions below are obtained as a classical limit of corresponding quantum counterparts.

Given $\epsilon$, representing a global symmetry, its $a$-part is restored unambiguously from (416), so to specify the symmetry transformation it is enough to write down only its $\epsilon$-part, as we do in some places below. All global symmetries form an associative commutative algebra w.r.t product

$$
\left(\epsilon_{1}, a_{1}\right) \circ\left(\epsilon_{2}, a_{2}\right)=\left(\epsilon_{1} \epsilon_{2}, \epsilon_{1} a_{2}+\epsilon_{2} a_{1}\right)
$$

This associative algebra structure is compatible with the Lie algebra structure in the sense the commutator (124) is a derivation of the product $\circ$. It is easy to see that this product is nothing but a classical limit of the multi-o product (387), in fact, one obtains (417) from (387) if one neglects the order of product factors.

The trivial global symmetries are by definition those whose $\epsilon$-part vanishes on the constraint surface:

$$
\left(\epsilon_{t}, a_{t}\right)=\left(\tilde{\mu} H,-\frac{1}{2}\{\tilde{\mu}, H\}\right),
$$

where $\tilde{\mu}(x, p)$ is an arbitrary function, related to the analogous quantum parameter $\mu$

(384) as $\tilde{\mu}=\mu \hbar$. The trivial symmetries form the ideal $\mathcal{G}_{\text {trivH }}^{c l}$ in $\mathcal{G}_{H}^{c l}$ considered either as a Lie algebra w.r.t. commutator (124) or as an associative algebra w.r.t. o-product. The factor algebra

$$
\mathcal{G}_{o}^{c l}=\mathcal{G}_{H}^{c l} / \mathcal{G}_{\text {trivH }}^{c l}
$$


is called algebra of observables. Each element of $\mathcal{G}_{o}^{c l}$, except the central element $($ Const, 0$)$ is a nontrivial symmetry of the particle's classical action (112).

Let $H=-p^{2}$. Then the classical algebra of observables is a contraction of the conformal higher spin algebra, and is defined as follows. The generators are given by a pair $(\epsilon, a)$, where

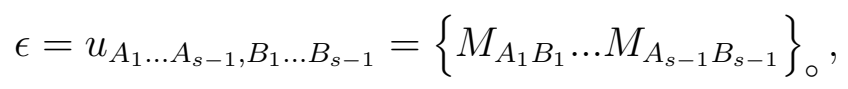

where $M_{A B}$ are generators of the conformal algebra (406), (407), while the multi-o product is classical (417). As in the quantum case, these generators are characterized by the Young tableaux (410). The factorization by the trivial generators (418) leads to the equation

$$
N_{A B}^{c l} \equiv M_{A B} M_{C}^{B}=0
$$

which holds on the constraint surface $p^{2}=0$. Just like in the quantum case, this equation ensures the tracelessness (after factorization by the ideal of trivial generators) of the generators 420 ) w.r.t. contraction of any two indices.

\subsubsection{Noether currents of the classical particle}

Let us show that all the currents (400) have their classical analogs derived by exactly the same procedure as the quantum currents. Given a classical global symmetry $\omega=(\epsilon, a)$, one derives, applying the Noether theorem, the currents $J^{\omega}[\psi]$ corresponding to each global symmetry $\hat{u}$. The answer is

$$
J_{\omega}^{m}(x)=\int d \tau \lambda \delta(x-x(\tau)) \epsilon(x(\tau), p(\tau)) \partial_{p_{m}} H(x(\tau) p(\tau))
$$

where $x(\tau), p(\tau), \lambda(t)$ is a solution for classical equations of motion. The currents are conserved as

$$
\begin{gathered}
\partial_{m} J_{\omega}^{m}=\int d \tau \lambda \epsilon \partial_{p_{m}} H \partial_{m} \delta(x-x(\tau))=-\int d \tau \lambda \epsilon\{H, \delta(x-x(\tau))\}= \\
=\int d \tau \epsilon \frac{d}{d \tau} \delta(x-x(\tau))=\int d \tau \frac{d}{d \tau} \epsilon \delta(x-x(\tau))=0
\end{gathered}
$$

as

$$
\frac{d}{d \tau} \epsilon(x(\tau), p(\tau))=\{\epsilon, H\}=-a H(x(\tau), p(\tau))=0
$$

\subsubsection{Noether currents of the Hamilton-Jacobi sigma-model}

Given a classical symmetry $\omega=(\epsilon, a)$, the variation of the action of Hamilton-Jacobi sigma model w.r.t. transformations with parameter $\nu(x) \omega$ reads

$$
\delta S_{c l}[\rho, \varphi]=\int d^{d} x \nu_{, m} J_{\omega}^{m}
$$


where

$$
J_{\omega}^{m}=\rho^{2}(x)\left(\epsilon \frac{\partial}{\partial_{p_{m}}} H\right)\left(x, \partial_{m} \varphi\right)
$$

are Noether currents. They are easily seen to conserve as a consequence of the equations of motions (158,159). One easily checks these currents are nothing but classical limit of the quantum currents (400), obtained by substituting (155) and keeping only first terms of the semiclassical expansion.

\section{Noether interaction of the scalar particle to gauge fields, and conformal higher spin fields}

Given the full set of conserved currents (400) it is natural to try constructing Noether interaction of the complex scalar field to the one-forms which take values in the algebra of observables, and try to formulate a sensible theory of these Yang-Mills fields. In this section we show that from the point of view of the influence on the particle dynamics the Noether interaction introduced in such a way is nothing but a very superfluous formulation of the interaction $\int d^{d} x \psi^{*} \hat{h} \psi$ and the only sensible part that is contained in gauge fields is that one contributing to $\hat{h}$.

\subsection{Quantum case}

Given the full set of (bilinear in $\psi$ ) conserved currents of the particle it is natural to construct first order interaction of the particle to some gauge fields by the rule

$$
S_{\text {int }}=e \sum_{\omega} \int d^{d} x J_{\omega}^{m}\left(\psi, \partial \psi, \ldots \psi^{*}, \partial \psi^{*}, \ldots\right) A_{m}^{\omega}(x)
$$

where $\psi$ is on-shell, i.e. $\hat{H} \psi=0, e$ is a coupling constant and the sum goes over the basis in the algebra of observables $\omega \in \mathcal{G}_{o}$, while $A_{m}^{\omega}(x)$ are gauge fields being subject to the abelian gauge transformations of the form

$$
\delta A_{m}^{\omega}(x)=\partial_{m} \epsilon^{\omega}(x)
$$

with gauge parameters $\epsilon^{\omega}(x)$. Needless to say, the action $S_{i n t}$ is gauge invariant w.r.t. these transformations. In fact, the gauge fields $A_{m}^{\omega}(x)$ are one-forms on $x$-space taking values in the linear space of the algebra of observables $\mathcal{G}_{o}$. Naturally, one constructs Yang Mills curvatures

$$
R_{m n}^{\omega}=\partial_{m} A_{n}^{\omega}-\partial_{n} A_{m}^{\omega}+e \sum_{\omega^{\prime}, \omega^{\prime \prime}} f_{\omega^{\prime}, \omega^{\prime \prime}}^{\omega} A_{m}^{\omega^{\prime}} A_{n}^{\omega^{\prime \prime}}
$$


where $f_{\omega^{\prime}, \omega^{\prime \prime}}^{\omega}$ are structure constants of $\mathcal{G}_{o}$, and tries to construct an action in terms of these curvatures for the newly introduced gauge fields $A_{m}^{\omega}(x)$. As the global symmetry of the free massless particle is the conformal higher spin algebra it is natural to anticipate one can construct a theory of conformal higher spin fields from $A_{m}^{\omega}(x)$. This kind of reasoning was employed by Fradkin and Linetsky in a series of papers where the authors used the same set of one-forms ${ }^{\mathrm{B}} \mathrm{a}$ and formulated the linearized dynamics in terms of $A_{m}^{\omega}(x)$ subject to some constraints and tried to generalize this to get interaction vertices. On of the reasons no progress had been achieved beyond the cubic order could be the excess in the number of gauge fields $A_{m}^{\omega}(x)$ as compared to the conformal higher spin fields described most naturally in terms of fluctuation of the hamiltonian $\hat{h}$.

To exhibit this excess let us demonstrate that the set of fields $A_{m}^{\omega}(x)$ is superfluous to gauge all the global symmetries of the scalar particle, and the Noether coupling is expressed in terms of special combinations of $A_{m}^{\omega}(x)$, which number equals the number of conformal higher spin fields. Indeed, let us use general formula (402) for Noether currents and integrate by parts to represent the expression (427) in the form

$$
\begin{gathered}
S_{\text {int }}=e \int d^{d} x \psi^{*}(x) \hat{h} \psi(x) \\
\hat{h}=-\frac{\hbar^{2}}{2} \sum_{\omega}\left\{2 A_{m}^{\omega}(x) \partial^{m}+\left(\partial^{m} A_{m}^{\omega}\right)\right\} \hat{\omega}+\text { hermitian conjugate }+\hat{\zeta}^{\dagger} \hat{p}^{2}+\hat{p}^{2} \hat{\zeta}
\end{gathered}
$$

The $\zeta, \zeta^{\dagger}$-terms account the ambiguity in the definition of $h$ arising because $\psi$ is on-shell, $\hat{p}^{2} \psi(x)=0$. It appears the Weyl symbol of operator $\hat{h}$ reads

$$
h(x, p)=-i \hbar \sum_{\omega}\left(\left(A_{m}^{\omega}(x) p^{m}\right) * \omega(x, p)-\omega^{\dagger}(x, p) *\left(A_{m}^{\omega}(x) p^{m}\right)\right)+\zeta^{\dagger} * p^{2}+p^{2} * \zeta,
$$

that may be checked by direct calculation using the formula (600) and its consequence $\widehat{A}^{m} p_{m}=-i \hbar\left(A^{m} \partial_{m}+\frac{1}{2}\left(A_{, m}^{m}\right)\right)$.

As a consequence of the abelian gauge invariance (428), $h(x, p)$ is subject to gauge transformations which may be easily deduced as follows

$$
\begin{gathered}
\delta A_{m}^{\omega} p^{m}=\partial_{m} \epsilon^{\omega} p^{m}=-i \hbar\left(p^{2} * \epsilon^{\omega}-\epsilon^{\omega} * p^{2}\right) \Rightarrow \\
\delta h(x, p)=\frac{2 i}{\hbar} \sum_{\omega}\left(\frac{i}{2 \hbar}\left(p^{2} * \epsilon^{\omega}-\epsilon^{\omega} * p^{2}\right) * \omega(x, p)-\omega^{\dagger}(x, p) * \frac{i}{2 \hbar}\left(p^{2} * \epsilon^{\omega}-\epsilon^{\omega} * p^{2}\right)\right)= \\
\left.=\frac{1}{2} \sum_{\omega}\left(p^{2} *\left[\epsilon^{\omega}, \omega\right]_{+}+\left[\epsilon^{\omega}, \omega^{\dagger}\right]_{+} * p^{2}\right)\right)= \\
=\tilde{\omega}_{\epsilon} *\left(-p^{2}\right)+\left(-p^{2}\right) * \tilde{\omega}_{\epsilon},
\end{gathered}
$$

\footnotetext{
${ }^{13}$ The coincidence of the algebra of global symmetries of the free massless particle and $d=4$ conformal higher spin algebras by Fradkin and Linetsky is the subject of the next section.
} 
where (in passing from the second to the third row) the definition of $\omega, \omega^{\dagger} p^{2}+p^{2} * \omega=0$, is used and

$$
\tilde{\omega}_{\epsilon}=-\frac{1}{2} \sum_{\omega}\left(\epsilon^{\omega} * \omega+\omega * \epsilon^{\omega}\right)
$$

But the set of fields $h(x, p)$ subject to gauge transformations (432) is nothing but the set of deformed conformal higher spin fields with $\mu^{2}=-\frac{\hbar^{2}}{4} \square$ i.e. the set of conformal higher spin fields. Therefore, one gets the following statement.

Proposition 11 General Noether coupling of the scalar particle to abelian gauge fields $A_{m}^{\omega}(x)$ is a superfluous formulation of the general coupling $S_{\text {int }}=e \int d^{d} x \psi^{*} \hat{h} \psi$ of the complex scalar field to conformal higher spin fields encoded in the operator $\hat{h}$ subject to gauge transformations $\delta \hat{h}=\hat{\tilde{\omega}} \hat{p}^{2}+\hat{p}^{2} \hat{\tilde{\omega}}$. The map from "superfluous" gauge fields $A_{m}^{\omega}$ and "superfluous" gauge parameters $\epsilon^{\omega}$ to the gauge fields $h(x, p)$ and gauge parameters $\tilde{\omega}$ is provided by eqs. (431) and (433).

\subsection{Classical and semiclassical case}

Using the currents (422) one constructs the Noether interaction of the classical particle to the abelian gauge fields $A_{m}^{\omega}$ as

$$
\begin{gathered}
S_{i n t}=e \sum_{\omega} \int d^{d} x J_{\omega}^{m}(x) A_{m}^{\omega}(x)= \\
=e \sum_{\omega} \int d^{d} x A_{m}^{\omega}(x) \int d \tau \lambda \delta(x-x(\tau)) \epsilon_{\omega}(x(\tau), p(\tau)) \partial_{p_{m}} H(x(\tau) p(\tau))= \\
=e \sum_{\omega} \int d \tau \lambda\left[\epsilon_{\omega} A_{m}^{\omega} \partial_{p_{m}} H\right](x(\tau), p(\tau)),
\end{gathered}
$$

where

$$
\omega=a_{\omega}+\frac{i}{\hbar} \epsilon_{\omega}
$$

As in the quantum case, this interaction is expressed via $h(x, p)$ as

$$
S_{\text {int }}=-e \int d \tau \lambda h(x(\tau), p(\tau))
$$

where

$$
h(x, p)=-\sum_{\omega} \epsilon_{\omega}(x, p) A_{m}^{\omega} \partial_{p_{m}} H+\zeta H,
$$

where $\zeta(x, p)$ is arbitrary. This is the classical version of the map (431). The gauge transformations $\delta A_{m}^{\omega}=\partial_{m} \epsilon^{\omega}$ are mapped to the conformal higher spin fields gauge transformations

$$
\delta h=\{\tilde{\epsilon}, H\}
$$


for $H=-p^{2}$ and

$$
\tilde{\epsilon}=-\sum_{\omega} \epsilon_{\omega}(x, p) \epsilon^{\omega}(x)
$$

which is nothing but the classical limit of the quantum relation (433).

If one introduces Noether interaction of the Hamilton-Jacobi sigma-model, one derives the same relations (437), (439) between the gauge transformations and gauge fields, while the interaction takes the form

$$
S_{i n t}=e \int d^{d} x \rho^{2} h(x, \partial \varphi)
$$

\subsection{On connection with Fradkin-Linetsky approach.}

As it's obvious from (431), the gauge field $A_{m}^{\omega}(x)$ enters all formulae only via combinations

$$
A^{\omega}(x, p)=A_{m}^{\omega}(x) p^{m}
$$

Consider the subset of $\omega$ 's of the form

$$
\omega_{a(s-1)}=\frac{i}{\hbar} p_{a_{1} \ldots} p_{a_{s-1}}, s=1,2, \ldots
$$

corresponding to all multi-o-products of Poincaré translations. Then the contribution of these $\omega$ 's to $h(x, p)$ is

$$
h(x, p)=\sum_{s=1}^{\infty}\left(A^{a(s-1) m}(x) p_{m}\right) * p_{a_{1}} \ldots p_{a_{s-1}}+c . c .=2 \sum_{s=1}^{\infty} A^{a(s-1) m} p_{m} p_{a_{1} \ldots} p_{a_{s-1}}+o\left(\hbar^{2}\right)
$$

Thus one has the relation

$$
h^{a(s)}(x)=2 A^{a(s-1) a}(x)+o\left(\hbar^{2}\right)
$$

where the right index $a$ in the superscript is the former one-form world index of $A_{m}^{a(s-1)}$, and complete symmetrization over all $a$ 's holds. The relation of this kind, expressing the true dynamical conformal higher spin fields via the linearized connections taking values in an infinite-dimensional "conformal higher spin algebra", are the basic input of "gauge description" of conformal higher spin fields, used by Fradkin and Linestky in $d=2,3,4$ [14] as a version of Vasiliev's "gauge description" [21] of Fronsdal higher spin gauge fields.

Contributions with another $\omega$ produce the analogous result. Other $\omega$ of the form (409) give rise to further contributions to $h(x, p)$ which however do not bring nothing new as (444) already provides $h(x, p)$ of general form. Thus, in the "gauge description" of conformal higher spin fields, one introduces a huge set of Yang-Mills fields which is much larger actual description of conformal higher spin fields calls for. 
As a consequence of this excess, in the "gauge description" one bumps with serious difficulties at the nonlinear level. In fact one has to imply some constraints to eliminate the superfluous fields at the nonlinear level, and these constraints are not known beyond the approximation of cubic interaction. It appears that due to certain mechanism these constraints are sufficient for constructing consistent cubic vertices [14], but advancement to next orders in interaction is prohibited without generalizing the constraints to further orders.

To summarize, our description in terms of the Hamiltonian $H(x, p)$, subject to gauge transformations (209) appears to be a more economic language for the description of conformal higher spin fields. On the other hands, taking in mind the full nonlinear picture provided in this paper it may be possible to find a proper nonlinear generalization of the Fradkin-Linetsky results, in particular, to find proper generalization of the constraints on auxiliary fields, and to express our "quantized volume" action (221) in terms of corresponding Yang-Mills curvatures.

A motivation for introduction of Yang-Mills fields was to gauge an infinite-dimensional global symmetry that was anticipated to arise in conformal higher spin models. It is clear from our consideration this is successfully achieved without introducing Yang-Mills fields. The remaining question is do our global symmetries (386) match with infinite-dimensional algebras Fradkin and Linetsky started from. The answer is yes. In fact, the conformal higher spin algebras, whenever constructed, exactly match the algebra of observables $\mathcal{G}_{o}$ of the massless particle. This match is the subject of the next section.

\section{On oscillator realizations of conformal higher spin algebras in $d=3,4,6$. Twistors.}

Here we will show the algebra of observables of the massless particle in flat $d$-dimensional Minkowski space $\operatorname{chs}(d-1,1)$ coincides with infinite-dimensional conformal higher spin algebras constructed previously by several authors.

As we have shown above w.r.t. its conformal subalgebra $s o(d, 2)$ this algebra is decomposed as a direct sum of finite-dimensional representations characterized by the infinite set of two-row rectangular Young tableaux (410) with spins $s=0,1,2,3, \ldots$, the latter representation is what we denote $\Upsilon_{d}$.

On the other hand the same algebra may play an important role in the theory of higher spin fields in $A d S_{d+1}$ as zero modes of linearized gauge transformations of the massless higher spin fields in $d+1$-dimensional AdS space are in one-to-one correspondence with generators of $\operatorname{chs}(d-1,1)$ [55]. Anticipating existence of consistent higher spin theory in 
$d+1$ dimensions, the zero modes of linearized gauge transformations are in one-to-one to global symmetries of the free action being a sum of Fronsdal theories of all integer spins from zero to infinity. Thus, $\operatorname{chs}(d-1,1)$ may be a global symmetry of free Fronsdal theories in $A d S_{d+1}$. This reasoning conforms with results by Lopatin and Vasiliev [16], who constructed free massless spin- $s$ theories corresponding to completely symmetric fields on $A d S_{d+1}$, in terms of one-forms and their linearized curvatures taking values in $\Upsilon_{d}$.

All this grounds the following definition of the higher spin algebras:

Definition 1 Higher spin algebra corresponding to totally symmetric higher spin fields in $D=d+1$ dimensional anti-de Sitter space is any algebra which contains so $(d, 2)$ as a subalgebra and decomposes w.r.t. it as $\Upsilon_{d}$ i.e. as direct sum of finite-dimensional representations, characterized by traceless two-row rectangular Young tableaux (410).

The uniqueness of the higher spin algebra is by no means guaranteed, families of solutions may exist.

As $s o(d, 2)$ is conformal algebra in $d$ dimensions, the same definition may be interpreted as the one for an infinite-dimensional symmetry algebra of conformal systems in $d$ dimensions (of course, we already know at least two of them: the first is free massless particle and the second is all-orders conformal higher spin theory of this paper), that justifies the definition

Definition 2 The conformal higher spin algebra in d-dimensional Minkowski space = higher spin algebra in $D=d+1$ dimensional anti- de Sitter space.

Till now, the boson higher spin algebras were known only for $d=3,4,6$, where they have emerged as an even part of the corresponding higher spin superalgebras via "oscillator" construction, the latter ascends to the famous family of isomorphisms $s o(2,1)=$ $s l(2, \mathbf{R}), s o(3,1)=s l(2, \mathbf{C}), s o(5,1)=s u^{*}(4)$ and $s o(3,2)=s p(4, R), s o(4,2)=s u(2,2)$, $s o(6,2)=s o^{*}(8)$ being neatly written in terms of division algebras $\mathbf{K}_{\nu}=\mathbf{R}, \mathbf{C}, \mathbf{H}, \nu=$ $1,2,4$ as $\operatorname{so}(\nu+1,1)=\operatorname{sl}\left(2, \mathbf{K}_{\nu}\right), \operatorname{so}(\nu+2,2)=\operatorname{sp}\left(4, \mathbf{K}_{\nu}\right)$ [36]. Let us show that in these particular cases our definition of the conformal higher spin algebras (386) reproduces the even subalgebras of the higher spin superalgebras in $d+1=4,5,7$ (equivalently, of the conformal higher spin superalgebras in $d=3,4,6)$ constructed previously by Vasiliev [21], Fradkin and Linetsky [14] and Sezgin and Sundell [32.

These superalgebras are constructed as Lie superalgebras corresponding to the enveloping algebras of a set of even oscillators

$$
\left[a^{I}, a^{J}\right]=0, \quad\left[b_{I}, b_{J}\right]=0, \quad\left[a^{I}, b_{J}\right]=\delta_{J}^{I}
$$


supplemented by some reality conditions, and factorized by certain ideals. The $s o(d, 2)$ is realized in terms of bilinear combinations of oscillators, while the oscillators transform w.r.t. $s o(d, 2)$ as spinors.

The point is that the origin of spinor generating elements is due to twistors representation of the massless particle dynamics in $d=3,4,6$ [34, [35]. Indeed, it is well-known that, in these dimensions, the mass-shell constraint $p^{2}=0$ may be solved via introducing even unconstrained spinors as

$$
p_{a}=\varsigma \bar{\lambda} \Gamma_{a} \lambda \Rightarrow p^{2} \equiv 0, \varsigma= \pm 1
$$

where $\Gamma_{a}$ are gamma-matrices, in $d=3 \lambda$ is a Majorana spinor, in $d=4 \lambda$ is a Weyl spinor, in $d=6 \lambda$ is Weyl or $s u(2)$ Majorana-Weyl spinor. $\varsigma$ accounts for the ambiguity in the choice of the connected component of the light cone, for one choice of $\varsigma$ one has $p_{0} \geq 0$, for another one $p_{0} \leq 0$.

After introduction of the second spinor

$$
\omega=i \varsigma x^{a} \Gamma_{a} \lambda
$$

the generators of the conformal algebra (406) appear to be rewritten in terms of bilinear combinations of $\lambda$ and $\omega$ in the manner

$$
\begin{gathered}
P_{a}=\varsigma \bar{\lambda} \Gamma_{a} \lambda, \quad K_{a} \sim \varsigma \bar{\omega} \Gamma_{a} \omega \\
M_{a b} \sim \bar{\lambda}\left[\Gamma_{a}, \Gamma_{b}\right] \omega+\text { c.c. }, \quad D \sim \bar{\lambda} \omega+\text { c.c. }
\end{gathered}
$$

Thus, any $s o(d, 2)$ generator is expressed either in terms of $\left(x^{a}, p_{b}\right)$ or in terms of $(\lambda, \omega)$. The commutation relations among any generators of $s o(d, 2)$ are reproduced if one considers $\lambda, \omega$ as a pair of canonically conjugated variables in a new, "twistor", phase space, like

$$
[\lambda, \lambda]=[\omega, \omega]=0,[l, \omega]=i \mathbf{1}
$$

According to the analysis of Sec. (10.2), an arbitrary element of the algebra of observables of the $d$-dimensional massless particle is represented by the equivalence class of polynomials built from the generators of conformal group $s o(d, 2)$, modulo the ideal of trivial observables in the enveloping algebra of $s o(d, 2)$ generated by the combination $N_{A B}(412)$

$$
g_{o} \in \mathcal{G}_{o}, \quad g_{o}=\sum_{k=0}^{\infty} K^{A_{1} \ldots A_{k} B_{1} \ldots B_{k}}\left\{M_{A_{1} B_{1}} \ldots M_{A_{k} B_{k}}\right\}_{\circ}
$$

\footnotetext{
${ }^{14}$ the pair $\lambda, \omega$ is said to form a "twistor".
} 


$$
\begin{gathered}
g_{o} \sim g_{o}+f_{1}^{A B} * N_{A B}+N_{A B} * f_{2}^{A B} \\
N_{A B}=\hat{M}_{A B} \hat{M}_{C}^{B}+\hat{M}_{C B} \hat{M}_{A}^{B}-\hbar^{2} \eta_{A C}(d-2),
\end{gathered}
$$

where $K^{A_{1} \ldots A_{k} B_{1} \ldots B_{k}}$ are real $s o(d, 2)$ tensors characterized by the traceless Young tableaux (410), and $f_{1,2}^{A B}$ are arbitrary elements of the form (450).

Any $g_{o}$ may be expressed in terms of the twistor $(\lambda, \omega)$, where the monomials of $n$-th degree in $M_{A B}$ turn into the ones of $2 n$-th degree in $(\lambda, \omega)$. We refer this representation of $\mathcal{G}_{o}$ as twistor picture. Note that, in the twistor picture, the elements of the ideal of trivial observables (451) are identically zero

$$
N_{A B}(\lambda, \omega) \equiv 0
$$

Therefore, each equivalence class (451) is unambiguously represented in the twistor picture, and one gets the homomorphism (448), (450) of the algebra of even power series in $(\lambda, \omega)$ to the algebra of observables of $d$-dimensional massless particle $\mathcal{G}_{o}$.

\subsection{Quaternion twistors. $D=7, d=6$, higher spin algebra $h s(8 *)$}

To show how it works we consider $d=6$ case, the $d=3,4$ ones are obtained then by the dimensional reduction, while $d=10$ case is more complicated and will be considered elsewhere [55]. We use the $s o(5,1)$ spinor formalism in the form given in [46], which we partially reproduce in Appendix (II) for the reader's convenience.

The mass-shell constraint $p^{2}=0$ is solved in terms of the $s u(2)$ Majorana-Weyl spinor

$$
\lambda^{\alpha k}, k=1,2, \quad \alpha=1,2,3,4,
$$

which obeys the reality constraint

$$
\left(\lambda^{\alpha k}\right)^{*}=I^{\dot{a}}{ }_{\beta} \varepsilon_{k l} \lambda^{\beta l}, \quad \varepsilon_{k l}=-\varepsilon_{l k}, \quad \varepsilon_{12}=1, I_{\beta}^{\dot{\alpha}} I_{\dot{\gamma}}^{* \beta}=-\delta_{\dot{\gamma}}^{\dot{\alpha}}
$$

as

$$
\begin{aligned}
& p_{a} \rightarrow p^{\alpha \beta}=p_{a}\left(\tilde{\sigma}^{a}\right)^{\alpha \beta}=\lambda_{k}^{\alpha} \lambda^{\beta k} \\
& p_{a}\left(\sigma^{a}\right)_{\alpha \beta}=p_{\alpha \beta}=-\frac{1}{2} \varepsilon_{\alpha \beta \gamma \delta} p^{\gamma \delta}
\end{aligned}
$$

where the notation like $A_{k} B^{k}=A_{k} B_{l} \varepsilon^{k l}$ is adopted. The equation $p^{2}=0$ is obeyed as

$$
p^{2}=-\frac{1}{4}(p \sigma)(p \tilde{\sigma})=-\frac{1}{4} p_{\alpha \beta} p^{\alpha \beta}=-\frac{1}{4}\left(-\frac{1}{2} \varepsilon_{\alpha \beta \chi \delta} p^{\chi \delta} p^{\alpha \beta}\right)==\frac{1}{8} \varepsilon_{\alpha \beta \gamma \delta} \lambda_{k}^{\alpha} \lambda^{\beta k} \lambda_{k^{\prime}}^{\gamma} \lambda^{\beta k^{\prime}}=0
$$


Introduce the second component of the twistor,

$$
\omega_{\alpha}^{k}=i(x \sigma)_{\alpha \beta} \lambda^{\beta k}
$$

which obeys the reality constraint

$$
\left(\omega_{\alpha}^{k}\right)^{*}=-I_{\dot{a}}^{\beta} \varepsilon_{k l} \omega_{\beta}^{l} .
$$

Then it is easy to rewrite the generator of special conformal transformations as follows

$$
\begin{aligned}
K_{a} \rightarrow K_{\alpha \beta}=K_{a}\left(\sigma^{a}\right)_{\alpha \beta} & =\left(x^{2} p_{a}-2 x_{a}(x p)\right)\left(\sigma^{a}\right)_{\alpha \beta}=(x \sigma)_{\alpha \gamma}(p \tilde{\sigma})^{\gamma \delta}(x \sigma)_{\delta \beta}= \\
= & (x \sigma)_{\alpha \gamma} \lambda_{k}^{\gamma} \lambda^{\delta k}(x \sigma)_{\delta \beta}=\omega_{\alpha k} \omega_{\beta}^{k} .
\end{aligned}
$$

The Lorentz generators are

$$
\begin{gathered}
M_{a b} \rightarrow M_{\alpha}{ }^{\beta}=M_{a b}\left(\sigma^{a b}\right)_{\alpha}{ }^{\beta}=-\frac{1}{4}((x \sigma)(p \tilde{\sigma})-(p \sigma)(x \tilde{\sigma}))_{\alpha}{ }^{\beta}= \\
=\frac{i}{2}\left(\omega_{\alpha k} \lambda^{\beta k}-\frac{1}{4} \delta_{\beta}^{\alpha} \omega_{\gamma k} \lambda^{\gamma k}\right),
\end{gathered}
$$

where it is used that

$$
(p \sigma)_{\alpha \beta}=p_{\alpha \beta}=-\frac{1}{2} \varepsilon_{\alpha \beta \gamma \delta} p^{\gamma \delta}, \quad(x \tilde{\sigma})^{\alpha \beta}=x^{\alpha \beta}=-\frac{1}{2} \varepsilon^{\alpha \beta \gamma \delta} x_{\gamma \delta} .
$$

The dilation generator is

$$
D=-\frac{1}{2}\left(x^{a} * p_{a}+p_{a} * x^{a}\right)=\frac{i}{8}\left(\lambda_{k}^{\alpha} \omega_{\alpha}^{k}+\omega_{\alpha}^{k} \lambda_{k}^{\alpha}\right) .
$$

Thus, any $s o(6,2)$ generator is expressed either in terms of $\left(x^{a}, p_{b}\right)$ or in terms of $\left(\lambda^{\alpha k}, \omega_{\beta}^{k^{\prime}}\right)$.

The commutation relations of $s o(d, 2)$ are satisfied if $\left(\lambda^{\alpha k}, \omega_{\beta}^{k^{\prime}}\right)$ are canonically conjugated variables with commutation relations

$$
\left[\lambda^{\alpha k}, \lambda^{\beta k^{\prime}}\right]=\left[\omega_{\alpha}^{k}, \omega_{\beta}^{k^{\prime}}\right]=0,\left[\lambda^{\alpha k}, \omega_{\beta}^{k^{\prime}}\right]=-2 i \delta_{\beta}^{\alpha} \varepsilon^{k k^{\prime}}
$$

The variables $\left(\lambda^{\alpha k}, \omega_{\beta}^{k^{\prime}}\right)$ form the quaternion twistor that transforms as a Dirac spinor w.r.t. conformal group so $(6,2)$.

The generators

$$
u_{k k^{\prime}}=\frac{1}{2}\left(\lambda_{k}^{\alpha} \omega_{\alpha k^{\prime}}+\lambda_{k^{\prime}}^{\alpha} \omega_{\alpha k}\right)
$$

are not expressed in terms of $(x, p)$, they form $s u(2)$ algebra that commutes to all $s o(d, 2)$ generators,

$$
\left[u_{k k^{\prime}}, M_{A B}\right]=0
$$

Vice versa, any function of $\left(\lambda_{k^{\prime}}^{\alpha}, \omega_{\alpha k}\right)$ that commutes to $u_{k k^{\prime}}$ is a polynomial in $M_{A B}$ (at the level of bilinear combinations of $\lambda, \omega$, this is clear as all $s u(2)$-invariants are just the 
contractions of $\left(\lambda_{k^{\prime}}^{\alpha}, \omega_{\alpha k}\right)$ w.r.t. $s u(2)$ indices, but these combinations are exactly $M_{A B}$ (455,459,460,461), quite analogously one proves this for any monomial of $\lambda, \omega$.

According to the analysis of Sec. (10.2), an arbitrary element of the algebra of observables of the 6-dimensional massless particle is represented by the equivalence class of polynomials built from the generators of conformal group so $(6,2)$, modulo the ideal of trivial observables in the enveloping algebra of $s o(6,2)$ generated by the combination (451)

$$
\begin{gathered}
g_{o} \in \mathcal{G}_{o}, \quad g_{o}=\sum_{k=0}^{\infty} K^{A_{1} \ldots A_{k} B_{1} \ldots B_{k}}\left\{M_{A_{1} B_{1}} \ldots M_{A_{k} B_{k}}\right\}_{\circ} \\
g_{o} \sim g_{o}+f_{1}^{A B} * N_{A B}+N_{A B} * f_{2}^{A B}
\end{gathered}
$$

where $K^{A_{1} \ldots A_{k} B_{1} \ldots B_{k}}$ are real $s o(d, 2)$ tensors characterized by the traceless Young tableaux (410), and $f_{1,2}^{A B}$ are arbitrary elements of the form (466).

Any $g_{o}$ may be expressed in terms of the twistor $\left(\lambda^{\alpha k}, \omega_{\beta}^{k^{\prime}}\right)$, where the monomials of $n$-th degree in $M_{A B}$ turn into the ones of $2 n$-th degree in $\left(\lambda^{\alpha k}, \omega_{\beta}^{k^{\prime}}\right)$. We refer this representation of $\mathcal{G}_{o}$ as twistor picture. In twistor picture, any $g_{o}$ is represented by an arbitrary power series built from even powers of $\left(\lambda^{\alpha k}, \omega_{\beta}^{k^{\prime}}\right)$, which commutes to $s u(2)$ generators (464)

$$
g_{o}=g\left(\lambda^{\alpha k}, \omega_{\beta}^{k^{\prime}}\right), \quad g\left(-\lambda^{\alpha k},-\omega_{\beta}^{k^{\prime}}\right)=g\left(\lambda^{\alpha k}, \omega_{\beta}^{k^{\prime}}\right), \quad\left[u_{k k^{\prime}}, g_{o}\right]=0
$$

Note that, in the twistor picture, the elements of the ideal of trivial observables (451) are identically zero

$$
N_{A B}(\lambda, \omega) \equiv 0
$$

that may be checked either by direct calculation or by noting that the last relation is nothing but the $s o(d, 2)$-covariant version of the mass-shell constraint $p^{2}=0$, the latter being the $N_{++}$-component of (469). Indeed, the function $p^{2}$ is not a scalar w.r.t. $s o(d, 2)$ generators, but a component of a finite-dimensional representation, the symmetric traceless tensor $N_{A B}$. Then, as the mass-shell constraint $p^{2}=0$ is satisfied by the very construction in the twistor picture, the rest components of $N_{A B}$ are zero either by virtue of the $s o(d, 2)$-invariance.

Thus, in twistor picture, the algebra of observables of the $6 d$ massless particle chs $(d-$ $1,1)$ is represented by arbitrary real (w.r.t. conjugation 454,458) ) even power series of the components of the twistor $\left(\lambda^{\alpha k}, \omega_{\beta}^{k^{\prime}}\right)$, which commutes to su(2) generators (464).

But this is nothing but the $h s(8 *)$ algebra of ref. [32]. Indeed, the authors of [32] constructed the $h s(8 *)$ in terms of $s o(6,1)$ Dirac spinors $y_{\hat{\alpha}}, \bar{y}_{\hat{\beta}}, \hat{\alpha}, \hat{\beta}=1 \ldots 8$, satisfying commutation relations

$$
\left[y_{\hat{\alpha}}, \bar{y}_{\hat{\beta}}\right]=2 C_{\hat{\alpha} \hat{\beta}}, \quad C_{\hat{\alpha} \hat{\beta}}=C_{\hat{\beta} \hat{\alpha}}
$$


These 8 pairs of oscillators are identified with 8 ones $\lambda^{\alpha k}, \omega_{\beta}^{k^{\prime}}$. The $s u(2)$ generators of 32 are built as 3 contractions

$$
\frac{i}{4} y_{\hat{\alpha}} y_{\hat{\beta}} C^{\hat{\alpha} \hat{\beta}}, \quad \frac{i}{4} \bar{y}_{\hat{\alpha}} \bar{y}_{\hat{\beta}} C^{\hat{\alpha} \hat{\beta}}, \quad \frac{1}{4} \bar{y}_{\hat{\beta}} y_{\hat{\alpha}} C^{\hat{\alpha} \hat{\beta}},
$$

which are identified with our $s u(2)$ generators (464). After these identifications, the construction of the $h s(8 *)$ coincides with our definition (468) up to a choice of a basis in the algebra. Thus one has the isomorphism

$$
\operatorname{chs}(5,1)=h s(8 *) \text {. }
$$

\subsection{Complex twistors. $D=5, d=4$, higher spin algebra as even subalgebra of $h s c^{0}(4)=h u_{0}(1,0 \mid 8)$.}

Here we use the Weyl spinor formalism adopted in [49]. As this formalism is well-known we do not dwell on details of notation. The mass shell-constraint $p^{2}=0$ is solved in terms of Weyl spinor $\lambda_{\alpha}, \alpha=1,2$

$$
p_{a} \rightarrow p_{\alpha \dot{\alpha}}=p_{a}\left(\sigma^{a}\right)_{\alpha \dot{\alpha}}=\lambda_{\alpha} \lambda_{\dot{\alpha}}, \quad \lambda_{\dot{\alpha}}=\left(\lambda_{\alpha}\right)^{\dagger}
$$

After introducing the second component of the twistor,

$$
\omega^{\alpha}=i x^{\alpha \dot{\alpha}} \lambda_{\dot{\alpha}}, \omega^{\dot{\alpha}}=-\left(\omega^{\alpha}\right)^{\dagger}
$$

the rest $s o(4,2)$ generators are rewritten as

$$
\begin{gathered}
K_{a} \rightarrow K_{\alpha \dot{\alpha}}=-\omega_{\alpha} \omega_{\dot{\alpha}} \\
M_{a b} \rightarrow M_{\alpha \beta}=\left(\sigma^{a b}\right)_{\alpha \beta} M_{a b}=\frac{i}{4}\left(\lambda_{\alpha} \omega_{\beta}+\omega_{\alpha} \lambda_{\beta}\right), D=-\frac{i}{2} \lambda^{\alpha} \omega_{\alpha}=\frac{i}{2} \lambda^{\dot{\alpha}} \omega_{\dot{\alpha}}
\end{gathered}
$$

The generators (475) form $s o(d, 2)$ either if they are realized in terms of $(x, p)$ or in terms of $\lambda, \omega, \bar{\lambda}, \bar{\omega}$ with commutation relations of the form

$$
\left[\lambda^{\alpha}, \omega_{\beta}\right]=-2 i \delta_{\beta}^{\alpha}, \quad\left[\lambda^{\dot{\alpha}}, \omega_{\dot{\beta}}\right]=2 i \delta_{\dot{\beta}}^{\dot{\alpha}},
$$

The spinors $\lambda_{\alpha}, \omega^{\beta}$ form the complex twistor which transforms as a Dirac spinor of the conformal group so $(4,2)$.

The analog of $s u(2)$ algebra of the previous subsection is the $u(1)$ algebra generated by

$$
u=i\left(\lambda^{\alpha} \omega_{\alpha}-\lambda^{\dot{\alpha}} \omega_{\dot{\alpha}}\right)-4,\left[u, M_{A B}\right]=0 .
$$

It easily seen that any function of $\lambda, \omega, \bar{\lambda}, \bar{\omega}$ that commutes to $u(1)$ is expressed via combinations of $M_{A B}$ and, in the contrast with the previous subsection case, $u(1)$. In the twistor 
picture, an arbitrary power series (450) is represented by $u(1)$-invariant even power series in $\left(\lambda^{\alpha}, \lambda^{\dot{\alpha}}, \omega^{\alpha}, \omega^{\dot{\alpha}}\right)$,

$$
g_{o}=g(\lambda, \omega, \bar{\lambda}, \bar{\omega}), \quad g(-\lambda,-\omega,-\bar{\lambda},-\bar{\omega})=g(\lambda, \omega, \bar{\lambda}, \bar{\omega}), \quad\left[u, g_{o}\right]=0
$$

Vice versa, if one considers arbitrary powers series of the form (478)

$$
g(\lambda, \omega, \bar{\lambda}, \bar{\omega}), \quad g(-\lambda,-\omega,-\bar{\lambda},-\bar{\omega})=g(\lambda, \omega, \bar{\lambda}, \bar{\omega}), \quad\left[u, g_{o}\right]=0
$$

then $\mathcal{G}_{o}$ arises as a Lie algebra constructed via commutators from the factor algebra of the associative algebra of power series (479) by the ideal generated by $u(1)$ :

$$
g \sim g+f u
$$

where $f$ is an arbitrary power series of the form (479). The equations (479,480) determine the Lie algebra $\mathcal{G}_{o}$ which is exactly the algebra $h s c^{0}(4)$ (where "hsc" ascends to "higher spin conformal") by Fradkin and Linetsky [14]. In fact, the authors of [14] constructed cubic interaction of the conformal higher spin fields in terms of $4 d$ Yang-Mills curvatures of the algebra $h s c^{\infty}$ which is nothing but the Lie algebra corresponding to the power series (479) without the factorization (480). Giving up the factorization results in the infinite multiplicity of each conformal spin in the spectrum. It was conjectured that any consistent all-orders interaction requires infinite multiplicity of spins [14. Our treatment shows this is not the case, as the model of this paper contains each spin with multiplicity one.

The same algebra $\mathcal{G}_{o}=h s c^{0}(4)$ is used recently in [23] in constructing, up to the cubic level, the action for higher spin massless fields corresponding to the totally symmetric Young tableaux, in $5 D$ anti de Sitter space. In [23] it is named $h u_{0}(1,0 \mid 8)$. The $s u(2,2)=s o(4,2)$ oscillators $\left(a_{\alpha}, b^{\beta}\right), \alpha, \beta=1,2,3,4$ in 23 are linear combinations of the components of the twistor $\left(\lambda^{\alpha}, \lambda^{\dot{\alpha}}, \omega^{\alpha}, \omega^{\dot{\alpha}}\right)$.

Thus one has the isomorphism

$$
\operatorname{chs}(3,1)=h s c^{0}(4)=h u_{0}(1,0 \mid 8)
$$

The algebra of observables $\mathcal{G}_{o}$ has a reduction to the power series, built from monomials of all odd degrees in generators $M_{A B}$. We denote this reduction $\mathcal{G}_{o}^{o d d}$. In the twistor picture, $\mathcal{G}_{o}^{o d d}$ is given in terms of powers series obeying the constraints

$$
g(-\lambda, \omega, \bar{\lambda},-\bar{\omega})=-g(\lambda, \omega, \bar{\lambda}, \bar{\omega}), \quad g(\lambda,-\omega,-\bar{\lambda}, \bar{\omega})=-g(\lambda, \omega, \bar{\lambda}, \bar{\omega})
$$

$\mathcal{G}_{o}^{\text {odd }}$ is isomorphic to $h o_{0}(1,0 \mid 8)$ of ref. 23] and to $h s(2,2)$ of [38 where it was used for the analysis of the linearized dynamics of the higher spin massless fields of even spins in $5 D$ anti-de Sitter space. Needless to say, the $s o(4,1)$ Dirac spinor $y^{\alpha}, a=1,2,3,4$ of [38] is related to the components of the twistor by linear combinations. 


\subsection{Real twistors. $D=4, d=3$, higher spin algebra as even subalgebra of the Vasiliev's higher spin superalgebra.}

This case is most easily obtained from the $D=5, d=4$ one by the reduction. One just has to impose the constraints

$$
\lambda_{\alpha}=\lambda_{\dot{\alpha}}, \omega^{\alpha}=\omega^{\dot{\alpha}}
$$

then for $s o(4,2)$ and $u(1)$ generators one gets

$$
u=0, p_{2}=0, K_{2}=0, M_{2 a}=0, a=0,1,3,
$$

and $s o(4,2)$ gets reduced to $s o(3,2)$. Thus, this case $\mathcal{G}_{o}$ is realized in terms of real oscillators $\left(\lambda^{\alpha}, \omega_{\beta}\right)$ forming the real twistor which transforms w.r.t. conformal group $\operatorname{so}(3,2)$ as a Dirac spinor. It is easy to see generators of $s o(3,2)$ are in one-to-one correspondence with all bilinear combinations of the components of the twistor.

W.r.t. $s o(3,1) \subset s o(3,2)$, the twistor transforms as $s l(2, \mathbf{C})$ Majorana spinor $y^{\alpha}, \bar{y}^{\dot{\alpha}}$, where $y$ 's are linear combinations of $\lambda^{\alpha}, \omega_{\beta}$, satisfying the commutation relations

$$
\left[y^{\alpha}, y^{\beta}\right]=2 i \epsilon^{\alpha \beta}, \quad\left[y^{\dot{\alpha}}, y^{\dot{\beta}}\right]=2 i \epsilon^{\dot{\alpha} \dot{\beta}}, \quad\left[y^{\alpha}, y^{\dot{\alpha}}\right]=0
$$

Therefore, $\mathcal{G}_{o}$ is isomorphic to the algebra od even power series built from oscillators (485). But this algebra is nothing but the even part of the superalgebra used by Vasiliev as a starting point for constructing cubic action for $4 D$ higher spin massless fields and for constructing full nonlinear equations of motion for this system in the "unfolded" form.

\section{Speculations}

Here we speculate on some issues concerning breaking the higher spin symmetries and then on the extension of the geometry "point particle + conformal higher spin fields in $d$ dimensions" to the one "tensionless $d-1$-brane + massless higher spin fields in $d+1$ dimensions".

\subsection{Higher spin compensator}

One may wonder why the strategy we have followed in the paper has led us to conformal higher spin theories which possesses such a huge infinite-dimensional gauge symmetry, instead of more conventional low spin Maxwell and Einstein gravity theories that possess only low spin gauge symmetries, namely, $U(1)$ transformations and $x$-diffeomorphisms. One of possible answers is that these theories may arise around the vacuum with a nonzero 
dilaton $D(x)$ since after the dilaton is gauge-fixed to a nonzero constant by Weyl dilations, the dilaton-curvature couplings $\int d^{d} x \sqrt{g} D^{\frac{d}{2}-1} R, \int d^{d} x \sqrt{g} D^{\frac{d}{2}-2} F_{m n} F^{m n}$ will provide the standard Einstein and Maxwell actions. However, we adhere a more radical point of view that the standard low spin physics arises when, besides Hamiltonian $H(x, p)$, a new "Higgs-like" field is added, which transforms w.r.t. canonical transformations and compensates all the higher spin gauge symmetries in the sense this "Higgs-like" field may be fully or partly eliminated by gauge transformations and after that the higher spin gauge symmetries get expired and the only remaining gauge transformations are $x$ diffeomorphisms and perhaps $U(1)$-Maxwell symmetries. With this new field added, new structures in the action become possible, and as a result after it is eliminated the new structures provide something like "mass terms" for conformal higher spin fields.

As conformal higher spin fields in $d$ dimensions arise as boundary values of massless higher spin fields on $d+1$-dimensional anti-de Sitter space [11], introduction of compensator to the theory of conformal higher spin fields can have the analog in the theory of massless higher spin fields in $A d S_{d+1}$. Thereby finding of the compensator may shed the light on the nature of "Higgsing" the theory of massless higher spin fields [9]. Below we briefly present a natural candidate on the role of this field, which we call "higher spin compensator". We suppose that the gauge group of the theory in the presence of a compensator remains the same. We describe only what could happen in a classical limit, i.e. when the gauge group is a semidirect product of canonical transformations and hyper Weyl ones. Moreover, for a moment we forget about hyper Weyl symmetry and discuss only the compensation of canonical transformations.

The higher spin compensator is provided by the prepotential of the symplectic form on the phase space. Consider the symplectic two-form

$$
\omega_{2}=2 d p_{m} \wedge d x^{m}
$$

It may be represented as a differential of a one-form $\omega_{1}$,

$$
\omega_{2}=d \omega_{1}
$$

where general solution for $\omega_{1}$ is

$$
\omega_{1}=2 p_{m} d x^{m}+d \beta(x, p),
$$

where $\beta(x, p)$ is an arbitrary power series in momenta, defined modulo transformations $\beta \sim \beta+$ Const. Under infinitesimal canonical transformations with generating function $\epsilon(x, p)$

$$
\delta x^{m}=\left\{x^{m}, \epsilon\right\}, \delta p_{m}=\left\{p_{m}, \epsilon\right\}
$$


the symplectic form is invariant. As a consequence, $\omega_{1}$ transform to another $\omega_{1}$ of the form (488), equivalently, $\beta$ transforms like

$$
\delta_{\epsilon} \beta=\{\epsilon, \beta\}+2\left(1-p \partial_{p}\right) \epsilon
$$

One checks by direct calculation this formula defines a realization of canonical transformations algebra, so that

$$
\left[\delta_{\epsilon_{1}}, \delta_{\epsilon_{2}}\right]=\delta_{\left\{\epsilon_{1}, \epsilon_{2}\right\}}
$$

Note the appearance of inhomogeneous term in (490) signalling of the Higgs-like nature of the field $\beta$.

Given one-form (486), (488), one constructs an outer automorphism $\mathcal{N}$ of the Lie algebra of canonical transformations, by the rule

$$
\mathcal{N} \epsilon=\frac{1}{2}\{\beta, \epsilon\}+\left(p \partial_{p}-1\right) \epsilon=-\frac{1}{2} \delta_{\epsilon} \beta
$$

One checks by direct calculation that this operation is an automorphism indeed, i.e.

$$
\mathcal{N}\left\{\epsilon_{1}, \epsilon_{2}\right\}=\left\{\mathcal{N} \epsilon_{1}, \epsilon_{2}\right\}+\left\{\epsilon_{1}, \mathcal{N} \epsilon_{2}\right\}
$$

As a consequence, if $\mathcal{N}$ is diagonalizable in a linear space of the phase space functions then the algebra of all canonical transformations may be graded, namely, let

$$
\mathcal{N} \epsilon_{k}=k \epsilon_{k}
$$

then

$$
\left\{\epsilon_{k}, \epsilon_{k^{\prime}}\right\}=\epsilon_{k+k^{\prime}}
$$

This implies the phase space functions which satisfy the equation

$$
\mathcal{N} \epsilon_{0}=0,
$$

form a subalgebra in the whole algebra of canonical transformations which we call low spin subalgebra corresponding to the one-form $\omega_{1}$. An equivalent definition of this subalgebra is $\epsilon_{0}$ are transformations that leave intact $\beta(x, p)=0$.

To explain the name "low spin subalgebra" let us consider the case

$$
\beta=0 \Rightarrow \mathcal{N}=p \partial_{p}-1
$$

The decomposition into direct sum of graded subspaces (494) corresponds to the expansion in momenta $p_{m}$, where the homogeneous polynomials of $k$-th degree in momenta form the subspace with $\mathcal{N}=k-1$. With our choice of the class of phase space function as power series in momenta, the degree $k$ is bounded from below and runs from -1 to infinity, that 
corresponds to the subspaces of homogeneous polynomials in momenta of order $k+1$. Then the subalgebra of $\epsilon_{0}$ is nothing but the subalgebra of $x$-diffeomorphisms $\epsilon_{0}=\xi^{m}(x) p_{m}$.

Thus, if one extends set of dynamical variables by adding $\beta(x, p)$ which transforms by the law (490), and then uses these gauge transformations to gauge $\beta$ to zero, then the remaining gauge transformations are just $x$-diffeomorphisms. As a result, the only remaining gauge field is a metric $g_{m n}(x)$, while all the higher spin fields would acquire mass terms. These mass terms arise from additional gauge-invariant structures built already not from $H(x, p)$ alone but from $H(x, p)$ together with $\beta(x, p)$. These structures originate from the fact that, in the presence of $\beta$, given a scalar $G(x, p)$, which transforms as

$$
\delta G(x, p)=\{\epsilon, G\}
$$

there exists an additional scalar of the form

$$
\Phi(x, p)=\mathcal{N} G=\frac{1}{2}\{\beta, G\}+\left(p \partial_{p}-1\right) G
$$

Therefore, from $H$ and $\beta$ one can construct many scalars like

$$
\begin{gathered}
\Phi_{k}(x, p)=\mathcal{N}^{k} H \\
\Phi_{k_{1} \ldots k_{r}}=\left\{\left\{\Phi_{k_{1}}, \Phi_{k_{2}}\right\} \ldots \Phi_{k_{r}}\right\}
\end{gathered}
$$

Thus one has the possibility of constructing a variety of canonically-invariant functionals like

$$
\mathcal{A}[H, \beta]=\int d^{d} x d^{d} p F\left(H, \Phi_{k_{1} \ldots k_{r}}\right),
$$

where $F$ is an arbitrary function of many variables. If $\beta=0$, the family of new scalars is reduced to combinations like

$$
\Phi_{k_{1} \ldots k_{r}}=\left\{\left\{\left(p \partial_{p}\right)^{k_{1}} H,\left(p \partial_{p}\right)^{k_{2}} H\right\} \ldots\left(p \partial_{p}\right)^{k_{r}} H\right\}
$$

and further structures, obtained by Poisson brackets of these combinations and by applying operator $p \partial_{p}-1$. Note that the canonically-invariant equation

$$
\Phi_{1}=(s-1) H \Leftrightarrow \mathcal{N} H=(s-1) H
$$

provides the spin-s truncation of the whole theory as for $\beta=0$ its solution consists of the homogeneous polynomials in momenta of order $s$. It is tempting to reformulate ordinary Einstein gravity on this language as it will provide description of gravity as of a spontaneously broken phase of a theory with large gauge group and therefore it may uncover some hidden symmetries of Einstein theory. 
The formulae we have delivered root in the contact geometry in $2 d-1$ dimensions [50, 47] which provide them with a clear geometric interpretation, but we do not dwell on details here. Here we just stress that the higher spin compensator $\beta$ (or, what is the same, prepotential $\omega_{1}$ of the symplectic form $\omega_{2}$ ) is not a field a single point particle is able to test. Indeed, a single point particle can test only conformal higher spin fields as we have demonstrated in this paper. In fact $\beta$ presents an additional structure that can be tested only by an ensemble of point particles with different masses. It appears when one studies interaction of point particles with different masses it is obligatory to introduce the higher spin compensator $\beta$ and thus one recovers the standard interaction via low spin massless gauge fields - gravitons and photons being made up from fluctuation of $H$ and $\beta$, while the interaction via conformal gauge fields being made up just from fluctuations of $H$ arises when $\beta$ decouples [55].

\subsection{From conformal higher spin fields to Fronsdal higher spin fields. Higher spin fields and tensionless $D-2$-brane.}

After the spontaneous breakdown of gauge symmetry which possibility is displayed in the previous section, the only remaining gauge symmetries are $x$-reparametrizations and thereby the only surviving massless field is the spin- 2 graviton described by the fluctuation of the metric $g_{m n}$. In this sense, this spontaneous breakdown may be characterized as an "ultimate" one.

At the same time, after a more delicate breakdown, more massless gauge fields may occur. In particular, one expects existence of such a compensator that there will arise Fronsdal massless higher spin gauge theories of spins $s=0,1,2,3, \ldots \infty$.

At the linearized level, the structure of this compensator is clear from the picture displayed in the beginning of this paper in Sec. (2.2.2). Namely, in addition to the set of symmetric traceless tensors $\varphi^{a(s)}(x)$ of ranks $s=0,1,2,3, \ldots$, representing the conformal higher spin fields and associated with the fluctuations of the Hamiltonian,

one has to introduce another set of symmetric traceless tensor fields $\chi^{a(s)}$ with gauge transformations (55). This set of fields represents fluctuation of some geometric structure yet to be uncovered [55]. We call this geometric structure hyper Weyl compensator.

We expect the unified theory that is formulated in terms of the Hamiltonian and hyper Weyl compensator may be found along the lines we have constructed the conformal higher spin theory. Namely, one has to find an adequate geometry with a source $O$, in the sense of definitions given in Introduction, where background fields are represented in terms of Hamiltonian and hyper Weyl compensator, and the covariance algebra is the same as in 
this paper. In the case hyper Weyl compensator decouples one recovers the theory of Hamiltonian alone and therefore, conformal higher spin theory. In the same case, the object $O$ should get reduced to the point particle.

Our preliminary results indicate that, in $D$ dimensions, $O$ is a tensionless $D-2$ brane. This conjecture is based on the interpretation of conformal higher spin fields as of boundary values of massless higher spin fields in $A d S_{D}$ as it is described in Sec. (2.2.3). Namely, in the $A d S / C F T$ correspondence, a free $A d S_{D}$ massless field $\Phi_{s}$ of spin $s$, corresponding to totally symmetric Young diagram is solved, in the framework of the Dirichlet-type problem, in terms of conformal higher spin fields $\varphi_{s}$ on the boundary of $A d S_{D}$. Then, by the very results of this paper, the full set of $\varphi_{s}$ is nothing but a general background for $d$-dimensional quantum particle with the wave function $\psi(x)$, and there exists natural first-order interaction in $d$ dimensions

$$
S_{\text {int }}=e \int d^{d} y \psi^{*}(y) \hat{h} \psi(y), \square \psi(y)=0,
$$

where

$$
\hat{h} \psi(y)=\sum_{s=0}^{\infty}(-i \hbar)^{s} \varphi^{a_{1} \ldots a_{s}}(y) \partial_{a_{1}} \ldots \partial_{a_{s}} \psi(y)+o\left(\hbar \partial_{b} \varphi^{a(s)}, \hbar^{2} \partial_{b} \partial_{c} \varphi^{a(s)}, \ldots\right), a=0,1, \ldots, d-1
$$

and $\varphi^{a_{1} \ldots a_{s}}(y)$ are just mentioned symmetric traceless tensor fields. As far as $\varphi^{a_{1} \ldots a_{s}}(y)$ are boundary values of $A d S_{d}$ higher spin massless fields $\Phi_{s}$, this implies the full set of $\Phi_{s}$ interacts with a $d$-dimensional theory which, being expanded around some vacuum, exhibits itself as a free $d$-dimensional quantized particle, or, equivalently, as a free complex scalar field in $d$ dimensions.

It appears that certain limit of this world volume theory is a particular case of a tensionless $D-2=d-1$-brane in $D$ dimensions. To show this is the case, first consider general action (133),

$$
S=\int d^{d} y \psi^{*}(x) \hat{H} \psi(x)
$$

and extract the leading term of the semiclassical expansion of this functional (see Sec. (4.3)), provided by the Hamilton-Jacobi sigma model (157),

$$
S_{i n t}\left[\psi=\rho e^{\frac{i}{\hbar} \varphi}\right]=e \int d^{d} y \sum_{s=0}^{\infty} \rho^{2} H^{a_{1} \ldots a_{s}}(y) \varphi_{a_{1}} \ldots \varphi_{a_{s}}+o(\hbar) .
$$

In the case $H^{a_{1} \ldots a_{s}}(y)=0, s \neq 2$ this action does describe tensionless $D-2=d$-1-brane in a $D$-dimensional gravity background, where $\varphi(y)$ describes the transverse motion of the $D$-1-dimensional world volume in $D$-dimensional space, while $\rho$ is a world-volume density, being somewhat analogous to the inverse Lagrange multiplier $\frac{1}{e(\tau)}$ in the massless particle action $S=\int d \tau \frac{\dot{x}^{2}}{2 e}$. 
Really, consider a tensionless $D-2$-brane in a gravity background in $D$-dimensional space (see e.g. [40]), with the world volume action

$$
S\left[V^{a}(y), x^{m}\left(y^{a}\right)\right]=\int d^{d} y V^{a}(y) V^{b}(y) x_{, a}^{m} x_{, b}^{n} g_{m n}(x(y)) .
$$

where $m=0 \ldots d, V^{a}$ is a vector density, that transforms w.r.t. $y$-diffeomorphisms as $\left(\operatorname{det}^{\frac{1}{2}}\left(\frac{\partial y^{\prime}}{\partial y}\right)\right) \frac{\partial y^{a}}{\partial y^{\prime b}} V^{\prime b}\left(y^{\prime}(y)\right)=V^{a}(y)$. Variation w.r.t. $V^{a}$ gives

$$
V^{b}(y) x_{, a}^{m} x_{, b}^{n} g_{m n}(x(y))=0,
$$

and therefore the metric induced on the brane is degenerate. Variation w.r.t. $x^{m}$ gives

$$
-2 \partial_{a}\left(V^{a} V^{b} g_{m n} X_{, b}^{n}\right)=0
$$

Using $D$-1-reparametrizations one can (locally on the world volume) impose the gauge

$$
x^{a}(y)=y^{a},
$$

which can be substituted back in action without spoiling the dynamics. Denoting

$$
x^{D-1}(y)=\varphi(y)
$$

one rewrites the equation (509) as

$$
V_{a}+\varphi_{, a}\left(V^{b} \varphi_{, b}\right) g_{D-1, D-1}\left(y^{a}, \varphi\right)=0
$$

where the indices are raised and lowered via $g_{a b}$ and its inverse (we have put for simplicity $\left.g_{a, d-1}=0\right)$. With the help of this equation $V^{a}$ is solved in terms of $\varphi$ up to a multiplier,

$$
V_{a}=\rho \varphi_{, a}
$$

where $\rho$ is a variable which could not be fixed from the equation (513). Another consequence of $(513)$ is the constraint

$$
g_{D-1, D-1}(y, \varphi) g^{a b}(y, \varphi) \varphi_{a} \varphi_{b}+1=0
$$

Among the remaining equations are those obtained by variation w.r.t. $x^{m}$. In the gauge (511) the only independent equation is that with $m=D-1$, which takes the form

$$
-\partial_{a}\left(\rho^{2} g^{a b} \varphi_{, b}\right)=0
$$

(here $g^{a b}$ is the inverse of $g_{a b}$ ). Thus, in the gauge (511), the dynamics of the tensionless $D-2$-brane is completely determined by two equations (516), (515). But if the metric 
does not depend on $(D-1)$-th coordinate these equations are nothing but the equations of motion of the action of Hamilton-Jacobi sigma model

$$
S[\rho, \varphi]=\int d^{d} y \rho^{2} H\left(y^{a}, \partial_{a} \varphi\right)
$$

with the Hamiltonian

$$
H\left(y^{a}, p_{a}\right)=-1-g_{D-1, D-1}(y) g^{a b}(y) p_{a} p_{b},
$$

which is a general quadratic Hamiltonian without linear and higher degrees in momenta (general nonzero dilaton may be obtained by re-scaling $\rho$.) Note also the essential difference of the Hamiltonian (518) with the Hamiltonian $H=-p^{2}$ which presents a vacuum of the $d$-dimensional conformal higher spin theory and governs dynamics of a massless $d$-dimensional particle. With flat metric and the nonzero dilaton $H(y, 0) \neq 0$, the Hamiltonian (518) is no longer a vacuum of the conformal higher spin theory.

To summarize, we have shown that a particular form of the D-1-dimensional HamiltonJacobi sigma model describes a tensionless D-2-brane in $D$ dimensions.

On the other hand, by the results of this paper, the quantum deformation of the Hamilton-Jacobi sigma model, being just the free action of the quantized particle (133), (157) exhibits interaction to conformal higher spin fields. At the same time, the latter fields may be viewed in the $A d S / C F T$ framework as boundary values of Fronsdal higher spin fields in $A d S_{D}$. Thus, massless higher spin fields in $A d S_{d}$ interact with a $d-1$ world volume theory, which in a certain limit describes a tensionless d-2-brane.

In this respect, the decoupling limit, when the hyper Weyl compensator decouples from the theory on the worldvolume, may have a transparent interpretation of the limit when the tensionless $d-2$-brane contracts to the tensionless 0-brane, i.e. to a massless particle.

We are led to conjecture that

Fronsdal higher spin fields in $D$ dimensions arise as background fields of an object $O$, which we call "tensionless D-2-brane". After neglecting certain higher derivatives terms in the worldvolume action, the dynamics of $O$ coincides with that of tensionless D-2-brane in D-dimensions. In another case, $O$ exhibits itself as a complex scalar field (quantized particle) in D-1 dimensions. There exists a contraction of $O$ 's dynamics when the worldvolume of $O$ collapses to a world line of a D-dimensional massless particle, after this contraction, one half of the off-shell Fronsdal gauge potentials (hyper Weyl compensator) decouples and one gets the system "point particle+conformal higher spin fields" described in this paper.

One can speculate there exists a limit when tensionless $D$-2-brane gets contracted not to a massless particle but to a tensionless string in $D$ dimensions. Then one gets natural 
coupling "tensionless string-higher spin fields". This conjecture is in accord with recent developments in $A d S / C F T$. Namely, in [8] and [9] it was conjectured that a particular limit of large- $N \mathcal{N}=4, d=4, S Y M$ theory, in which the t'Hooft coupling $g_{Y M}^{2} N$ tends to zero is dual to tensionless superstring in $A d S_{5} \times S^{5}$. The arguments the authors of these papers deliver are almost the same as ours, they are based, in fact, on the emergence of the infinite number of conserved currents in the free limit of SYM model. The essence of those arguments is that, in accordance with standard $A d S / C F T$ treatment, each current in $d=4$ couples to a gauge field, which is a boundary value of a massless field in $A d S_{5}$. At the same time, the fact that each massless field in $A d S_{D}$ has a dual conserved current built from complex massless scalar in $D-1$ [12] has got a simple proof in our treatment.

Let us make a few remarks. We see the phase of the wave function $\varphi$ is interpreted as the transverse position of the brane in $A d S_{d+1}$. This does not contradict to the periodicity of the phase as the period is easily seen to be $T=2 \pi \hbar$ and thus it is zero in the semiclassical limit we consider, so there is no condition like $\varphi=\varphi+T$ typical for a finite period. Next, the global $U(1)$-invariance $\psi^{\prime}=e^{\frac{i}{\hbar} \epsilon} \psi$ of the full quantum action (133) is translated to shift symmetry $\varphi \sim \varphi+\epsilon$ in terms of the phase, thus the action of the Hamilton-Jacobi sigma-model depends on $\varphi$ only via its derivatives, the latter fact conforms with independence of the background fields of $d$-1-brane on the transverse coordinate in $A d S_{D}$. Therefore, the construction of the full action of the brane in general background fields is related with breaking of $U(1)$ invariance either in the full quantum action $\int d^{d} x \psi^{*} \hat{H} \psi$ or in its classical limit, Hamilton- Jacobi sigma-model (157).

At the end of this subsection let us remark that the low-spin analogues of this section conjecture are well-known M2, D3, M5 branes living in backgrounds of M-theory vacua $A d S_{4} \times S^{7}, A d S_{5} \times S^{5}, A d S_{7} \times S^{4}$, the models which become, in the limit brane is disposed on the boundary of $A d S$, free conformal theories of real scalars describing transverse fluctuations of $D-2$ branes into $A d S_{D}$ and into corresponding spheres [27, [28]. These cases may be viewed as corresponding to broken "supergravity phase" of higher spin models, in this phase, the branes are massive and the higher spin gauge symmetries are broken so the only gauge fields are the ones of supergravity. In our case, the branes are tensionless and according to our conjecture there are massless fields of all spins in $A d S_{D}$. The real field describing fluctuations of tensionless brane inside $A d S_{D}$ is the phase $\varphi$ of the wave function $\psi=\rho e^{\frac{i}{\hbar} \varphi}$, it is not free because variation of the action (517) w.r.t. $\rho$ produces the constraint (515). The dynamics of $\varphi$ would match the dynamics of free real scalar if one puts the constraint

$$
\rho=\text { Const }
$$

inside the action (517), but then the action (517) ceases to be covariant w.r.t. all canonical 
transformations, as the last equation breaks canonical covariance. This is in accord with absence of higher spin symmetries in the "supergravity phase" (and in accord with Hull's results [13] on the absence of $W$-geometries in $d>2$ ( $\rho$ is absent in Hull's formulation $)$ ). We postpone more detailed analysis of this mechanism to future study [55].

\section{Conclusion}

Let us make a remark on non-scientific matters, mentioning that the main results of this paper has been anticipated by the author two years ago as it is manifested by the Eqs. (33) - (35) of Ref. [58 and the remark after Eq. (54) of that paper. However the structure of perturbative expansion remained rather obscure as off-shell one has fields of ever-increasing spin with infinite multiplicity of each symmetric traceless tensor. The progress followed after the paper [56] where it was realized the importance of "undressing" mechanism for rewriting quadratic action as a sum of standard conformal higher spin models.

To summarize, we have shown that the functional $\mathcal{A}[\hat{H}]$ which is equal to the trace of the projector to the positive eigenvalues subspace of an arbitrary Hermitian differential operator $\hat{H}$, is the action of conformal higher spin theory in the sense that, being expanded in perturbative series around the Euclidean flat vacuum $\hat{H}=\hbar^{2} \square$, $\mathcal{A}$ reproduces in quadratic approximation direct sum of free conformal higher spin gauge theories, the latter are higher-derivative models of order $\frac{d}{2}-2+2 s$ in derivatives formulated in terms of symmetric traceless tensors of all integer spins $s=0,1,2,3, \ldots$ The underlying gauge invariance has a very simple form of similarity transformation of $\hat{H}$ and in fact fixes the form of the action unambiguously. The latter invariance arises as covariance algebra of the quantized point particle (free complex scalar field) with $\hat{H}$ being its wave operator.

The covariance transformations preserving the vacuum are global symmetries of both the particle action and of the conformal higher spin theory action. Considered modulo so called trivial global symmetries (those proportional to Hamiltonian) the global symmetries define the algebra of observables which in the basic case $\hat{H}=\hbar^{2} \square$ are shown to form an infinite-dimensional algebra, the latter contains conformal algebra and decomposes w.r.t. its adjoint action as a direct sum of finite-dimensional irreducible representations characterized by all traceless two-row rectangular Young tableaux. The latter algebra probably arises as a global symmetry in the theory of higher spin massless fields of all integer spins in $A d S_{d+1}$.

Let us make a remark. When this paper has been in preparation, an interesting paper

41] has appeared, which studies global symmetries of the operator $\hat{H}=\hbar^{2} \square$ and uses 
almost the same construction of the infinite-dimensional symmetry algebra.

We have performed almost all calculations in the framework of well-known Weyl symbol formalism, which appears to be well-suited for our purposes. In particular, we have shown how to calculate the action in the form of a low-energy-like expansion in $\hbar$ counting number of space-time derivatives, in so doing, the zeroth, "classical" term of this expansion may be interpreted as a volume of the domain $H(x, p)>0$ in the phase space (where $H$ is a Weyl symbol of $\hat{H}$ ) while quantum corrections are represented by integrals of distributions localized on the constraint surface $H(x, p)=0$. It is $d-4+2 s$-th quantum correction to the classical term that provides in the quadratic approximation the conformal higher spin- $s$ action.

Note that although we have extensively used the star-product formalism we didn't introduce any kind of "unfolded formulation" [21] which in a sense replaces each field with all its derivatives at a fixed point of space-time. Most probably, if there exists a consistent reformulation of our model in "unfolded" form the star product of unfolded formulations will be the image of the star product in the particle phase space. This way the noncommutativity inherent to unfolded formulations of higher spin dynamics is induced by ordinary noncommutativity of quantum coordinates and momenta of a point particle. In particular, we have shown usual higher spin "star-product algebras" in $d=3,4,6$ constructed out of power series of even spinor oscillators are nothing but ordinary algebras of observables of the quantized particle, this way the spinor oscillators arise just as components of the twistor parameterizing phase space of the massless particle. Also in the framework of the paper there is no need in introducing unfolded formulations for realizing infinite-dimensional higher spin symmetries like it is done in [39], as in our case by construction one has the action of conformal higher spin algebra (382) on the wave function (381) and on fluctuations of gauge fields (374).

The model of this paper may be easily generalized to different cases of interest. Below we just mention the most immediate ones.

The description of conformal higher spin fields that carry inner indices of some finitedimensional group $\mathbf{G}$ is achieved by considering geometry with the source being Wong particle [45] which is nothing but an ordinary point particle supplemented by color degrees of freedom $Q^{i}, i=1 \ldots n$. The supersymmetrization of conformal higher spin theories may be achieved by considering geometry with the test source being Brink-Schwarz superparticle [42], note that as Brink-Schwarz superparticle is known to be well-defined only in backgrounds satisfying appropriate constraints [43], the extension to supersymmetric case has to provide higher spin generalization of those constraints. After that extension higher spin superalgebras should arise as superalgebras of observables of ( $N$-extended) 
massless Brink-Schwarz superparticle, with or without central charges. The origin of oscillator realizations of higher spin superalgebras, used by Vasiliev in the cases $d+1=4,5$ 23, 21] should become clear in this context as these dimensions $d=3,4$ are right those the so called doubly-supersymmetric formulation of superparticle dynamics becomes possible, which uses essentially the twistor representation of dynamics [37]. On the other hand, our definition does provide a wide class of superalgebras in any dimension, even when there is no a simple twistor representation of a massless vector in $d$ dimensions and therefore starting from any boson spinor oscillators (as it is conventional in Vasiliev's approach) is not possible.

Considering particles with higher time derivatives may lead to other interesting theories. In particular, there are backgrounds when higher derivative particles describe massless spinning particles with an arbitrary helicity [44, by analogy with the case of this paper one expects that these backgrounds could be vacua of some consistent gauge theories with infinite number of gauge fields.

One of the most intriguing developments of this paper would be constructing consistent theories of higher spin massless fields, which at the linearized level have twice as many fields as their conformal higher spin cousins provide. The additional fields form "hyper Weyl compensator" which does not influence dynamics of a single point particle. We speculate that a proper source that is able to test/emit massless higher spin fields in $d$ dimensions is an extended object that in some cases exhibits itself as a tensionless $d-2$-brane. In the limit the brane shrinks to a point the hyper Weyl compensator decouples and one recovers the geometry of the present paper "point particle+conformal higher spin fields". On the other hand, this reasoning conforms with recent conjecture on the "anti-holographic limit" of the $A d S / C F T$, where it was argued higher spin fields arise, in a tensionless limit, in the spectrum of IIB superstring on $A d S_{5} \times S^{5}$ with RRflux. Indeed, suppose our proposal about tensionless $d-2$-brane is correct, it implies natural coupling "tensionless $d-2$-brane/massless higher spin fields". Next, suppose in some situation tensionless brane collapses to a tensionless string, then there may survive coupling "tensionless string/massless higher spin fields". Due to basic stringy principles, massless higher spin fields should appear then in the spectrum of the firstly quantized tensionless string that is in accord with the "anti-holographic" conjecture [8], [9], [1].

Another way to draw a link to massless higher spin fields (which in fact motivated the conjecture of the previous paragraph) is to consider conformal higher spin fields on $d$-dimensional conformally flat space as boundary values of massless higher spin fields in $A d S_{d+1}$. Although low spin $(s=0,1,2)$ analogs of this phenomenon are in the core of $A d S / C F T$, the higher spin ones are less elaborated. Namely, the results on "anti- 
holographic" conjecture usually go along the way "free massless conformal matter in $d$-dimensions $\rightarrow$ infinite number of conserved currents in $d \rightarrow$ emitting of massless higher spin fields into the bulk of $A d S_{d+1}$ "[8], [9]. Perhaps results of our paper manifest it is worth extending this line by one more item to "free massless conformal matter in $d$ dimensions $\rightarrow$ infinite number of conserved currents in $d \rightarrow$ emitting of conformal higher spin fields into $d$-dimensional conformally flat space, with gauge-invariant action given by the "quantized volume" in the phase space of a particle $\rightarrow$ the latter fields arise as boundary values for Dirichlet-like problem for massless higher spin fields on $A d S_{d+1}$ ". The infinite number of conserved currents is provided, in our approach, by a free complex scalar field in $d$ dimensions, which in a certain (semiclassical) limit is shown to be interpreted as a tensionless $d-1$-brane in $d+1$ dimensions, where the phase of the complex scalar plays a role of the transverse position of the brane inside $d+1$-bulk, while the modulus of the field plays a role of a world volume density not related to the embedding of the brane into the bulk.

Note that our results are in accord with recent reasoning by Tseytlin [11] who argued that the action of free higher spin massless fields on $A d S_{5}$ evaluated on solutions of Dirichlet problem (with infra-red regulator $\epsilon$ restricting the limits of integration in $A d S_{5}$ ) should be equal to the quadratic approximation of the one-loop effective action $A=$ $-\frac{1}{2} \operatorname{Tr} \operatorname{Ln} \hat{H}$ of the operator $\hat{H}=-\square+\varphi_{s} \partial^{s} P_{s}$ in four dimensions, where $\varphi_{s}$ is a set of symmetric traceless tensors and $P_{s}$ are transverse traceless projectors related to the wave operators $L_{s}$ of spin-s conformal higher spin theories by the rule $L_{s}=\square^{s} P_{s}$. The quadratic term of the action $A$ is proportional to

$$
\sum_{s=0}^{\infty} \int d^{4} x \varphi_{s} L_{s} \ln \left(\epsilon^{2} \square\right) \varphi_{s}
$$

where $\epsilon$ is an ultra-violet regulator, and the logarithmically-divergent part of this expression is nothing but the sum of quadratic conformal higher spin theories. At the same time, the construction of the present paper provides all-order generalization of the sum of quadratic conformal higher spin actions. In our treatment, there are no tracelessness constraints on the fluctuations of $\hat{H}$, instead, one has hyper Weyl invariance that gauges away the traces at the linearized level and leads to proper decoupling phenomena at nonlinear level. As we argued in Sec. (6.2), in even dimension the full nonlinear action of conformal higher spin theory $\mathcal{A}[\hat{H}]$ is nothing but the logarithmically divergent term of the one-loop action $-\frac{1}{2} \operatorname{Tr} \operatorname{Ln} \hat{H}$, thus our statement matches with Tseytlin's result. On the other hand, recalling the AdS/CFT motivation of this treatment it is tempting to make the conjecture that in even dimensions $d>2$, the action of conformal higher spin theory is the logarithmically divergent term of the action of higher spin massless fields in 
$A d S_{d+1}$, evaluated on the solutions of Dirichlet-like problem As the action of higher spin massless fields is known, for $d>2$, only at cubic order in $A d S_{4}, A d S_{5}$ [3], [22], [23], the conjecture, if correct, provides important new data on all-orders interaction of higher spin massless fields.

Note that in even dimension our action equals (268), the time-independent term that appear in the asymptotic expansion of the trace of the evolution operator of $\hat{H}$, the latter term is a combination of Schwinger-Dewitt coefficients (267) typically encountered in calculation of Weyl anomaly, whence close connection of our treatment to Weyl anomaly considerations, either traditional [29] or "holographic" [30]. Indeed, the logarithmically divergent term of the $A d S$ action is known to produce "holographic" Weyl anomaly along the lines of refs [30. As in our model one has the higher spin extension of Weyl transformations to hyper Weyl ones it is interesting to extend the results of [30] to the higher spin case by using Weyl anomaly like considerations.

An important issue is the spontaneous breaking of higher spin gauge symmetries. We have presented a natural candidate on the role of corresponding Higgs-like field, "higher spin compensator" that appear to be a prepotential of the symplectic 2 -form in $d+d$ dimensional phase space. Considering coupling of this structure to conformal higher spin fields and to their "source", complex scalar field, one gets many new gauge-invariant structures as compared to the case higher spin compensator is absent. For complex scalar this would lead to the appearance of the self-interaction vertices and loss of conservation of infinite number of Noether currents (except $U(1)$ current and energy-momentum tensor) 91. For $A d S_{d+1}$ image of this picture one would get spontaneous breaking of higher spin gauge symmetries by some mass-like terms, rendering theory of higher spin massless fields into a model with low spin massless fields (scalar, photon, graviton) and an extensive collection of massive fields, the latter situation resembles string theory.

\section{Acknowledgements}

The author is indebted to A. Sharapov for fruitful collaboration on the initial stages of this project. The author is greatful to I. Bars, A. Barvinsky, R. Metsaev, D. Sorokin, B. Voronov, A. Tseytlin and I. Tyutin for useful discussions. The work is supported in part by grants RFBR No 02-02-16944, RFBR No 01-02-30024 and INTAS No 00-00262. 


\section{A On perturbative expansion in gauge theories}

Let $\mathcal{A}[H]$ be a functional of a set of fields $H$ invariant w.r.t. gauge transformations $\delta H=\delta_{g} H(H), g$ being a set of gauge parameters. Suppose $H=\bar{H}$ is a solution of the equations of motion,

$$
\frac{\delta \mathcal{A}[H]}{\delta H}[\bar{H}]=0 .
$$

The global symmetries are those gauge transformations preserving the vacuum $H=\bar{H}$. Consider the perturbative expansion of the action $\mathcal{A}[H]$ around this solution. Representing general background field $H$ as

$$
H=\bar{H}+h
$$

one expands the action in powers series in $h$ schematically like

$$
\begin{gathered}
\mathcal{A}[\bar{H}+h]=\mathcal{A}[\bar{H}]+\frac{1}{2 !} \frac{\partial^{2} \mathcal{A}}{\partial H \partial H}[\bar{H}] h h+\frac{1}{3 !} \frac{\partial^{3} \mathcal{A}}{\partial H \partial H \partial H}[\bar{H}] h h h+\ldots= \\
=\mathcal{A}_{0}+\mathcal{A}_{2}[h]+\mathcal{A}_{3}[h]+\ldots
\end{gathered}
$$

The $h$-linear term cancels as $\bar{H}$ is a solution of the equations of motion.

The following well-known propositions are used in the paper:

1. The quadratic action $\mathcal{A}_{2}[h]$ is gauge invariant w.r.t. linearized, $h$-independent gauge transformations given by the gauge variation of the "vacuum" $\bar{H}$,

$$
\delta_{g} h=\delta_{g} \bar{H}
$$

2. The equations of motions of the quadratic action are invariant w.r.t. all global symmetries. The global symmetries act on $h$ linearly, as the differential of the full transformation calculated at the vacuum point

$$
\delta_{\tilde{g}} h=\left(\frac{\partial \delta_{\tilde{g}} H}{\partial H}[\bar{H}]\right) h
$$

3. The cubic action is invariant w.r.t. linearized gauge transformations (523) if $h$ satisfies the linearized equations of motions.

$$
\frac{\partial^{2} \mathcal{A}}{\partial H \partial H}[\bar{H}] h=0 \Rightarrow \delta_{g} \mathcal{A}_{3}[h]=0
$$

\section{B Equations of motion.}

Here we undertake the analysis of the equations of motion of our theory in the formal operator approach. The variation of the action (197) is found easily as the order of product factors $H$ and $\delta H$ is inessential:

$$
\delta \mathcal{A}_{F}[H]=\operatorname{Tr}\left(F^{\prime}(H *) * \delta H\right)=\operatorname{Tr}\left(F^{\prime}(H *) \delta H\right)
$$


where it is used that for the Weyl *-product $\operatorname{Tr}(A * B)=\operatorname{Tr}(A B)$. Equations of motion take the form

$$
\frac{\delta \mathcal{A}_{F}[\bar{H}]}{\delta H}=F^{\prime}(H *)=0 .
$$

These equations are written in terms of a single function $F^{\prime}(H *)$ and encode the full nonlinear dynamics of the infinite collection of symmetric traceless tensor fields.

The equations, corresponding to variation of (197) w.r.t. particular space-time field $h^{m_{1} \ldots m_{i}}(x)$ are obtained from (527) as follows

$$
\begin{gathered}
\frac{\delta \mathcal{A}_{F}[H]}{\delta H^{m_{1} \ldots m_{i}(x)}}=\int d^{d} p \frac{\delta \mathcal{A}_{F}[H]}{\delta H} \frac{\delta H}{\delta H^{m_{1} \ldots m_{i}(x)}}= \\
\int d^{d} p \frac{\delta \mathcal{A}[H]}{\delta H} p_{m_{1} \ldots p_{m_{i}}}(x)=\int d^{d} p F^{\prime}(H *)(x, p) p_{m_{1} \ldots m_{i}}=0 .
\end{gathered}
$$

These are the equations which could have been obtained if one first evaluates the spacetime action by integrating over momenta in 197 and then varies it w.r.t. $H^{m_{1} \ldots m_{i}}(x)$. For general $F$ these equations are inevitably highly nonlinear and perhaps nonlocal, as general function $F^{\prime}(H *)$ has a form of an infinite semiclassical expansion (207) with $\hbar$ counting the total number of $x$-derivatives. Definitely, finding solutions to the equations written in this form is a hard task. Nevertheless, it appears that in the formal operator approach the equations of motion of the action (526) have a transparent interpretation.

The equation for symbols (527) may be rewritten as operator equation

$$
F^{\prime}(\hat{H})=0
$$

where $F$ is a function of operator $\hat{H}$. In terms of the full set of eigenvalues $H_{i}$ and eigenspaces $\mid H_{i}>$ for $\hat{H}$,

$$
\hat{H}\left|H_{i}>=H_{i}\right| H_{i}>,
$$

the equation (529) becomes equivalent to the condition

$$
F^{\prime}\left(H_{i}\right)=0 \quad \forall i
$$

Thus in the formal operator approach, an operator $\hat{H}$ is a solution of the classical equations of motion for the action $\mathcal{A}=\operatorname{Tr} F(\hat{H})$ if the spectrum of $\hat{H}$ is a subset of the set of zeroes of the function $F^{\prime}(\sigma)$. In other words, the function $F^{\prime}(\sigma)$ is an annulator of the Hamiltonian $\hat{H}$.

Therefore, if $F^{\prime}(\sigma)$ has only discrete set of zeros, then the spectrum $\left\{H_{i}\right\}$ should be discrete either. The operators $\hat{H}$ which have a nontrivial continuous spectrum can be a solution of equations of motion only if $F^{\prime}(\sigma)$ is zero in a continuous subset of $\mathbf{R}$.

Consider a simple example. Let $H$ be the Hamiltonian of $d$-dimensional Euclidean harmonic oscillator:

$$
H=\frac{1}{2}\left(\hat{p}^{2}+\alpha^{2} \hat{x}^{2}\right)-\hbar \alpha d / 2
$$


Note that $H \in \mathcal{C}$ in the sense of section (5.4). It is well known that it has the following discrete spectrum in $L^{2}\left(\mathbf{R}^{\mathbf{d}}\right)$ :

$$
H=\hbar \alpha n, n=0,1,2, \ldots
$$

It is easy to construct an annulator function. For example one may take $\left(\hbar^{\prime}=\alpha \hbar\right)$

$$
F^{\prime}(z)=\frac{e^{-\frac{2 C z^{\prime}}{\hbar}}}{\Gamma\left(-\frac{2 C z^{\prime}}{\hbar}\right)}=\prod_{k=1}^{\infty}\left(1-\frac{2 z}{\hbar^{\prime} k}\right) e^{\frac{2 z}{\hbar^{\prime} k}}
$$

where the infinite-product representation for $1 / \Gamma(z)$-function is used:

$$
\frac{1}{\Gamma(z)}=e^{-C z} z \prod_{k=1}^{\infty}\left(1+\frac{z}{k}\right) e^{-\frac{z}{k}}
$$

for $C$ being the Euler constant.

Therefore, one concludes that the Hamiltonian of harmonic oscillator corresponding to the background of flat Euclidean metric and certain $x$-dependent dilaton field is a solution of full nonlinear equations of motion determined by the function (534). Note that this simple example may be considered as a regularization of flat space Hamiltonian $(\alpha=0)$ which is not easy to work out along the lines of formal operator approach since it has the continuous spectrum.

Now let us take $F(\sigma)=\theta(\sigma)$. The equations of motion read

$$
\delta(\hat{H})=0
$$

$\delta(\sigma)$-function may be considered as a limit of an ordinary function which tends to zero everywhere except $\sigma=0$, and to infinity, when $\sigma=0$. Thus, the only sensible interpretation of the last equation is that $\hat{H}$ should not have zero eigenvalues in $L^{2}$. Note that the main case of this paper, the flat vacuum

$$
H=-p^{2}
$$

conforms with this interpretation, as this operator is known to have no normalizable zero modes. Thus we conjecture that a symbol $H(x, p)$ of an operator $\hat{H}$ is a solution of the equations of motion governed by the "quantized volume" action, if $\hat{H}$ has no normalizable zero modes in $L^{2}\left(\mathbf{R}^{d}\right)$. We stress however that as far as it concerns the actual dynamics of conformal higher spin fields, this interpretation, obtained in the formal operator approach, is fragile and should be used only as a motivation for more explicit study. 


\section{Gauge transformations for traceless tensors.}

Here we rewrite gauge transformations (69)

$$
\delta h(x, p)=-2 a(x, p)\left(p^{2}+\mu^{2}\right)-2 p_{m} \eta^{m n} \partial_{n} \epsilon(x, p)
$$

in terms of $a$-invariant traceless tensors.

We need some simple tools to handle traces of tensor coefficients of arbitrary functions. Let us note that, given any function

$$
F(x, p)=\sum_{k=0}^{\infty} F^{m(k)}(x) p_{m_{1}} \ldots p_{m_{k}}
$$

one can unambiguously represent it in the form

$$
F(x, p)=\sum_{l=0}^{\infty} \sum_{k=0}^{\infty} F_{(l)}^{m(k)}\left(p^{2}\right)^{l} p_{m_{1}} \ldots p_{m_{k}}
$$

where $F_{(l)}^{m(k)}$ are traceless, $F_{(l) n}{ }^{n m(k-2)}=0$. This is easily done by decomposing each $F^{m(k)}$ to its traceless part and the traces $F^{m(k)}=F_{(0)}^{m(k)}+\eta^{m(2)} F_{(1)}^{m(k-2)}+\eta^{m(2)} \eta^{m(2)} F_{(2)}^{m(k-4)}+\ldots$, then summing up the power series by momenta and noting that the trace parts give the powers of $p^{2}$. The decomposition (539) is then rewritten as

$$
F(x, p)=\sum_{k=0}^{\infty} F^{m(k)}\left(p^{2}\right) p_{m_{1}} \ldots p_{m_{k}}
$$

where $F^{m(k)}\left(p^{2}\right)=\sum_{l=0}^{\infty} F_{(l)}^{m(k)}\left(p^{2}\right)^{l}$. Decomposing the power series $F^{m(k)}(\sigma)$ at the point $\sigma=-\mu^{2}$ one gets

$$
\begin{gathered}
F(x, p)=\sum_{k=0}^{\infty} F_{\left[-\mu^{2}\right]}^{m(k)}\left(p^{2}+\mu^{2}\right) p_{m_{1}} \ldots p_{m_{k}}= \\
\sum_{l=0}^{\infty} \sum_{k=0}^{\infty}\left(p^{2}+\mu^{2}\right)^{l} F_{\left[-\mu^{2}\right](l)}^{m(k)} p_{m_{1}} \ldots p_{m_{k}}=\sum_{l=0}^{\infty}\left(p^{2}+\mu^{2}\right)^{l} F_{\left[-\mu^{2}\right](l)},
\end{gathered}
$$

where the power series $F_{\left[-\mu^{2}\right](l)}$ contain only traceless coefficients. Given $\mu^{2}$, we will say that the $F_{\left[-\mu^{2}\right](0)}$ term is the traceless part of the function $F(x, p)$ and the first, second and further traces of $F$ are represented by $\left(p^{2}+\mu^{2}\right)^{l} F_{\left[-\mu^{2}\right](l)}$ with $l=1,2, \ldots$ forming altogether the traceful part of $F$. The function is traceless if it is equal to its traceless part. In this sense each coefficient $F_{\left[-m^{2}\right](l)}$ is a traceless function.

Now represent all the entries of the gauge transformation laws (538) in the form (541) to get

$$
\delta \sum_{l=0}^{\infty}\left(p^{2}+\mu^{2}\right)^{l} h_{\left[-\mu^{2}\right](l)}=\sum_{l=0}^{\infty}\left\{-2\left(p^{2}+\mu^{2}\right)^{l+1} a_{\left[-\mu^{2}\right](l)}-2\left(p^{2}+\mu^{2}\right)^{l} p^{m} \partial_{m} \epsilon_{\left[-\mu^{2}\right](l)}\right\}
$$


wherefrom it is seen that all the traces of $h$ may be gauged away by $a$-transformations. In fact, the very destination of $a$ is to gauge away the traces of $h$. It is worth noting that the traceful part of $\epsilon$ is already contained in $a$ as the gauge transformations (542) do not change if one redefines $\epsilon, a$ according to

$$
\delta \epsilon=\left(p^{2}+\mu^{2}\right) \nu, \delta a=-p^{m} \partial_{m} \nu
$$

Therefore without loosing generality one may set $\epsilon$ traceless

$$
\epsilon=\epsilon_{\left[-\mu^{2}\right] 0} \equiv-2 \varepsilon=-2 \sum_{k=0}^{\infty} \varepsilon^{m(k)} p_{m_{1} \ldots} \ldots p_{m_{k}} ; \varepsilon_{n}{ }^{n m(k-2)}=0
$$

For any action $\mathcal{A}[h]$ invariant w.r.t. gauge transformations (538), $h$ should enter $\mathcal{A}[h]$ in $a$ - and $c$-invariant combinations only as far as $a$ transformations are purely algebraic. It is easy to see that the only $a$-invariant is the traceless function

$$
\varphi=h_{\left[-\mu^{2}\right](0)} .
$$

It is easy to derive the $\varepsilon$ transformation laws for the coefficients of $\varphi$ which read

$$
\delta \varphi^{m(s)}=\left(\text { Traceless part of } \partial^{m} \varepsilon^{m(s-1)}\right)-\mu^{2} \frac{s+1}{2 s+d} \partial_{n} \varepsilon^{n m(s)} .
$$

For $\mu^{2}=0$, these are the gauge transformations of conformal higher spin theories, which are seen to decay into independent subsystems described in terms of rank-s traceless tensor and rank- $(s-1)$ traceless parameter. For $\mu^{2} \neq 0$, as it is proved in the main text, for each spin $s$ there arises a deformation which appears to be related to its $\mu=0$ counterpart by undressing and reconstruction maps studied in Sec. (3.2), (3.3).

\section{Generating functions for integration over $d$-ball and $d-1$-sphere}

Given the Euclidean metric $g^{m n}=\delta^{m n}$ in a $d$-dimensional space parameterized by $p_{m}$, one defines the "constraint surface" as a $d-1$-sphere

$$
p^{2} \equiv g^{m n} p_{m} p_{n}=\mu^{2}, \quad \mu \in \mathbf{R}
$$

The integrals to be calculated are

$$
I_{m_{1} \ldots m_{s}}^{(k)}(x)=\int d^{d} p \theta^{(k)}\left(\mu^{2}-p^{2}\right) p_{m_{1} \ldots p_{m_{s}}}
$$


where $\theta^{(k)}$ is the $k$-th derivative of the "step" $\theta$-function $\theta(\sigma)$ :

$$
\begin{aligned}
& \theta(\sigma)=0, \quad \sigma<0 \\
& \theta(\sigma)=\frac{1}{2}, \quad \sigma=0 \\
& \theta(\sigma)=1, \quad \sigma>0 .
\end{aligned}
$$

In fact only even- $s$ integrals are to be calculated, because odd- $s$ integrals equal zero.

The first derivative of $\theta$-function is $\delta$-function, etc.,

$$
\partial_{\sigma}^{k} \theta(\sigma)=\delta^{(k-1)}(\sigma)
$$

Therefore, all the integrals (548) are well-defined: for $k=0$ the actual integration goes over the $d$-ball inside the constraint surface, while for $k>0$ the integrals are localized on the constraint surface being the $d-1$-sphere. The simplest case is

$$
I^{(0)}=\int d^{d} p \theta\left(\mu^{2}-p^{2}\right)=v_{d} \mu^{d}
$$

where

$$
v_{d}=\frac{\pi^{\frac{d}{2}}}{\Gamma\left(\frac{d}{2}+1\right)}
$$

is the volume of a unit ball in $d$ dimensions.

Due to (550), the integrals for different $k$ are related by differentiation by $\mu^{2}$ :

$$
I_{m_{1} \ldots m_{s}}^{(k)}=\left(\frac{\partial}{\partial \mu^{2}}\right)^{k} I_{m_{1} \ldots m_{s}}^{(0)} .
$$

The collection of all the integrals (548) is described by the generating functions

$$
G_{d}^{(k)}(l)=\sum_{s=0}^{\infty} \frac{i^{s}}{s !} I_{m_{1} \ldots m_{s}}^{(0)}(x) l^{m_{1}} \ldots l^{m_{s}}=\int d^{d} p \theta^{(k)}\left(\mu^{2}-p^{2}\right) e^{i p_{m} l^{m}}
$$

which depend on the auxiliary vector variable $l^{m}$. Likewise $I_{m_{1} \ldots m_{s}}^{(k)}$ the generating functions $G_{d}^{(k)}(l)$ are related by differentiation by $\mu^{2}$,

$$
G_{d}^{(k)}(l)=\left(\frac{\partial}{\partial \mu^{2}}\right)^{k} G_{d}^{(0)}(l)
$$

so all generating functions may be obtained from any one either by differentiation or by integration. It is convenient to calculate $G_{d}^{(1)}(l)$,

$$
G_{d}^{(1)}(l)=\int d^{d} p \delta\left(\mu^{2}-p^{2}\right) e^{i p_{m} l^{m}}
$$

which satisfies differential equation

$$
g^{m n} \frac{\partial}{\partial l^{n}} \frac{\partial}{\partial l^{m}} G_{d}^{(1)}(l)=-\mu^{2} G_{d}^{(1)}(l),
$$


as $\delta\left(\mu^{2}-p^{2}\right)\left(\mu^{2}-p^{2}\right)=0$. The integrals exhibit $s o(d)$ invariance. Hence the result of integration should depend only on $l^{2}=l^{n} l^{n}$, the generating function depends on $l^{m}$ only via single variable $l=\sqrt{l^{2}}$. Then the equation (557) reads

$$
l^{1-d} \partial_{l} l^{d-1} \partial_{l} G_{d}^{(1)}(l)=-\mu^{2} G_{d}^{(1)}(l)
$$

The substitution

$$
G_{d}^{(1)}(l)=l^{1-\frac{d}{2}} f(\mu l)
$$

turns this equation into the Bessel's equation,

$$
\left(\sigma^{2} \partial_{\sigma}^{2}+\sigma \partial_{\sigma}+\sigma^{2}-\left(\frac{d}{2}-1\right)^{2}\right) f(\sigma)=0 .
$$

The solution should be regular at $\sigma=0$ as

$$
G_{d}^{(1)}(0)=\left(\frac{\partial}{\partial \mu^{2}}\right) \int d^{d} p \theta\left(\mu^{2}-p^{2}\right)=v_{d} \frac{d}{2} \mu^{d-2} .
$$

Among the solutions with integer or half-integer indices, only the Bessel's function of the first kind are regular at the origin, therefore the correct solution for (560) is

$$
f(\sigma)=\kappa J_{\frac{d}{2}-1}(\sigma)
$$

where $\kappa$ is a $\mu$-dependent constant, $J_{\frac{d}{2}-1}(\sigma)$ is the Bessel's function of the first kind, of index $\frac{d}{2}-1$, see App. (因). Matching the behavior of $J_{\frac{d}{2}-1}(\sigma)$ at the origin with the "boundary condition" (561) one gets the final result

$$
G_{d}^{(1)}(l)=2^{\frac{d}{2}-1} \pi^{\frac{d}{2}} \mu^{\frac{d}{2}-1} l^{1-\frac{d}{2}} J_{\frac{d}{2}-1}(\mu l)
$$

This formula contains only even powers of $l$ as it should. $G_{d}^{(k)}$ 's can be easily obtained by making use of the identities (567).

$$
G_{d}^{(k)}(l)=2^{\frac{d}{2}-k} \pi^{\frac{d}{2}} \mu^{\frac{d}{2}-k} l^{-\frac{d}{2}+k} J_{\frac{d}{2}-k}(\mu l)
$$

Rewriting the generating functions as power series in $l^{m}$ provides all the integrals (548) as tensor powers of the metric $\delta^{m n}$ multiplied by certain $\mu$-monomials.

\section{E Bessel's functions}

Here we collect the properties of Bessel's functions of the first kind [52] used in the main text. Bessel's function of the complex variable $z \in \mathbf{C}$, of the first kind, of index $p \in \mathbf{C}$ is

$$
J_{\nu}(z)=\left(\frac{z}{2}\right)^{\nu} \sum_{k=0}^{\infty} \frac{(-)^{k}}{k ! \Gamma(\nu+1+\kappa)}\left(\frac{z}{2}\right)^{2 k} .
$$


The series (565) converges for any finite $z$ and $\nu$. It satisfies the differential equation

$$
\left(z^{2} \partial_{z}^{2}+z \partial_{z}+z^{2}-\nu^{2}\right) J_{\nu}(z)=0 .
$$

The useful identities used in the main text are

$$
\begin{aligned}
& z U_{\nu}^{\prime \prime}(z)+(1+2 \nu) U_{\nu}^{\prime}(z)+z U_{\nu}(z)=0, U_{\nu}(z) \equiv z^{-\nu} J_{\nu}(z) . \\
& \partial_{z}\left(z^{\nu} J_{\nu}(z)\right)=z^{\nu} J_{\nu-1}(z) . \\
& \partial_{z}\left(z^{-\nu} J_{\nu}(z)\right)=-z^{-\nu} J_{\nu+1}(z) . \\
& \int_{0}^{\frac{\pi}{2}} d \theta J_{p-q}\left(r_{1} \cos \theta\right) J_{q-1}\left(r_{2} \sin \theta\right)(\cos \theta)^{p-q+1}(\sin \theta)^{q}=r_{1}^{p-q} r_{2}^{q-1}\left(r_{1}^{2}+r_{2}^{2}\right)^{-\frac{p}{2}} J_{p}\left(\sqrt{r_{1}^{2}+r_{2}^{2}}\right) \\
& p, q=0, \frac{1}{2}, 1, \frac{3}{2}, 2, \ldots \\
& r^{-\frac{n-2}{2}} J_{\frac{n-2}{2}}(r)=2^{\frac{n-2}{2}} \Gamma\left(\frac{n}{2}-1\right) \sum_{k=0}^{\infty}(-)^{k}\left(k+\frac{n}{2}-1\right) r_{1}^{\frac{n-2}{2}} r_{2}^{\frac{n-2}{2}} J_{\frac{n-2}{2}}\left(r_{1}\right) J_{\frac{n-2}{2}}\left(r_{2}\right) \mathcal{C}_{k}^{\frac{n-2}{2}}(\cos \varphi) \\
& r^{2}=r_{1}^{2}+r_{2}^{2}+2 r_{1} r_{2} \cos \varphi
\end{aligned}
$$

where $n-2=0,1,2, \ldots$ and $\mathcal{C}_{m}^{p}(t)$ are Gegenbauer polynomials

$$
\begin{gathered}
\mathcal{C}_{m}^{p}(t)=\frac{2^{m} \Gamma(p+m)}{m ! \Gamma(p)}\left[t^{m}-\frac{m(m-1}{2^{2}(p+m-1)} t^{m-2}+\frac{m(m-1)(m-2)(m-3)}{2^{4} \times 1 \times 2(p+m-1)(p+m-2)} t^{m-4}+\ldots\right] . \\
J_{-\frac{1}{2}}(z)=\sqrt{\frac{2}{\pi z}} \cos z \\
\Gamma\left(\frac{n-2}{2}\right) \sum_{m=0}^{\infty} i^{m}\left(m+\frac{n-2}{2}\right) \frac{J_{m+\frac{n-2}{2}}(t)}{\left(\frac{t}{2}\right)^{\frac{n-2}{2}}} \mathcal{C}_{m}^{\frac{n-2}{2}}(x)=e^{i t x} .
\end{gathered}
$$

\section{F Tensor fields of Weyl invariant dilaton gravity}

The transformation laws (248) mix dilaton with metric and therefore the dilaton $D(x)$ is not a conventional scalar field $D^{\prime}(x)$ which should transform under the Weyl dilations and $x$-diffeomorphisms as follows

$$
\delta D^{\prime}(x)=\left(-\xi^{m} \partial_{m}+2 \alpha\right) D^{\prime}(x)
$$

As a consequence, the "quantized volume" action (245) being expressed in terms of the original fields which include dilaton $D$, though being by construction reparametrization and Weyl-invariant, does not have the conventional form of gravity + matter systems. 
However, there exists the redefinition (250) after which the action takes the conventional form. Here we prove the last statement.

Consider the "quantized volume" action (231) in the quadratic ansatz case (234). It is expanded in semiclassical power series (232), written schematically as

$$
\mathcal{A}[H]=\mathcal{A}_{0}[H]+\hbar^{2} \mathcal{A}_{2}[H]+o\left(\hbar^{4}\right)
$$

By the very construction, it is invariant w.r.t. similarity transformations (247) which are expanded in power series in $\hbar$,

$$
\delta H_{2}(x, p)=\delta_{0} H_{2}+\hbar^{2} \delta_{2} H_{2}
$$

Comparing two last formulae one finds

$$
\begin{gathered}
\delta_{0} \mathcal{A}_{0}=0 \\
\delta_{0} \mathcal{A}_{2}+\delta_{2} \mathcal{A}_{0}=0,
\end{gathered}
$$

where $\delta_{0}, \delta_{2}$ demote the variation of the action due to $\delta_{0}, \delta_{2}$ variations in (577). The first row is just a classical invariance of the cosmological term. The second row is more interesting. It tells us that the classical, $\delta_{0}$, variation of $\mathcal{A}_{2}$ is not a boundary term. This is because $\mathcal{A}_{2}$ contains the "bad term" which does not transform like " $\sqrt{g} \times\left(\right.$ scalar of correct Weyl weight)" w.r.t. diffeomorphisms $\xi^{m}(x)$ and Weyl dilations $\alpha(x)$.

Let us consider for the moment the diffeomorphisms only. Then the dilaton's kinetic term $\sim \hbar^{2} \int d^{d} x \sqrt{g} D^{\frac{d}{2}-3} g^{m n} D_{, m} D_{, n}$ is invariant, up to higher degrees in $\hbar^{2}$. Therefore, the only contribution to $\delta_{0} \mathcal{A}_{2}$ comes from the $Y$-term which reads

$$
v_{d} \int d^{d} x \sqrt{g}\left\{-\frac{\hbar^{2} d}{8} D^{\frac{d}{2}-1} Y,\right\}
$$

( $v_{d}$ is the volume of a unit ball) where $Y$ is an expression built entirely in terms of the metric and its derivatives (246). Let us write down the variation of $Y$ w.r.t. diffeomorphisms as

$$
\delta_{0} Y=-\xi^{m} \partial_{m} Y+\tilde{\delta} Y,
$$

such that $\tilde{\delta} Y$ measures "non-scalarity" of $Y$. Then the only contribution to $\delta_{0} \mathcal{A}_{2}$ comes from $\tilde{\delta} Y$.

Despite of the non-invariance of $\mathcal{A}_{2}$ w.r.t. classical gauge transformations $\delta_{0}$ the full action (576) is invariant, that is reflected by the second row of (578). This means that the non-invariant, due to $\tilde{\delta} Y$, term in the classical variation of $\mathcal{A}_{2}$ is compensated by the contribution coming from the $\delta_{2}$-variation of the classical term $\mathcal{A}_{0}$. In fact, the only 
$\delta_{2}$ variations are due to $\hbar^{2}$-terms in the variation of $D(x)$ in (248). They cancel the $\delta_{0^{-}}$ variation of $\mathcal{A}_{2}$, i.e. they cancel (up to a boundary term) the variation of (579) due-to non-scalarity of the $\delta_{0} Y$. Writing the second row of (578) explicitly one gets

$$
\begin{gathered}
0=\int d^{d} x \sqrt{g}\left\{\frac{d}{2} D^{\frac{d}{2}-1} \delta_{2} D-\frac{d}{8} D^{\frac{d}{2}-1} \delta_{0} Y\right\}+\text { boundary terms }= \\
=\int d^{d} x \sqrt{g} \frac{d}{2} D^{\frac{d}{2}-1}\left\{\delta_{2} D-\frac{1}{4} \tilde{\delta} Y\right\}+\text { boundary terms }
\end{gathered}
$$

The expression in braces in the second row does not depend on $D(x)$, therefore it is identically zero,

$$
0=\delta_{2} D-\frac{1}{4} \tilde{\delta} Y
$$

This is the result we need. It tells us that the $\delta_{2}$ variation of the dilaton w.r.t. diffeomorphisms may be cancelled by the $\tilde{\delta}$ variation of $Y$. But as $Y$ depends on metric and its derivatives only while for metric the full variation consists of $\delta_{0}$ term, it appears that one can construct the modified scalar $\tilde{D}(x)$ which transforms as a conventional scalar field w.r.t. diffeomorphisms,

$$
\begin{gathered}
\tilde{D}(x)=D(x)-\frac{\hbar^{2}}{4} Y, \\
\delta \tilde{D}(x)=-\xi^{m} \partial_{m} \tilde{D}(x) .
\end{gathered}
$$

Indeed, the $\delta_{2}$ variation of $D(x)$ is cancelled by the $\tilde{\delta}$ variation of $Y$.

Next, by direct calculations one gets the transformations properties w.r.t. Weyl dilations,

$$
\begin{gathered}
\delta g^{m n}=2 \alpha g^{m n}, \quad \delta D=2 \alpha D+\frac{\hbar^{2}}{2} g^{m n} \alpha_{, m n}, \\
\delta Y=\frac{2}{3}(d-1) g^{m n} \alpha_{, m n}+\frac{2}{3}(d-4) \alpha_{, n}\left(\frac{1}{\sqrt{g}} \partial_{m} \sqrt{g} g^{m n}\right) \\
\delta R=2(d-1) g^{m n} \alpha_{, m n}+2(d-1) \alpha_{, n}\left(\frac{1}{\sqrt{g}} \partial_{m} \sqrt{g} g^{m n}\right) \\
\delta D-\frac{\hbar^{2}}{4}(Y+\kappa R)=2 \alpha\left(D-\frac{\hbar^{2}}{4}(Y+\kappa R)\right)+ \\
+\frac{\hbar^{2}}{2} g^{m n} \alpha_{, m n}\left(1-\frac{1}{3}(d-1)-\kappa(d-1)\right)+\left(\frac{1}{\sqrt{g}} \partial_{m} \sqrt{g} g^{m n}\right)\left(-\frac{1}{4}\right)\left(\frac{2}{3}(d-4)+2 \kappa(d-1)\right),
\end{gathered}
$$

for $R$ being the scalar curvature and $\kappa$ is an arbitrary, wherefrom it follows that if one takes

$$
\kappa=\frac{4-d}{3(d-1)}
$$

then the modified scalar

$$
D^{\prime}=D-\frac{\hbar^{2}}{4}\left(Y-\frac{d-4}{3(d-1)} R\right)
$$


transforms as it is required in (249).

\section{G Free massless scalar and conformal group.}

It is useful to describe free massless scalar, satisfying the Klein-Gordon equation

$$
\square \psi(x)\left(x^{m}\right)=0, m=0,1, \ldots d-1, x^{m} \in \mathbf{R}^{d-q, q}
$$

by lifting this system to $d+2$ dimensional pseudo-Euclidean space with signature $d-q+$ 1, $q+1$ parameterized by coordinates $Y^{A} \in \mathbf{R}^{d-q+1, q+1}, A=0, \ldots d+1$. The original $d$-dimensional space is realized as a factor space of the light cone

$$
Y^{2} \equiv Y^{A} Y_{A}=0
$$

by the equivalence relation

$$
Y^{A} \sim \lambda Y^{A}, \lambda \in \mathbf{R}_{+},
$$

The $s o(d-q+1, q+1)$ generators are

$$
M_{A B}=-i \hbar\left(Y_{A} \partial_{B}-Y_{B} \partial_{A}\right)
$$

Let $U$ be an open domain in $\mathbf{R}^{d-q+1, q+1}$ which includes the light cone. Consider the space of equivalence classes $Q_{k}$ of scalar fields on $U$ of the form

$$
\begin{gathered}
\Psi(Y) \sim \Psi(Y)+Y^{2} c(Y), \\
Y \partial_{Y} \Psi(Y)=k_{d} \Psi(Y), \quad Y \partial_{Y} c(Y)=\left(k_{d}-2\right) c(Y)
\end{gathered}
$$

where $\phi$ and $c$ are regular in $U$. Clearly, the space of equivalence classes (592) is isomorphic to the space of scalar densities on $\mathcal{M}_{d-q, q}$ of a definite conformal weight. After representing $Y^{A}, Y^{2}=0$ as $Y^{A}=v Y^{A}(x)$, where $x$ are invariants of dilations (590), the canonical projection to the space of scalar densities is given by the formula

$$
\psi(x)=\left.\Psi(Y)\right|_{v=1}
$$

Consider the equation

$$
\partial_{Y}^{2} \Psi(Y)=0
$$

Due to the identity

$$
\partial_{Y}^{2} Y^{2} c(Y)=\left(4 Y \partial_{Y}+2(d+2)+Y^{2} \partial_{Y}^{2}\right) c(Y)
$$


the equation (594) is well-defined on the in the space of equivalence classes $Q_{k}$ if

$$
k_{d}=\frac{2-d}{2}
$$

what we suppose henceforth. Therefore, the equation (594) may be expressed in terms of the scalar density $\psi(x)$. It easy to get that it is exactly the massless Klein-Gordon equation (588). Thus, one has the conformally covariant description of the free massless field in $d$ dimensions in terms of $d+2$ dimensional space of equivalence classes $Q_{\frac{2-d}{2}}$. In this realization it is easy to derive the identity the generators of the conformal group satisfy,

$$
\begin{gathered}
M_{A B} M_{C}^{B}+M_{C B} M_{A}^{B}=\hbar^{2}\left(\eta_{A C}(d-2)+Y_{A} Y_{C} \partial_{Y}^{2}+Y^{2} \partial_{A} \partial_{C}\right) \Rightarrow \\
\left(M_{A B} M^{B}{ }_{C}+M_{C B} M^{B}{ }_{A}\right) \Psi(Y) \sim \hbar^{2} \eta_{A C}(d-2) \Psi(Y) \Rightarrow \\
\left(M_{A B} M_{C}^{B}+M_{C B} M_{A}^{B}\right) \psi(x)=\hbar^{2} \eta_{A C}(d-2) \psi(x) \Rightarrow N_{A B} \psi(x)=0
\end{gathered}
$$

\section{H Coordinate representation of an operator with a given Weyl symbol}

According to the textbook formulae [51], given the operator $\hat{f}$ with the Weyl symbol $f(x, p)$, its action on the wave function $u(x)$ is represented by the integral formula

$$
(\hat{f} u)(x)=(2 \pi \hbar)^{-d} \int d^{d} y d^{d} p e^{\frac{i}{\hbar}(x-y) p} f\left(\frac{x+y}{2}\right) u(y)
$$

Let $f(x, p)$ be a power series in momenta,

$$
f(x, p)=\sum_{s=0}^{\infty} f^{m(s)}(x) p_{m_{1}} \ldots p_{m_{s}} .
$$

Let us find its action on $u(x)$. Introducing the variable $q=x-y$ and using integral representation for the $\delta$-function and its derivatives, one represents the expression (598) in the form

$$
\begin{gathered}
(\hat{f} u)(x)=\left.\sum_{s=0}^{\infty}(i \hbar)^{s} \frac{\partial}{\partial q^{m_{1}}} \ldots \frac{\partial}{\partial q^{m_{s}}}\left[f^{m(s)}\left(x-\frac{q}{2}\right) u(x-q)\right]\right|_{q=0}= \\
\sum_{s=0}^{\infty} \sum_{k=0}^{s}(-i \hbar)^{s} C_{s}^{k}\left(\frac{1}{2}\right)^{k} f_{, m(k)}^{m(k) m(s-k)}(x) u_{, m(s-k)}(x)
\end{gathered}
$$

where $C_{s}^{k}=\frac{s !}{k !(s-k) !}$ are binomial coefficients. 


\section{Weyl spinor formalism in six dimensions}

This appendix is taken from the paper [46]. Notation is as follows: capital Latin letters are used for Minkowski space indices and small Latin letters for spinor ones. The metric is chosen in the form: $\eta_{A B}=\operatorname{diag}(-,+, \ldots,+)$. The Clifford algebra of $8 \times 8$ Dirac matrices $\Gamma_{A}$ reads: $\left\{\Gamma_{A}, \Gamma_{B}\right\}=-2 \eta_{A B}$. The suitable representation for $\Gamma_{A}$ is

$$
\Gamma_{A}=\left(\begin{array}{cc}
0 & \left(\sigma_{A}\right)_{a \dot{a}} \\
\left(\widetilde{\sigma}_{A}\right)^{\dot{a} a} & 0
\end{array}\right), \quad \begin{gathered}
\sigma_{A}=\left\{1, \gamma_{0}, i \gamma_{1}, i \gamma_{2}, i \gamma_{3}, \gamma_{5}\right\} \\
\widetilde{\sigma}_{A}=\left\{1,-\gamma_{0},-i \gamma_{1},-i \gamma_{2},-i \gamma_{3},-\gamma_{5}\right\}
\end{gathered}
$$

where $\gamma_{i}, i=0,1,2,3,5$ are the ordinary Dirac matrices in four dimensions. The charge conjugation matrix is defined as

$$
C=\Gamma_{2} \Gamma_{4}=\left(\begin{array}{cc}
I & 0 \\
0 & \widetilde{I}
\end{array}\right), \quad I=\widetilde{I}=\left(\begin{array}{ccccc}
0 & 1 & & 0 \\
-1 & 0 & & \\
-- & - & - & - \\
& & 0 & 1 \\
0 & \mid & -1 & 0
\end{array}\right)
$$

The spinor representation of $S O(5,1)$ on Dirac spinors $\Psi=\left(\begin{array}{c}\lambda_{a} \\ \bar{\pi}^{\dot{b}}\end{array}\right)$ is generated by

$$
\begin{gathered}
\Sigma_{A B}=-\frac{1}{4}\left[\Gamma_{A}, \Gamma_{B}\right]=\left(\begin{array}{cc}
\left(\sigma_{A B}\right)_{a}{ }^{b} & 0 \\
0 & \left(\widetilde{\sigma}_{A B}\right)^{\dot{a}}{ }_{\dot{b}}
\end{array}\right)= \\
=\left(\begin{array}{cc}
-\frac{1}{4}\left(\sigma_{A_{a} \dot{a}} \widetilde{\sigma}_{B}{ }^{\dot{a} b}-\sigma_{B a \dot{a}} \widetilde{\sigma}_{A}{ }^{\dot{a} b}\right) & 0 \\
0 & -\frac{1}{4}\left(\widetilde{\sigma}_{A}{ }^{\dot{a} b} \sigma_{B_{b \dot{b}}}-\widetilde{\sigma}_{B}{ }^{i b} \sigma_{A_{b \dot{b}}}\right)
\end{array}\right)
\end{gathered}
$$

The representation is decomposed into two irreducible ones corresponding to the left- and right-handed Weyl spinors. It turns out that the representation (603) and its complex conjugated are equivalent: $\left(\sigma_{A B}^{*}\right)_{a}{ }^{\dot{b}}=I_{\dot{a}}{ }^{a}\left(\sigma_{A B}\right)_{a}{ }^{b} I_{b}{ }_{b}{ }^{b},\left(\widetilde{\sigma}_{A B}^{*}\right)^{a}{ }_{b}=\widetilde{I}^{a}{ }_{a}\left(\widetilde{\sigma}_{A B}\right)^{\dot{a}}{ }_{\dot{b}} \widetilde{I}^{\dot{b}}{ }_{b}$. So, one can convert the dotted spinor indices into undotted ones

$$
\bar{\lambda}_{a}=I_{a}{ }^{a} \stackrel{*}{\lambda}_{a} \quad, \quad \bar{\pi}^{a}=\widetilde{I}_{a}^{a}{ }_{a}^{*}{ }^{a}
$$

While the gradient and contragredient representations are inequivalent because of absence of an object raising and/or lowering spinor indices as distinguished from the fourdimensional case. It is convenient to turn from the matrices $\left(\sigma_{A}\right)_{a \dot{a}},\left(\widetilde{\sigma}_{A}\right)^{\dot{a} a}$ to $\left(\sigma_{A}\right)_{a b}=$ 
$\left(\sigma_{A}\right)_{a \dot{a}} \widetilde{I}_{b}^{\dot{a}},\left(\widetilde{\sigma}_{A}\right)^{a b}=\widetilde{I}_{\dot{a}}^{a}\left(\widetilde{\sigma}_{A}\right)^{\dot{a} a}$. They possess a number of relations

$$
\begin{gathered}
\left(\sigma_{A}\right)_{a b}=-\left(\sigma_{A}\right)_{b a} \quad\left(\widetilde{\sigma}_{A}\right)^{a b}=-\left(\widetilde{\sigma}_{A}\right)^{b a} \\
\left(\sigma_{A}\right)_{a b}\left(\sigma^{A}\right)_{c d}=-2 \epsilon_{a b c d} \quad\left(\widetilde{\sigma}_{A}\right)^{a b}\left(\widetilde{\sigma}^{A}\right)^{c d}=-2 \epsilon^{a b c d} \\
\left(\sigma_{A}\right)_{a b}=-\frac{1}{2} \epsilon_{a b c d}\left(\widetilde{\sigma}_{A}\right)^{c d} \quad\left(\widetilde{\sigma}_{A}\right)^{a b}=-\frac{1}{2} \epsilon^{a b c d}\left(\sigma^{A}\right)_{c d} \\
\left(\sigma_{A}\right)_{a b}\left(\widetilde{\sigma}^{A}\right)^{c d}=2\left(\delta_{a}{ }^{c} \delta_{b}{ }^{d}-\delta_{a}{ }^{d} \delta_{b}{ }^{c}\right), \quad\left(\sigma_{A}\right)_{a b}\left(\widetilde{\sigma}_{B}\right)^{b a}=-4 \eta_{A B} \\
\left(\sigma_{A}\right)_{a b}\left(\widetilde{\sigma}_{B}\right)^{b c}+\left(\sigma_{B}\right)_{a b}\left(\widetilde{\sigma}_{A}\right)^{b c}=-2 \eta_{A B} \delta_{a}{ }^{c} \\
\left(\widetilde{\sigma}_{A}\right)^{a b}\left(\sigma_{B}\right)_{b c}+\left(\widetilde{\sigma}_{B}\right)^{a b}\left(\sigma_{A}\right)_{b c}=-2 \eta_{A B} \delta^{a}{ }_{c}
\end{gathered}
$$

Here we introduced two invariant tensors $\epsilon_{a b c d}$ and $\epsilon^{a b c d}$, totally antisymmetric in indices and $\epsilon_{1234}=\epsilon^{1234}=1$. With the aid of introduced objects one may convert the vector indices into antisymmetric pairs of spinor ones. E.g. for a given vector $p_{A}$

$$
p_{A} \rightarrow p_{a b}=p_{A}\left(\sigma^{A}\right)_{a b}, \quad p^{a b}=p_{A}\left(\widetilde{\sigma}^{A}\right)^{a b}, \quad p_{A}=-\frac{1}{4} p_{a b}\left(\widetilde{\sigma}_{A}\right)^{b a}=-\frac{1}{4} p^{a b}\left(\sigma_{A}\right)_{b a}
$$

\section{References}

[1] E. S. Fradkin and A. A. Tseytlin, "Conformal Supergravity," Phys. Rept. 119, 233 (1985).

[2] J. Fang and C. Fronsdal, "Massless, Half Integer Spin Fields In De Sitter Space," Phys. Rev. D 22, 1361 (1980). C. Fronsdal, "Singletons And Massless, Integral Spin Fields On De Sitter Space (Elementary Particles In A Curved Space Vii)," Phys. Rev. D 20, 848 (1979). J. Fang and C. Fronsdal, "Massless Fields With Half Integral Spin," Phys. Rev. D 18, 3630 (1978). C. Fronsdal, "Massless Fields With Integer Spin," Phys. Rev. D 18, 3624 (1978).

[3] E. S. Fradkin and M. A. Vasiliev, "Superalgebra Of Higher Spins And Auxiliary Fields," Int. J. Mod. Phys. A 3, 2983 (1988). M. A. Vasiliev and E. S. Fradkin, "Gravitational Interaction Of Massless High Spin ( $>$ 2) Fields," JETP Lett. 44, 622 (1986) [Pisma Zh. Eksp. Teor. Fiz. 44, 484 (1986)].

[4] E. S. Fradkin and V. Y. Linetsky, "Three Phases Of A Unified Theory: String Theory, Higher Spin Gauge Theory In The Anti-De Sitter Universe, Conformal Higher Spin 
Theory?," In *Capri 1990, Proceedings, General relativity and gravitational physics* 195-245.

[5] C. Aragone and S. Deser, "Consistency Problems Of Hypergravity," Phys. Lett. B 86, 161 (1979). F. A. Berends, J. W. van Holten, B. de Wit and P. van Nieuwenhuizen, "On Spin 5/2 Gauge Fields," in C79-02-25.5 J. Phys. A 13, 1643 (1980).

[6] J. Maldacena, "The large-N limit of superconformal field theories and supergravity," Adv. Theor. Math. Phys. 2, 231 (1998), arXiv:hep-th/9711200]. S.S. Gubser, I.R. Klebanov and A.M. Polyakov, "Gauge theory correlators from non-critical string theory," Phys. Lett. B428, 105 (1998), arXiv:hep-th/9802109. E. Witten, "Anti-de Sitter space and holography," Adv. Theor. Math. Phys. 2, 253 (1998), arXiv:hepth/9802150].

[7] H. Liu and A. A. Tseytlin, " $\mathrm{D}=4$ super Yang-Mills, $\mathrm{D}=5$ gauged supergravity, and D = 4 conformal supergravity," Nucl. Phys. B 533, 88 (1998) arXiv:hep-th/9804083].

[8] B. Sundborg, "Stringy gravity, interacting tensionless strings and massless higher spins," Nucl. Phys. Proc. Suppl. 102, 113 (2001) arXiv:hep-th/0103247]. P. HaggiMani and B. Sundborg, "Free large N supersymmetric Yang-Mills theory as a string theory," JHEP 0004, 031 (2000) arXiv:hep-th/0002189.

[9] E. Witten, talk at J.H. Schwarz 60-th Birthday Conference, CalTech, November 2-3, 2001. WWW link: http://quark.caltech.edu/jhs60/witten/1-28.htm

[10] R. R. Metsaev, "Massless arbitrary spin fields in AdS(5)," Phys. Lett. B 531, 152 (2002) arXiv:hep-th/0201226]. R. R. Metsaev, "Light cone form of field dynamics in anti-de Sitter spacetime and AdS/CFT correspondence," Nucl. Phys. B 563, 295 (1999) arXiv:hep-th/9906217.

[11] A. A. Tseytlin, "On limits of superstring in $\operatorname{AdS}(5)$ x $S^{* * 5}$," $\operatorname{arXiv}: h e p-t h / 0201112$.

[12] S.E. Konstein, M.A. Vasiliev and V.N. Zaikin, "Conformal higher spin currents in any dimension and AdS/CFT correspondence," JHEP 0012, 018 (2000) arXiv:hepth/0010239].

[13] C. M. Hull, "W geometry," Commun. Math. Phys. 156, 245 (1993) arXiv:hepth/9211113].

[14] E. S. Fradkin and V. Y. Linetsky, "Cubic Interaction In Conformal Theory Of Integer Higher Spin Fields In Four-Dimensional Space-Time," Phys. Lett. B 231, 97 (1989). 
E. S. Fradkin and V. Y. Linetsky, "Superconformal Higher Spin Theory In The Cubic Approximation," Nucl. Phys. B 350, 274 (1991). E. S. Fradkin and V. Y. Linetsky, "A Superconformal Theory Of Massless Higher Spin Fields In D = $(2+1)$," Mod. Phys. Lett. A 4, 731 (1989) [Annals Phys. 198, 293 (1990)].

[15] C. Aragone, S. Deser and Z. Yang, "Massive Higher Spin From Dimensional Reduction Of Gauge Fields," Annals Phys. 179, 76 (1987).

[16] V. E. Lopatin and M. A. Vasiliev, "Free Massless Bosonic Fields Of Arbitrary Spin In D-Dimensional De Sitter Space,” Mod. Phys. Lett. A 3, 257 (1988).

[17] R. R. Metsaev, "Arbitrary spin massless bosonic fields in d-dimensional anti-de Sitter space," arXiv:hep-th/9810231. R. R. Metsaev, "Lowest Eigenvalues Of The Energy Operator For Totally (Anti)Symmetric Massless Fields Of The N-Dimensional AntiDe Sitter Group," Class. Quant. Grav. 11, L141 (1994).

[18] I. L. Buchbinder, A. Pashnev and M. Tsulaia, "Massless higher spin fields in the AdS background and BRST: Constructions for nonlinear algebras," arXiv:hepth/0206026. I. L. Buchbinder, A. Pashnev and M. Tsulaia, "Lagrangian formulation of the massless higher integer spin fields in the AdS background," Phys. Lett. B 523, 338 (2001) arXiv:hep-th/0109067.

[19] T. Biswas and W. Siegel, "Radial dimensional reduction: (Anti) de Sitter theories from flat," JHEP 0207, 005 (2002) arXiv:hep-th/0203115.

[20] M. A. Vasiliev, "Consistent Equation For Interacting Gauge Fields Of All Spins In (3+1)-Dimensions," Phys. Lett. B 243, 378 (1990). M. A. Vasiliev, "Algebraic Aspects Of The Higher Spin Problem," Phys. Lett. B 257, 111 (1991).

[21] M. A. Vasiliev, "Progress in higher spin gauge theories," arXiv:hep-th/0104246. M. A. Vasiliev, "Higher spin symmetries, star-product and relativistic equations in AdS space," arXiv:hep-th/0002183.

[22] K. B. Alkalaev and M. A. Vasiliev, " $\mathrm{N}=1$ supersymmetric theory of higher spin gauge fields in $\operatorname{AdS}(5)$ at the cubic level," arXiv:hep-th/0206068.

[23] M. A. Vasiliev, "Cubic interactions of bosonic higher spin gauge fields in AdS(5)," Nucl. Phys. B 616, 106 (2001) arXiv:hep-th/0106200.

[24] R. R. Metsaev, "Cubic interaction vertices for higher spin fields," arXiv:hepth/9705048. 
[25] J. Engquist, E. Sezgin and P. Sundell, "On N=1,2,4 Higher Spin Gauge Theories in Four Dimensions," arXiv:hep-th/0207101. E. Sezgin and P. Sundell, "Massless higher spins and holography," arXiv:hep-th/0205131.

[26] B. de Wit and D. Z. Freedman, "Systematics Of Higher Spin Gauge Fields," Phys. Rev. D 21, 358 (1980).

[27] P. Claus, R. Kallosh, J. Kumar, P. K. Townsend and A. Van Proeyen, "Conformal theory of M2, D3, M5 and D1+D5 branes," JHEP 9806, 004 (1998) arXiv:hepth/9801206].

[28] E. Bergshoeff, M. J. Duff, C. N. Pope and E. Sezgin, "Supersymmetric Supermembrane Vacua And Singletons," Phys. Lett. B 199, 69 (1987). E. Bergshoeff, A. Salam, E. Sezgin and Y. Tanii, "Singletons, Higher Spin Massless States And The Supermembrane," Phys. Lett. B 205, 237 (1988).

[29] M. J. Duff, "Twenty years of the Weyl anomaly," Class. Quant. Grav. 11, 1387 (1994) arXiv:hep-th/9308075]. S. Deser, "Closed form effective conformal anomaly actions in D $\geq$ 4," Phys. Lett. B 479, 315 (2000) arXiv:hep-th/9911129]. S. Deser and A. Schwimmer, "Geometric Classification Of Conformal Anomalies In Arbitrary Dimensions," Phys. Lett. B 309, 279 (1993) arXiv:hep-th/9302047.

[30] M. Henningson and K. Skenderis, "The holographic Weyl anomaly," JHEP 9807, 023 (1998) arXiv:hep-th/9806087. S. de Haro, S. N. Solodukhin and K. Skenderis, "Holographic reconstruction of spacetime and renormalization in the AdS/CFT correspondence," Commun. Math. Phys. 217, 595 (2001) arXiv:hep-th/0002230.

[31] A. O. Barvinsky and G. A. Vilkovisky, "The Generalized Schwinger-Dewitt Technique In Gauge Theories And Quantum Gravity," Phys. Rept. 119, 1 (1985).

[32] E. Sezgin and P. Sundell, "7D bosonic higher spin theory: Symmetry algebra and linearized constraints," arXiv:hep-th/0112100.

[33] I. Bars, "2T physics 2001," arXiv:hep-th/0106021. I. Bars, "U*(1,1) noncommutative gauge theory as the foundation of 2T-physics in field theory," Phys. Rev. D 64, 126001 (2001) arXiv:hep-th/0106013. I. Bars and C. Deliduman, "High spin gauge fields and two-time physics," Phys. Rev. D 64, 045004 (2001) arXiv:hep-th/0103042. I. Bars and S. J. Rey, "Noncommutative $\operatorname{Sp}(2, \mathrm{R})$ gauge theories: A field theory approach to two-time physics," Phys. Rev. D 64, 046005 (2001) arXiv:hep-th/0104135. 
[34] A. Ferber, "Supertwistors And Conformal Supersymmetry," Nucl. Phys. B 132 (1978) 55. T. Shirafuji, "Lagrangian Mechanics Of Massless Particles With Spin," Prog. Theor. Phys. 70, 18 (1983). A. K. Bengtsson, I. Bengtsson, M. Cederwall and N. Linden, "Particles, Superparticles And Twistors," Phys. Rev. D 36 (1987) 1766. D. P. Sorokin, V. I. Tkach and D. V. Volkov, "Superparticles, Twistors And Siegel Symmetry," Mod. Phys. Lett. A 4 (1989) 901.

[35] I. Bars, "2T physics formulation of superconformal dynamics relating to twistors and supertwistors," Phys. Lett. B 483, 248 (2000) [arXiv:hep-th/0004090].

[36] M. Cederwall, "Geometric construction of AdS twistors," Phys. Lett. B 483, 257 (2000) arXiv:hep-th/0002216]. I. Bengtsson and M. Cederwall, "Particles, Twistors And The Division Algebras," Nucl. Phys. B 302, 81 (1988).

[37] D. P. Sorokin, V. I. Tkach, D. V. Volkov and A. A. Zheltukhin, "From The Superparticle Siegel Symmetry To The Spinning Particle Proper Time Supersymmetry," Phys. Lett. B 216 (1989) 302.

[38] E. Sezgin and P. Sundell, "Towards massless higher spin extension of D = 5, N = 8 gauged supergravity," JHEP 0109, 025 (2001) arXiv:hep-th/0107186. "Doubletons and 5D higher spin gauge theory," JHEP 0109, 036 (2001) arXiv:hep-th/0105001.

[39] O. V. Shaynkman and M. A. Vasiliev, "Higher spin conformal symmetry for matter fields in 2+1 dimensions," Theor. Math. Phys. 128, 1155 (2001) [Teor. Mat. Fiz. 128, 378 (2001)] arXiv:hep-th/0103208].

[40] P. Bozhilov, "Null branes in string theory backgrounds," Phys. Rev. D 62, 105001 (2000) arXiv:hep-th/9911210.

[41] M. Eastwood, "Higher symmetries of the Laplacian," arXiv:hep-th/0206233.

[42] L. Brink and J. H. Schwarz, "Quantum Superspace," Phys. Lett. B 100, 310 (1981).

[43] E. Witten, "Twistor - Like Transform In Ten-Dimensions," Nucl. Phys. B 266, 245 (1986).

[44] M. S. Plyushchay, "Massless Point Particle With Rigidity," Mod. Phys. Lett. A 4, 837 (1989). A. Nersessian, "Massless particles and the geometry of curves: Classical picture," arXiv:hep-th/9911020.

[45] S. K. Wong, "Field And Particle Equations For The Classical Yang-Mills Field And Particles With Isotopic Spin," Nuovo Cim. A 65, 689 (1970). 
[46] S. L. Lyakhovich, A. A. Sharapov and K. M. Shekhter, "Spinning particle dynamics on six-dimensional Minkowski space," J. Math. Phys. 38, 4086 (1997) arXiv:hepth/9609133.

[47] A. Schwarz, "Superanalogs Of Symplectic And Contact Geometry And Their Applications To Quantum Field Theory," arXiv:hep-th/9406120.

[48] F. Bayen, M. Flato, C. Fronsdal, A. Lichnerowicz and D. Sternheimer, "Deformation Theory And Quantization. 2. Physical Applications," Annals Phys. 111, 111 (1978). F. Bayen, M. Flato, C. Fronsdal, A. Lichnerowicz and D. Sternheimer, "Deformation Theory And Quantization. 1. Deformations Of Symplectic Structures," Annals Phys. 111, 61 (1978).

[49] J. Bagger, J. Wess, Supersymmetry and Supergravity, Princeton Univ. Press, 1992.

[50] V.I. Arnold, Mathematical methods of classical mechanics, Springer-Verlag, New York, 1989.

[51] F.A.Berezin, The Method of Second Quantization, Academic Press, New York 1966. F. Berezin, M. Shubin, The Schr”odinger equation, Kluwer, 1991.

[52] N. Ja. Vilenkin, Special Functions and the Theory of Group Representations, American Mathematical Society, Providence, R. I., 1968.

[53] N.D. Birrell and P.C.W. Davies, Quantum Fields in Curved Space, Cambridge University Press, New York 1982.

[54] A. Y. Segal, in preparation.

[55] A. Y. Segal, work in progress.

[56] A. Y. Segal, "Point particle in general background fields vs. free gauge theories of traceless symmetric tensors," arXiv:hep-th/0110056.

[57] A. Y. Segal, "A generating formulation for free higher spin massless fields," arXiv:hep-th/0103028.

[58] A. Y. Segal, "Point particle in general background fields and generalized equivalence principle," arXiv:hep-th/0008105. 